\title{
THEORETICAL AND COMPUTATIONAL MODELING OF NATURALLY AND ARTIFICIALLY MODIFIED RNA NUCLEOTIDES
}

\author{
A Dissertation presented to \\ the Faculty of the Graduate School \\ at the University of Missouri
}

In Partial Fulfillment

of the Requirements for the Degree

Doctor of Philosophy

by

TRAVIS CALEB HURST

Dr. Shi-Jie Chen, Dissertation Supervisor

MAY 2021 
The undersigned, appointed by the Dean of the Graduate School, have examined the dissertation entitled:

\section{THEORETICAL AND COMPUTATIONAL MODELING OF NATURALLY AND ARTIFICIALLY MODIFIED RNA NUCLEOTIDES}

presented by Travis Caleb Hurst, a candidate for the degree of Doctor of Philosophy and hereby certify that, in their opinion, it is worthy of acceptance.

Dr. Shi-Jie Chen

Dr. Xiao Heng

Dr. Gavin M. King

Dr. Ioan Kosztin

Dr. Xiaoqin Zou 


\section{DEDICATION}

The glory of my work goes to Jesus Christ, who strengthens and enlightens me.

I dedicate this dissertation to my grandfathers, Don Crader and Carl Hurst. They left legacies of exploration, intellectual pursuit, and tenacity. Their leadership and advice is sorely missed.

I am thankful for my stunning wife, Alyson, who supports and sacrifices on my behalf. Thank you for your patience and grace. I would also like to thank my parents-Clay and Becky Hurst — for their time, advice, energy, and support during my pursuit of this degree. I have benefited from your examples of perseverance and wisdom all my life.

A few great teachers along the way can make all of the difference. I would be remiss not to thank those who made a difference for me. Thank you Jean Gibbs, Carol Keen, and Christy Shinn. Thank you Drs. William Banks, Shi-Jie Chen, John Fresen, Gavin King, Sergei Kopeikin, Dorina Kosztin, Ioan Kosztin, John McCormick, and Dan Peterson. Your inspiration, encouragement, and knowledge are appreciated.

I would also like to thank many peers and friends for their camaraderie and encouragement, including Seth Allen, Matt Anderson, Dr. Andy Basnett, Dr. Joe Basnett, Zak Beach, Danielle Woodfin, Mackenzie Sprandel, Josh Bradley, Matt Crader, Zack DuPerier, Brock Evans, Sean Fayfar, Caleb Harris, Caleb Hosey, Taylor Houston, Dr. Brian Jimenez, James Jones, Alex Kiehl, Dylan Macoubrie, Dr. Eddie Pluhar, Brett Renfroe, Dr. Milica Utjesanovic, Ty Williams, and Dr. Jon Young. To you and others not listed here, thank you for your support, discussions, and companionship along the way. 


\section{ACKNOWLEDGMENTS}

My exploration of biological physics has been enriched by a host of supportive individuals. Here, I would like to thank those who aided in my development and made my research possible.

I gratefully acknowledge the funding received toward my $\mathrm{PhD}$ from the American taxpayer through the National Science Foundation Graduate Research Fellowship and grants won by Dr. Shi-Jie Chen from the National Institutes of Health.

Thank you to Dr. Shi-Jie Chen for your inspirational leadership, devoted determination, and continuous support. Always encouraging, motivational, and full of ideas, he sets a high standard for himself in all aspects, which elevates the work of those around him. Personally, I have been galvanized by his broad vision, detailed insight, guidance, and generosity. I am honored to have him as my advisor.

Thank you to my other committee members, Drs. Xiao Heng, Gavin King, Ioan Kosztin, Xiaoqin Zou. They committed considerable time and effort to helping me, greatly bolstering my professional development through rigorous courses, discussions, and guidance.

Thank you to all of the members of Dr. Chen's group, whom I had the good fortune to work with. Thanks to the current lab members-Sicheng Zhang, Yuanzhe Zhou, and Drs. Yangwei Jiang, Lei Jin, and Jun Li, —and thanks to the former lab members, Drs. Chenhan Zhao, Xiaojun Xu, Peinan Zhao, Lizhen Sun, Yi Cheng, and Dong Zhang. Your time, collaboration, and support are valued and appreciated. 


\section{TABLE OF CONTENTS}

ACKNOWLEDGMENTS $\ldots \ldots \ldots \ldots \ldots \ldots \ldots \ldots$ ii

LIST OF TABLES $\ldots \ldots \ldots \ldots \ldots \ldots \ldots \ldots \ldots \ldots$ ix

LIST OF FIGURES $\ldots \ldots \ldots \ldots \ldots \ldots \ldots \ldots \ldots \ldots \ldots \ldots \ldots$ xii

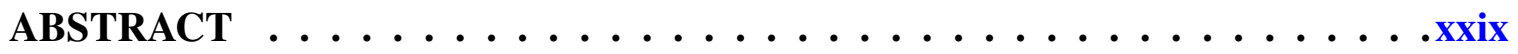

\section{CHAPTER}

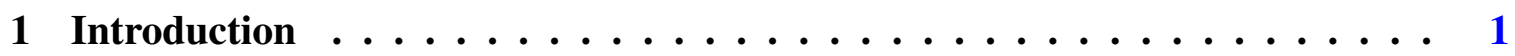

1.1 Reviewing RNA fundamentals $\ldots \ldots \ldots \ldots \ldots \ldots$

1.2 Examining significance of SHAPE probes $\ldots \ldots \ldots \ldots \ldots$

1.3 Using coarse-grained simulations to fold RNA . . . . . . . . . . . 6

1.4 Assessing significance of modified nucleotides . . . . . . . . . . 7

1.5 Briefly summarizing the main results $\ldots \ldots \ldots \ldots \ldots \ldots$

1.5.1 Project 1: Predicting SHAPE reactivity from 3D structure information 11

1.5.2 Project 2: Comparing analytical modeling to deep learning approaches for estimating SHAPE reactivity . . . . . . . . . . 12

1.5.3 Project 3: Evaluating mechanisms driving reactivity bias and sieving RNA structures with SHAPE . . . . . . . . . . . . . 12

1.5.4 Project 4: Building an efficient coarse-grained folding force field . . 13

1.5.5 Project 5: Deciphering nucleotide modification-induced structure and stability changes . . . . . . . . . . . . . . . . 14

2 Quantitative Understanding of SHAPE Mechanism from RNA Structure and Dynamics Analysis $\ldots \ldots \ldots \ldots \ldots \ldots \ldots$ 


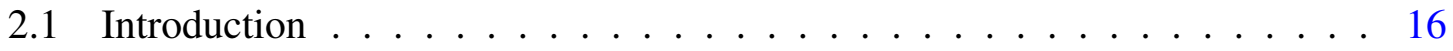

2.2 Methods . . . . . . . . . . . . . . . . . . . . 20

2.2.1 Interaction intensity for base pairs and base stacks . . . . . . . 20

2.2.2 Ligand accessibility calculation $\ldots \ldots \ldots \ldots \ldots . \ldots 22$

2.2.3 All-atom molecular dynamics sampling of near-native structure . . . 22

2.3 Results . . . . . . . . . . . . . . . . . . . . . . . . 27

2.3.1 The three-Dimensional Structure-SHAPE Relationship (3DSSR) function: Prediction of SHAPE profiles from 3D structure and energetics 32

2.4 Discussion . . . . . . . . . . . . . . . . . . . 38

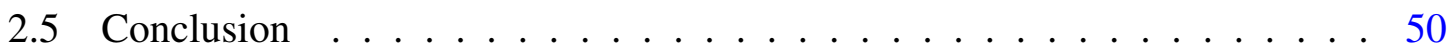

3 Analytical modeling and deep learning approaches to estimating RNA SHAPE reactivity from 3D structure $\ldots \ldots \ldots \ldots \ldots \ldots \ldots \ldots$

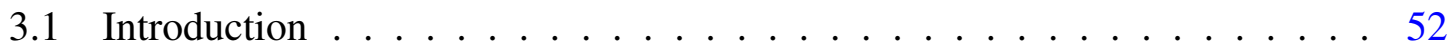

3.2 Methods . . . . . . . . . . . . . . . . . . . 56

3.2.1 Finding structures corresponding to SHAPE data $\ldots \ldots \ldots \ldots 6$

3.2.2 Reviewing 3DSSR methods and conclusions . . . . . . . . . 56

3.2.3 Applying the model to the SAM-I/IV riboswitch aptamer . . . . . 59

3.2.4 Using a CNN to predict SHAPE reactivity from structure . . . . . 61

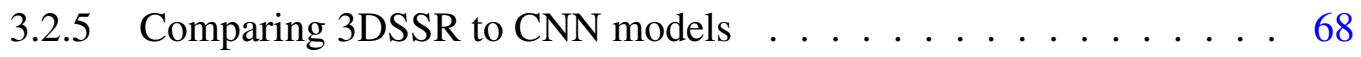

3.3 Conclusion . . . . . . . . . . . . . . . . . . . . 69

4 Sieving RNA 3D Structures with SHAPE and Evaluating Mechanisms Driving Sequence-Dependent Reactivity Bias . . . . . . . . . 71

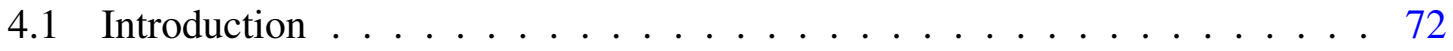


4.2 Theory and Methods $\ldots \ldots \ldots \ldots \ldots \ldots \ldots$

4.2.1 Base pairing and stacking interactions $\ldots \ldots \ldots 75$

$4.2 .2 \quad 2 \mathrm{D}$ structure $\ldots \ldots \ldots \ldots \ldots \ldots$

4.2.3 Accounting for Other Structural Features _. . . . . . . . . 79

4.2 .4 Predicting SHAPE $\ldots \ldots \ldots \ldots$

4.2.5 All-atom simulations to form near-native ensembles . . . . . . . 82

4.2.6 Generating non-native decoy structures with coarse grained simulations . . . . . . . . . . . . . . . 83

$4.2 .7 \quad$ Log-normal noise . . . . . . . . . . . . . . . . . . . 84

4.2.8 Training the model with simulated annealing $\ldots \ldots$. . . . . 87

4.2.9 Sequence-dependent bias in SHAPE experiments . . . . . . . . 89

4.2.10 REUS to find mechanism for sequence-dependent bias . . . . . . 91

4.3 Results . . . . . . . . . . . . . . . . . . . . . . . 994

4.3.1 Enhanced performance . . . . . . . . . . . . . . 94

4.3.2 Identifying tertiary contacts with SHAPE $\ldots \ldots \ldots$. . . . 96

4.3.3 The $1 \mathrm{M} 7$ stability in the binding pocket largely explains bias . . . 97

4.4 Discussion . . . . . . . . . . . . . . . . . . . . . . 99

4.4.1 Improved performance $\ldots \ldots \ldots \ldots 9 . \ldots \ldots$

4.4.2 Determining sequence-dependent reactivity bias mechanism . . 100

4.4.3 Detailing experimental artifacts hampering performance $\ldots \ldots 102$

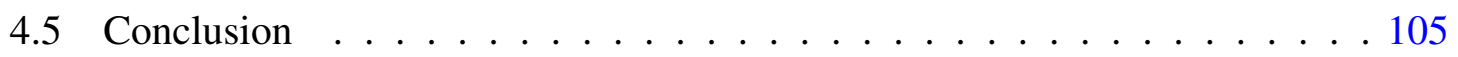

5 A Bayes-inspired theory for optimally building an efficient coarse-grained folding force field . . . . . . . . . . . . . . . 107 


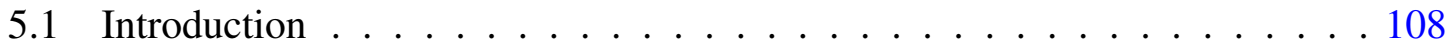

5.2 Theory and Methods $\ldots \ldots \ldots \ldots \ldots \ldots \ldots \ldots \ldots$

5.2 .1 Setting up the system . . . . . . . . . . . . . . 112

5.2.2 Supporting the IsRNA theory with statistical mechanics . . . . . . 113

5.2.3 Generalizing and numerically stabilizing the model $\ldots \ldots \ldots$. . 115

5.2.4 Maximizing entropy to optimize model construction $\ldots \ldots \ldots 121$

5.2.5 Applying the model to fold RNA . . . . . . . . . . . . . . 124

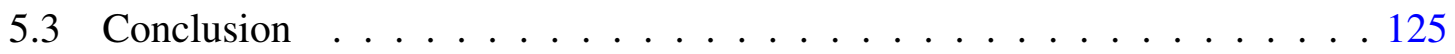

6 Deciphering nucleotide modification-induced structure and stability changes 128

6.1 Introduction . . . . . . . . . . . . . . . . . . . . . . 129

6.2 Materials and Methods . . . . . . . . . . . . . . . . . . . 134

6.2.1 Selecting and building systems $\ldots \ldots \ldots \ldots \ldots \ldots$

6.2.2 Using alchemical simulation results to estimate free energy changes 136

6.2.3 Analyzing TREMD trajectories . . . . . . . . . . . . . . . 137

6.3 Results and Discussion . . . . . . . . . . . . . . . . . . . 139

6.3.1 Comparing duplex simulation results to thermal denaturation data . 140

6.3.2 Determining mutation/modification-induced structural mechanisms

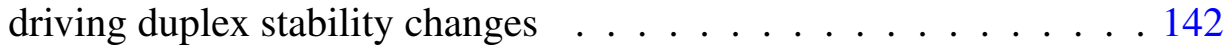

6.3.3 Comparing hairpin PMFs to thermal denaturation data $\ldots . . .144$

6.3.4 Investigating modification-induced hairpin structural alterations $\ldots 147$

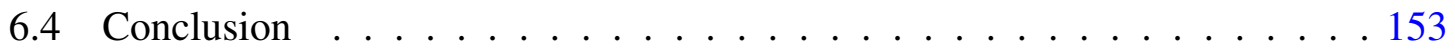

\section{APPENDIX}




\section{A Appendix to Quantitative Understanding of SHAPE Mechanism from RNA}

Structure and Dynamics Analysis $\ldots \ldots \ldots \ldots \ldots \ldots$

A.1 Visualizing the bound SHAPE ligand $\ldots \ldots \ldots \ldots \ldots \ldots$

A.2 Leave-one-out parameter optimization $\ldots \ldots \ldots \ldots \ldots$

A.3 Experimental conditions of SHAPE experiments . . . . . . . . . 157

A.4 Tabulating native and decoy 2D structures $\ldots \ldots \ldots \ldots \ldots$

A.5 Evaluating performance of SHAPE predictions $\ldots \ldots \ldots \ldots$

B Appendix to Sieving RNA 3D Structures with SHAPE and Evaluating Mechanisms Driving Sequence-Dependent Reactivity Bias . . . . . . . . . . 169

B.1 Base pairing frequency table $\ldots \ldots \ldots \ldots \ldots \ldots$

B.2 Training parameters and $2 \mathrm{D}$ structures $\ldots \ldots \ldots \ldots \ldots \ldots \ldots$

B.3 Umbrella sampling to determine unreacted 1M7 stability in the reactive site 183

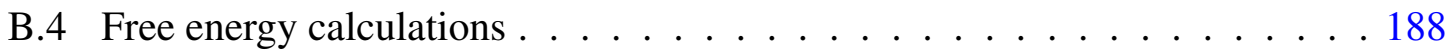

C Appendix to Deciphering nucleotide modification-induced structure and stability changes . . . . . . . . . . . . . . . . . . 193

C.1 Thermal denaturation data for RNA duplexes $\ldots \ldots \ldots \ldots$

C.2 Running and quantitatively validating alchemical simulations on duplex system . . . . . . . . . . . . . . . . . . . . 195

C.2.1 Setting up and running simulations . . . . . . . . . . . 195

C.2.2 Validating duplex topology comparisons . . . . . . . . . . 197

C.3 Enhancing hairpin sampling with TREMD . . . . . . . . . . . . 198

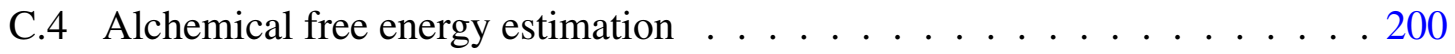

C.5 Estimated free energies from individual alchemical runs . . . . . . . . . 202

C.6 Predicted melting curve . . . . . . . . . . . . . . . . . . 203 
C.7 Comparing estimators of relative free energy change . . . . . . . . . 204

C.8 Calculating twice-perturbed free energies, propagating uncertainty, and evaluating computational efficiency . . . . . . . . . . . . . 206

C.9 Confirming convergence of hairpin TREMD PMF . . . . . . . . . 210

C.10 Calculating free energy changes from hairpin PMFs $\ldots \ldots \ldots \ldots . \ldots 211$

C.11 Evaluating convergence and phase space overlap in alchemical simulations . 213

C.12 Analyzing mechanisms causing free energy changes . . . . . . . 215

C.13 A "spring-loaded" modification . . . . . . . . . . . . . . . . . . 216

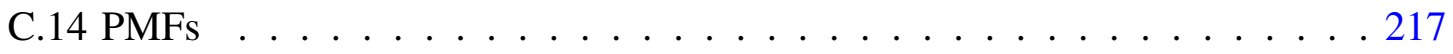

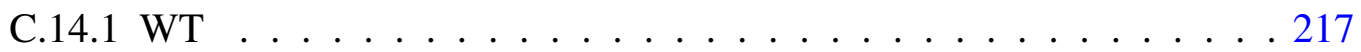

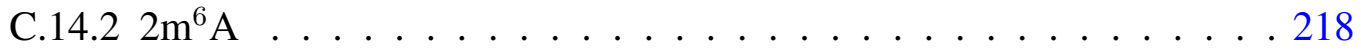

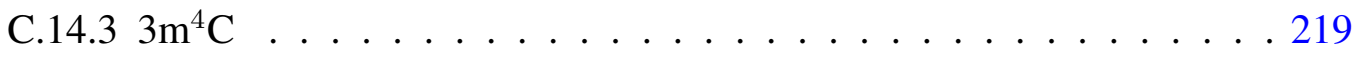

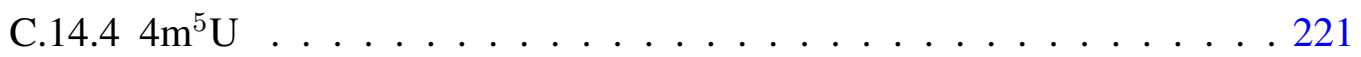

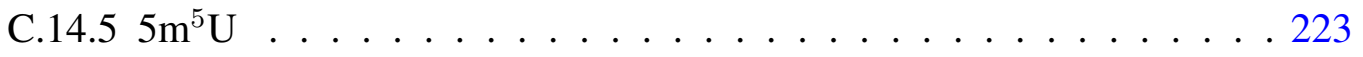

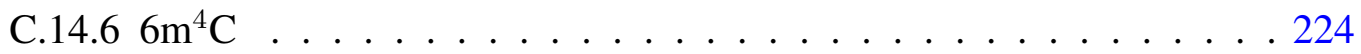

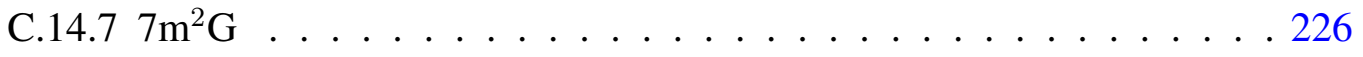

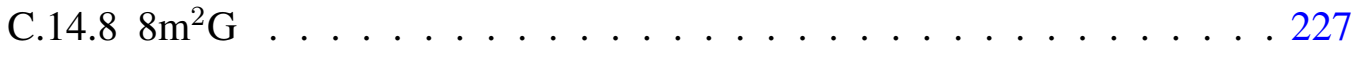

C.14.9 $9 \mathrm{~m}^{5} \mathrm{U} \ldots \ldots \ldots \ldots \ldots$. . . . . . . . . . . . . . . . . . . . . . . . .

BIBLIOGRAPHY $\ldots \ldots \ldots \ldots \ldots \ldots \ldots \ldots \ldots \ldots \ldots \ldots \ldots \ldots \ldots$

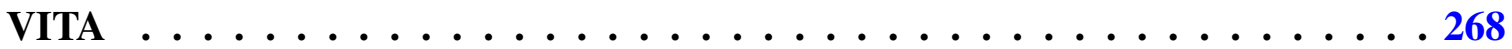




\section{LIST OF TABLES}

Table $\quad$ Page

2.1 Pearson correlations between the experimental SHAPE data and the 3DSSRpredicted SHAPE profiles using the different combinations of the three factors: interaction intensity $(I I)$, base pairing pattern $(B P)$, and ligand accessible surface $\left(\right.$ sas $\left.=S A S+S_{0}\right)$. Although the correlations generally decrease as sequences get longer, the log-scaled p-values, representing the statistical significance of our results, are displayed to show that our method

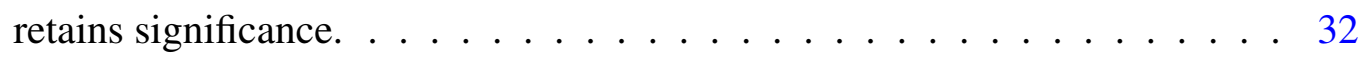

3.1 RNA structures used for validation. The Protein Database ID (PDBID), length of the RNA in nucleotides (nt), type of RNA, and organism of origin are displayed. The SHAPE profiles for these RNA molecules are from the published experimental data $[30,36,37,107,108] . \ldots \ldots \ldots \ldots$

3.2 Feature channels used for 3D images. . . . . . . . . . . . . . . . 64

3.3 Details of CNN Architectures. Each building block is shown with two convolutional layers. Downsampling is performed in every convolutional layer with a stride of $2 \ldots \ldots \ldots \ldots \ldots \ldots$. . . . . . . . . . . . . . . 65 
3.4 Pearson correlations between the experimental SHAPE data and the prediction algorithms: 3DSSR and the cross-validated CNN model trained on 11(19) cases and tested on the one left out, denoted as CNN12(CNN20). . . . . . . . . . . . 69

4.1 RNA structures used for validation of the model. The Protein Database ID (PDBID), length of the RNA in nucleotides (\#nt), type of RNA, and organism of origin are displayed. For RNA structures with multiple PDB entries in the database, we chose the one with the highest resolution. The SHAPE profiles for these RNA molecules are from the published experimental data [30, 36, 37, 108, 123]. * Structures used in new model that were not used to validate the previous 3DSSR model [78]. . . . 81

A.1 Parameter optimization using the leave-one-out approach. $E_{s t}$ and $b$ are in $\mathrm{kcal} / \mathrm{mol}, S_{0}$ is in $\AA^{2}$, and $a$ is dimensionless. . . . . . . . . . . 156

A.2 Experimental conditions of the SHAPE chemical probing for twelve RNAs. 157

A.3 Sequences, native 2D, and non-native 2D structures. . . . . . . . . . . 159

A.4 Sequences, native 2D, and non-native $2 \mathrm{D}$ structures. . . . . . . . . . . 160

A.5 Sequences, native 2D, and non-native $2 \mathrm{D}$ structures. . . . . . . . . . . 161

A.6 Sequences, native 2D, and non-native $2 \mathrm{D}$ structures. . . . . . . . . . . . 162

B.1 Table extracted from non-redundant structures in Basepair Catalog [129]. WatsonCrick, Hoogsteen, and sugar edges are denoted by W, H, and S, respectively. Cis and trans conformations are denoted by $\mathrm{c}$ and $\mathrm{t} . \ldots . . . . . . . . .170$

B.2 Physical descriptions for fit parameters in model. . . . . . . . . . . . 171

B.3 Sequences, native 2D, and non-native $2 \mathrm{D}$ structures. . . . . . . . . . . 172

B.4 Sequences, native 2D, and non-native 2D structures. . . . . . . . . . 173

B.5 Sequences, native 2D, and non-native 2D structures. . . . . . . . . . . 174 
B.6 Sequences, native 2D, and non-native 2D structures. . . . . . . . . 175

B.7 Sequences, native 2D, and non-native 2D structures. . . . . . . . 176

B.8 Sequences, native 2D, and non-native 2D structures. . . . . . . . . 177

B.9 Parameter optimization using the leave-one-out approach. The left out cases are labeled at the top. This table is continued on the next page, where averages and standard deviations for each parameter over all leave-one-out training runs are provided. $\ldots \ldots \ldots \ldots \ldots \ldots$

B.10 Continuation of parameter optimization using the leave-one-out approach from previous page. The left out cases are labeled at the top. . . . . . . 179

B.11 Sequences with SHAPE data used for reactivity bias calculation. . . . . . 180

B.12 Continued table of sequences with SHAPE data used for reactivity bias

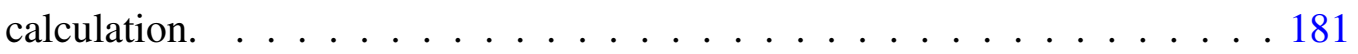

B.13 Continued table of sequences with SHAPE data used for reactivity bias

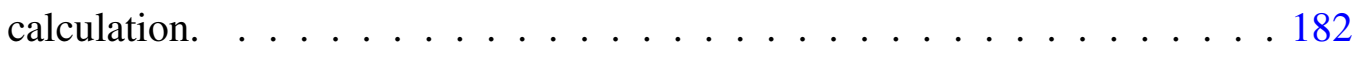

B.14 Performance comparisons between new model and 3DSSR using Spearman rank correlation (SRC), Pearson correlation of the near-native ensemble $\left(\mathrm{PC}_{\mathrm{NN}}\right)$, and Pearson correlation of the top-ranked structure $\left(\mathrm{PC}_{\mathrm{T}}\right)$. Information provided below is from the left out test RNA in each training run, showing that the performance improvement applies to structures the model has not seen. . . . . . . . . . . . . . . . . . . . 190

B.15 Estimated stability of $1 \mathrm{M} 7$ in the reactive site for various $\mathrm{XYZ}$ combinations. These free energies from REUS PMF calculations form the basis for Tables B.17

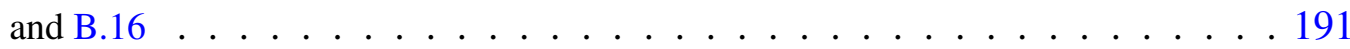


B.16 Stability in reactive site with respect to pairwise nucleotides from averaging over Table B.15. . . . . . . . . . . . . . . . . . . . . . . . 192

B.17 Stability in reactive site from single nucleotide averaging over Table B.15. . . . . 192

C.1 Thermal denaturation data for base pairing of $\mathrm{m}^{6} \mathrm{~A}$ compared with $\mathrm{A}$ from previous work [175]. Good agreement between two-state fits and van't Hoff plots indicates the duplexes exhibit two-state behavior. . . . . . . . . . . . . . . 194

C.2 Free energies $[\mathrm{kcal} / \mathrm{mol}]$ from eight runs. Estimated uncertainties from both trapezoidal thermodynamic integration (TI-T) and MBAR systematically underestimate the true uncertainty, calculated as the standard deviation of the values and shown in the "Ave." column. . . . . . . . . . . . . . . . . . . . . . . 202 


\section{LIST OF FIGURES}

Figure

1.1 An RNA hairpin viewed from different levels of analysis. a) The primary (1D) structure showing the sequence of nucleotides, oriented by the terminal functional groups in the chain: the 5'- and 3'-hydroxyl groups. b) The secondary (2D) structure showing the tetraloop and base-pairing interactions that form the stem. c) The tertiary (3D) structure showing the physical orientation of the nucleotides and atoms in the structure and the noncanonical base pair between the fourth and seventh nucleotides. . . . . . . . . . . . . . . . .

1.2 In this schematic of the SHAPE reaction, a magnesium ion quenches the negatively charged backbone phosphate oxygen and coordinates the reaction of the SHAPE ligand-1-methyl-7-nitroisatoic anhydride (1M7) — with the nucleotide through a bridging water molecule. A thick grey arrow shows the dipole moment of the $1 \mathrm{M} 7$ molecule. An arced arrow shows the movement of electrons from the 2'-hydroxyl oxygen to form a bond with the reactive carbon. Colored asterisks $(*)$ are placed near locations of functional groups whose relative distances are thought to affect the SHAPE reaction [32] . . . . . . . . . . . . . . 
1.3 Shown is adenosine (A) with one of its modified derivatives, $\mathrm{N}^{6}$-methyladenosine $\left(m^{6} \mathrm{~A}\right)$. This example methylation has major biological ramifications relating to obesity, memory consolidation, mental development, gene expression, and spermatogenesis $[53,54] \ldots \ldots \ldots \ldots \ldots$

1.4 Crystalline structure of CRISPR components based on Protein Database ID (PDBID) 5AXW. After recognition of the protospacer-adjacent motif (PAM) sequence (black), the first 20 nucleotide segment of the sgRNA pairs with the unwinding target DNA strand to form an RNA-DNA heteroduplex, and the non-target DNA strand is displaced inside the Cas9 protein (white). The lobes responsible for binding the sgRNA and DNA — recognition lobe (REC) — and for cleaving the DNA - the nuclease lobe (NUC) are also labeled. Credit: Thomas Splettstoesser (Wikipedia, CC BY-SA 4.0) . . . . . . . . . . . . . . . . . .

2.1 (a) The secondary structure of the Adenine riboswitch (PDB: 1Y26), the 3D structures of the helix, the 3-way junction and the hairpin-hairpin kissing motif. (b) Histogram of SHAPE reactivity [37] as a function of nucleotide position for 1Y26. The blue and red bars are for the nucleotides in helices and in loops/junctions, respectively. Not all the nucleotides in loops/junctions have high SHAPE reactivity, due to the constraints by other nucleotides and/or other molecules. . . . . . . . . 28 
2.2 Experimental SHAPE data is generally correlated with nucleotide dynamics. The scaled SHAPE reactivities [30, 36, 37] (shown in red) as a function of nucleotide position compared with the MD-estimated nucleotide flexibilities (scaled RMSD shown in black) for the highest correlated case (a) $2 \mathrm{~L} 8 \mathrm{H}$, the example case (b) 1Y26, and the lowest correlated case (c) $2 \mathrm{~L} 1 \mathrm{~V}$, respectively. (d) Ten snapshots of an MD simulation for 1Y26, with backbone phosphate $(\mathrm{P})$ atoms positionconstrained. The global fold of the RNA molecule is conserved, while each nucleotide has different fluctuations around its equilibrium state due to the constraints provided by other nucleotides. Highlighted in red are three nucleotides (U24, U36 and U50) that have large fluctuations and the highest SHAPE reactivity. The ligand molecules are not included during MD simulations. . . . . . . . . . . . 30

2.3 (a) Comparison between the predicted (black) and the experimental (red) SHAPE profiles for the TPP riboswitch (2GDI) [36], the case with the smallest p-value. (b) Comparison of SHAPE profiles for the TAR RNA (2L8H) [30], the case with the highest correlation. (c) Comparison of SHAPE profiles for the (small) M-Box Riboswitch (2L1V) [36], the case with the largest p-value. (d) Comparison of SHAPE profiles for the (large) M-Box Riboswitch (3PDR) [36], the case with the lowest correlation. . . . . . . . . . . . . . . . 35

2.4 Comparison of INF and correlation for the best (top) and worst (bottom) cases that were shown in Fig. 2.3. All the near-native MD cases (red), native 2D, non-native 3D cases (black), and 3D structures with non-native 2D structure (magenta) are displayed for each case. These results show that exclusion of most non-native 2D structures and many non-native 3D structures is possible using the 3DSSR model. 
2.5 (a) Comparison between the 3DSSR-predicted (black) and experimental (red) SHAPE profile for the adenine riboswitch (1Y26) [37], which has a Pearson correlation of 0.88. (b) Comparison between the predicted (black) and experimental (red) SHAPE profile for the cyclic-di-GMP riboswitch from V. cholera (3IWN) [36], which has a Pearson correlation of 0.74. Nucleotides marked (1) and (2) have high predicted SHAPE reactivity due to the base-ligand interactions that are not considered in the 3DSSR model. Nucleotides marked (3) are in helix region but have medium SHAPE reactivity. The false prediction for the nucleotide marked (4) is likely due to the effect of the tail not considered in the current 3DSSR function. . $\quad 42$ 
2.6 In this reaction mechanism, a magnesium ion quenches the negatively charged backbone phosphate group and simultaneously coordinates the reaction of the $1 \mathrm{M} 7$ ligand with the nucleotide through a bridging water molecule. A thick grey arrow shows the dipole moment going through the 1M7 molecule, which points from the partially anionic oxygen bound to the reacting carbon toward the partially cationic ring nitrogen. If bound to an ideally positioned magnesium ion, a coordinating water molecule may weakly hydrogen bond to the partially anionic oxygen on the $1 \mathrm{M} 7$ molecule and facilitate increased sampling of SHAPE-reactive space. The magnesium ion may anchor $1 \mathrm{M} 7$ in a reactive orientation so that the reactive carbon collides with the 2'-hydroxyl oxygen until the reaction occurs. If the negatively charged phosphodiester backbone is not quenched, the polarized $1 \mathrm{M} 7$ molecule would be more likely to sample the reactive space in an unfavorable (nonreactive) orientation. An arced arrow shows the movement of electrons from the 2'-hydroxyl oxygen to form a bond with the reactive carbon. In this picture, the magnesium ion behaves as a kinetic catalyst by providing a means for SHAPE ligands to more frequently sample the reactive space in a favorable orientation, and it acts as a weak chemical catalyst by pulling electrons from the partially negative oxygen through the hydrogen bond with water and increasing the reactivity of the

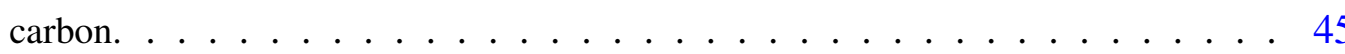


2.7 (a) 3D structures for some of the interactions not considered in the 3DSSR function: base-ligand interactions for (1) and (2) in Fig. 2.5; non-WWc stacking for (5) in Fig. 2.7b and base-backbone interactions between the nucleotide G62 and nucleotides G9-G11 (6) in the TPP riboswitch from E. coli (2GDI). (b) Comparison of the predicted (black) and experimental (red) SHAPE profile for the lysine riboswitch from T. maritime (3DIG) [36], which has a Pearson correlation of 0.70 . The false prediction for the nucleotides marked (5) is likely caused by the underestimation of the interaction intensity within the non-WWc stacking region shown

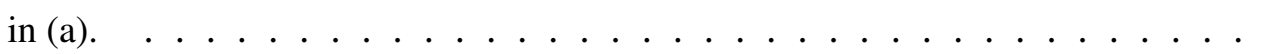

3.1 The SHAPE reaction. The RNA nucleotide 2'-OH group attacks the reactive carbon of $1 \mathrm{M} 7$, releases $\mathrm{CO}_{2}$, and forms a covalent bond (purple) with the SHAPE

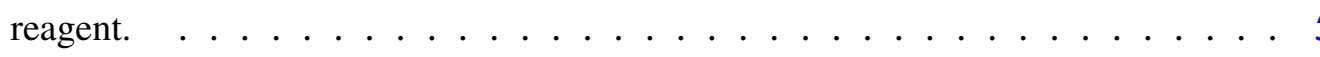

3.2 The 2D, 3D, and SHAPE reactivity of RNA-Puzzle 8 (PDBID: 4L81). A) The 2D structure [111] shows the four-way junction (4WJ), base-pairs, and long range interactions. B) The 3D structure shows the $4 \mathrm{WJ}$ and an example of a base stacking interaction. C) The experimental and predicted SHAPE profiles for the crystallized 4L81 structure show good agreement $($ Pearson correlation $=0.57) \ldots \ldots$. . . . . 57

3.3 3DSSR workflow on an RNA-Puzzle 8 decoy. A) A candidate 3D structure decoy is processed by RNApdbee 2.0 [112], RNAview [9], in-house software, and VMD [85] to B) produce a 2D structure, C) identify base pair types, D) extract stacking angle/distance information, and E) calculate the solvent accessible surface of the 2'-OH, respectively. The information extracted from the structure is input into the 3DSSR function to produce F) the predicted SHAPE profile for each

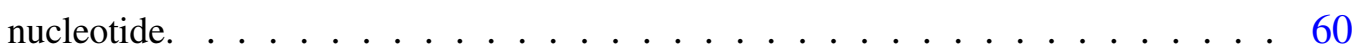


3.4 Extracting the 3D image of an RNA nucleotide. The magenta color depicts the nucleotide under assessment and the surrounding environment is confined within the cube with length $24 \AA$. The surrounding atoms are drawn in cyan, and the cube boundaries are drawn with yellow solid lines. . . . . . . . . . . . 63

3.5 Sieving SHAPE-incompatible structures from RNA-Puzzle 8 submissions. A) The 3D representation shows the trend of the assessment results (RMSD and INF) with the correlation between 3DSSR-predicted SHAPE profiles and experimental SHAPE (3DSSR Correlation). Warmer colors indicate higher correlation, higher INF, and lower RMSD. The INF and RMSD values were taken from the RNAPuzzles database. 2D plots of the B) INF and C) RMSD with respect to the 3DSSR and CNN20 correlations are also shown, along with their respective Spearman rank coefficients $(\mathrm{SR}) . \ldots \ldots \ldots \ldots \ldots$

4.1 Details of stacking and base pairing calculations. To clarify the details of the stacking and base pairing calculation, we zoom into a section of a validation structure. Nucleotide $84 \mathrm{G}$ is indexed as the $a(i)$ nucleotide and is base pairing (stacking) with $112 \mathrm{C}(84 \mathrm{G})$. The $84 \mathrm{G}-112 \mathrm{C}$ base pair is a cis-Watson Crick base pair $(t=\mathrm{cWW})$. In the $84 \mathrm{G}-85 \mathrm{G}$ stack, the $\mathrm{C} 2, \mathrm{C} 4$, and C6 atoms are highlighted in magenta, and the distance (d) and angle $(\theta)$ between the planes are noted. As long as the stack meets the cutoffs described in the example calculation, we include the 3' nucleotide typedependent stacking contributions in the energy score. . . . . . . . . . . . 77 
4.2 Bar chart showing performance differences. For each validation structure in leave-one-out training, the difference between the new model and 3DSSR model performance is shown. Near-native (top-ranked) structure PC performance differences are denoted 'NN' ('Top'). Average performance comparisons are at the top of the chart, denoted 'Ave'. Positive differences indicate higher performance by the new model. Absolute performance comparisons can be found in Table B.14 in Appendix B. . . . . . . . . . . . . 95

4.3 Plot showing trends between PC, INF, and RMSD for the longest validation structure, 3DIG. Each of the 90 points in magenta (red) represents a randomly selected near-native decoy from an all-atom simulation with base pairing (only backbone) restraints. Each of the 90 (450) points in cyan (green) represents a randomly selected non-native 3D (2D) decoy from a CG simulation. As the INF (RMSD) increases (decreases), the PC between SHAPE data and our prediction increases. The spectrum of results shows how our model can use SHAPE to select near-native 3D structures and indicates that SHAPE contains important tertiary structure information, especially for long structures. Shadows are projected onto surfaces for 2D

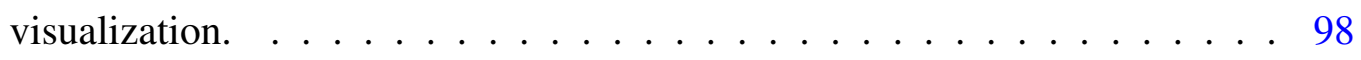


4.4 SHAPE bias energy score compared with stability of unbound ligand in binding pocket. The SHAPE bias energy score (y-axis) is directly correlated to the free energy change relative to the average calculated from REUS simulations (x-axis). The SHAPE reactivity cutoff used for the bias calculation was $\xi=0.1$. Blue (red) data points relate the pairwise bias to the REUS-calculated free energy change, influenced by the previous (next) nucleotide type, $\mathrm{X}(\mathrm{Z})$, and the NOI, Y. Inherent low reactivity of SHAPE to $\mathrm{C}$ is accounted for by a $0.4 \mathrm{kcal} / \mathrm{mol}$ penalty in the REUS calculation. . . 101

5.1 Coarse-grained descriptions of canonical A, U, G, C nucleotides. The backbone is represented by beads located at the $\mathrm{P}$ and $\mathrm{C} 4$ ' atoms (denoted $\mathrm{S}$ for the related ribose sugar). Purine (A and $G$ ) bases are represented by three beads each $\left(R_{C}\right.$, $\left.A_{C}, A_{N}\right)$ and $\left(R_{C}, G_{O}, G_{N}\right)$. Pyrimidine ( $U$ and $C$ ) bases are represented by two beads each $\left(\mathrm{Y}_{\mathrm{C}}, \mathrm{U}_{\mathrm{O}}\right)$ and $\left(\mathrm{Y}_{\mathrm{C}}, \mathrm{C}_{\mathrm{N}}\right)$. $\mathrm{CG}$ beads for the bases are centered at the corresponding heavy-atom group center-of-mass. . . . . . . . . . . . . 112 
5.2 Correlated folding coordinates lead to same final energies regardless of parameterization order. A) After incorporating bond length information in our reference force field, information about the $S-R_{C}-G_{O}$ bond angle $\theta_{1}$ still needs to be included because the reference simulation does not capture the $\theta_{1}$ distribution. However, a correlated $\mathrm{G}_{\mathrm{O}}-\mathrm{R}_{\mathrm{C}}-\mathrm{G}_{\mathrm{O}}$ angle $\theta_{2}$ is fully captured by the reference force field without being explicitly included in the force field. B) As we include more folding

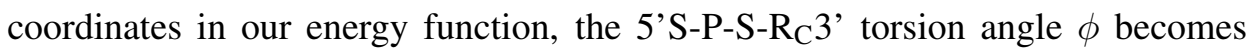
more similar to the observed distribution, indicating correlation between $\phi$ and the previously included structure factors. C) and D) Although the individual factors are correlated, the final energy function yields the same final energies, regardless of parameterization order. In C), the $r_{1}$ folding coordinate is incorporated into the force field, followed by $r_{2}$. In D), the parameterization order is reversed, but the final total energies and distributions produced by simulation are unchanged. . . . 116

5.3 Incorporating the first non-local pairwise folding coordinate. After all the required local folding coordinates have been included in $E_{\text {ref }}\left(r_{s}\right)$, non-local base pairing effects can be accounted for. Once this collective variable is included, the energy function produced by simulation $E_{\mathrm{sim}}\left(r_{s}\right)$ matches the observed distribution in the structure database $E_{\mathrm{obs}}\left(r_{s}\right) \ldots \ldots \ldots \ldots 18 \ldots \ldots \ldots$

5.4 A selection of extracted CG energy functions using the IsRNA method. The left and middle columns show non-local energy functions between nucleotides. The right column shows the energy function for a local bond length $b$, torsion angle $\phi$, and bond angle $\theta \ldots \ldots \ldots \ldots \ldots \ldots \ldots$ 
5.5 Application of the IsRNA model in RNA folding simulations and 3D structure predictions for (A) the helix II of Xenopus laevis 5S rRNA with a cytosine bulge (PDB ID: 1DQF) and (B) the CUUG hairpin loop (PDB ID:1RNG). From left to right: the secondary structure extracted from the native structure, the predicted 3D structure (red color) superposed to the native structure (blue color), and the plot of RMSDs with potential energies in the folding simulation. . . . . . . . 126

6.1 Unmodified 2D and 3D structures. For the A) duplex and B) hairpin, analogous regions are highlighted in magenta and the experimentally probed base pair is denoted with a blue arrow. Nucleotides are numbered from 5', to 3 ' ends. . . . . . . . . . . . . . . . . . . . . 135 
6.2 A thermodynamic cycle formed by the states indicated by red arrows. In order to separate the electrostatic and Lennard-Jones (LJ) contributions to the free energy change, we use three main steps to go from the WT to the modified states. The first step, labeled "Discharge", removes all charge from the atoms in the starting residue. The blue residues are electrically neutral WT residues. The second step, labeled "LJ", replaces atoms and bonds in the WT residue with atoms and bonds from the modified residue. Because the vanishing and appearing atoms are all electrically neutral in this step, the LJ potential is the contributor to the free energy change. The green residues are electrically neutral, modified residues. The third step, labeled "Charge", reintroduces charge to the modified residue to bring us to the final, modified state. The red residues represent the electrically charged, modified residues. These steps must be done for both the duplex and reference (single stranded) states in order to calculate the relative free energy change due to modification $\Delta \Delta G \ldots \ldots \ldots$. . . . . . . . . . . . .

6.3 Calculated relative free energy changes using TI-Trapezoid. Experimental [175] (calculated) results and error bars are shown in black (red). The results for twice-perturbed cases are the average of the results from the modify-then-mutate and the mutate-then-modify alchemical paths. Estimates from applying the Turner rules are shown in blue [212]. . . . . . . . 142 
6.4 Experimental [175] and calculated relative free energy changes due to modification $\Delta \Delta G$. For the eight base pair duplex, experimentally determined free energies calculated from two-state curve fits, van't Hoff linear plots, and averages between the two-state fit and van't Hoff fit are labeled "2-state fit", "van't Hoff", and "Exp. average", respectively. The values for $\Delta \Delta G$ calculated from the RMSD/E2E 2D PMFs of the hairpin ana$\log$ are labeled "Calc. from PMF". Descriptive identifiers are provided to describe modifications and/or mutations (see Fig. 6.1): for example, " $\mathrm{m}^{6} \mathrm{~A}$ $\mathrm{U}$ " represents the modification of $2 \mathrm{~A}(5 \mathrm{~A})$ to $\mathrm{m}^{6} \mathrm{~A}$ in the simulated hairpin (experimental duplex), maintaining the canonical base pair with 9U (12U). Likewise, "A-C" represents the hairpin (duplex) mutation of 9U (12U) to C. All hairpin (duplex) mutations were imposed on 9U (12U). The gray line indicates the free energy plateau, where the hairpin becomes completely destabilized. Experimental error bars are shown with respect to the experimental average. Estimates from applying the Turner rules are shown in blue. . . . . . . . . . . . . . . . . . . . . . . . . . . . 145

6.5 2D PMFs and representative frames from hairpin TREMD simulations. The representative frames and PMFs for the A) WT B) modification of $2 \mathrm{~A}$ to $\left.\mathrm{m}^{6} \mathrm{~A}\left(2 \mathrm{~m}^{6} \mathrm{~A}\right), \mathrm{C}\right)$ modification of $5 \mathrm{U}$ to $\left.\mathrm{m}^{5} \mathrm{U}\left(5 \mathrm{~m}^{5} \mathrm{U}\right), \mathrm{D}\right)$ modification of $7 \mathrm{G}$ to $\mathrm{m}^{2} \mathrm{G}\left(7 \mathrm{~m}^{2} \mathrm{G}\right)$ are shown along the end-to-end distance (E2E) and RMSD reaction coordinates. Modified carbons and ribbons in the representative frames are highlighted in green, and the figure legends color code the energy in $\mathrm{kcal} / \mathrm{mol} . \ldots \ldots \ldots$ 
A.1 The 1M7-nucleotide bound conformation, built based on two similar ligands in the PDB database (PubChem CID 12076 and 66345). The effective ligand radius is measured using the Van der Waals surface shown in the figure. . . . . . . . . 155

A.2 Comparison between the predicted (black) and the experimental (red) SHAPE pro-

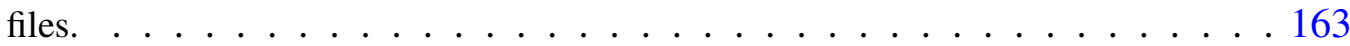

A.3 Comparison between the nucleotide RMSD (black) and the experimental SHAPE (red) profiles. . . . . . . . . . . . . . . . . . . . . . 164

A.4 Comparison of INF and correlation for the first six cases. Near-native structures from all-atom MD sampling are red; native 2D, non-native 3D structures from CG sampling are black; non-native 2D, non-native 3D structures from CG sampling are magenta. . . . . . . . . . . . . . . . . 165

A.5 Comparison of INF and correlation for the last six cases. Near-native structures from all-atom MD sampling are red; native 2D, non-native 3D structures from CG sampling are black; non-native 2D, non-native 3D structures from CG sampling are magenta. . . . . . . . . . . . . . . . . . 166

A.6 Comparison of RMSD and correlation for the first six cases. Near-native structures from all-atom MD sampling are red; native 2D, non-native 3D structures from CG sampling are black; non-native 2D, non-native 3D structures from CG sampling are magenta. . . . . . . . . . . . . . . . . . 167

A.7 Comparison of RMSD and correlation for the last six cases. Near-native structures from all-atom MD sampling are red; native 2D, non-native 3D structures from $C G$ sampling are black; non-native 2D, non-native 3D structures from CG sampling are magenta. . . . . . . . . . . . . . . . . . . 168 
B.1 1M7 ligand in initial complex with the 5'UAAAU3' 5-mer. Since the ligand is initially positioned near the 2'-hydroxyl of the third nucleotide, it is between the third and fourth bases, which predisposes the SHAPE reagent to travel toward the 3 ' end of the 5 -mer. . . . . . . . . . . . . . . . . . 183

B.2 PMF and various scatter density plots for normal umbrella sampling of the 5'UAAAU3' 5-mer. The $\mathrm{x}$-axis for each panel is the distance from the 2'-oxygen of the $3 \mathrm{~A}$ (Y in UXYZU 5-mer) nucleotide to reactive carbon of 1M7. The left y-axis for each panel is the PMF energy. The right $y$-axis for each panel is the distance from the 2'-oxygen of the 5' $\mathrm{U}, \mathrm{X}, \mathrm{Z}$, and 3' $\mathrm{U}$ nucleotides to the reactive carbon of $1 \mathrm{M} 7$. The 1M7 ligand tends to heavily travel toward the 3' end of the 5-mer and become trapped. The jagged PMF shows that transition state sampling in normal US is

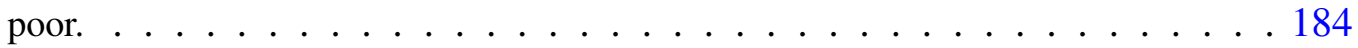

B.3 PMF and various scatter density plots for REUS of the 5'UAAAU3' 5-mer. The $\mathrm{X}$-axis for each panel is the distance from the 2'-oxygen of the $3 \mathrm{~A}(\mathrm{Y}$ in UXYZU 5-mer) nucleotide to reactive carbon of 1M7. The left y-axis for each panel is the PMF energy. The right y-axis for each panel is the distance from the 2'-oxygen of the 5'U, X, Z, and 3'U nucleotides to the reactive carbon of $1 \mathrm{M} 7$. Although the ligand still tends to travel toward the 3' end, the sampling is much more uniform with the REUS implementation, and the transition states are lower energy than indicated by the normal US implementation, indicating improved transition state

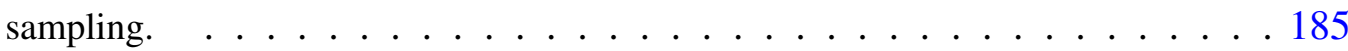

B.4 Density histograms of the umbrellas for REUS with the 5'UAAAU3' sequence. Good overlap is observed with these biasing umbrellas, and the approximate normal distributions of the umbrellas indicates sufficient spring stiffness. . . . . . 186 
B.5 PMF as a function of reaction coordinate distance from averaging all 5'UXYZU3' REUS runs. The average PMF provides a reference state and allows us to establish the relative binding stability of the 1M7 ligand in different sequence contexts. . . 187

C.1 Melting curve . . . . . . . . . . . . . . . . . . . 203

C.2 Comparing free energy estimators $\ldots \ldots \ldots \ldots \ldots \ldots \ldots$

C.3 Confirming convergence of TREMD PMFs $\ldots \ldots \ldots \ldots \ldots$

C.4 Example overlap matrix . . . . . . . . . . . . . . . . . . 213

C.5 Example convergence plots . . . . . . . . . . . . . . . . . 214

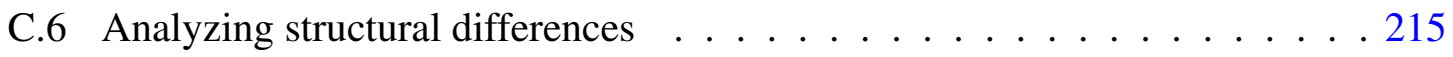

C.7 Duplex conformation compared to single stranded . . . . . . . . . 216

C.8 RMSD/E2E PMF and representative frames for WT . . . . . . . . . 217

C.9 RMSD/E2E PMF and representative frames for $2 \mathrm{~m}^{6} \mathrm{~A} \ldots \ldots \ldots \ldots$

C.10 RMSD/E2E PMF and representative frames for $3 \mathrm{~m}^{4} \mathrm{C} \ldots \ldots \ldots$

C.11 RMSD/E2E PMF and representative frames for $4 \mathrm{~m}^{5} \mathrm{U} \ldots \ldots \ldots \ldots . .222$

C.12 RMSD/E2E PMF and representative frames for $5 \mathrm{~m}^{5} \mathrm{U} \ldots \ldots \ldots \ldots .223$

C.13 RMSD/E2E PMF and representative frames for $6 \mathrm{~m}^{4} \mathrm{C} \ldots \ldots . . .225$

C.14 RMSD/E2E PMF and representative frames for $7 \mathrm{~m}^{2} \mathrm{G} \ldots \ldots \ldots . .226$

C.15 RMSD/E2E PMF and representative frames for $8 \mathrm{~m}^{2} \mathrm{G} \ldots \ldots \ldots \ldots .228$

C.16 RMSD/E2E PMF and representative frames for $9 \mathrm{~m}^{5} \mathrm{U} \ldots \ldots \ldots . . .230$ 


\begin{abstract}
Ribonucleic acid (RNA) is a polymeric nucleic acid that is crucial for cellular function, regulating gene expression and encoding/decoding protein/DNA molecules. Recent discoveries of diverse functionality in non-coding RNAs have led to unprecedented demand for RNA 3D structure determination. With current technology, general, accurate prediction of 3D structures for large RNAs from the sequence remains computationally intractable. One of the principal challenges arises from the conformational flexibility of RNA, especially in loop/junction regions, which results in a rugged energy landscape. Several strategies exist to overcome this challenge, including incorporation of efficient experimental information and coarse-grained (CG) modeling to improve computational sampling of the structural ensemble. A second challenge is the inclusion of naturally modified derivatives of canonical RNA nucleotides in structure analysis. Most RNA prediction strategies rely upon the canonical nucleotides (adenine (A), uracil (U), guanine $(\mathrm{G})$, and cytosine $(C)$ ), ignoring the effects of modified nucleotides on the structure and system dynamics.

In general, RNA molecules contain rigid and flexible structural elements, which can be probed using efficient selective 2'-hydroxyl analyzed by primer extension (SHAPE) experiments. SHAPE experiments selectively modify flexible RNA nucleotides and can be processed to produce a characteristic reactivity profile for an RNA molecule that contains structural information. Incorporation of efficient experimental information, such as SHAPE, in predicting RNA 3D structure is highly desirable for overcoming the current knowledge gap between RNA sequence and 3D structure. In the first project, we introduce a physics-based model, the 3D structure-SHAPE relationship (3DSSR) model, to predict the SHAPE reactivity from the structure and show how this model may be used to sieve
\end{abstract}


SHAPE-compatible structures from a pool of low-energy decoys and refine our predictions. In the second project, we compare 3DSSR performance to that of a convolutional neural network $(\mathrm{CNN})$ trained on the SHAPE data and RNA structures, showing that 3DSSR outperforms the $\mathrm{CNN}$ given the limited data available. In the third project, we further improve the 3DSSR model, gaining deeper insights into the SHAPE reaction and biases.

In the fourth project, we explore the theory underpinning the iterative simulated CG RNA folding model (IsRNA). In establishing the underlying mechanics driving the success of the model, we were able to clarify and improve the parameterization method while expanding the model interpretation, which should broaden application of the method to other biopolymers, such as protein. We found that the parameterization method follows statistical mechanics principles but also has a Bayesian interpretation. Further, we found that the parameterization process can benefit from application of the principle of maximum entropy, which improves simulation and parameterization efficiency.

In the fifth project, we investigate the impact of nucleotide modification on the structure and configurational ensemble of RNA molecules using free energy calculations. By applying modifications to a common RNA hairpin, we estimate the impact on the stability of the structural ensemble, identifying specific interactions that drive changes to the potential of mean force (PMF) and showing the context and modification-dependence of the variable alterations to the structure stability. 


\section{Chapter 1}

\section{Introduction}

The backgrounds of my research projects are described in this chapter.

\subsection{Reviewing RNA fundamentals}

Ribonucleic acid (RNA) is a polymeric nucleic acid that plays crucial roles in cellular functions by regulating gene expression and coding/decoding protein and DNA. The fundamental element of RNA is the nucleotide, which is made up of a ribose sugar and a nucleobase, such as adenine $(A)$, cytosine $(C)$, guanine $(\mathrm{G})$, or uracil $(\mathrm{U})$. The nucleotides are connected through phosphodiester bonds to form RNA. RNA has many fundamental features similar to deoxyribonucleic acid (DNA). However, RNA tends to exist in single stranded states that form a variety of intrastrand interactions, whereas DNA primarily exists in double stranded states and forms long duplexes. RNA structure is understood through multiple levels of analysis: in the primary structure (1D), we can view the sequence of nucleotides that form an RNA strand; in the secondary structure (2D), we can visualize the close-range 
a)

$$
\text { 5'-GACUUCGGUC-3' }
$$

c)

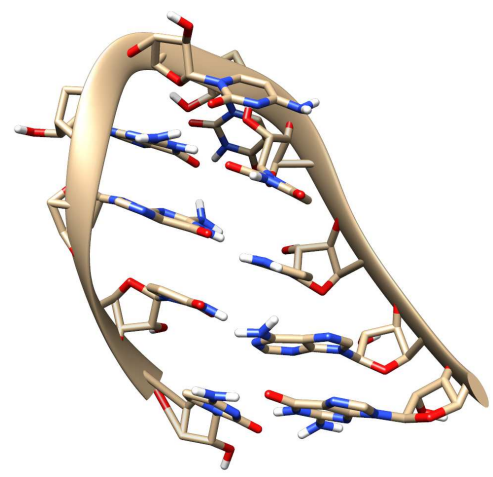

b)

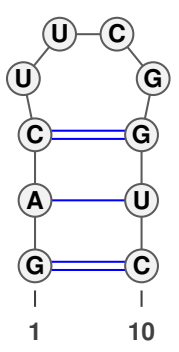

Figure 1.1: An RNA hairpin viewed from different levels of analysis. a) The primary (1D) structure showing the sequence of nucleotides, oriented by the terminal functional groups in the chain: the 5'- and 3'-hydroxyl groups. b) The secondary (2D) structure showing the tetraloop and base-pairing interactions that form the stem. c) The tertiary (3D) structure showing the physical orientation of the nucleotides and atoms in the structure and the noncanonical base pair between the fourth and seventh nucleotides.

intrastrand interactions formed by base-pairing; in the tertiary structure (3D), we can visualize spatial geometries and all of the interactions that form the physical structure of an RNA (see Fig. 1.1). Important information can be extracted from all of the varying levels of analysis, which improves the understanding of RNA structure, dynamics, and function.

Recent discoveries concerning new functions of non-coding RNAs have led to an unprecedented rise in demand for the determination of RNA 3D structures [1, 2]. However, accurate computational prediction of RNA 3D structures from the sequence remains a significant unsolved problem [1-3]. One of the principal challenges arises from the rugged energy landscape. Base pairing between different nucleotides leads to the formation of different secondary structures consisting of different helices and loops. Many secondary structures can be thermodynamically stable/metastable, corresponding to local minima on the energy landscape. Moreover, for a given secondary structures, the conformational flex- 
ibility, especially in the loop and junction regions, and the intraloop interactions between the nucleotides result in further rugged energy landscape of RNA. The dynamic structures of RNA lead to the diverse catalytic and regulatory roles of RNA in cellular functions [4, 5] such as transcription, mRNA splicing, and translation [6, 7]. RNA nucleotides contain a ribose sugar on the backbone chain with a base attached to the 1' position of the sugar ring. Phosphodiester backbone rotation facilitates large conformational flexibility of RNA [8], which allows sampling of a large portion of the conformational space. RNA bases form a variety of strong base-base and base-backbone interactions [9-11], which leads to a multitude of stable and metastable RNA conformations. The distribution of the collection of heterogeneous conformations determines RNA structure and stability.

In general, RNA structures contain both rigid and flexible structural elements. The rigid structural elements such as RNA helices and structured loops are primarily stabilized by base-base interactions, such as base-pairing and stacking in secondary structures and other noncanonical and long-range interactions in tertiary structures. Both global structural features, such as helix orientations, and local structural features are essential for the interpretation of RNA structural changes in function, such as gene regulation through ligand-induced RNA conformational switches and protein-induced structural changes at active sites.

From an energetics point of view, a flexible RNA can adopt multiple low-energy states from sampling a variety of global folds and local conformations [12-14]. Low-energy state multiplicity results in great difficulties for predicting and modeling RNA 3D structures. For instance, because of the diversity and sequence-sensitivity of loop/junction structures, 3D structure predictions often rely on fragment/template assembly for highly homologous sequences [15-21]. However, given the limited number of known RNA structures, structural motif templates with the required high sequence identity are difficult to attain. Of the 
structures in the PDB database, few $(<5000)$ are high-resolution crystal structures $(<3 \AA)$. The lack of reliable structural templates for loops and junctions greatly hampers accurate structure prediction.

\subsection{Examining significance of SHAPE probes}

Inspired by the recent progress in RNA chemical probing methods, researchers have proposed and developed several data-facilitated experimental modeling approaches to complement established template and physics-based methods [22-26]. Selective 2'-hydroxyl acylation analyzed by primer extension (SHAPE) is an RNA structure probing technology that provides significant insights into local nucleotide structure and dynamics [27, 28]. SHAPE reagents are small ligands-1-methyl-7-nitroisatoic anhydride (1M7), 1-methyl-6nitroisatoic anhydride (1M6), N-methylisotoic anhydride (NMIA), benzoyl cyanide (BzCN), 2-methyl-3-furoic acid imidazolide (FAI), and 2-methylnicotinic acid imidazolide (NAI)— that form a 2'-O-adduct with the nucleotide 2'-hydroxyl group [29]. Previous studies [3032] suggested that unconstrained nucleotides that sample more flexible conformations have greater probability to adopt the SHAPE-reactive conformations and, thus, show higher SHAPE reactivity. In contrast, nucleotides that are constrained by base-pairing and stacking interactions are much less reactive. This quantitative measurement of local nucleotide dynamics makes SHAPE an effective tool to probe whether a nucleotide forms a base pair (either in a helix or in a structured loop) or remains unpaired in a flexible loop/junction. In comparison to modeling without experimental data, SHAPE data places effective constraints on RNA conformational space and can significantly improve the efficiency of conformational sampling. SHAPE-directed RNA structure prediction substantially increases 


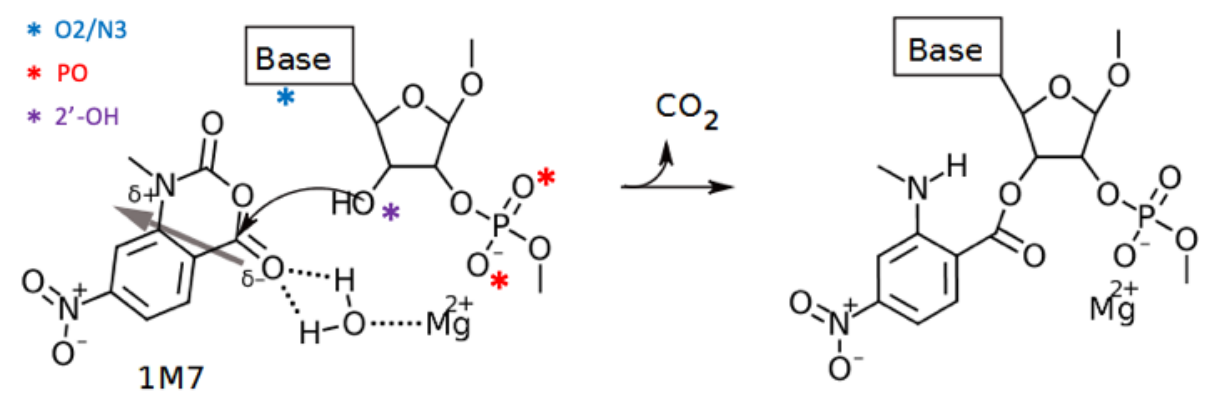

Figure 1.2: In this schematic of the SHAPE reaction, a magnesium ion quenches the negatively charged backbone phosphate oxygen and coordinates the reaction of the SHAPE ligand-1-methyl7-nitroisatoic anhydride (1M7) — with the nucleotide through a bridging water molecule. A thick grey arrow shows the dipole moment of the $1 \mathrm{M} 7$ molecule. An arced arrow shows the movement of electrons from the 2'-hydroxyl oxygen to form a bond with the reactive carbon. Colored asterisks (*) are placed near locations of functional groups whose relative distances are thought to affect the SHAPE reaction [32].

the accuracy of RNA secondary structure modeling [33-37], where SHAPE reactivity/nucleotide dynamics provides additional structural constraints for the free-energy based predictions [38]. Moreover, combined with other strategies, such as differential SHAPE reactivity, mutate-and-map, and time-resolved SHAPE chemistry, SHAPE probing provides highly useful information for the in vitro and in vivo determination of non-canonical tertiary interactions and RNA kinetics[39-45].

Although SHAPE technology is widely applied and is a highly valuable tool for RNA modeling and (2D) structure prediction, the mechanism of the SHAPE reaction is not fully understood. A variety of conformations render a nucleotide reactive by SHAPE [33, 34]. Specific RNA structural features correspond to high SHAPE reactivity, including a long PO-to-2'-OH distance, short 2'-OH to $\mathrm{O} 2 / \mathrm{N} 3$ distance, and short 2'-OH to non-bridging $\mathrm{O}$ distance (see Fig 1.2). These conclusions [32] were mainly derived from SHAPE mapping data for 16S rRNA in ribosome crystals and focused on conformations that tend to increase SHAPE reactivity. Long PO-to-2'-OH distance is thought to facilitate SHAPE reaction by stabilizing the formation of an anionic $2^{\prime}-\mathrm{O}^{-}$intermediate, while the short distances be- 
tween basic groups and the 2'-OH are thought to enhance the SHAPE reaction by stripping the 2'-OH proton. Alternatively, we have proposed that SHAPE reactivity may be enhanced by a magnesium ion quenching the negatively charged backbone and increasing sampling of SHAPE-reactive space by the polarized SHAPE ligand (see Fig. 1.2). Low SHAPE reactivities are known to correlate with positions in helices and rigidly structured loops, but the full mechanisms by which nucleotides can be conformationally constrained, and rendered inert, are not fully understood. Additionally, the accuracy of SHAPE experiments relies on carefully chosen experimental and data processing methods [35, 37]. While SHAPE data may suggest that an unreactive nucleotide forms an interaction with another member of the system, SHAPE cannot give us information about who is interacting with the nucleotide or what kind of interaction is formed[35]. These limitations mean that SHAPE-directed structures should not be considered absolutely determined, but SHAPE can give us useful structural hypotheses[35]. Although SHAPE has limitations, prediction of 2D structure using SHAPE has reached a level of accuracy on par with comparative sequence analysis [33]. Conversely, by calculating the effective stability determined by O2'-P-O5' angle fluctuations and optimizing the native structure-based potential using MD simulations, SHAPE profiles have been rebuilt [46].

\subsection{Using coarse-grained simulations to fold RNA}

While knowledge-based, template-fitting methods can rapidly predict RNA structures with phylogenetic similarities to existing structures, they are limited by the availability of known structures and fragments and do not provide much information about the system dynamics [47]. Unlike these template-based methods, all-atom molecular dynamics (MD) simu- 
lations do not rely on existing templates because they are constructed from first principles, and they can be used to detail folding dynamics [48, 49]. However, for lengthy RNA, allatom MD simulations on the scale of milliseconds or seconds is intractable due to current limitations $[50,51]$.

To overcome these limitations while taking advantage of the respective strengths, we turn to coarse-grained (CG) molecular dynamics (CGMD) simulations, which provides compromises between these methods in terms of speed, accuracy, and information content. Representing groups of atoms with CG pseudobeads facilitates acceleration of the simulations, smoothing out the rugged RNA energy landscape and choosing only those degrees of freedom that greatly contribute to the system dynamics [52].

We previously reported the Iterative simulated RNA reference state (IsRNA) method for parameterizing a CG force field for RNA folding [53], which has two main advantages. Firstly, the model accounts for correlation effects through consecutive simulations of a reference state as new information is included in the force field. Secondly, the IsRNA model accounts for non-native interactions in RNA folding. These advantages allow the force field to reproduce RNA observables and known 3D structures with alacrity.

\subsection{Assessing significance of modified nucleotides}

Motivated by recent discoveries regarding the fundamental role of RNA molecules in myriad essential functions within the cell and equipped with groundbreaking experimental detection techniques, experimental discovery of unique RNA sequences and nucleotide modifications has impressively escalated (see Fig. 1.3 for an example of nucleotide modification) [55]. While the existence of modified nucleotides and the need for nucleotide modifi- 

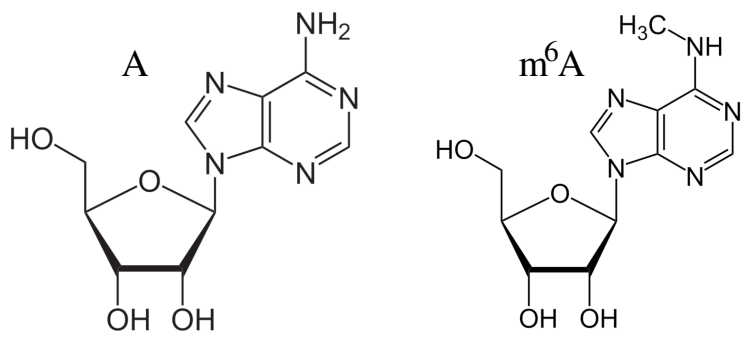

Figure 1.3: Shown is adenosine (A) with one of its modified derivatives, $\mathrm{N}^{6}$-methyladenosine $\left(\mathrm{m}^{6} \mathrm{~A}\right)$. This example methylation has major biological ramifications relating to obesity, memory consolidation, mental development, gene expression, and spermatogenesis [53, 54].

cation to ensure stability of functional RNA structure has been long known $[56,57]$, the recent improvement of sequencing and detection technologies in the field of epitranscriptomicsnatural, post-transcriptional modifications to RNA - has sparked discoveries relating dynamic modification in both coding and non-coding RNA to diverse modulation of RNA structure, cellular function, and gene expression [55, 58, 59]. Although understanding the effect of nucleotide modification on RNA structure and function is highly relevant to advancing treatment of common medical conditions-including cancer, neurological disorders, diabetes, mitochondrial diseases [60], and HIV [61] - experimental determination of RNA 3D structures remains labor-intensive and difficult due to the dynamical nature of RNA molecules, the negatively charged phosphate backbone, and the difficulty of finding crystal contacts $[62,63]$. Because of new sequencing techniques and crystallographic challenges, the discovery of unique RNA sequences has rapidly outpaced experimental determination of corresponding 3D structures. To help address the gap between sequencing data and structural information, accurate computational prediction of RNA 3D structure is an important problem and necessary approach for RNA structure determination.

In addition to learning how natural nucleotide modifications affect RNA structure and how these structural changes affect the function, we also wish to understand how controlling these modifications can aid in development of personalized medicine technology. For 


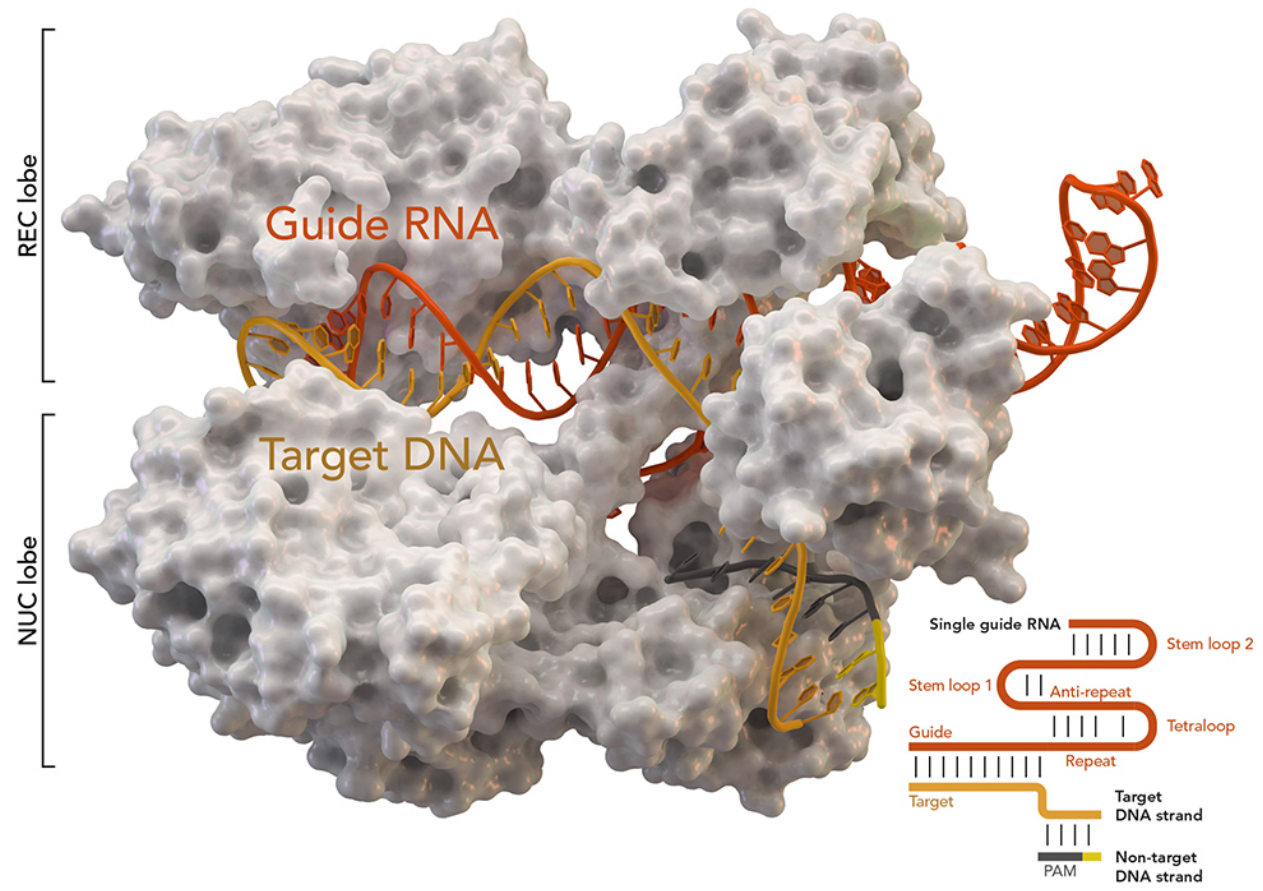

Figure 1.4: Crystalline structure of CRISPR components based on Protein Database ID (PDBID) 5AXW. After recognition of the protospacer-adjacent motif (PAM) sequence (black), the first 20 nucleotide segment of the sgRNA pairs with the unwinding target DNA strand to form an RNADNA heteroduplex, and the non-target DNA strand is displaced inside the Cas9 protein (white). The lobes responsible for binding the sgRNA and DNA — recognition lobe (REC) — and for cleaving the DNA - the nuclease lobe (NUC) are also labeled. Credit: Thomas Splettstoesser (Wikipedia, CC BY-SA 4.0). 
example, gene editing carried out by the clustered, regularly interspaced, short palindromic repeats (CRISPR) associated protein 9 (Cas9) cleaves DNA strands and is directed by a short guide RNA (sgRNA) (see Fig. 1.4) [64-66]. Prediction of the on-target efficiency and off-target activity of CRISPR-Cas9 based on the gRNA sequence is of broad interest in improving gene editing outcomes. Recently, chemical modification of gRNA nucleotides has been shown to enhance the cleavage efficiency of CRISPR-Cas9 [67, 68], so optimal medical outcomes depend on being able to accurately predict the on- and off-target activities with a broad range of possible nucleotide modifications at different positions along the gRNA sequence. Understanding how these modifications affect the RNA function is essential to producing robust, optimal predictions for gene editing. Additionally, recent research has shown that $\mathrm{N}^{6}$-methyladenosine $\left(\mathrm{m}^{6} \mathrm{~A}\right)$ - an abundant and reversible, natural modification in mRNA [69]—enhances long-term memory consolidation (see Fig. 1.3) [53]. Large swaths of literature show the practical medical need for improving our understanding of the epitranscriptome in order to improve human outcomes.

The growing set of known, natural modifications add to the complexity of accurate structure prediction. While determining empirical nearest neighbor energy parameters for the canonical nucleotides has provided secondary structure insights and computational prediction parameters [70], performing this task for all the permutations of the more than 170 known modified nucleotides would be difficult and time consuming. Although a limited number of models support the incorporation of energetic parameters for modified nucleotides[71, 72], no current predictive model completely accounts for the impact of these modifications on RNA structure [73]. While studies have been carried out to computationally model the geometry and base-pairing character of the modified RNA nucleotides [74, 75] and all-atom force fields have been developed for some of the known modifications [76, 
77], few predictive models incorporating modified RNA that are validated by experimental data have been published.

\subsection{Briefly summarizing the main results}

\subsubsection{Project 1: Predicting SHAPE reactivity from 3D structure infor- mation}

To analyze SHAPE reactivity, we used MD simulations to generate conformational ensembles of RNA structures to investigate the correlation between SHAPE reactivity and nucleotide conformational propensity [78]. By combining multiple key factors, such as the nucleotide interaction strength, SHAPE reagent accessibility, and base pairing pattern, we built the 3D structure-SHAPE relationship model (3DSSR), an analytical function that characterizes the conformational flexibility and estimates SHAPE reactivity on the basis of conformational structure information. We showed how 3DSSR may be used to exclude SHAPE-incompatible structures from consideration.

The work led to a publication: Travis Hurst, Xiaojun Xu, Peinan Zhao, and Shi-Jie Chen. "Quantitative Understanding of SHAPE Mechanism from RNA Structure and Dynamics Analysis". In: J. Phys. Chem. B 122.18 (2018), pp. 4771-4783. ISSN: 1520-6106. DOI: 10 .1021/acs .jpcb.8b00575. URL: https:// doi .org/10 .1021/ acs.jpcb. 8b00575. 


\subsubsection{Project 2: Comparing analytical modeling to deep learning ap- proaches for estimating SHAPE reactivity}

Developing a novel convolutional neural network (CNN) model that uses RNA structural data to predict SHAPE reactivity for any given nucleotide, we compared the performance of the CNN model to 3DSSR on RNA molecules that neither model had previously been exposed to. We found that analytical formulations can often provide more insight than pattern recognition methods when limited data is available.

The work led to a publication: Travis Hurst, Yuanzhe Zhou, and Shi-Jie Chen. "Analytical modeling and deep learning approaches to estimating RNA SHAPE reactivity from 3D structure". In: Commun. Inf. Syst. 19.3 (2019), pp. 299-319. DoI: https: / / dx . doi . org/10.4310/CIS.2019.v19.n3.a4. URL: https://WWw.intlpress . com/site/pub/pages/journals/items/cis/content/vols/0019/ 0003/a004/index.php.

\subsubsection{Project 3: Evaluating mechanisms driving reactivity bias and sieving RNA structures with SHAPE}

Reformulating and expanding the 3DSSR model for predicting SHAPE reactivity, we take a direct, physical approach to estimate SHAPE reactivity data as a function of the underlying system energetics [80]. Incorporating 1D sequence information to expand our experimental data set, we discovered sequence-dependent biases in the SHAPE data. Using replica exchange umbrella sampling (REUS) simulations, we found that the stability of unreacted SHAPE reagents in the binding pocket largely explain these sequence-dependent biases. While including correlation effects and noise into the estimator, we reformulated the model to estimate SHAPE data on the basis of the assumption that the SHAPE reactiv- 
ity is a direct reflection of the underlying reaction energetics. This produced much more robust results, with performance improvements of 30\%, 21\%, and $10 \%$ in average Pearson correlation (PC) of the near-native ensemble, PC of the top-ranked structure, and Spearman rank correlation, respectively.

The work led to a publication: Travis Hurst and Shi-Jie Chen. "Sieving RNA 3D Structures with SHAPE and Evaluating Mechanisms Driving Sequence-Dependent Reactivity Bias". In: J. Phys. Chem. B 125.4 (2021), pp. 1156-1166. ISSN: 1520-6106. DOI: 10.1021/acs.jpcb.0c11365. URL: https:// doi .org/10 .1021/acs . jpcb. 0 c11365.

\subsubsection{Project 4: Building an efficient coarse-grained folding force field}

Laying out a cohesive framework for parameterizing a CG force field on the basis of knowledge extracted from a polymer structure database, we used a Bayes-inspired, statistical mechanics approach to construct a CG force field from consecutive simulations and marginal distributions of collective variables extracted from the structure database [81]. Consecutively adding in new marginal distributions, we account for correlations between the collective variables using iterative simulations, providing an estimate of the underlying joint distribution for folding polymers. We further improved the model by showing that parameterization of the force field can be optimized via application of the principle of maximum entropy, which informs the model of the optimal marginal distribution to include in each consecutive parameterization step. Taking this step minimizes the number of collective variables required to parameterize the force field and accelerates the simulations.

The work led to a publication: Travis Hurst, Dong Zhang, Yuanzhe Zhou, and Shi-Jie Chen. "A Bayes-inspired theory for optimally building an efficient coarse-grained folding 
force field". In: Commun. Inf. Syst. 21.1 (2021), pp. 65-83. DOI: https:// dx. doi . org/10.4310/CIS.2021.v21.n1.a4.

\subsubsection{Project 5: Deciphering nucleotide modification-induced struc- ture and stability changes}

To investigate the variable impact of nucleotide modification, we performed alchemical and temperature replica exchange molecular dynamics (TREMD) simulations on an RNA du-

plex and hairpin, respectively [82]. Using a variety of analyses, we used structural information from alchemical simulations to find mechanisms driving modification and mutationdependent stability changes. Using data from the TREMD simulations, we were able to explore a wealth of information related to how modifications alter the energy landscape, depending on their context and type. We also connected these simulations to thermal denaturation data to validate their accuracy.

The work led to a publication: Travis Hurst and Shi-Jie Chen. "Deciphering nucleotide modification-induced structure and stability changes". In: RNA Biol. (2021), pp. 1-11. ISSN: 1547-6286. DOI: $10.1080 / 15476286.2021 .1882179$. URL: https : //doi.org/10.1080/15476286.2021.1882179. 


\section{Chapter 2}

\section{Quantitative Understanding of SHAPE Mechanism from RNA Structure and Dynamics Analysis}

This chapter was published ${ }^{1}$.

Summary: The selective 2'-hydroxyl acylation analyzed by primer extension (SHAPE) method probes RNA local structural and dynamic information at single nucleotide resolution. To gain quantitative insights into the relationship between nucleotide flexibility, RNA $3 D$ structure, and SHAPE reactivity, we develop a 3D Structure-SHAPE Relationship model (3DSSR) to rebuild SHAPE profiles from 3D structures. The model starts from RNA structures and combines nucleotide interaction strength and conformational propensity, ligand (SHAPE reagent) accessibility, and base-pairing pattern through a composite function to quantify the correlation between SHAPE reactivity and nucleotide conforma-

\footnotetext{
${ }^{1}$ Travis Hurst, Xiaojun Xu, Peinan Zhao, and Shi-Jie Chen. "Quantitative Understanding of SHAPE Mechanism from RNA Structure and Dynamics Analysis”. In: J. Phys. Chem. B 122.18 (2018), pp. 47714783. ISSN: 1520-6106. DOI: 10 .1021/acs . jpcb.8b00575. URL: https:// doi.org/10. $1021 /$ acs.jpcb. 8b000575.
} 
tional stability. The 3DSSR model shows the relationship between SHAPE reactivity and RNA structure and energetics.Comparisons between the 3DSSR-predicted SHAPE profile and the experimental SHAPE data show correlation, suggesting that the extracted analytical function may have captured the key factors that determine SHAPE reactivity profile. Furthermore, the theory offers an effective method to sieve RNA 3D models and exclude models that are incompatible with experimental SHAPE data.

\subsection{Introduction}

RNA plays crucial roles in cellular functions at the level of gene expression and regulation. Recent discoveries concerning new functions of non-coding RNAs have led to an unprecedented rise in demand for the determination of RNA 3D structures [1, 2]. However, computational prediction of RNA 3D structures from the sequence remains a significant unsolved problem [1-3]. One of the principal challenges arises from the conformational flexibility, especially in the loop and junction regions, and the resultant rugged energy landscape of RNA. The dynamic structures of RNA lead to the diverse catalytic and regulatory roles of RNA in cellular functions $[5,83]$ such as transcription, mRNA splicing, and translation $[6,7]$. RNA nucleotides contain a ribose sugar on the backbone chain with a base attached to the 1' position of the sugar ring. Phosphodiester backbone rotation facilitates large conformational flexibility of RNA [8]. Hence, base configurations can sample a large portion of the conformational space. RNA bases form a variety of strong base-base and base-backbone interactions [9-11], which can cause a multitude of stable and metastable RNA conformations. The distribution of the collection of heterogeneous conformations determines RNA structure and stability. 
In general, RNA structures contain both rigid and flexible structural elements. The rigid structural elements such as RNA helices and structured loops are primarily stabilized by base-base interactions, such as base pairing and stacking in secondary structures and other noncanonical and long-range interactions in tertiary structures. Both global structural features, such as helix orientations, and local structural features are essential for the interpretation of RNA structural changes in function, such as gene regulation through ligand-induced RNA conformational switches and protein-induced structural changes at active sites.

From an energetics point of view, a flexible RNA can adopt multiple low-energy states from sampling a variety of global folds and local conformations [12-14]. Low-energy state multiplicity results in great difficulties for predicting and modeling RNA 3D structures. For instance, because of the diversity and sequence-sensitivity of loop/junction structures, 3D structure predictions often rely on fragment/template assembly for highly homologous sequences [15-21]. However, given the limited number of known RNA structures, structural motif templates with the required high sequence identity are difficult to attain. As of February 2018, about 3800 structures containing RNA are in the PDB database: approximately 1855 of these are high-resolution crystal structures $(<3 \AA)$. The lack of reliable structural templates for loops and junctions greatly hampers accurate structure prediction.

Inspired by the recent progress in RNA chemical probing methods, researchers have proposed and developed several data-facilitated experimental modeling approaches to complement established template and physics based methods [22-26]. Selective 2'-hydroxyl acylation analyzed by primer extension (SHAPE) is an RNA structure probing technology that provides significant insights into local nucleotide structure and dynamics [27, 28]. SHAPE reagents are small ligands: 1-methyl-7-nitroisatoic anhydride (1M7), 1-methyl-6nitroisatoic anhydride (1M6), N-methylisotoic anhydride (NMIA), benzoyl cyanide (BzCN), 
2-methyl-3-furoic acid imidazolide (FAI), and 2-methylnicotinic acid imidazolide (NAI) that form a 2'-O-adduct with the nucleotide 2'-hydroxyl group [29]. Previous studies [3032] suggested that unconstrained nucleotides that sample more flexible conformations have greater probability to adopt the SHAPE-reactive conformations and, thus, show higher SHAPE reactivity. In contrast, nucleotides that are constrained by base pairing and stacking interactions are much less reactive. This quantitative measurement of local nucleotide dynamics makes SHAPE an effective tool to probe whether a nucleotide forms a base pair (either in a helix or in a structured loop) or remains unpaired in a flexible loop/junction. In comparison to modeling without experimental data, SHAPE data places effective constraints on RNA conformational space and can significantly improve the efficiency of conformational sampling. SHAPE-directed RNA structure prediction substantially increases the accuracy of RNA secondary structure modeling [33-37], where SHAPE reactivities that signal nucleotide dynamics provides additional structural constraints for the free-energy based predictions [38]. Moreover, combined with other strategies, such as differential SHAPE reactivity, mutate-and-map, and time-resolved SHAPE chemistry, SHAPE probing provides highly useful information for the in vitro and in vivo determination of noncanonical tertiary interactions and RNA kinetics[39-45].

Although SHAPE technology is widely applied and is a highly valuable tool to RNA modeling and (2D) structure prediction, the mechanism of the SHAPE reaction is not fully understood. A variety of conformations render a nucleotide reactive by SHAPE [33, 34]. Specific RNA structural features correspond to high SHAPE reactivity, including a long PO-to-2'-OH distance, short 2'-OH to $\mathrm{O} 2 / \mathrm{N} 3$ distance, and short 2'-OH to non-bridging $\mathrm{O}$ distance. These conclusions [32] were mainly derived from SHAPE mapping data for 16S rRNA in ribosome crystals and focused on the conformations that tend to increase SHAPE 
reactivity. Low SHAPE reactivities are known to correlate with positions in helices and rigidly structured loops, but the full mechanisms by which nucleotides can be conformationally constrained, and rendered inert, are not fully understood. Additionally, the accuracy of SHAPE experiments relies on carefully chosen experimental and data processing methods $[35,37]$. While SHAPE data may suggest that an unreactive nucleotide forms an interaction with another member of the system, SHAPE cannot give us information about who is interacting with the nucleotide or what kind of interaction is formed[35]. These limitations mean that SHAPE-directed structures should not be considered absolutely determined, but SHAPE can give us useful structural hypotheses[35]. Although SHAPE has limitations, prediction of 2D structure using SHAPE has reached a level of accuracy on par with comparative sequence analysis [33]. Conversely, by calculating the effective stability determined by O2'-P-O5' angle fluctuations and optimizing the native structure-based potential using MD simulations, SHAPE profiles have been rebuilt [46]. In this study, we focused on developing ananalytical function for quantitative prediction of the SHAPE profile from individual RNA 3D structures, and applying our function to exclude SHAPEincompatible structures.

To analyze SHAPE reactivity, we used conformational ensembles generated by MD simulations to investigate the correlation between the SHAPE reactivity and the nucleotide conformational propensity. Then, by combining multiple key factors, such as the nucleotide interaction strength, SHAPE reagent accessibility, and base-pairing pattern, we built an analytical function - the three-Dimensional Structure-SHAPE Relationship (3DSSR) function-to characterize the conformational flexibility and SHAPE reactivity based on the conformational and energetics information. Finally, we showed how the 3DSSR function can be used to exclude some 3D structures based on experimental SHAPE data. 


\subsection{Methods}

\subsubsection{Interaction intensity for base pairs and base stacks}

Each base pairing interaction between two RNA nucleotides can be divided into 12 families based on the geometric conformations involving three nucleotide edges: Watson-Crick, Hoogsteen, and Sugar [9]. Based on statistical frequencies derived from 41 RNA structures in the PDB database [9], we assigned pseudo-free energy potentials to each base pair interaction class according to the quasi-chemical approximation [84]:

$$
E_{\mathrm{bp}}^{(t)}(i, j)=-k_{B} T \ln \left(N_{\mathrm{obs}}^{(t)}(i, j) / N_{\exp }^{(t)}(i, j)\right)
$$

for type- $t$ base pair $(t=1,2, \cdots, 12)$. Here, $i$ and $j$ refer to the base types (A,U,G,C), and $N_{\text {obs }}^{(t)}(i, j)$ and $N_{\exp }^{(t)}(i, j)$ are the observed number and the expected number, respectively, of type- $t$ base pair $(i, j)$ in the database. $N_{\exp }^{(t)}(i, j)$ is computed as

$$
N_{\exp }^{(t)}(i, j)=N_{\mathrm{obs}}^{(t)} x_{i} x_{j}
$$

where $N_{\mathrm{obs}}^{(t)}$ is the total observed number of type- $t$ base pair and $x_{i}$ and $x_{j}$ are the mole fractions of the nucleotide types $i$ and $j$ in the entire database, respectively. The database for the 12 types of RNA base-pairing families contained 3917 entries [9]. The canonical cis Watson-Crick/Watson-Crick conformation (abbreviated as WWc) occurs much more frequently (762 for WWc C-G, 771 for WWc G-C, 205 for WWc A-U and 238 for WWc U-A) than the occurrences (mostly less than 50) of the other non-canonical base pairs. We identified the base pair types with RNAview software [9]. 
The above interaction intensities are intended to be used as a (crude) measure for the relative depths of the potentials of mean forces for the different base pairs. These parameters are not real physical free energies, which are determined by the Boltzmann-averaged (nonadditive) energies for the whole structure. Therefore, rigorously speaking, due to the correlation between the different interactions, the sum of the interaction intensities for all the nucleotides does not add up to the total interaction energy or free energy of the system. However, given the Turner parameters for the different types of base stacks in a helix, the above pseudo-energy parameters may provide a crude estimate for the relative interaction strengths of the different noncanonical base pairs. For a loop, the different interactions may result in different nucleotide flexibilities. In contrast, for a helix, the structure is relatively rigid, so the different interaction strengths of the base pairs would not cause notable differences in nucleotide flexibility. Accounting for this, we suppressed the effect of the pseudo-energy parameters for helices in our model (see factor $B P(n)$ in Eq. 2.7 below).

To quantify the energy of the stacked base conformation, we measured the distance and plane angle between the bases for each pair of stacked nucleotides. The base plane is defined as the triad of $\mathrm{C} 2, \mathrm{C} 4$, and C6 atoms. The center of each base is set as the center of the C2-C4-C6 triad. For nucleotides to meet the base-stacking criteria, the distance between the centers of the bases must be less than $9.5 \AA$, and the base angle must be less than $30^{\circ}$.

To account for the effect of nucleotide interaction strength, we propose the following interaction function to characterize the interaction intensity for nucleotide $n$ :

$$
I I(n)=\sum_{m}\left[a \cdot E_{\mathrm{bp}}^{(t)}(n, m)+b\right]+\sum_{m} E_{s t}(n, m) .
$$

The terms on the right hand side account for the base pairing and stacking interactions 
between $n$ and its interacting partner $m$. The base pairing interaction $E_{\mathrm{bp}}^{(t)}(n, m)$ between nucleotide $n$ and $m$ is sequence and base pairing type, $t$, dependent. Because helix stacking is accounted for in the $B P(n)$ term (see Eq. 2.7 below), the base stacking interaction energy, $E_{s t}$, for a pair of stacked bases, $n$ and $m$, primarily considers loop stacking and is assumed to be sequence independent. Consecutive base pairs tend to form base stacks, so base pairing and base stacking can be coupled. The prefactor $a$ introduced in Eq. 2.3 is a weighting factor to account for the correlation between base pairing and base stacking, while $b$ is the minimum energy for base-pairing.

\subsubsection{Ligand accessibility calculation}

The ligand (SHAPE reagent) accessibility is another important factor to the SHAPE reaction. If a nucleotide 2'-OH is buried inside the RNA structure, SHAPE reagents cannot react with that site, which reduces the SHAPE reactivity. Ligand accessibility is expected to be a necessary condition for SHAPE to react.

The free SHAPE ligand, usually 1M7 or NMIA, has an effective radius between 2.0 and $2.5 \AA$ (see Fig. A.1 in Appendix A for more details). The accessible surface of 2'$\mathrm{OH}$ is calculated using VMD [85]. The overall ligand accessibility result is found to be insensitive to different probe sizes within this range because different probe sizes generally induce insignificant changes in the ligand accessible surface area.

\subsubsection{All-atom molecular dynamics sampling of near-native structure}

Starting from the experimentally determined 3D structure, we estimated the nucleotide flexibility using all-atom molecular dynamics (MD) simulations. The MD simulations were 
carried out using the NAMD 2.10 package [86] with CHARMM27 and Amber16 [87] with the Amber force fields for nucleic acids to verify that our results were force field independent. The methods and results presented here are based on Amber16, but similar results were also obtained using NAMD. The RNA molecule was embedded in a TIP3P[88] truncated octahedral water box with a water shell of $15 \AA$. Sodium and chloride ions were used as counterions in order to neutralize the RNA molecule and to keep 1M sodium concentration of the system. Sodium ions were manually added according to

$$
N_{N a}=6.022 * 10^{-4} * V
$$

where the number of sodium ions $\left(N_{N a}\right)$ depends on the volume in $\AA^{3}(V)$ automatically output by tleap upon creation of the water box. Subsequently, chloride ions were added to neutralize the overall charge. The temperature was kept constant at $300 \mathrm{~K}$ by coupling the system to a Langevin heat bath. During the MD simulations, the backbone phosphate (P) atom positions and two nucleotide atom positions in base pairing atoms (C2, C6) were strongly constrained with a restraint weight of $500 \mathrm{kcal} * \AA^{2} /$ mol to maintain the global folding and helical base pairing structure of each RNA molecule. Using the leaprc.ff99bsc0 force field, we sampled the conformations of each nucleotide under the structural constraints such as base pairing and stacking. We used four simulation steps. The first step held the RNA fixed and minimized the energy of the surrounding ions and water. The second step minimized the entire system energy. The third step warmed the system to $300 \mathrm{~K}$. The fourth step was the production step. With the crystalline PDB structure as the initial state for each RNA in the training set, the coordinates of all the atoms were written to the NAMD DCD (Amber MDCRD) file every 5 ps, which yielded 1000 snapshots of each case over 5 ns. 


\section{Training the parameters in the 3DSSR function}

We constrained the backbone and base paired nucleotides during the MD simulations for each of our test cases, so the nucleotides were constrained to remain near their crystalline position. Dynamical analysis with relaxed constraints showed poor agreement with experimental SHAPE data, so we chose constraints that held the global crystal structure but allowed loop nucleotides to sample local conformational space. For each of our 12 cases, we assumed that each of the 1000 near-native structures could feasibly represent the native structure found in a solution environment, which is consistent with how SHAPE experiments are performed. Thus, we were able to grow our training set a thousand-fold. The parameters $a, b, E_{s t}$, and $S_{0}$ in the 3DSSR function of Eq. 2.7 were optimized by randomly choosing these values, finding the average correlation of all thousand near-native MD generations for each case, and maximizing the average correlations for all of the cases.

To cross validate, the parameters were also optimized using the leave-one-out procedure. For the 12 RNA models we tested (see "RNA structures used for validation" section below), we defined each set of 1000 solution-native MD structures as the test set and obtained twelve training sets consisting of all the other $(11,000)$ MD structures. We optimized the parameters by maximizing the average Pearson correlation coefficient between the experimental SHAPE data and our predicted SHAPE profiles in each training set.

To further test our parameters, we trained using a genetic algorithm, wherein we calculated the correlations for a population of 100 sets of parameters. For each iteration, the top 10 percent of parameters were maintained and the bottom 90 percent were mated using

evolutionary principals. Then, we ranked the sets of parameters based on their respective correlations. 


\section{Off-native 3D sampling using coarse grained simulations}

To test the ability of our algorithm to discriminate between near-native and non-native 3D structures, we sampled non-native 3D structures using a newly developed coarse grained (CG) model [53]. We tested our ability to exclude non-native 3D structures by using both the native 2D structure and non-native 2D structures that were generated by Mfold [89] (see Tables A.3-A.6 in Appendix A). The non-native 2D structures were often very similar to their native counterparts, so this test allowed us to establish that 3DSSR meets a minimum secondary structure sensitivity benchmark.

Starting decoy 3D structures were generated from the non-native 2D structures using Vfold3D [90]. Maintaining the initial 2D structure, the CG simulations were performed. To enhance conformational sampling, we used Replica-Exchange MD (REMD) with 8 replicas for temperatures from 125 to $300 \mathrm{~K}$. The total simulation time per replica was set to $t=5 \mathrm{~ns}$ with integration time-step $\Delta t=0.5$ fs. For each case, we collected about 7400 snapshots from these simulations with all heavy-atom, global RMSD from the native structure ranging from 0.5 to $14-40 \AA$ depending on the size of the RNA.

\section{Interaction Network Fidelity value calculation}

To distinguish between SHAPE-compatible and SHAPE-incompatible structures, we utilized the Interaction Network Fidelity (INF) value to quantify the similarity of interaction pattern between decoy structures and near-native structures. The INF is calculated as

$$
\mathrm{INF}=\sqrt{\left(\frac{T P}{T P+F P}\right) \times\left(\frac{T P}{T P+F N}\right)},
$$

where TP is the number of correctly predicted base-base interactions (BBI), FP is the 
number of predicted BBI in the test model with no corresponding interaction in the accepted model, and FN is the number of $\mathrm{BBI}$ in the native model not present in the test model. To find these interactions, we utilized the RNAview [9] and the stacking calculations mentioned earlier.

The INF value is more useful than the global RMSD in distinguishing between these structures because SHAPE reactivity is determined by the ability of individual nucleotides to sample a variety of configurations. While two structures may have relatively high RMSD between them, the INF between these structures may be similar, and our predicted SHAPE profile may also be similar. In addition, two structures may have low RMSD from the native structure but have very different predicted SHAPE profiles, whereas if the INF for a structure is high, the correlation of its predicted profile with experimental SHAPE data should also be high.

\section{RNA structures used for validation.}

We analyzed the SHAPE profiles for twelve RNAs: the (174-nt) lysine riboswitch from T. maritima, the (79-nt) TPP riboswitch from E. coli, the (71-nt) adenine riboswitch from $V$. vulnificus, the (117-nt) SAM-I riboswitch from B. subtilis, the (154-nt) specificity domain of Ribonuclease P RNA, the (93-nt) cyclic-di-GMP riboswitch from V. cholera, the (29-nt) TAR RNA from HIV-1, the (30-nt) U1A protein binding site RNA from H. sapiens, the (76nt) aspartate tRNA from Yeast, the (75-nt) preQ1 riboswitch aptamer from B. subtilis, the (36-nt) M-box riboswitch from B. subtilis, and the (154-nt) M-box riboswitch from B. subtilis . The crystallographic PDB IDs are 3DIG, 2GDI, 1Y26, 4KQY, 1NBS, 3IWN, 2L8H, 1AUD, 1EHZ, 1VTQ, 2L1V, and 3PDR, respectively. For RNA structures with multiple PDB entries in the database, we chose the one with the highest resolution. The SHAPE pro- 
files for these RNAs are from the published experimental data [30, 36, 37] (see Table A.2 in Appendix A for the experimental conditions).

\subsection{Results}

The inter-nucleotide contacts of the folded 3D structure determine the local nucleotide dynamics of an RNA. For example, the nucleotides in helices, which involve strong base-base pairing and stacking interactions, can have only small fluctuations around their equilibrium positions, while nucleotides that do not interact with other nucleotides usually have larger fluctuations. Therefore, local nucleotide dynamics is correlated with the interaction strength as well as the 3D structure. Information from experiments [23-26], such as SHAPE[39-41], relating structure and dynamics can facilitate our understanding of the interaction energetics and structural features in RNA.

\section{RNA 3D structure and SHAPE reactivity}

There is a strong general tendency for flexible RNA nucleotides to be SHAPE-reactive. Based on SHAPE discrimination between helix and loop regions, SHAPE-assisted RNA secondary structure prediction algorithms have been developed and have led to many successful applications [33-37]. However, the relationship between RNA secondary structure and SHAPE reactivity can be complex, in significant part because not all loops and junctions are conformationally flexible. For example, some loop regions consistently show low SHAPE reactivities [33-37, 91]. The nucleotides in such loops usually engage in constraining (non-canonical) interactions including irregular base pairs, base stacking, base triplets, 
and other higher-order interactions.
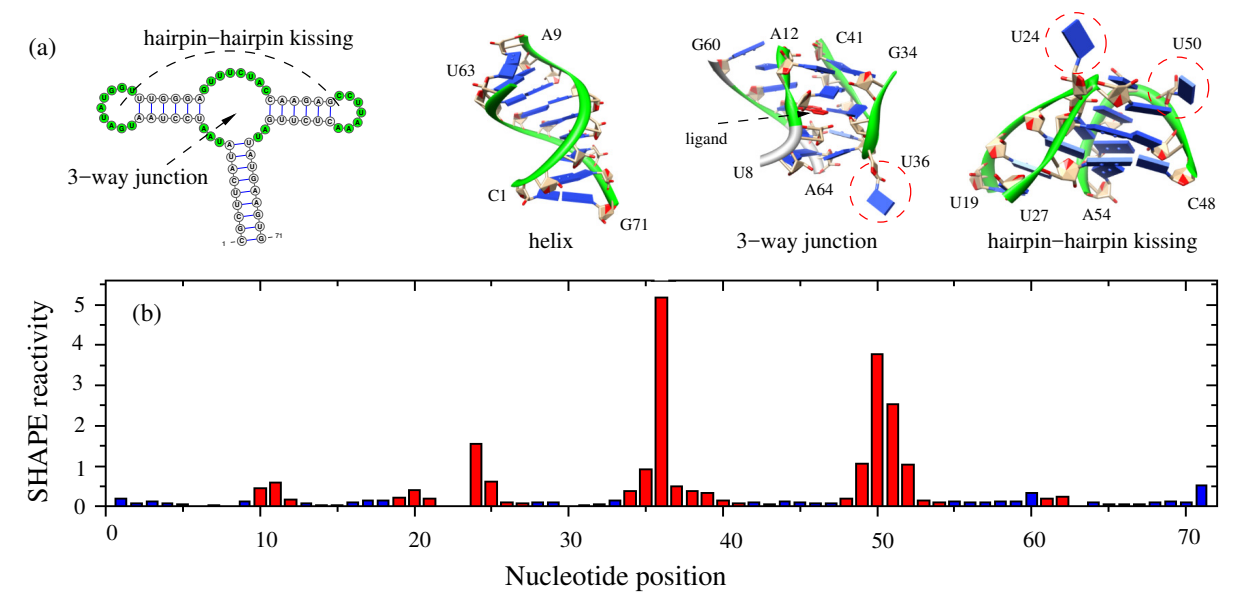

Figure 2.1: (a) The secondary structure of the Adenine riboswitch (PDB: 1Y26), the 3D structures of the helix, the 3-way junction and the hairpin-hairpin kissing motif. (b) Histogram of SHAPE reactivity [37] as a function of nucleotide position for 1Y26. The blue and red bars are for the nucleotides in helices and in loops/junctions, respectively. Not all the nucleotides in loops/junctions have high SHAPE reactivity, due to the constraints by other nucleotides and/or other molecules.

As shown in Fig. 2.1 for the Adenine riboswitch (PDB: 1Y26), nucleotides with high SHAPE reactivity come from flexible loops, and no significant interactions are involved because these nucleotides have their bases pointing to solvent. For example, nucleotide U36-a 3-way junction nucleotide- and nucleotides U24 and U50-hairpin-hairpin kissing motif nucleotides - display very high SHAPE reactivity because they engage in no significant interactions with other nucleotides. However, when compared to SHAPE values for nucleotides within helix regions, not all nucleotides in loop regions, colored red, have high SHAPE reactivity. In most cases, the low SHAPE reactivity in these loop regions can be explained by non-canonical, constraining interactions. While SHAPE-reactivity in loops depends on the flexibility of the specific loop, almost all the nucleotides in helix regions, colored blue, exhibit low SHAPE reactivity. Therefore, for nucleotides with low SHAPE reactivity, discerning whether nucleotides are in highly structured loops or rigid helices is 
a challenge. SHAPE probing for a nucleotide cannot provide details about what specific interactions are involved. On the other hand, for a given RNA 3D structure, all the existing interactions may provide significant information about the rigidity/flexibility of each nucleotide and convey to us the ability to predict the SHAPE profile for a given 3D structure.

\section{SHAPE reactivity vs. nucleotide flexibility}

We characterized the nucleotide flexibility using conformational fluctuations in MD simulations. As shown in Figs. 2.2bd, nucleotides within the helices have very small fluctuations due to the strong constraints of canonical base pairing and base stacking. However, nucleotides in loop/junction regions may undergo large fluctuations due to weak interactions from other nucleotides. As highlighted in Fig. 2.2d, for example, nucleotides U24, U36, and U50 sample more conformations than other nucleotides since their bases point outward into the solvent, making them prone to large conformational fluctuations around the equilibrium states. However, not all nucleotides in loops/junctions have large fluctuations since some may engage in non-canonical base pairing and other high-order noncanonical interactions.

To depict the correspondence between SHAPE and nucleotide flexibility, we extracted the sampled conformations from MD trajectories and calculated the pairwise root-meansquare deviation (RMSD) for each pair of nucleotide conformations. The MD-estimated flexibility for each nucleotide $(i)$ is defined as the average of the pairwise structural distance $\mathrm{RMSD}_{p}$ for the nucleotide $i$ :

$$
\operatorname{RMSD}(i)=\frac{1}{N} \sum_{p=1}^{N} \operatorname{RMSD}_{p}(i)
$$




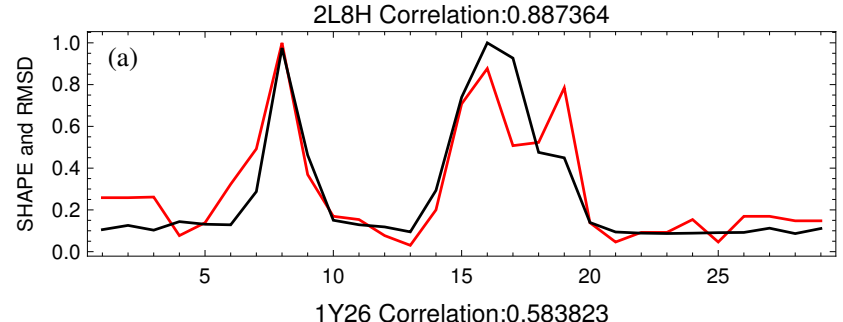

(d) 1 Y 26
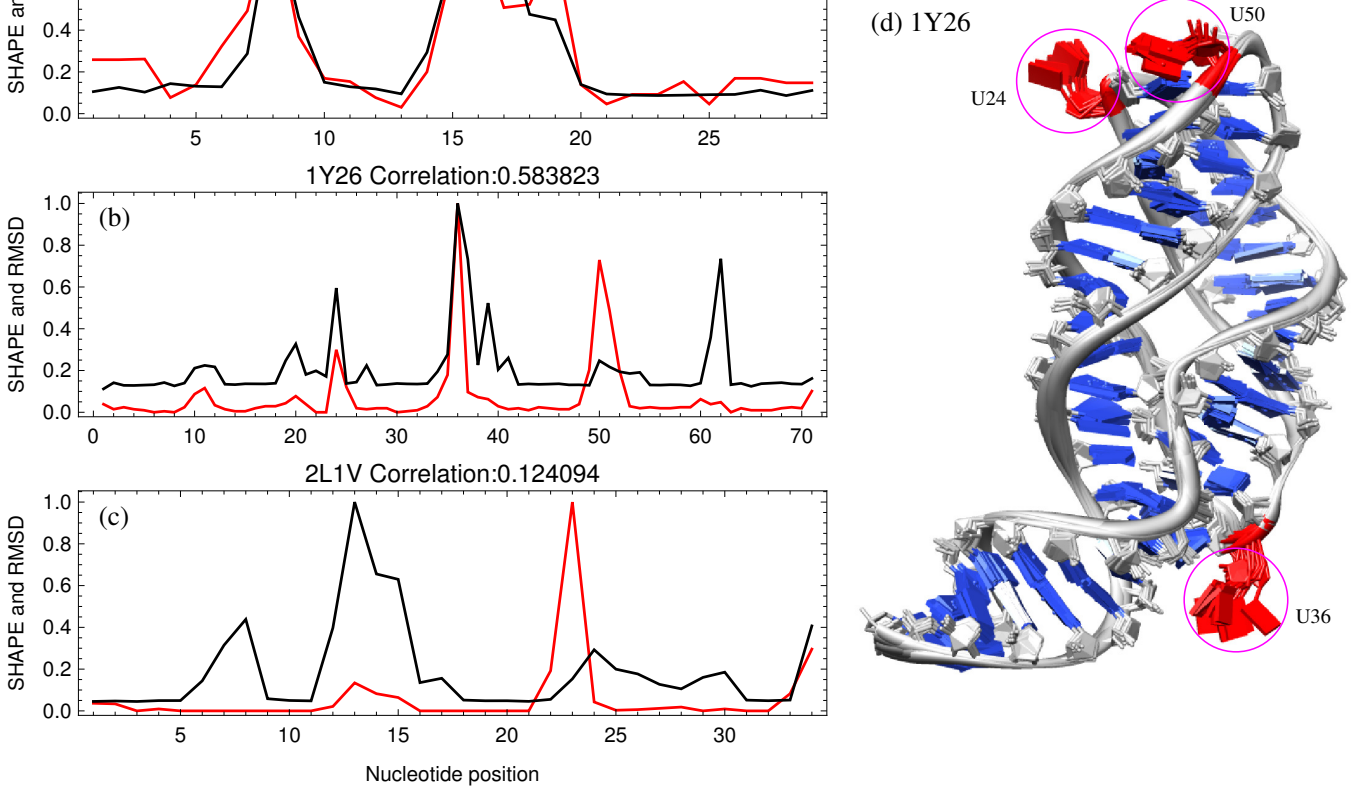

Figure 2.2: Experimental SHAPE data is generally correlated with nucleotide dynamics. The scaled SHAPE reactivities [30, 36, 37] (shown in red) as a function of nucleotide position compared with the MD-estimated nucleotide flexibilities (scaled RMSD shown in black) for the highest correlated case (a) $2 \mathrm{~L} 8 \mathrm{H}$, the example case (b) $1 \mathrm{Y} 26$, and the lowest correlated case (c) $2 \mathrm{~L} 1 \mathrm{~V}$, respectively. (d) Ten snapshots of an MD simulation for 1Y26, with backbone phosphate (P) atoms position-constrained. The global fold of the RNA molecule is conserved, while each nucleotide has different fluctuations around its equilibrium state due to the constraints provided by other nucleotides. Highlighted in red are three nucleotides (U24, U36 and U50) that have large fluctuations and the highest SHAPE reactivity. The ligand molecules are not included during MD simulations. 
Here, the summation is over all the possible $(N)$ pairs of conformations. Because we strongly constrained the global structure, the RMSD for each nucleotide could be independently calculated.

For each case, we compared SHAPE reactivity with nucleotide RMSD (Figs.2.2abc and Fig. A.3 in Appendix A). For visualization purposes, the SHAPE reactivities and RMSD (see Eq. 2.6) values for each case shown in Fig. 2.2 have been scaled by their respective maximum values to range from 0 to 1 . For reference, the RMSDs for nucleotides in helices are around $0.25 \AA$, while the RMSDs for nucleotides within loops/junctions depend on the constraining interactions with other nucleotides. The comparison shows positive correlation as established by the average Pearson correlation coefficient of 0.51 for all cases. An exception to this is the smaller M-box riboswitch from B. subtilis (2L1V), whose dynamic correlation to SHAPE was low (Fig. 2.2c). However, the ligand accessibility was especially important for this compact structure as seen in Table 2.1, and multiple MD simulations may help attenuate overestimated parts of the dynamic profile and improve the correlation to SHAPE.

The RMSDs for nucleotides U24, U36, and U50 highlighted in Fig.2.2d, are 1.05, 1.77, and $0.44 \AA$, respectively. This indicates that SHAPE chemistry can reflect local structure fluctuation at single nucleotide resolution. While SHAPE chemistry is also likely to be influenced by dynamics on the timescale of seconds or longer, the results here, based on nano-second motions, suggest that the SHAPE reaction might also be sensitive to relative, local nucleotide dynamics on timescales much shorter than seconds[30]. However, to more thoroughly sample the available conformational space, combining multiple simulations may be helpful to account for the full SHAPE profile. 
Table 2.1: Pearson correlations between the experimental SHAPE data and the 3DSSRpredicted SHAPE profiles using the different combinations of the three factors: interaction intensity $(I I)$, base pairing pattern $(B P)$, and ligand accessible surface (sas $=S A S+S_{0}$ ). Although the correlations generally decrease as sequences get longer, the log-scaled pvalues, representing the statistical significance of our results, are displayed to show that our method retains significance.

\begin{tabular}{l|c|c|c|c|c|c|c|c|c}
\hline \hline PDB & \multirow{2}{*}{ \# nt } & $\frac{1}{|I I-1|}$ & $B P$ & sas & $\frac{B P}{|I I-1|}$ & $B P \cdot$ sas & $\frac{\text { sas }}{|I I-1|}$ & Pred. & \multirow{2}{*}{$\log (\mathrm{p})$} \\
\hline 2L8H & 29 & 0.83 & 0.82 & 0.10 & 0.89 & 0.93 & 0.88 & 0.96 & -16 \\
1AUD & 30 & 0.83 & 0.75 & -0.04 & 0.90 & 0.83 & 0.83 & 0.92 & -13 \\
2L1V & 36 & 0.49 & 0.20 & 0.56 & 0.51 & 0.53 & 0.92 & 0.83 & -10 \\
1Y26 & 71 & 0.78 & 0.38 & 0.26 & 0.76 & 0.60 & 0.88 & 0.88 & -24 \\
1VTQ & 75 & 0.57 & 0.51 & 0.18 & 0.68 & 0.59 & 0.67 & 0.71 & -12 \\
1EHZ & 76 & 0.70 & 0.46 & 0.40 & 0.69 & 0.65 & 0.80 & 0.80 & -18 \\
2GDI & 79 & 0.84 & 0.53 & 0.43 & 0.80 & 0.75 & 0.88 & 0.89 & -28 \\
3IWN & 93 & 0.67 & 0.53 & 0.32 & 0.73 & 0.67 & 0.68 & 0.74 & -17 \\
4KQY & 117 & 0.64 & 0.54 & 0.17 & 0.69 & 0.65 & 0.67 & 0.75 & -22 \\
1NBS & 154 & 0.49 & 0.46 & 0.18 & 0.57 & 0.57 & 0.52 & 0.61 & -16 \\
3PDR & 154 & 0.50 & 0.31 & 0.16 & 0.59 & 0.46 & 0.61 & 0.61 & -18 \\
3DIG & 174 & 0.57 & 0.43 & 0.21 & 0.58 & 0.59 & 0.66 & 0.70 & -26 \\
\hline Ave. & 92 & 0.66 & 0.49 & 0.24 & 0.70 & 0.65 & 0.75 & 0.78 & -18 \\
\hline \hline
\end{tabular}

\subsubsection{The three-Dimensional Structure-SHAPE Relationship (3DSSR) function: Prediction of SHAPE profiles from 3D structure and energetics}

The above results show the positive correlation between SHAPE reactivity and the MDestimated nucleotide flexibility. However, it is computationally inefficient to estimate nucleotide flexibilities for RNAs by MD simulations [92], especially for large RNAs. Therefore, we propose an analytical function, namely the "3D Structure-SHAPE Relationship" (3DSSR) function:

$$
P(n)=B P(n) \cdot \frac{S A S(n)+S_{0}}{|I I(n)-1.0|}
$$


to estimate the nucleotide stability. Here, $P(n)$ is the predicted SHAPE reactivity of the nucleotide $\mathrm{n} ; I I(n)$ and $S A S(n)$ are the aforementioned interaction intensity value (see Eq. 2.3) and ligand accessible 2'-OH surface area of the nucleotide n, respectively. $S_{0}$ is a constant, accounting for the dynamics of each nucleotide in solution during SHAPE probing and the possibility of a nucleotide becoming solvent accessible during the experiment.

Unlike $I I(n)$, which represents the effect of interaction, $B P(n)$, the base-pairing factor, represents the effect of structure. Specifically, $B P(n)$ is assigned a lower constant value of 0.01 for nucleotides in helix regions and a higher constant value of 1.0 for nucleotides in the flexible (loop and junction) regions. The values assigned in helix regions are much lower, which guarantees the SHAPE-profile prediction will be low. The relatively high values assigned to loops and junctions are modulated by the other factors to differentiate between flexible loops and rigid loops. According to our calculation, modifying the constant value in the denominator of $P(n)$ (0.01 or 1.0) makes little difference to the final result, as it simply sets the upper limit on the score we assign to the nucleotide. Furthermore, the small value of $B P(n)$ assigned to the nucleotides in helices silences the effect of the interaction intensity for the different base pairs/stacks. Consistent with the SHAPE experimental data, predicted SHAPE values for helix nucleotides have little variation.

The ligand accessibility value describes the probability that a given nucleotide is exposed to the SHAPE reagent. The base-pairing pattern distinguishes helix and loop regions. The interaction intensity value describes the local rigidity of the nucleotide and can distinguish rigid nucleotides from flexible nucleotides in loop regions. As predicted by the above 3DSSR function, any helix nucleotides with low ligand accessibility would be highly unlikely to reach a high SHAPE value. For the ligand accessible loop or junction nucleotides, the interaction intensity can modulate the predicted SHAPE value to describe 
its local flexibility and distinguish rigid and flexible loops. Since the information about local nucleotide stability and dynamics is embedded in the 3D structure and energetics, the 3DSSR function, combined with the effects of the interaction intensity $(I I(n))$, ligand accessibility of 2'-OH $(S A S(n))$, and base pairing pattern $(B P(n))$, accounts for both the structural environment and the interaction energetics of the nucleotides.

\section{Training the parameters}

As shown in Table A.1 in Appendix A, the parameters that optimize the total correlation in the leave-one-out random search method are consistent for all twelve training sets. Additionally, the average parameters of the twelve training sets are consistent with the parameters found using random search training and the genetic algorithm for all structures. The consistency of the parameters for the different RNAs under different experimental conditions suggest that the above 3DSSR function might not be very sensitive to the solution conditions, such as ion concentrations, provided that the RNA can fold into the stable native structure (see also Table A.2 in Appendix A for the experimental conditions). However, it is important to note that for a given native structure, the solution condition can influence the global folding stability.

Using the parameters that maximize the correlation for all of the MD cases, we found an average correlation of 0.78 for the best cases. Because we found good agreement between our different training methods, we finally set the values of parameters as the optimal parameters that maximize the average correlation for all MD cases: $a=0.03, b=-0.49$ $\mathrm{kcal} / \mathrm{mol}, E_{s t}=-0.24 \mathrm{kcal} / \mathrm{mol}$, and $S_{0}=15 \AA^{2}$.

As listed in Table 2.1, the correlation between the experimentally determined SHAPE data and the 3DSSR-predicted SHAPE profile ranges from 0.61 to 0.96 and averages 0.78 . As shown in Fig. 2.3, the 3DSSR function predicts most high SHAPE peaks, even for 

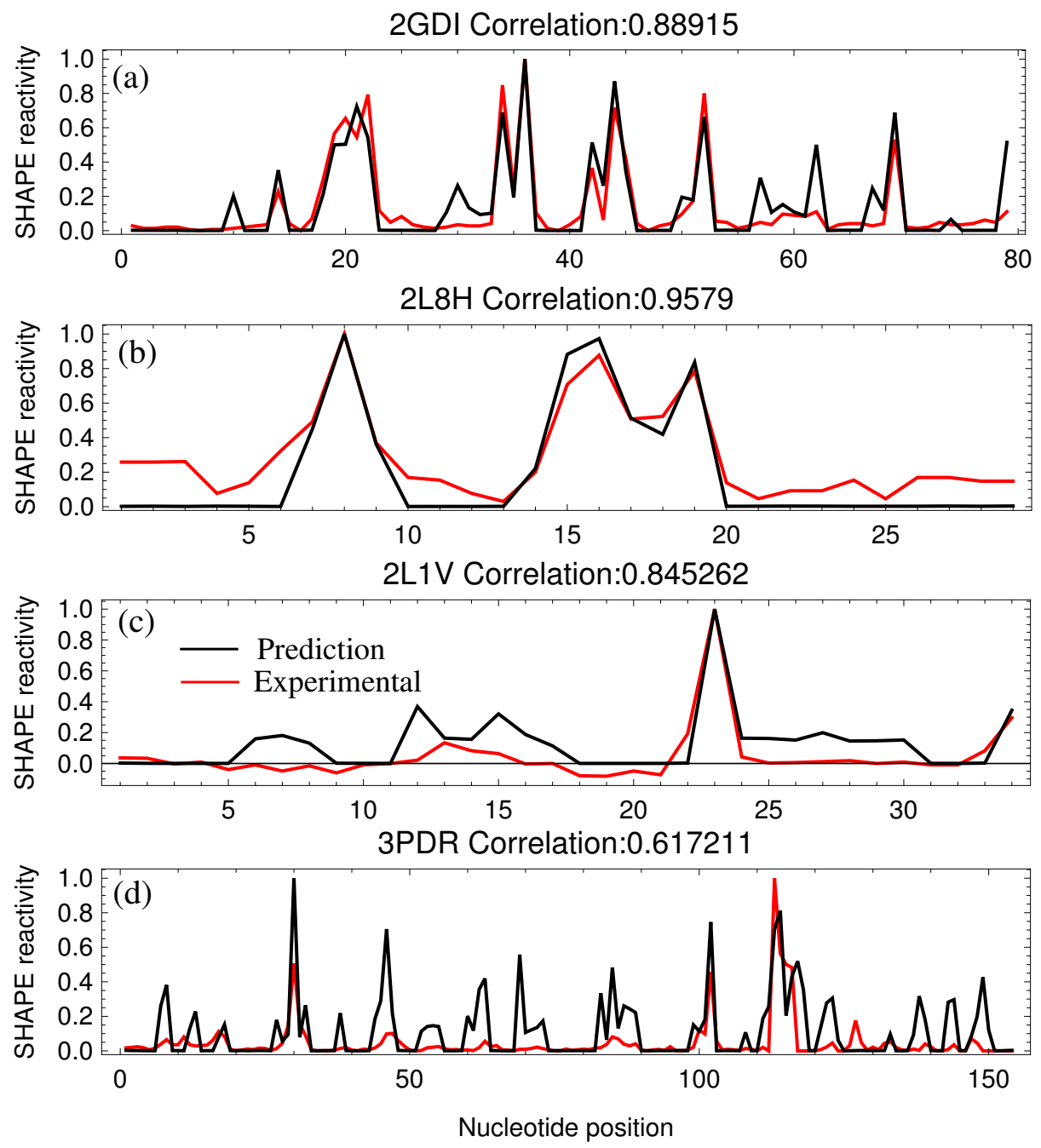

Figure 2.3: (a) Comparison between the predicted (black) and the experimental (red) SHAPE profiles for the TPP riboswitch (2GDI) [36], the case with the smallest p-value. (b) Comparison of SHAPE profiles for the TAR RNA (2L8H) [30], the case with the highest correlation. (c) Comparison of SHAPE profiles for the (small) M-Box Riboswitch (2L1V) [36], the case with the largest p-value. (d) Comparison of SHAPE profiles for the (large) M-Box Riboswitch (3PDR) [36], the case with the lowest correlation. 
the worst predictions, 3PDR and 2L1V. As expected, for the low SHAPE reactivity of the helix nucleotides, 3DSSR shows good agreement with the experimental data. However, for nucleotides within structured loops/junctions of low SHAPE reactivity, the interaction intensity $I I(n)$ combined with the ligand accessibility $S A S(n)$ can distinguish a rigid, structured-loop nucleotide from a flexible, unstructured-loop nucleotide of high SHAPE reactivity. While the 3DSSR function can discriminate most unstructured nucleotides from structured ones, the 3DSSR cannot predict the exact SHAPE reactivity profile for each nucleotide without specific information about SHAPE-reactive and inert conformations. Therefore, direct comparison for the absolute SHAPE reactivity values is not possible with our model. Because of this limitation, using the Pearson correlation coefficient to compare the predicted SHAPE profile (shape of the curve) to the experimental SHAPE profile provides a more effective test for the theory.

\section{Testing the 3DSSR function}

Our tests consistently show correlation between the 3DSSR function and the experimental SHAPE data for each of the 12 RNA molecules, as listed in Table 2.1. The SHAPE reactivity function contains three parts: interaction intensity, ligand accessibility, and base pairing pattern. As listed in Table 2.1, no individual component of the 3DSSR function is as highly correlated with the experimental SHAPE profile as the 3DSSR function taken in its entirety, which indicates that the mechanism of SHAPE depends on multiple factors. The average correlations of individual components in 3DSSR to SHAPE data are 0.66, 0.49 , and 0.24 for interaction intensity, base pairing pattern, and ligand accessibility, respectively. Therefore, the interaction intensity makes the major contribution to the SHAPE pattern. The base pairing pattern (secondary structure) has the second largest correlation 
to SHAPE data. $B P(n)$ alone can reach 0.49 average correlation, which is why SHAPE technology can be used to improve the accuracy of RNA secondary structure prediction. Although the SHAPE reagent molecule directly binds to the 2'-OH on the nucleotides, the ligand accessible surface of 2'-OH group of each nucleotide alone shows the weakest correlation, which is consistent with the previous study [30].

Furthermore, the combinations of any two factors (shown in Table 2.1) show improvements over individual factors but failed to show better correlation than the complete function. These calculations again show that interaction intensity is the main contribution to SHAPE reactivity. Nucleotides with weaker and fewer interactions generally have a greater ability to sample SHAPE-reactive conformations. However, the modulation of this component is necessary to optimize the algorithm. The predicted profile successfully rebuilt the majority of SHAPE peaks (Fig. 2.3 and Fig. A.2 in Appendix A). The correlation of the 3DSSR function is better than the correlation of any isolated 3DSSR function component.

\section{Excluding SHAPE-incompatible 3D structures}

To support the idea that we can use our algorithm to exclude SHAPE-incompatible 3D structures, we plotted the INF for our models as a function of correlation between our predicted profile and SHAPE experimental data for each case (Figs. 2.4 and A.4, A.5 in Appendix A). Our findings support the idea that the local interaction pattern may be used to predict SHAPE reactivity. After finding the model in our near-native MD simulations that yielded the highest predicted correlation to SHAPE for each case, we plotted the INF values from the preferred near-native model for all the cases as a function of correlation. Choosing the highest correlated case was a matter of convenience because we attained similar results regardless of which near-native MD case we picked. The 3D structures with lower 
INF trend toward lower correlations than structures with higher INF. This is especially true for the non-native $2 \mathrm{D}$ decoy structures. While the native $2 \mathrm{D}$, non-native $3 \mathrm{D}$ structures sometimes attain higher correlations than our preferred MD model, the invariable benefit of this calculation is that structures with low INF did not have high SHAPE correlation and structures with high INF did not have low correlation. Because native 2D, non-native 3D structures sometimes attained strong correlations even with high RMSD (Figs. A.6 and A.7 in Appendix A) and marginally lower INF, our algorithm cannot be used to select the native or even near-native 3D structure. However, our algorithm can be used to sieve structures and determine whether they are compatible with SHAPE. In addition, we found that our algorithm can use SHAPE data to distinguish between near-native and most 3D structures with non-native $2 \mathrm{D}$ structure.

\subsection{Discussion}

The essence of this method is to capture some key parameters that best describe the mechanism of SHAPE reaction. The correlation between experimental SHAPE data and the predicted SHAPE profile from RNA crystal structure indicates that the nucleotide dynamical ability is the major factor in the SHAPE mechanism. In spite of many successful applications of SHAPE-directed RNA secondary structure prediction [33-37], the application of SHAPE technique to 3D structure modeling is far more complicated. This is partly because the SHAPE mechanism is not fully understood. We found that for the same secondary structure, different 3D structures could have similar predicted SHAPE profiles. As a result, the current 3DSSR function may not be used to predict the native, crystalline structure from 

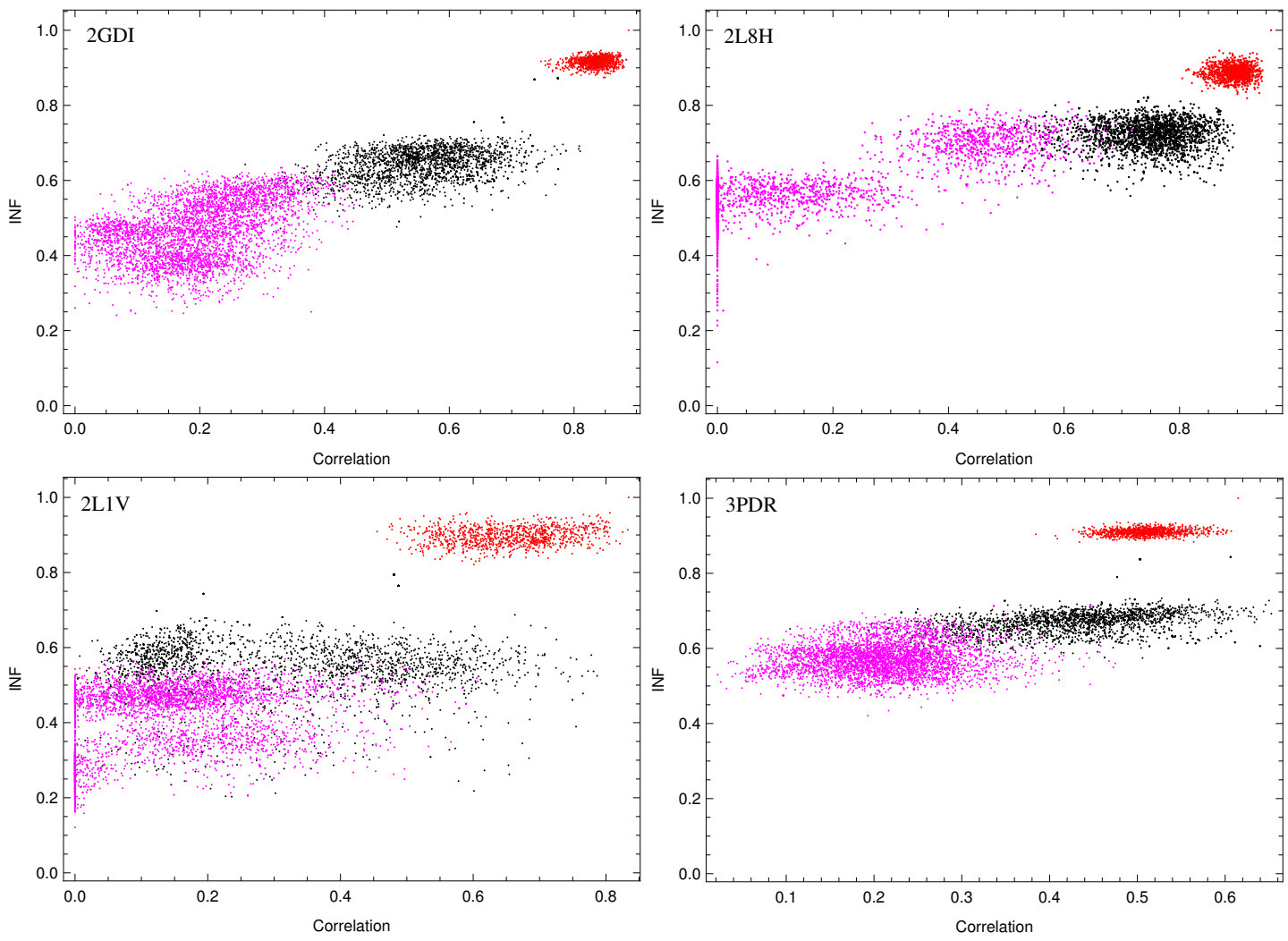

Figure 2.4: Comparison of INF and correlation for the best (top) and worst (bottom) cases that were shown in Fig. 2.3. All the near-native MD cases (red), native 2D, non-native 3D cases (black), and 3D structures with non-native 2D structure (magenta) are displayed for each case. These results show that exclusion of most non-native 2D structures and many non-native 3D structures is possible using the 3DSSR model. 
the near-native conformational ensemble. This is expected because SHAPE is a chemical probe utilized in a solution environment, therefore, many near-native structures can be considered native. However, our algorithm can be used to exclude many $3 \mathrm{D}$ structures that are not compatible with SHAPE, which could be useful, especially when combined with other methods to sieve the pool of low energy structures. More accurate evaluations for the interaction energies are required in order to improve the reliability of distinguishing structured loops from helices (both have low SHAPE reactivities). Furthermore, investigating the change of the SHAPE profile as a function of solution conditions will require more detailed modeling of effects such as temperature and ion concentrations. Nevertheless, large structural changes, caused by ligand-binding, site-mutations, temperature-jumps, etc., can be captured by the change of SHAPE profiles [93]. In such cases, SHAPE probing can provide significant insights into the structure and dynamics.

\section{Interaction search}

Local flexibility and some specific conformations facilitate SHAPE reaction. Information about a single static structure may not be sufficient to give strong correlation with the SHAPE reactivity. The SHAPE reaction mechanism is clearly quite complicated and requires detailed analysis for the dynamics, stability and structure of RNA with a large conformational ensemble. Our 3DSSR-based calculations indicate that flexibility contributes the most to the SHAPE mechanism. Interactions among the nucleotides stabilize the local structure and restrict local sampling ability and this results in lower SHAPE reactivity. Capturing all the interaction types is critical in rebuilding the SHAPE profile from a given RNA 3D structure.

Identifying the various interactions and assigning correct energy intensities are two pri- 
mary issues in this calculation. We apply the RNAview plugin [9] to capture base pair interactions and assign the interaction intensity based on the frequency of specific conformations among known RNA structures. Our current interaction intensity function for stacked bases is only dependent on angle and distance between the bases and is sequenceindependent. Further improvements of the energy function will require a more detailed investigation based on the known RNA structures to extract parameters that are dependent on local sequence and structural motif.

In our calculation, several nucleotides yield false predictions. The interaction intensities at these positions are altered by base-backbone hydrogen bonding or other types of interactions that are not included in the algorithm. Further development of this algorithm should include more types of interactions.

\section{Crystal structure vs. SHAPE reagent-involved solution structure}

Our calculations are based on RNA crystal structures. The premise that solution structures in SHAPE experiments are identical or very close to the crystal structures obtained in the PDB database is inherent in this method. This presumption is not always correct. RNA molecules are quite dynamic in solution because of thermal fluctuations, the presence of possible alternative folds, and the interaction with other solution molecules, such as water and ions. Another issue concerns the ligand: our calculation shows the size of the SHAPE reagent molecule is significantly larger than water molecules. SHAPE-ligand binding may perturb the local RNA structure, but this remains for further investigation.

Our calculation shows that including the ligand accessibility term generally improves the correlation, especially for compact structures such as pseudoknot structures like the M-box riboswitch from $B$. subtilis (2L1V). However, analysis for several nucleotide posi- 

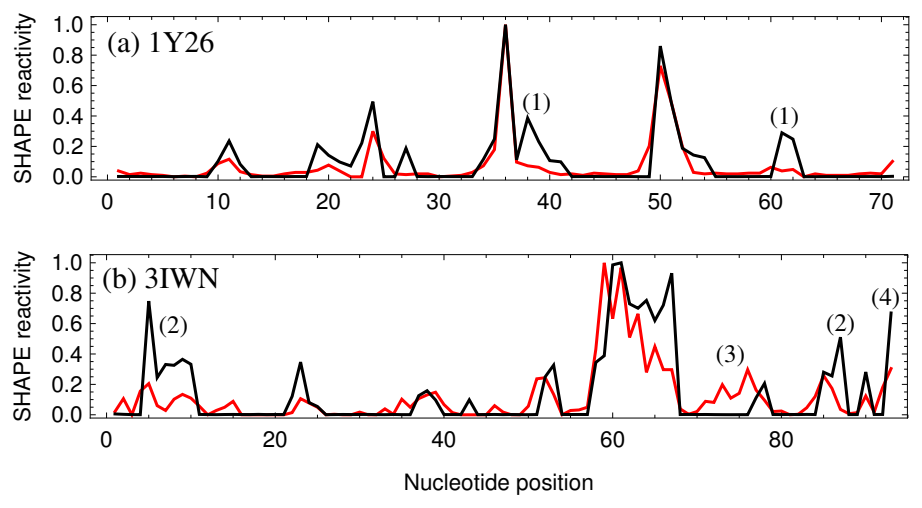

Figure 2.5: (a) Comparison between the 3DSSR-predicted (black) and experimental (red) SHAPE profile for the adenine riboswitch (1Y26) [37], which has a Pearson correlation of 0.88. (b) Comparison between the predicted (black) and experimental (red) SHAPE profile for the cyclic-di-GMP riboswitch from $V$. cholera (3IWN) [36], which has a Pearson correlation of 0.74. Nucleotides marked (1) and (2) have high predicted SHAPE reactivity due to the base-ligand interactions that are not considered in the 3DSSR model. Nucleotides marked (3) are in helix region but have medium SHAPE reactivity. The false prediction for the nucleotide marked (4) is likely due to the effect of the tail not considered in the current 3DSSR function.

tions showed that this calculation may also introduce some error. For some nucleotides, the crystal structure buries the 2'-OH and makes it inaccessible for the ligand binding. However, experimental SHAPE data showed some reactivities for these nucleotides. The RNA molecule probably displays large-scale structural motion in solution. This structure alteration results in higher ligand accessibility and makes nucleotide-ligand binding more probable. The constant $S_{0}$ in the 3DSSR function accounts for the dynamic effect of RNA molecules in solution. Although our results indicate ligand accessibility is not a generally negligible effect, we did find that ligand accessibility is the smallest contributor to the SHAPE mechanism, which is consistent with previous work [32].

\section{SHAPE reactive and inert conformations}

The SHAPE reaction may be exquisitely sensitive to the nucleotide conformation[32]; 
thus, the SHAPE reactivity for a nucleotide is correlated to the probability of the nucleotide to adopt SHAPE-reactive conformations. The flexibility of each nucleotide corresponds to the potential for the nucleotide to access the reactive conformations. The flexibility per se does not provide the actual frequency (probability) for the nucleotide to reach the specific reactive conformations. In an attempt to search for SHAPE-reactive conformations [32], based on the SHAPE profile of the 16S rRNA in crystallized E. coli ribosome ( 1500 nucleotides), McGinnis et al. suggested the existence of several key factors for highly reactive conformations. The study mainly focused on 35 highly reactive nucleotides, and these highly reactive nucleotides are postulated to reveal a portion of the whole SHAPE chemistry.

As shown in Fig. 2.2c, nucleotides U24, U36, and U50 have comparable flexibilities $(1.05,1.77$, and $0.44 \AA)$ but differ significantly in SHAPE reactivity. For example, nucleotide U24 has a much lower SHAPE value than U36 (1.55 to 5.17). We also found that the flexible nucleotide A61 is nearly inert with SHAPE value of 0.2. We propose that conformations adopted by nucleotides U36 and U50 may be close to the respective SHAPE-reactive conformations and are, thus, SHAPE-reactive. In contrast, conformations adopted by nucleotides U24 and A61 may be away from the SHAPE-reactive conformations and are less SHAPE-reactive even though they are flexible. Systematic comparisons between SHAPE profile and nucleotide flexibility for a broad range of diverse RNAs may facilitate the search for SHAPE-reactive and inert conformations.

\section{Investigating electrostatic potential energy}

While the focus of this project is to develop a general sieving method to distinguish SHAPE-compatible structures from SHAPE-incompatible structures, we also attempted to 
determine the reason for the phenomenon of highly reactive nucleotides. From our search, several factors may potentially contribute to anomalously high SHAPE signals for select nucleotides. As previously explained, an absence of constraining forces and high ligand accessibility in tandem with a favorable propensity to sample SHAPE-reactive conformations may lead to a high SHAPE reactivity for some nucleotides. Additionally, the 1M7 molecule is polarized with a partially anionic oxygen covalently bound to the reactive ring carbon. As shown in Fig. 2.6, local neutralization of the anionic backbone charge by cationic factors, such as magnesium ions, might increase the frequency of 1M7 reactions with 2'-hydroxyl groups in regions with higher (less negative) electrostatic potential.

In an idealized scenario, a magnesium ion could kinetically catalyze the SHAPE reaction by neutralizing the negatively charged phosphodiester backbone. This neutralization would allow polarized 1M7 molecules in reactive orientations to more frequently sample the reactive space around the 2'-hydroxyl group because repulsion by the phosphodiester group of the partially negative polar side of the $1 \mathrm{M} 7$ molecule would be lessened. Additionally, a water molecule could act as a bridge between a magnesium ion and the partially anionic oxygen bound to the reactive carbon of $1 \mathrm{M} 7$. If the magnesium ion were appropriately positioned, this coordinating water molecule could anchor the $1 \mathrm{M} 7$ ligand in a favorable orientation by exposing the reactive carbon to the 2'-hydroxyl group and encourage more frequent sampling of the reactive space around the 2'-hydroxyl group. Interaction between a bridging water molecule and the partially anionic oxygen of 1M7 would reduce the activation energy of the reactive carbon and provide chemical catalysis for the reaction (see Fig. 2.6). This conjectured effect may contribute to the hyper-reactivity for nucleotides in the E. coli ribosome (PDB ID: 3i1m)[32], where nucleotides 530-532 are all found to be highly SHAPE-reactive and near a magnesium ion. 

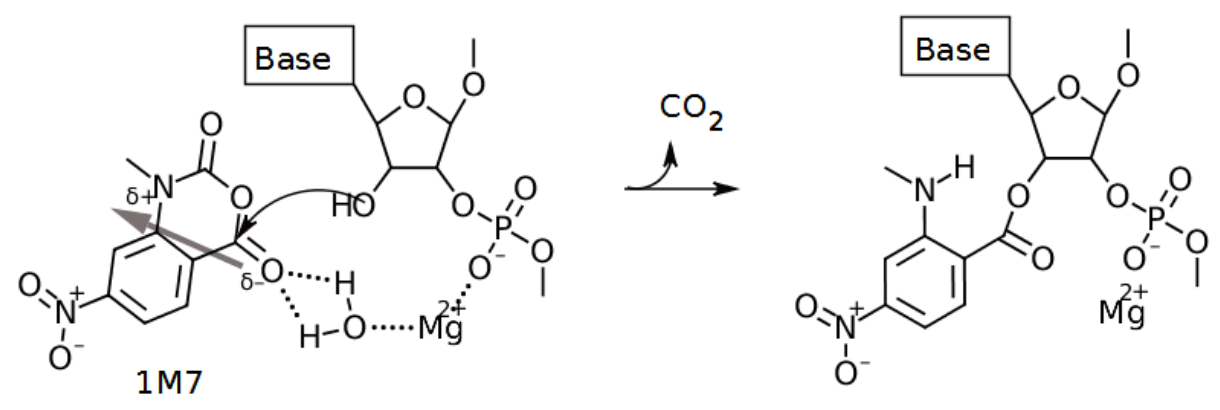

Figure 2.6: In this reaction mechanism, a magnesium ion quenches the negatively charged backbone phosphate group and simultaneously coordinates the reaction of the $1 \mathrm{M} 7$ ligand with the nucleotide through a bridging water molecule. A thick grey arrow shows the dipole moment going through the $1 \mathrm{M} 7$ molecule, which points from the partially anionic oxygen bound to the reacting carbon toward the partially cationic ring nitrogen. If bound to an ideally positioned magnesium ion, a coordinating water molecule may weakly hydrogen bond to the partially anionic oxygen on the 1M7 molecule and facilitate increased sampling of SHAPE-reactive space. The magnesium ion may anchor 1M7 in a reactive orientation so that the reactive carbon collides with the 2'-hydroxyl oxygen until the reaction occurs. If the negatively charged phosphodiester backbone is not quenched, the polarized 1M7 molecule would be more likely to sample the reactive space in an unfavorable (nonreactive) orientation. An arced arrow shows the movement of electrons from the 2'-hydroxyl oxygen to form a bond with the reactive carbon. In this picture, the magnesium ion behaves as a kinetic catalyst by providing a means for SHAPE ligands to more frequently sample the reactive space in a favorable orientation, and it acts as a weak chemical catalyst by pulling electrons from the partially negative oxygen through the hydrogen bond with water and increasing the reactivity of the carbon. 
Using a tiered calculation to compare the electrostatic potential energy to the SHAPE profile, we found that the 2'-hydroxyl group of many highly reactive SHAPE nucleotides have higher average electrostatic potential energy. In contrast to our other metrics that take into account the dynamic propensity of each nucleotide, this measure corresponds to the ability of a nucleotide to attract the reactive part of the SHAPE reagent to the 2'-hydroxyl reaction site.

However, the local electrostatic potential energy is not immediately viable to be included in the 3DSSR function because it is highly sensitive to small conformational changes, so calculating the electrostatic potential energy profile for near-native MD structures can give wildly different results from one structure to the next. In our calculation, we utilized MD structures to find the average electrostatic potential energy profile for each RNA molecule. Although we averaged the electrostatic potential energy, several regions of high electrostatic potential energy correspond to helix regions, which are generally SHAPE inert. Furthermore, the electrostatic potential energy is generally higher on the surface of the RNA molecule, where the concentration of negatively charged, phosphate backbone atoms is lower, which means that the electrostatic potential energy may be correlated with both solvent accessibility and constraining interactions considered in the 3DSSR.

It is important to note that our calculations could not discern whether the electrostatic potential energy is an effect contributing to the physical SHAPE mechanism or if this trend is an artifact of the correlation between the electrostatic potential energy, the solvent accessibility, and the number of constraining interactions for a given nucleotide. Therefore, this measure is not robust enough to account for the full SHAPE mechanism, and we consider nucleotide dynamics to be the primary driving force behind SHAPE reactivity. However, our search leads us to suggest that if the 2'-hydroxyl site on a flexible nucleotide is in 
a region of high electrostatic potential energy, the nucleotide may react more strongly to SHAPE than flexible nucleotides in regions with low electrostatic potential energy.

\section{Future improvements to the 3DSSR function}

Our interaction intensity function constitutes a lowest order approach at scoring the energy. Because we do not calculate the global free energy of the RNA to build a predicted SHAPE profile, we can use this approximation for individual RNA nucleotides to roughly measure the relative strength of their constraints. In the helix regions, the SHAPE reactivity is consistently low and accounted for by known secondary structure information (the $B P(n)$ function). As shown in Table 2.1, our algorithm might be able to consider, to the lowest order approximation, tertiary interaction effects and predicts relative loop region reactivity. For the $B P(n)$ function alone, the average correlation is only 0.49 , while the entire 3DSSR prediction yields an average correlation of 0.78 ; without including the $B P(n)$ function, we reach a slightly lower average correlation of 0.75 . The improvement of the 3DSSR function, which accounts for tertiary effects, over the $B P(n)$ function is mainly from the predictions for loop nucleotides. The 3DSSR algorithm would benefit from a more rigorous energy function in the future, but our results indicate that we can currently distinguish SHAPE-compatible structures from SHAPE-incompatible structures to a useful degree.

The predicted profile using 3DSSR strongly correlates to the experimental SHAPE data, as shown in Fig. 2.3, indicating that the three factors (interaction intensity, base pairing pattern and ligand accessible surface) in 3DSSR are the major contributors to SHAPE reactivity. The current algorithm for interaction intensity calculations contains the major interactions involved in RNA: base pairing and base stacking. However, several false pre- 
dictions of major peaks are shown in Figs. 2.5 and 2.7. The results could be improved if the following interactions are considered.

Base-ligand interactions: While many molecules strongly interact with RNA, ligand binding is not considered in this model. For the adenine riboswitch (1Y26) shown in Fig. 2.5a, the 3DSSR function falsely predicts high SHAPE reactivities for nucleotides 38-40, 61, and 62. In Fig. 2.1a, we see that these nucleotides are interacting with a ligand molecule in the 3-way junction of 1Y26. Similarly, the Cyclic-di-GMP riboswitch from V. cholera (3IWN) has ligand interactions at nucleotides 5-9 and 87 in Fig. 2.5b. In all of these cases, the 3DSSR function predicts higher relative SHAPE reactivities than is experimentally observed. Lack of detailed base-ligand interactions in 3DSSR may be a factor contributing to the over-estimation. In the future, we must take into account the constraining effects of other molecules on the nucleotide flexibility. If the base-ligand and base-ion interactions can be accurately estimated, the 3DSSR-based prediction is expected to better correlate with experimental SHAPE data.

Non-WWc stacking: As shown in Fig. 2.7b of the lysine riboswitch T.maritima (3DIG), nucleotides 21-29 and 65-72 have much higher predicted relative SHAPE reactivities than is experimentally observed. These nucleotides are constrained by the non-WWc base stacking shown in Fig. 2.7a. Like A-form helices, this type of base stacking may also effectively constrain nucleotides. However, our current algorithm underestimates the interaction intensity of the non-WWc stacking and overestimates relative SHAPE reactivities when compared to experimental SHAPE data.

Base-backbone interaction: Currently, the interaction intensity calculation contains base-base interactions only. Base-backbone and backbone-backbone interactions are not considered. As shown in Fig. 2.7a, the base group of nucleotide G62 forms a hydrogen 
(a)

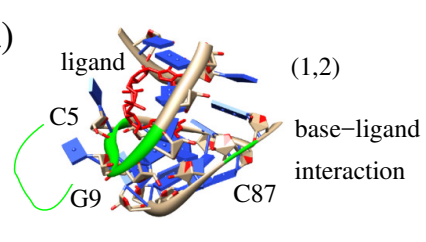

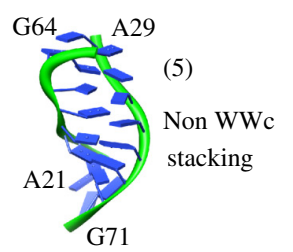
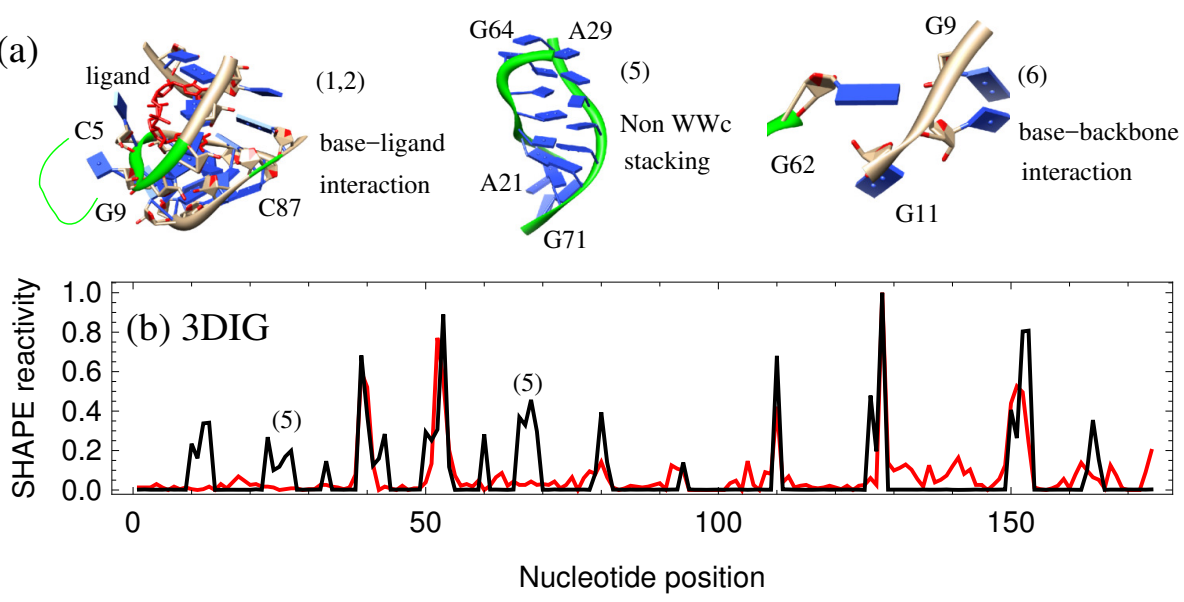

Figure 2.7: (a) 3D structures for some of the interactions not considered in the 3DSSR function: base-ligand interactions for (1) and (2) in Fig. 2.5; non-WWc stacking for (5) in Fig. 2.7b and base-backbone interactions between the nucleotide G62 and nucleotides G9-G11 (6) in the TPP riboswitch from E. coli (2GDI). (b) Comparison of the predicted (black) and experimental (red) SHAPE profile for the lysine riboswitch from T. maritime (3DIG) [36], which has a Pearson correlation of 0.70 . The false prediction for the nucleotides marked (5) is likely caused by the under-estimation of the interaction intensity within the non-WWc stacking region shown in (a).

bond with the backbone of nucleotide $10 \mathrm{U}$ in the TPP riboswitch from E. coli (2GDI). Because this stabilizing interaction is not included in the current 3DSSR model, we predicted higher relative SHAPE reactivities for G62 and 10U than are experimentally observed (see Fig. 2.3).

Tail effects: During SHAPE probing, additional sequences at 5' and 3' ends are added to the RNA of study. These elongating sequences could possibly form interactions with terminal RNA nucleotides and attenuate their experimental SHAPE reactivity. Non-helical nucleotides are especially susceptible to this effect, and the current 3DSSR function does not account for additional tail sequences. In Fig. $2.5 \mathrm{~b}$, the last nucleotide of the Cyclicdi-GMP riboswitch from $V$. cholera (3IWN) is predicted to have a higher relative SHAPE reactivity than is indicated by the experimental data. Future development of the 3DSSR function should account for the tail effects. 


\subsection{Conclusion}

Chemical structure mapping methods, such as SHAPE, provide useful insight into the structure and dynamics of RNA molecules. Using MD simulations, we first verified that SHAPE reactivity is correlated with nucleotide position and local nucleotide mobility by being unreactive in rigid helices and reactive in flexible loops (Figs. 2.1 and 2.2). Next, we used structural information - the aforementioned interaction intensity, base pairing pattern, and solvent accessibility — to build the 3D Structure-SHAPE Relationship model (3DSSR) that can predict SHAPE profiles from individual RNA structures (Figs. 2.3, 2.5, and 2.7). Then, we tested the 3DSSR on near-native 3D structures, non-native 3D structures with correct $2 \mathrm{D}$ structures, and non-native $3 \mathrm{D}$ structures with incorrect $2 \mathrm{D}$ structures to show that we can use the 3DSSR to separate structures that are incompatible with SHAPE data from SHAPE-compatible structures (Fig. 2.4). While our approach cannot determine the native crystal structure from SHAPE data, we can use SHAPE data to exclude incompatible structures from consideration, which may contribute towards using chemical mapping methods for RNA 3D structure analysis and prediction. 


\section{Chapter 3}

\section{Analytical modeling and deep learning approaches to estimating RNA SHAPE reactivity from 3D structure}

This chapter was published ${ }^{1}$.

Summary: The selective 2'-hydroxyl acylation analyzed by primer extension (SHAPE) chemical probing method provides information about RNA structure and dynamics at single nucleotide resolution. To facilitate understanding of the relationship between nucleotide flexibility, SHAPE reactivity, and RNA 3D structure, we developed an analytical 3D Structure-SHAPE Relationship (3DSSR) method and a predictive convolutional neural network (CNN) model that predict the SHAPE reactivity from RNA $3 D$ structures. Starting from an RNA 3D structure, the analytical model combines key factors into a composite

\footnotetext{
${ }^{1}$ Travis Hurst, Yuanzhe Zhou, and Shi-Jie Chen. "Analytical modeling and deep learning approaches to estimating RNA SHAPE reactivity from 3D structure". In: Commun. Inf. Syst. 19.3 (2019), pp. 299-319. DOI: https://dx.doi .org/10.4310/CIS.2019.v19.n3.a4. URL: https://www . intlpress.com/site/pub/pages/journals/items/cis/content/vols/0019/0003/ a.004/index.php.
} 
function to predict conformational flexibility of each nucleotide and calculate the correlation between the prediction and experimental SHAPE reactivity. Here, we apply the 3DSSR and the deep learning SHAPE model to SHAPE data-assisted RNA 3D structure prediction. We show that the models provide an effective sieve to exclude $3 D$ structures that are incompatible with experimental SHAPE data. Additionally, we compare the 3DSSR analytical model with the CNN deep learning model that recognizes structural and physical/chemical patterns to predict SHAPE data from RNA 3D structure. Depending on the training data set, the analytical model outperforms the deep learning approach for most test cases, indicating that insufficient data is available to adequately train the CNN at this juncture. For other test cases, the deep learning approach provides better predictions than the analytical model, suggesting that the deep learning approach may become increasingly promising as more SHAPE data becomes available.

\subsection{Introduction}

Galvanized by recent progress in RNA chemical probing technology, researchers developed efficient, data-driven experimental modeling approaches that place effective constraints on RNA structure to complement established template and physics-based methods [22-26]. Selective 2'-hydroxyl acylation analyzed by primer extension (SHAPE) provides significant insights into local nucleotide structure and dynamics in RNA [27, 28]. SHAPE reagents are small ligands—such as 1-methyl-7-nitroisatoic anhydride (1M7) [94]—that covalently bind to the 2'-hydroxyl group of a nucleotide (see Fig. 3.1) [29]. Previous studies [30-32] suggest that unconstrained nucleotides have a greater ability to sample more conformations and to adopt SHAPE-reactive postures, which causes them to have higher 
SHAPE reactivity. In contrast, nucleotides that are constrained by base-pairing and stacking interactions have a lower propensity to sample a variety of poses and are much less reactive. By quantitatively measuring local nucleotide dynamics, SHAPE is an effective tool for probing whether a nucleotide is constrained by interactions with other nucleotides (in a helix or structured loop) or is located in a flexible loop/junction, without many interactions. In secondary structure modeling, use of SHAPE data substantially improves accuracy and efficiency [33-37], where SHAPE reactivity is used to provide additional structural constraints for free-energy based predictions [38]. Moreover, when used as the basis for advanced experimental approaches, such as differential SHAPE reactivity, mutate-andmap, and time-resolved SHAPE chemistry, SHAPE probing provides helpful information for the in vitro and in vivo determination of non-canonical tertiary interactions and RNA kinetics[39-45].

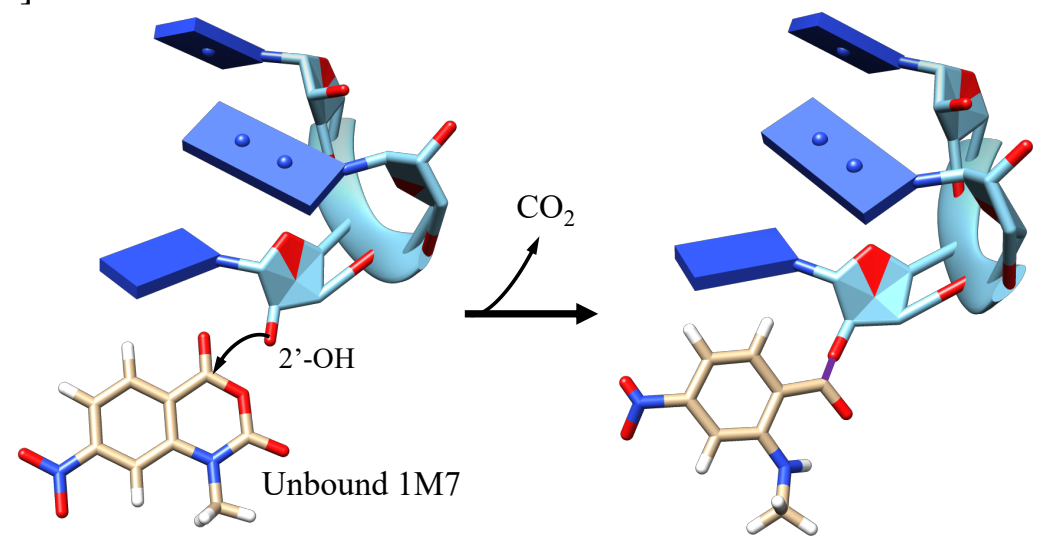

Figure 3.1: The SHAPE reaction. The RNA nucleotide 2'-OH group attacks the reactive carbon of $1 \mathrm{M} 7$, releases $\mathrm{CO}_{2}$, and forms a covalent bond (purple) with the SHAPE reagent.

Machine learning is a general method of data analysis that automates analytical model building and is based on the idea that models can learn from data, extract patterns, and make decisions with minimal human intervention. Complex problems without clear underlying mathematical structures benefit from machine learning because manually constructed analytical models cannot easily capture all of the underlying mechanics. The appeal of ma- 
chine learning methods is the ability to derive predictive models without a need for strong assumptions about underlying mechanisms, which are frequently unknown or insufficiently defined in computational biology. Machine learning has exhibited unprecedented performance in protein structure prediction [95-100], protein-ligand binding [101-104], regulatory genomics and cellular imaging $[105,106]$. Deep learning is a subset of machine learning based on artificial neural networks, and "deep" refers to the presence of multiple hidden layers. The convolutional neural network (CNN) is one of the deep learning network models and has gained significant attention due to its success in computer visual recognition.

Previously, we developed an analytical function to quantitatively predict the SHAPE profile from individual RNA 3D structures [78]. We showed how our function can be applied to exclude SHAPE-incompatible structures. To establish the relationship between SHAPE reactivity and nucleotide dynamics, we generated conformational ensembles with MD simulations to measure the correlation between SHAPE reactivity and the conformational propensity of each nucleotide. Then, by combining key factors that account for physical properties implicated in the SHAPE mechanism-the nucleotide interaction strength, SHAPE ligand accessibility, and base-pairing pattern-we developed the analytical 3D Structure-SHAPE Relationship (3DSSR) function, which characterizes the local nucleotide flexibility and predicts SHAPE reactivity based on information about the nucleotide posture and local energetics. To test the discriminating ability of our tool, we used the 3DSSR function to show how SHAPE-incompatible decoy structures may be excluded based on the low correlation between their predicted SHAPE profile and experimental SHAPE data.

Here, we revisit the 3DSSR model and develop a novel convolutional neural network (CNN) model, which uses experimental structural data to predict the SHAPE reactivity for 
any given nucleotide. First, we briefly describe the formulation of the 3DSSR model on a molecule that was not originally used to test or train either the 3DSSR or CNN model. Then, we describe the methods used to obtain the CNN model. Finally, we compare the ability of the two models to make useful predictions of SHAPE reactivity on RNA molecules used in training and a molecule neither algorithm has seen, emphasizing that analytical formulations often provide more insight than pattern recognition methods when limited data is available.

Table 3.1: RNA structures used for validation. The Protein Database ID (PDBID), length of the RNA in nucleotides (nt), type of RNA, and organism of origin are displayed. The SHAPE profiles for these RNA molecules are from the published experimental data [30, 36, 37, 107, 108].

\begin{tabular}{|l|c|c|c|}
\hline PDBID & Length (nt) & Type of RNA & Organism \\
\hline 2L8H & 29 & TAR RNA & HIV-1 \\
1AUD & 30 & U1A protein binding site RNA & H. sapiens \\
2L1V & 36 & M-box riboswitch & B. subtilis \\
2K95* & 48 & Telomerase pseudoknot & H. sapiens \\
1Y26 & 71 & Adenine riboswitch & V. vulnificus \\
1VTQ & 75 & PreQ1 riboswitch aptamer & B. subtilis \\
1EHZ & 76 & Aspartate tRNA & Yeast \\
1P5O* & 77 & IRES Domain II & Hepatitis $C$ \\
2GDI & 79 & TPP riboswitch & E. coli \\
3IWN & 93 & Cyclic-di-GMP riboswitch & V. cholera \\
4KQY & 117 & SAM-I riboswitch & B. subtilis \\
1C2X* & 120 & 5S rRNA & E. coli \\
3IVK* & 128 & Catalytic core of RNA polymerase ribozyme & E. coli \\
1NBS & 154 & Specificity domain of Ribonuclease P RNA & B. subtilis \\
3PDR & 154 & M-box riboswitch & B. subtilis \\
1GID* & 158 & Group 1 Ribozyme & Synthetic \\
3P49* & 169 & Glycine Riboswitch & H. sapiens \\
3DIG & 174 & Lysine riboswitch & T. maritima \\
4UE5* & 299 & SRP RNA & C. lupus \\
3G78* & 421 & Group II intron & O. iheyensis \\
\hline
\end{tabular}

* Denotes cases used to parameterize the CNN model, not the 3DSSR model. 


\subsection{Methods}

\subsubsection{Finding structures corresponding to SHAPE data}

In order to find RNA structures that correspond to our SHAPE sequences, we used the sequence searching interface equipped with NCBI's BLAST (Basic Local Alignment Search Tool) program [109] provided by RCSB protein databank [110] to align the sequences. In the 3DSSR (CNN20) model 12 (20) RNA structures with an average length of $~ 92(120)$ nucleotides that have SHAPE reactivity data were used (see Table 3.1). For comparison, we also parameterized the CNN model using the same 12 structures as 3DSSR (CNN12). SHAPE reactivity data came from databases for sharing nucleic acid chemical probing data, the RNA mapping database (RMDB) [108] and the SNRNASM database [107]. To have comparable SHAPE reactivity values between different RNA structures, all of the negative values of SHAPE reactivity data are set to zero, in accordance with previous work [34]. Furthermore, the SHAPE profiles are scaled by the maximum reactivity value of each respective RNA structure, which confines SHAPE reactivity data to range from 0 to 1.

\subsubsection{Reviewing 3DSSR methods and conclusions}

Previously, we used simulations to show that SHAPE data corresponds with nucleotide flexibility, parameterize the 3DSSR model, and generate decoys to illustrate how our model can be used to exclude SHAPE-incompatible structures [78]. The ability of a nucleotide to react with SHAPE depends on the propensity of a nucleotide to sample SHAPE-reactive postures and the ability of the SHAPE ligand to access the reactive site. Capturing these 
A)

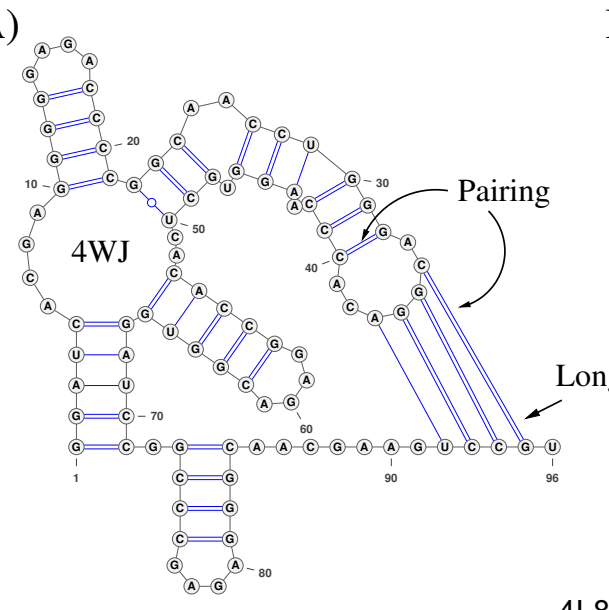

B)

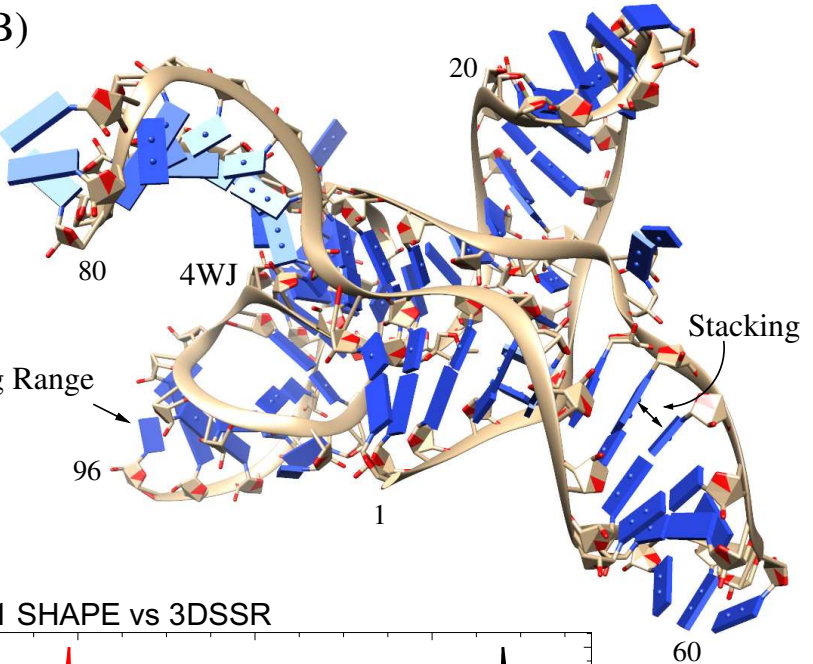

C) 1.0

4L81 SHAPE vs 3DSSR

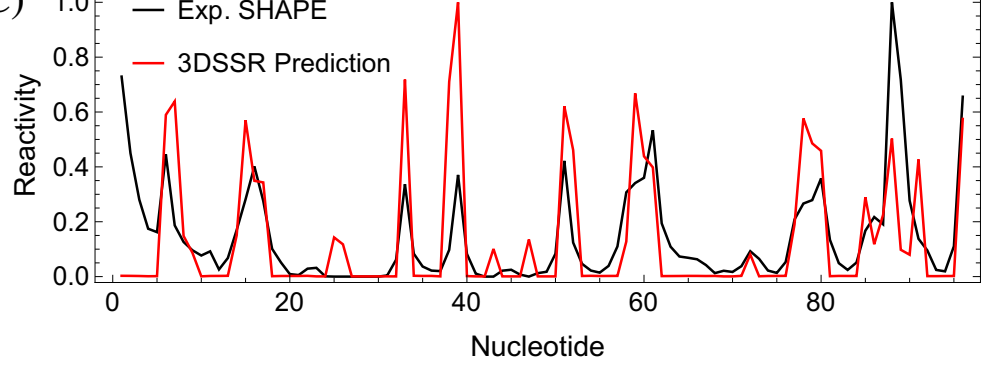

Figure 3.2: The 2D, 3D, and SHAPE reactivity of RNA-Puzzle 8 (PDBID: 4L81). A) The 2D structure [111] shows the four-way junction (4WJ), base-pairs, and long range interactions. B) The $3 \mathrm{D}$ structure shows the $4 \mathrm{WJ}$ and an example of a base stacking interaction. C) The experimental and predicted SHAPE profiles for the crystallized 4L81 structure show good agreement (Pearson correlation $=0.57$ ). 
concepts, we proposed the 3DSSR function

$$
P(n)=B P(n) \cdot \frac{S A S(n)+S_{0}}{|I I(n)-1.0|}
$$

to estimate the nucleotide stability and predict the SHAPE reactivity $P(n)$ for a nucleotide $n$. The base-pairing factor $B P(n)$ accounts for the 2D structure, which is characterized by the base-pairing pattern: a nucleotide $n$ in a helix region is assigned $B P(n)=0.01$ and a nucleotide in a loop or junction region is assigned $B P(n)=1.0$. A $2 \mathrm{D}$ structure can always be extracted from a 3D structure (for example, using the RNApdbee 2.0 webserver [112]), and helix nucleotides are normally SHAPE-inert. The SHAPE ligand accessible 2'-OH surface area $S A S(n)$ describes the necessary requirement of a SHAPE ligand to access the nucleotide for a reaction to occur. If a nucleotide 2' $-\mathrm{OH}$ is buried inside the RNA structure, SHAPE reagents cannot react, which reduces the SHAPE reactivity. The unbound SHAPE ligand has an effective radius between 2.0 and $2.5 \AA$, and our results indicate that the 3DSSR function is not sensitive to different probe sizes within this range. The accessible surface of 2'-OH is calculated using VMD [85]. $S_{0}$ is a constant, accounting for the ability of a nucleotide to become accessible during experimental SHAPE probing. $I I(n)$ is the interaction intensity for nucleotide $n$, which accounts for tertiary structure interactions. Through fitting, information from base-pairing and base-stacking interactions are combined to calculate the $I I(n)$, a quasi-energy score for each nucleotide.

In the present study, we focus on a SHAPE data-assisted approach to RNA 3D structure prediction. For a given RNA sequence, we can generate an ensemble of possible conformations using, for example, the IsRNA coarse grained simulation model [53]. We then score each conformation by the correlation (similarity) between the (3DSSR-predicted) SHAPE profile of the conformation and the experimentally determined SHAPE data for the RNA 
molecule. Although due to the low-resolution energy model, the 3DSSR model might not be able to identify the native, crystal structure from SHAPE data alone, as shown below, the model can assist structure prediction by successfully excluding SHAPE-incompatible structures.

\subsubsection{Applying the model to the SAM-I/IV riboswitch aptamer}

For illustration, here we apply the 3DSSR model to the SAM-I/IV riboswitch aptamer (PDBID: 4L81) that was used in round 8 of RNA-Puzzles [1] (see Fig. 3.2), a communitywide, CASP-like blind test for RNA 3D structure prediction. This structure has not been previously used to train or test the 3DSSR model, and the structures submitted in the RNAPuzzle competition by different labs give us objective decoys to show the ability of the 3DSSR model to exclude structures that are incompatible with SHAPE.

First, we access the submitted structures and assessment results from the RNA-Puzzles database (see Fig. 3.3A for a structure submitted to the competition). Next, we extract the 2D structures from the submitted 3D structures using the RNApdbee 2.0 webserver [112] (Fig. 3.3B). After that, we use RNAview software to identify the base pair types from the 3D structures [9] (Fig. 3.3C). Additionally, we directly calculate the stacking interaction information from the 3D structures: the angles and distances between different RNA bases (Fig. 3.3D). Then, we calculate the solvent accessible surface of each nucleotide 2'-OH in the 3D structures with VMD [85] (see Fig. 3.3E for a visual representation). Finally, we use the 3DSSR function to combine all of the structural information and predict the SHAPE reactivity for each nucleotide (Fig. 3.3F). To evaluate the SHAPE-compatibility, we also calculate the Pearson correlation between the experimental and 3DSSR-predicted SHAPE profiles. Comparison of the 3DSSR-predicted and experimental SHAPE profiles on the 
native, crystal structure can be seen in Fig. 3.2C. Provided with candidate 3D structures and experimental SHAPE data, we can exclude SHAPE-incompatible structures on the basis of their 3DSSR-predicted SHAPE profile.

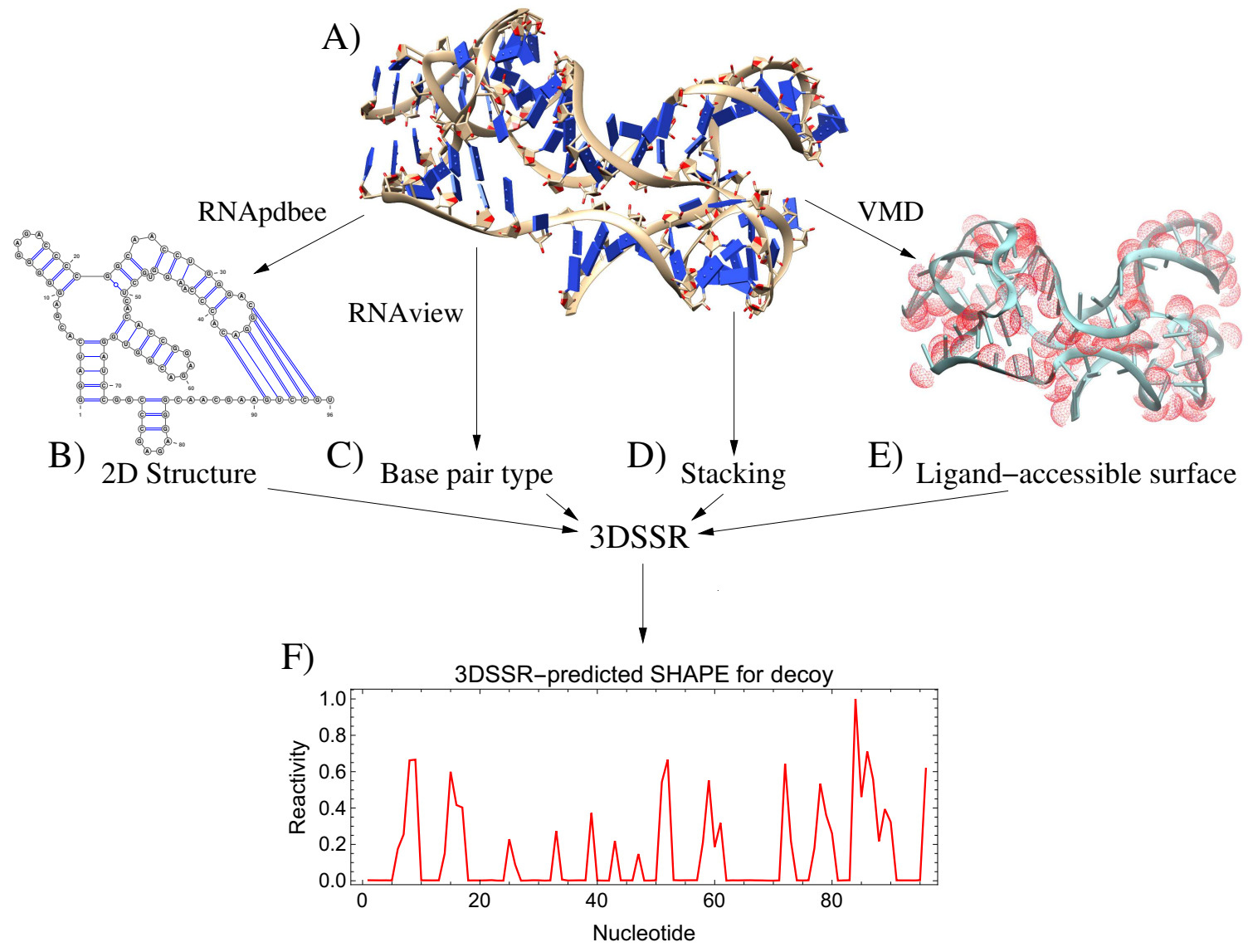

Figure 3.3: 3DSSR workflow on an RNA-Puzzle 8 decoy. A) A candidate 3D structure decoy is processed by RNApdbee 2.0 [112], RNAview [9], in-house software, and VMD [85] to B) produce a 2D structure, C) identify base pair types, D) extract stacking angle/distance information, and E) calculate the solvent accessible surface of the 2'-OH, respectively. The information extracted from the structure is input into the 3DSSR function to produce F) the predicted SHAPE profile for each nucleotide.

The sensitivity of the model to structures with high RMSD and lower Interaction Network Fidelity (INF; a quantity to measure the similarity in interaction pattern) [113] can be seen in Fig. 3.5, where we apply the 3DSSR model on all of the submitted structures for 
RNA-Puzzle 8 to show the ability of the 3DSSR function to exclude SHAPE-incompatible structures. Contributing to the objectivity of the test, the submitted 3D structures and assessment results (values of RMSD and INF for each structure) for RNA-Puzzle 8 were all taken from the RNA-Puzzles database. The results suggest that many of the 43 submitted structures could be discarded because they are incompatible with SHAPE. For example, the native crystal structure is ranked in the top ten, and we could comfortably discard the bottom 20 structures, which all have a correlation $<0.45$. Only one structure ranked in the bottom 20 by the 3DSSR model has RMSD (INF) $<(>) 11.2(0.80)$, and no structure in the bottom 20 has favorable assessment values for both RMSD and INF. As can be seen in Fig. 3.5A, the combination of assessment results indicate that a cutoff of 0.45 is quite conservative. We could discard the bottom 65 percent of structures (the bottom 28), which would keep all of the structures with favorable assessment results for both RMSD and INF.

For RNA-Puzzle 8, discarding more than the bottom 70 percent would cause us to discard the native structure. However, the quality of the SHAPE data, the size of the RNA, and the quality of candidate structures all affect the number of structures that may be comfortably excluded on the basis of SHAPE data using 3DSSR. These factors should be known so that a reasonable sieving scheme can be found.

\subsubsection{Using a CNN to predict SHAPE reactivity from structure}

\section{Describing the nucleotide environment}

In agreement with SHAPE experiments, our CNN method probes RNA structure at single nucleotide resolution. For each nucleotide, the surrounding environment refers to neighboring atoms within a cubic volume of space around the nucleotide. Since we define the 
space surrounding a nucleotide as the space confined in a cube, the environment captured by this cube is not rotationally invariant. To remove the effects caused by the different choices of the cube orientation, we set a local Cartesian coordinate system for every given nucleotide. The coordinate system of a nucleotide is determined by the $\mathrm{C}^{\prime}, \mathrm{C}^{\prime}$, and $\mathrm{O}^{\prime}$ atoms. Specifically, the origin of the local coordinate system is located at the atom $\mathrm{O}^{\prime}$, and the local $\mathbf{x}, \mathbf{y}$, and $\mathbf{z}$ axes are defined as follows. First, we denote the $\mathbf{r}_{\mathbf{C 1 ^ { \prime }}}, \mathbf{r}_{\mathbf{C 4 ^ { \prime }}}$, and $\mathbf{r}_{\mathrm{O}^{\prime}}$ as the coordinates of the selected atoms, $\mathrm{C}^{\prime}, \mathrm{C}^{\prime}$, and $\mathrm{O}^{\prime}$. Second, we calculate three vectors $\mathbf{v}_{\mathbf{x}}, \mathbf{v}_{\mathbf{y}}$ and $\mathbf{v}_{\mathbf{z}}$ with respect to the local origin as

$$
\begin{array}{r}
\mathbf{v}_{\mathbf{x}}=\mathbf{r}_{\mathbf{C 4 ^ { \prime }}}-\mathbf{r}_{\mathrm{O}^{\prime}} \\
\mathbf{v}_{\mathbf{y}}=\mathbf{r}_{\mathbf{C 1 ^ { \prime }}}-\mathbf{r}_{\mathbf{O 4 ^ { \prime }}} \\
\mathbf{v}_{\mathbf{z}}=\mathbf{v}_{\mathbf{x}} \times \mathbf{v}_{\mathbf{y}}
\end{array}
$$

where $\mathbf{v}_{\mathbf{x}}$ represents the vector from atom $\mathrm{O}^{\prime}$ to atom $\mathrm{C}^{\prime}, \mathbf{v}_{\mathbf{y}}$ represents the vector from atom $\mathrm{O}^{\prime}$ to atom $\mathrm{C}^{\prime}$ and $\mathbf{v}_{\mathbf{z}}$ is just the cross product of $\mathbf{v}_{\mathbf{x}}$ and $\mathbf{v}_{\mathbf{y}}$. Then, the $\mathbf{x}, \mathbf{y}$ and $\mathbf{z}$ axes are set according to the following Eq. 3.3,

$$
\begin{aligned}
& \mathbf{x}=\frac{\mathbf{v}_{\mathbf{x}}}{\left\|\mathbf{v}_{\mathbf{x}}\right\|} \\
& \mathbf{z}=\frac{\mathbf{v}_{\mathbf{z}}}{\left\|\mathbf{v}_{\mathbf{z}}\right\|} \\
& \mathbf{y}=\mathbf{z} \times \mathbf{x}
\end{aligned}
$$

The surrounding environment of each nucleotide is captured through a cube centered and oriented according to the local coordinate system. As shown in Fig. 3.4, the length of the cube is $24 \AA$ and the atoms contained in the cube will be used to generate the image for CNN model. 


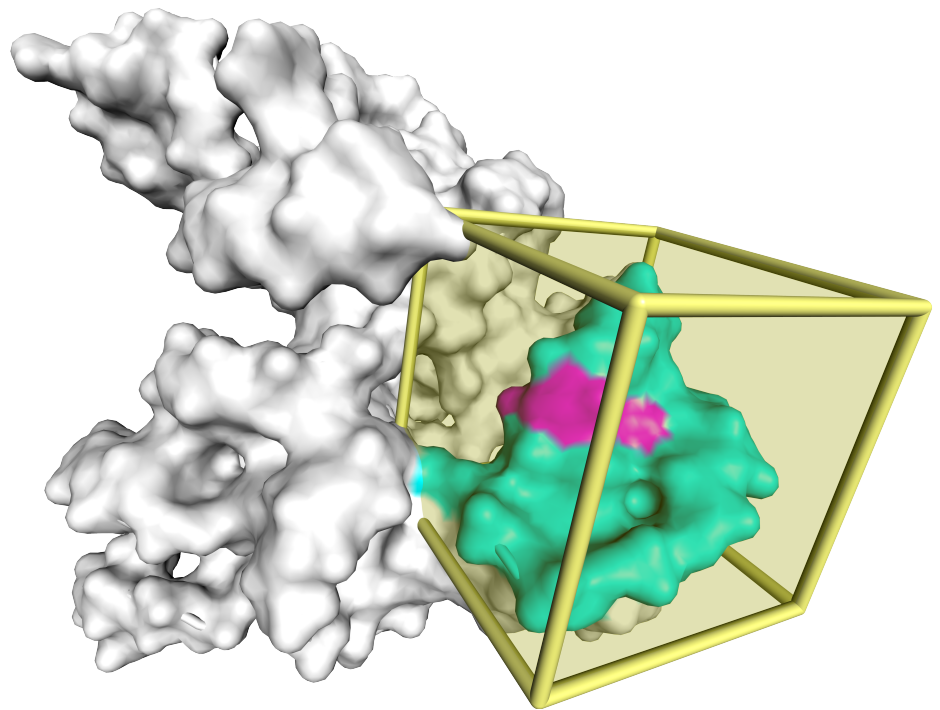

Figure 3.4: . Extracting the 3D image of an RNA nucleotide. The magenta color depicts the nucleotide under assessment and the surrounding environment is confined within the cube with length $24 \AA$. The surrounding atoms are drawn in cyan, and the cube boundaries are drawn with yellow solid lines.

\section{Input: defining the 3D image as input into the $\mathrm{CNN}$}

As we described in the previous section, a $24 \AA \times 24 \AA \times 24 \AA$ cube is used to provide the surrounding environment of each nucleotide. The corresponding image associated with this nucleotide is contained within this cube. As a normal 2D digital image has three color channels (RGB) with each channel represented by a 2D pixel matrix, the 3D image that we used to capture the surrounding environment is also composed of multiple channels. However, our 3D images do not simply use RGB color channels: the channels we selected represent certain physical or chemical features. In our CNN model, we defined 5 channels, which are fully described in Table 3.2.

Since we extract our 3D image from a cube, each channel of the 3D image is represented by a $3 \mathrm{D}$ matrix, and each position in this $3 \mathrm{D}$ matrix has a voxel (3D pixel) value. We set the length of our $3 \mathrm{D}$ image equal to the cube with an image resolution of $1 \AA$, so each 
Table 3.2: Feature channels used for 3D images.

\begin{tabular}{ll}
\hline Feature & Description \\
\hline Hydrophobic & Aliphatic or aromatic carbon atoms \\
Aromatic & Aromatic carbon atoms \\
Positive ionizable & Gasteiger positive charge \\
Negative ionizable & Gasteiger negative charge \\
Excluded volume & All atom types \\
\hline
\end{tabular}

voxel has a dimension of $1 \AA \times 1 \AA \times 1 \AA$. A step function fills the voxels of each channel. For example, the voxels of the excluded volume channel that are occupied by RNA atoms are filled with 1 , and the rest are filled with 0 , according to their Van der Waals radius. A similar procedure was used to generate other channels.

\section{Describing the $\mathrm{CNN}$ architecture}

Our CNN model takes the multi-channel images as input, and outputs a predicted SHAPE reactivity for each image. The network is a basic ResNet [114] architecture with only slight modifications and has 10 convolutional layers. The detailed architecture is shown in Table 3.3. The first layer accepts the 3D image in a convolutional layer and has $647 \times 7 \times$ 7 filters with a stride of 2 . The next layer has 4 residual blocks, with each block containing two convolutional layers. Downsampling is directly performed in the first convolutional layer and by the beginning convolutional layers of blocks 2-4. Finally, the network ends with a global average pooling layer and a 512-way fully-connected layer with a sigmoid activation function. Except the first layer, all of the convolutional layers use $3 \times 3 \times 3$ sized filters. Batch normalization [115] was applied right after each convolutional layer and before 'Rectified Linear Unit' [116] activation, following [115]. In our network, two hidden layers inserted residual shortcut connections for every block. The shortcut takes an identical input from the previous block and maps this identity shortcut right before the 
activation of the second hidden layer within the block; the block is same as the original ResNet block [114]. We initialize the weights as in [114, 117] and train all residual nets from scratch. The only preprocessing we used is the subtraction of a mean value from each image. This mean value is calculated by averaging all the voxels of all images in the training set.

Table 3.3: Details of CNN Architectures. Each building block is shown with two convolutional layers. Downsampling is performed in every convolutional layer with a stride of 2 .

\begin{tabular}{l|l|l|l|l}
\hline & Layer name & Output size & Filter size & Filter num \\
\hline \hline first layer & conv1 & $12 \times 12 \times 12$ & $7 \times 7 \times 7$ & 64, stride 2 \\
\hline block1 & conv2 & $12 \times 12 \times 12$ & $3 \times 3 \times 3$ & 64, stride 1 \\
\hline block1 & conv3 & $12 \times 12 \times 12$ & $3 \times 3 \times 3$ & 64, stride 1 \\
\hline block2 & conv4 & $6 \times 6 \times 6$ & $3 \times 3 \times 3$ & 128, stride 2 \\
\hline block2 & conv5 & $6 \times 6 \times 6$ & $3 \times 3 \times 3$ & 128, stride 1 \\
\hline block3 & conv6 & $3 \times 3 \times 3$ & $3 \times 3 \times 3$ & 256, stride 2 \\
\hline block3 & conv7 & $3 \times 3 \times 3$ & $3 \times 3 \times 3$ & 256, stride 1 \\
\hline block4 & conv8 & $2 \times 2 \times 2$ & $3 \times 3 \times 3$ & 512, stride 2 \\
\hline block4 & conv9 & $2 \times 2 \times 2$ & $3 \times 3 \times 3$ & 512, stride 1 \\
\hline last layer & fc & $1 \times 1 \times 1$ & average pool, 512-d fc, sigmoid \\
\hline
\end{tabular}

For the network optimizer, we used Adam [118] with default parameters for momentum scheduling $\left(\beta_{1}=0.99, \beta_{2}=0.999\right)$ provided by PyTorch [119], and a mini-batch size of 128 was used for training. The learning rate started from 0.01 and was divided by 10 when the training accuracy plateaued, and the models were trained for up to 100 epochs. For our loss function, we calculated the mean square error (MSE) loss between predicted SHAPE reactivities and experimental SHAPE reactivities as

$$
\text { Loss }=\sum_{n=1}^{N}\left(P_{n}-G_{n}\right)^{2} / N
$$

where $N$ is the number of images and $P_{n}\left(G_{n}\right)$ is the predicted(experimental) SHAPE reac- 
tivity for image $n$.

\section{Output: predicting SHAPE reactivity with a CNN}

For any given 3D image that describes the surrounding environment of the considered nucleotide, our CNN model will output a real number characterizing the predicted SHAPE reactivity. This output value is confined within range from 0 to 1 .

\section{Implementation and cross-validating}

Based on the SHAPE data for 20 RNAs (totally 2455 nucleotides) collected by different experimental labs, we have 2455 SHAPE data along with the corresponding high-resolution atomic coordinates for all the nucleotides and their pertinent physical and chemical parameters. All the data together serve as the input for the $\mathrm{CNN}$. To test and validate the deep learning approach, we used the leave-one-out cross-validation method to validate the performance of our model. Each time, our model was trained on 19 RNA cases with corresponding SHAPE reactivity data and tested on 1 RNA case. This process was carried out 20 times, leaving out each RNA in turn. The overall performance is evaluated by averaging the Pearson correlation coefficients of the 20 test cases over the leave-one-out process. We also carried out this procedure for the 12 cases used to parameterize the 3DSSR function. The results of the cross-validation process are summarized in Table 3.4. The Pearson correlation coefficient was used to measure the similarity between the predicted SHAPE profile and the experimentally derived SHAPE profile. For each training and validation set in the cross-validation, we chose the model that has the best performance on the validation set to avoid overfitting. 


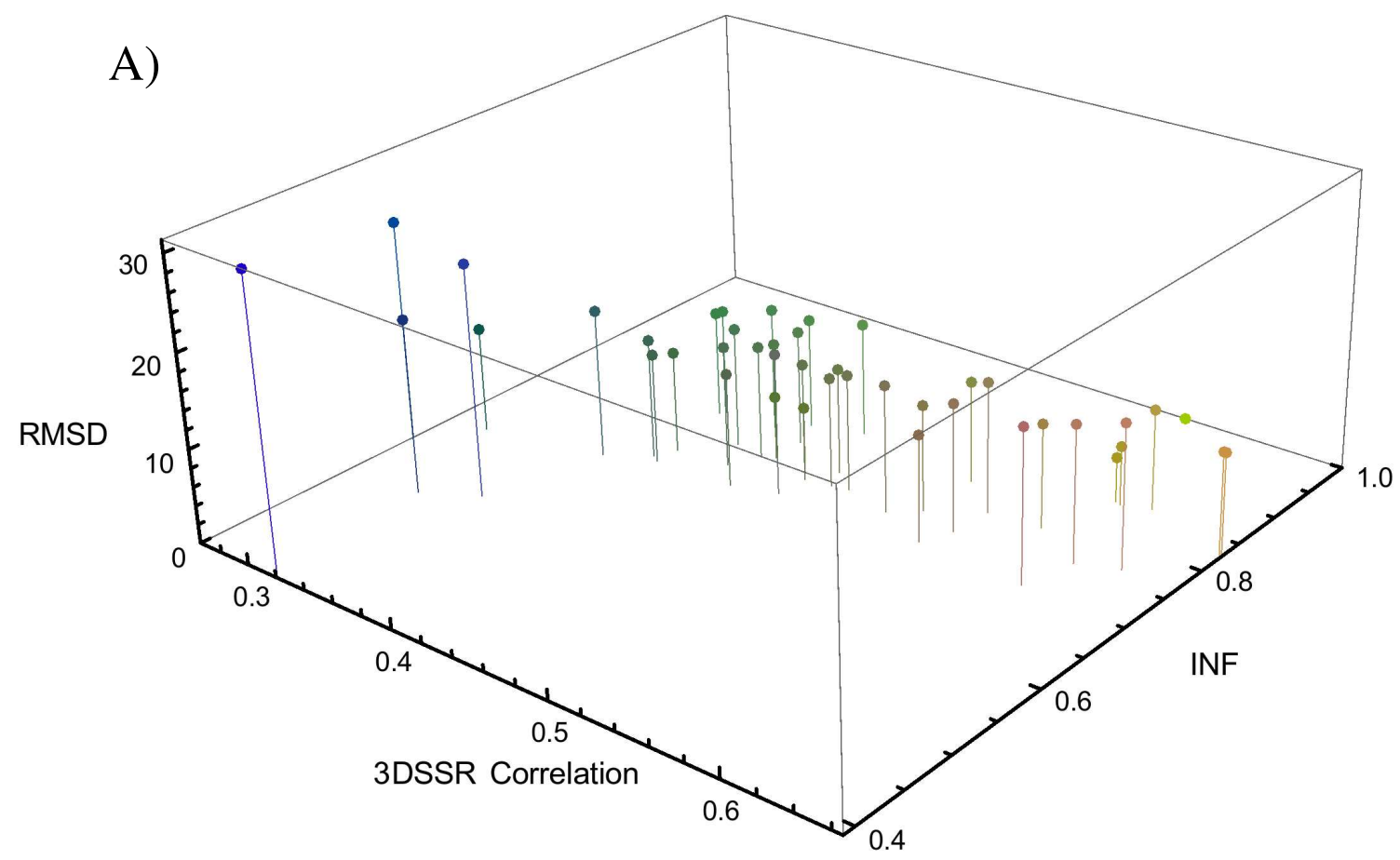

Puzzle 8 INF vs Correlation
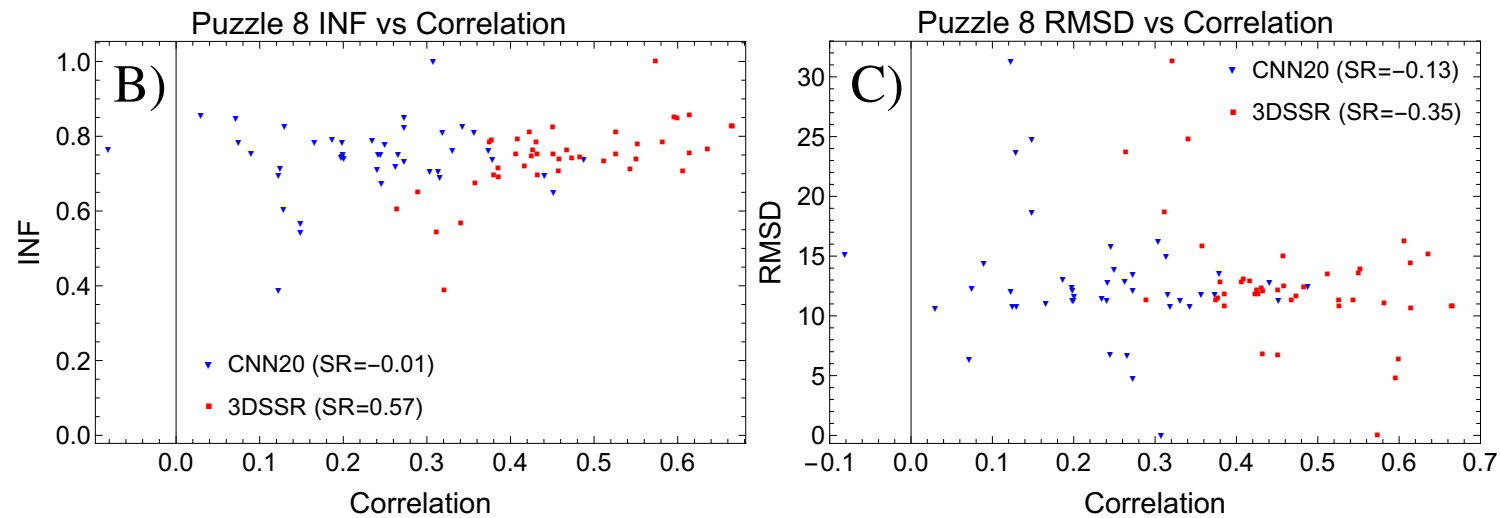

Figure 3.5: Sieving SHAPE-incompatible structures from RNA-Puzzle 8 submissions. A) The 3D representation shows the trend of the assessment results (RMSD and INF) with the correlation between 3DSSR-predicted SHAPE profiles and experimental SHAPE (3DSSR Correlation). Warmer colors indicate higher correlation, higher INF, and lower RMSD. The INF and RMSD values were taken from the RNA-Puzzles database. 2D plots of the B) INF and C) RMSD with respect to the 3DSSR and CNN20 correlations are also shown, along with their respective Spearman rank coefficients (SR). 


\subsubsection{Comparing 3DSSR to CNN models}

As can be seen in Table 3.4, the 3DSSR model generally outperforms the CNN model, regardless of whether 20 or 12 structures are used to train the CNN. In contrast, the CNN model performs substantially better on 3PDR, which may indicate that information in the structure of 3PDR leading to its SHAPE reactivity profile is contained in the other cases. Because 3PDR has high performance in the CNN model in spite of its length, we may expect improvements in other cases once the amount of training data is increased. The relatively poor performance in other cases may indicate that factors that contribute to SHAPE reactivity in those RNA are not adequately represented by the structures provided in the training set. In addition, the small fluctuations captured in the 3DSSR model by using solvated, near-native representations to fit the unknown parameters may help boost its performance over the CNN.

However, the correlation alone does not show us the discerning ability of the 3DSSR and CNN models on decoy structures. For that, we turn to the results on RNA-Puzzle 8, where the Spearman rank correlation coefficient (SR) can tell us how well the models perform on ranking the structures in comparison to objective assessments (RMSD and INF). For INF(RMSD), the SR values were 0.57(-0.35) and -0.01(-0.13) for 3DSSR and CNN20, respectively, which shows that 3DSSR markedly outperforms the CNN20 model on both ranking assessments and can be used to exclude more SHAPE-incompatible structures (see Fig. 3.5BC). 
Table 3.4: Pearson correlations between the experimental SHAPE data and the prediction algorithms: 3DSSR and the cross-validated CNN model trained on 11(19) cases and tested on the one left out, denoted as CNN12(CNN20).

\begin{tabular}{l|c|c|c|c}
\hline \hline PDB & Length (nt) & 3DSSR & CNN20 & CNN12 \\
\hline 2L8H & 29 & 0.96 & 0.85 & 0.87 \\
1AUD & 30 & 0.92 & 0.90 & 0.71 \\
2L1V & 36 & 0.83 & 0.81 & 0.79 \\
1Y26 & 71 & 0.88 & 0.52 & 0.66 \\
1VTQ & 75 & 0.71 & 0.71 & 0.80 \\
1EHZ & 76 & 0.80 & 0.77 & 0.78 \\
2GDI & 79 & 0.89 & 0.81 & 0.66 \\
3IWN & 93 & 0.74 & 0.33 & 0.38 \\
4KQY & 117 & 0.75 & 0.58 & 0.64 \\
1NBS & 154 & 0.61 & 0.48 & 0.34 \\
3PDR & 154 & 0.61 & 0.81 & 0.83 \\
3DIG & 174 & 0.70 & 0.64 & 0.68 \\
\hline Average & 92 & 0.78 & 0.68 & 0.68 \\
\hline \hline
\end{tabular}

\subsection{Conclusion}

Efficient chemical probing methods, like SHAPE, provide a wealth of information about RNA structure and dynamics. By formulating an analytical expression that captures the key factors determining SHAPE reactivity, we can predict SHAPE reactivity from individual RNA structures. After computing predictive SHAPE profiles for a set of candidate RNA 3D structures, we can sieve the structures based on the correlation between the predicted and experimental reactivities, and SHAPE-incompatible structures can be excluded. This general method of combining efficient experimental data with computational sieving may be transferred to other efficient probing methods, enabling more confident computational determination of RNA tertiary structure at lower cost.

Machine learning techniques are incapable of creating new concepts and require the training data to be a good representative of the test data. To put it simply, a dog classi- 
fication model trained with only dog images can not be used to classify cats; the model cannot be generalized to predict information it has never seen during training. Because we only have 20 RNA structures with SHAPE reactivity profiles in our data set, there is a good chance that nucleotides in a test RNA are not well represented by the other 19 structures, which results in worse performance. Additionally, using features that are important for determining SHAPE reactivity of a given nucleotide can greatly facilitate the learning process. However, finding the right combination of image channel features is not easy since the underlying mechanism that governs SHAPE reactivity is still unclear.

Although the mechanism that governs SHAPE reactivity is not fully understood, our general understanding is enough to formulate a relatively simple analytical function- the 3DSSR model - to predict reactivity based on the sensitivity of SHAPE to local nucleotide dynamics and the accessibility of SHAPE-reactive nucleotides. Because there is not enough data to apply a trained, pattern recognizing CNN to new structures, our manually constructed, analytical 3DSSR function is better at ranking structures on the basis of experimental SHAPE data. Although machine learning and advanced data-processing methods are leading to rapid advances on many problems with ample data and unclear underlying mathematical structure, physics-based models can perform better in systems where limited data is available and underlying mechanisms are known well enough to mathematically express the mechanics, even if the mechanisms are incompletely understood. As more data becomes available, we expect performance of the CNN model to improve. In the meantime, we recommend using expressions of the underlying mechanics to predict SHAPE reactivity for guiding RNA structure prediction. 


\section{Chapter 4}

\section{Sieving RNA 3D Structures with SHAPE and Evaluating Mechanisms Driving Sequence-Dependent Reactivity Bias}

This chapter was published ${ }^{1}$.

Summary: Selective 2'-hydroxyl acylation analyzed by primer extension (SHAPE) chemical probing provides local RNA flexibility information at single-nucleotide resolution. In general, SHAPE is thought of as a secondary structure (2D) technology, but we find evidence that robust tertiary structure (3D) information is contained in SHAPE data. Here we report a new model that achieves a higher correlation between SHAPE data and native RNA $3 D$ structures than the previous $3 D$ structure-SHAPE relationship model. Furthermore, we demonstrate that the new model improves our ability to discern between SHAPE-compatible and incompatible structures on model decoys. After identi-

\footnotetext{
${ }^{1}$ Travis Hurst and Shi-Jie Chen. "Sieving RNA 3D Structures with SHAPE and Evaluating Mechanisms Driving Sequence-Dependent Reactivity Bias". In: J. Phys. Chem. B 125.4 (2021), pp. 1156-1166. ISSN: 1520-6106. DOI: 10.1021/acs. jpcb.0c11365. URL: https:// doi.org/10.1021/acs . jpcb.0c11365.
} 
fying sequence-dependent bias in SHAPE experiments, we propose a mechanism driving sequence-dependent bias in SHAPE experiments, using replica-exchange umbrella sampling simulations to confirm that SHAPE sequence bias is largely explained by the stability of the unreacted SHAPE reagent in the binding pocket. Taken together, this work represents multiple practical advances in our mechanistic and predictive understanding of SHAPE technology.

\subsection{Introduction}

From design of nanotherapeutics to comprehension of fundamental mechanisms driving biological processes, the knowledge of RNA structure and function forms a cornerstone of support that we must grasp in order to prevent diseases and harness the power of RNA. In vivo, RNA molecules must adopt specific structural conformations to perform biological functions [1]. Identification of the functional structure of RNA molecules can lead to important insights into their mechanisms of action. Rapid advances in sequencing RNA molecules has led to the identification of more than 18.5 million non-coding RNA sequences in the RNAcentral (version 14) database [120]. In contrast, as of September 2020, about 5060 RNA-containing structures can be found in the RCSB protein data bank (PDB), and only 2962 of these are high resolution ( $<3.5 \AA$ ). Myriad challenges associated with experimental structure determination prevent rapid growth of the benchmark structure database [121]. To address this stark gap in knowledge, we require accurate computational prediction of RNA structures from their sequences to build our understanding of the role of RNA in biological systems. Combined with constraints from efficient experimental data, such as selective 2'-hydroxyl acylation analyzed by primer extension (SHAPE) chemical 
probing, computational models can characterize RNA structures of interest, addressing the knowledge-gap between sequence and structure information and taking full advantage of emerging chemical profiling techniques to drive structure prediction [122].

The SHAPE chemical probing method provides a single-nucleotide resolution measure of local nucleotide flexibility [27, 28]. SHAPE reagents are small ligands-e.g. 1-methyl7-nitroisatoic anhydride (1M7) - that preferentially bind to the oxygen of the 2'-hydroxyl group of RNA nucleotides in flexible regions [29]. High SHAPE reactivity for a nucleotide typically indicates that the nucleotide is located in a flexible region of the RNA, such as a flexible loop, whereas low SHAPE reactivity tends to indicate that the nucleotide is found in a rigid location, such as a helix or rigid loop [30, 32]. This efficient, solvent-based method has revolutionized 2D structure estimation for RNA [33-37, 123, 124]. Furthermore, SHAPE can be used to characterize RNA molecules in living cells $[29,125]$. Although SHAPE provides information about 2D structure, because the interactions that determine SHAPE reactivity are intrinsically 3D structure-dependent, SHAPE data contains information pertaining to the tertiary structure of RNA, making it a valuable tool for sieving 3D decoys in computational modeling. Previously, we showed that measures of local nucleotide flexibility generally correlate with SHAPE data and proposed the 3D structureSHAPE relationship (3DSSR) model to predict SHAPE reactivity from 3D RNA structure [78]. Comparing tools for predicting SHAPE from 3D RNA structure, we also showed that physics-based methods outperform pattern recognizing, machine learning techniques because of data scarcity and our ability to express the SHAPE mechanism as an analytical function of nucleotide interaction strength and structural features [79]. Combining the efficiency of SHAPE with the power of computational modeling can accelerate the search for accurate representations of RNA 3D structures. 
In this work, we identify several features of SHAPE that contribute to the underlying reactivity mechanism and use SHAPE data to expand our previous model for sieving likely 3D structure candidates from a pool of decoy structures. Because more SHAPE data is available for sequences than for 3D structures, we analyze an expanded data set to identify sequence-dependent biases in SHAPE experiments. Using replica exchange umbrella sampling (REUS) simulations, we identify a sequence-dependent mechanism that largely explains the bias previously noted in the SHAPE data [126]. With interest, we note that previous work has identified the log-normality of SHAPE data and noise [127, 128]. This implies that SHAPE reactivity directly reflects the underlying energetics of the SHAPE reaction. In comparison to the previously published 3D structure-SHAPE relationship (3DSSR) model [78], we find performance improvements of 30\%, 21\%, and $10 \%$ in average Pearson correlation (PC) of the near-native ensemble, PC of the top-ranked structure, and Spearman rank correlation (SRC), respectively. These improvements indicate that our model can accurately predict the SHAPE data from the 3D structure and reliably discern between SHAPE-compatible (near-native) and SHAPE-incompatible (non-native) structures. By investigating disparities between crystal structure information and SHAPE reactivity, we explain the discrepancies that lead to below-average prediction performance for some validation structures. The strength of this method lies in exploiting the combination of computational strength and efficient experimental data. Although SHAPE has previously been considered to provide mostly secondary structure information, we reiterate that SHAPE provides a wealth of information at the level of tertiary structure, especially for larger RNA with more long-range interactions. Furthermore, while SHAPE provides data at single-nucleotide resolution, we emphasize that interactions between a nucleotide of interest (NOI) and nearby nucleotide—and interactions between those nearby nucleotides and 
the SHAPE ligand-play an important role in estimating the reactivity of a NOI. SHAPE data is autocorrelated, and we can take advantage of the correlations within the data to extract reliable information from SHAPE profiles.

\subsection{Theory and Methods}

In this section, we describe the underlying theory underpinning the physics-based model. First, we describe the energy scoring for base pairing and stacking interactions. These combine to form the interaction energy score. We also describe how local correlations

and polarity biases in SHAPE reactivity are accounted for using a weighted averaging method. Using these energy scores and additional structural features, we provide our new estimator of SHAPE reactivity from RNA 3D structure. After that, we detail how experimental noise is factored into our performance calculations and explain improvements to the simulation and training methodology. Finally, we delineate a procedure for detecting sequence-dependent bias and provide free energy calculations from REUS to show that SHAPE sequence-dependent bias can be largely attributed to the relative stability of the unreacted SHAPE reagent in the binding pocket.

\subsubsection{Base pairing and stacking interactions}

Base pairing interactions can be categorized into 12 types, according to their nucleotide edge geometries. Using updated statistical frequencies of the base pairing interactions (see Table B.1 in Appendix B) extracted from the non-redundant RNA Basepair Catalog [129], we assigned pseudo-free energy scores to each interaction family on the basis of a quasi- 
chemical statistical potential [84]

$$
E_{\mathrm{bp}}^{(t)}(a, b)=-k_{\mathrm{B}} T \ln \left(\frac{f_{\mathrm{obs}}^{(t)}(a, b)}{f_{\exp }^{(t)}(a, b)}\right)
$$

where the expected fraction is calculated as

$$
f_{\exp }^{(t)}(a, b)=f_{\text {obs }}^{(t)} x_{a} x_{b}
$$

from the observed fraction of type- $t$ pairs $(t=1,2, \ldots, 12)$ and the mole fractions $\left(x_{a, b}\right)$ of base pairs involving $a$ and $b$ nucleotide types (A, U, G, C). The observed fractions of type- $t$ pairs between $a$ and $b$ nucleotide types $f_{\text {obs }}^{(t)}(a, b)$ are directly calculated from Table B.1 in Appendix B. The updated statistical frequencies from the non-redundant database included 20,451 entries, which is more than the 3,917 datapoints used to characterize base pairing in the previous model. Base pair types were identified in structures using RNAView [9].

If a pair of nucleotides meets the cutoffs for the distance $(d \leq D=7.202 \AA)$ between the center of the planes defined by the triads of $\mathrm{C} 2, \mathrm{C} 4$, and $\mathrm{C} 6$ atoms and angle between the planes $(\cos (\theta) \geq C=0.717)$, stacking energies are estimated for each nucleotide in the pair as

$$
\left\{\begin{array}{l}
E_{\mathrm{st}}^{(i)}(i, j)=c_{0} \cdot E_{5^{\prime}}+c_{1} \cdot E_{3^{\prime}}^{(j)} \\
E_{\mathrm{st}}^{(j)}(i, j)=c_{2} \cdot E_{5^{\prime}}+c_{3} \cdot E_{3^{\prime}}^{(j)}
\end{array}\right.
$$

where $c_{0}-c_{3}$ are weight parameters that decompose the contribution of the stacking energy from each nucleotide, $E_{5^{\prime}}$ is a parameter for the upstream $5^{\prime}$ nucleotide in a stack, and $E_{3^{\prime}}^{(j)}$ is the parameter for the downstream 3' nucleotide of type $j>i$ in a stack. This extension of the original 3DSSR model introduces sequence and $5^{\prime} \rightarrow 3^{\prime}$ polarity-dependence into the stacking energy score. See Fig. 4.1 for a visual example of base pairing and stacking 


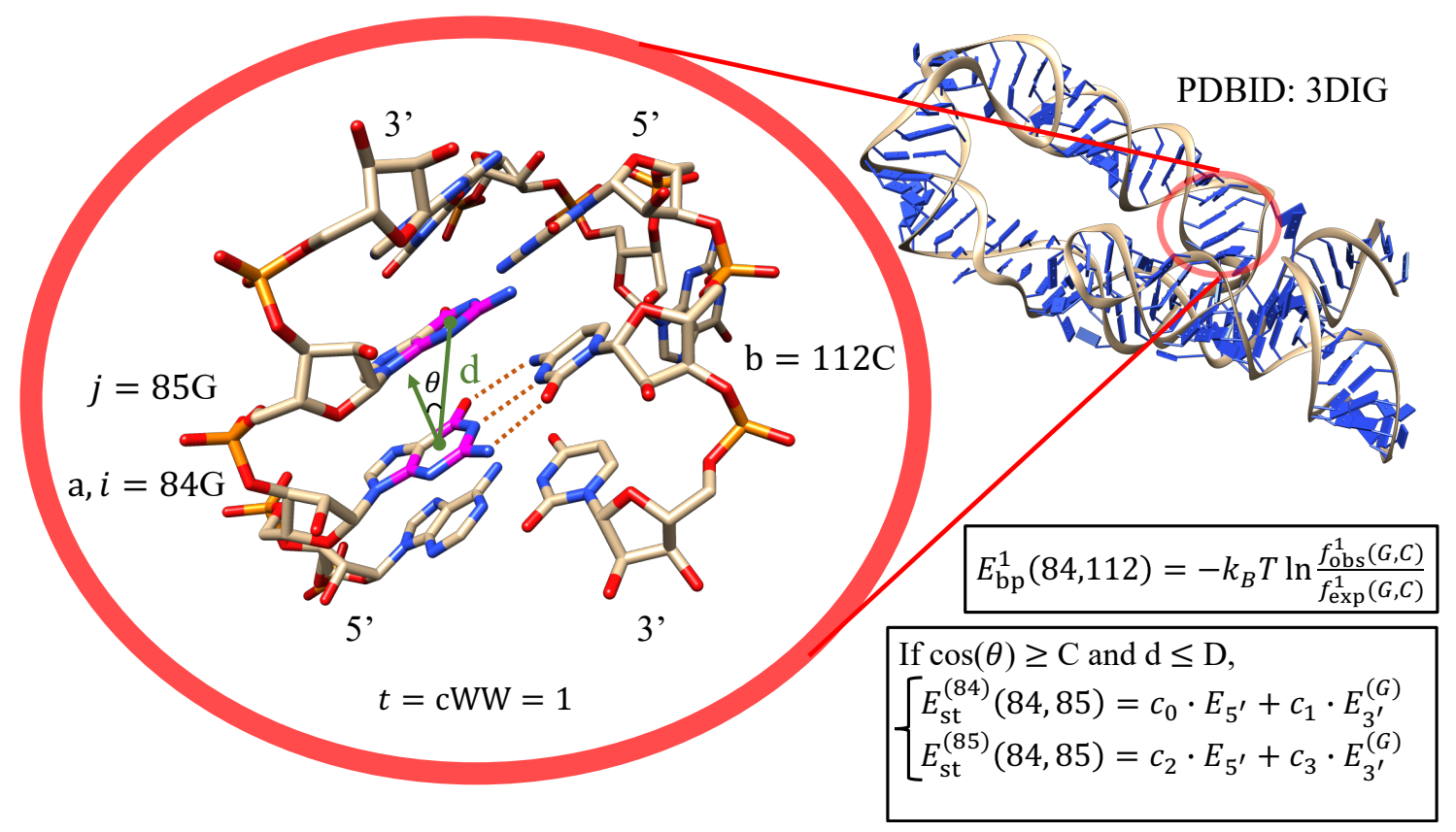

Figure 4.1: Details of stacking and base pairing calculations. To clarify the details of the stacking and base pairing calculation, we zoom into a section of a validation structure. Nucleotide $84 \mathrm{G}$ is indexed as the $a(i)$ nucleotide and is base pairing (stacking) with $112 \mathrm{C}(84 \mathrm{G})$. The $84 \mathrm{G}-112 \mathrm{C}$ base pair is a cis-Watson Crick base pair $(t=\mathrm{cWW})$. In the 84G-85G stack, the $\mathrm{C} 2, \mathrm{C} 4$, and $\mathrm{C} 6$ atoms are highlighted in magenta, and the distance (d) and angle $(\theta)$ between the planes are noted. As long as the stack meets the cutoffs described in the example calculation, we include the 3' nucleotide type-dependent stacking contributions in the energy score. 
calculations.

Pairing and stacking energies are combined into the interaction energy score (IE) for a given nucleotide $n$ as

$$
E_{\mathrm{IE}}(n)=\sum_{m}\left[A \cdot E_{\mathrm{bp}}^{(t)}(n, m)+B\right]+\sum_{k} E_{\mathrm{st}}^{(n)}(n, k)
$$

where the base pairing energy score $E_{\mathrm{bp}}^{(t)}(n, m)$ is sequence and base pairing type $t$-dependent. A free nucleotide that is next to a rigid nucleotide will be less reactive than a free nucleotide that has flexible neighbors. To account for this type of correlative effect, we further process the interaction energy score by performing weighted averaging score of the nearby nucleotides to calculate the weighted interaction energy score $\left(\bar{E}_{\mathrm{IE}}\right)$

$$
\bar{E}_{\mathrm{IE}}(i)=\frac{\sum_{j=0}^{3} w_{j} \times E_{\mathrm{IE}}(i+j-1)}{\sum_{j=0}^{3} w_{j}}
$$

where $w_{0}-w_{3}$ are weights accounting for the influence of interactions involving the nucleotide of interest (NOI) and/or neighboring nucleotides on the flexibility and SHAPE reactivity of the NOI. Constraints placed on the neighboring nucleotides by stacking or base pairing interactions will reduce the degrees of freedom and flexibility of the NOI. Because the SHAPE reagent attacks the 2'OH group, the downstream 3' nucleotide usually has more influence on the NOI than the upstream 5' nucleotide, and this polarity bias is reflected in the relative magnitudes of the weights. Furthermore, because the SHAPE reagent binding pocket is formed between the NOI and its 3 ' neighbor, the next nearest neighbor on the 3' side is also included in the score. In other words, we are evaluating the nucleotides that form the binding pocket and their neighbors in our assessment of the predicted flexibility and SHAPE reactivity of the NOI. 


\subsubsection{D structure}

In order to automate our procedure, we utilized the Dissecting the Spatial Structure of RNA (DSSR) tool to identify the 2D structure from the 3D structures [130]. This important addition to the procedure not only automates the process, it also removes human error and allows recognition of formation and breaking of base pairs during the simulations. In the previous work, the 2D structure term was 1.0 for free nucleotides and 0.01 for pairing nucleotides. Converting this concept to an energy-like score, we introduced a fit parameter $E_{2 \mathrm{D}}(n)$ to represent the energy contribution of base pairing in the 2D structure. Similar to the IE term, we performed weighted averaging on the $2 \mathrm{D}$ structures

$$
\bar{E}_{2 \mathrm{D}}(i)=\frac{\sum_{j=0}^{3} d_{j} \times E_{2 D}(i+j-1)}{\sum_{j=0}^{3} d_{j}}
$$

where $d_{0}-d_{3}$ weight the NOI and neighboring nucleotide 2D structure contributions to the NOI prediction. Nucleotides that neighbor base pairing nucleotides normally have lower SHAPE reactivity than nucleotides neighboring free nucleotides, so the weights account for these correlation effects. Again, the polarity bias in the SHAPE data is accounted for by fitting direction-dependent weights.

\subsubsection{Accounting for Other Structural Features}

We expect that accessibility of the SHAPE reagent (1M7) to the 2'-hydroxyl of a nucleotide is a necessary condition for reactivity. The free SHAPE ligand has an effective radius of about 2.0 $\AA$, so the ligand accessible surface $A_{\mathrm{SAS}}$ of the 2'-hydroxyl for each nucleotide was calculated using Visual Molecular Dynamics (VMD) [85] with a bead radius of $2.0 \AA$. 
Similar to the IE and 2D terms, we can perform weighted averaging on the $A_{\mathrm{SAS}}$ term

$$
\bar{A}_{\mathrm{SAS}}(i)=\frac{\sum_{j=0}^{3} a_{j} \times A_{\mathrm{SAS}}(i+j-1)}{\sum_{j=0}^{3} a_{j}}
$$

where $a_{0}-a_{3}$ are weights for the NOI and neighboring nucleotides to account for the influence of accessibility of nearby nucleotides on the accessibility and reactivity of the NOI. Since $A_{\mathrm{SAS}}$ is a noisy term-it can significantly fluctuate for any given nucleotide, even with the small structure changes in heavily constrained simulations - this calculation smooths the accessibility estimation, allowing the prediction to hone in on broader regions of inaccessibility that may reduce SHAPE reactivity.

In addition, previous studies found that the ribose sugar conformation is important for SHAPE-reactivity [131, 132]. Indeed, we found that addition of this structural feature into our predictive algorithm provides a marginal performance improvement. To account for this affect, we assign a fit correction $F_{\text {sug }}$ determined by the pseudorotation angle of the ribose to modulate the predicted SHAPE reactivity (see Table B.2 in Appendix B for details).

The addition of short nucleotide sequences in the tail regions during SHAPE experiments can affect the SHAPE-reactivity of those regions. Although no consensus method has been established for SHAPE, some of these tail effects can be accounted for through the addition of simple fit parameters $F_{\text {term }}$ on terminal nucleotides, so the model also accounts for those effects (see Table B.2 in Appendix B).

Bound ligands are known to reduce SHAPE reactivity for nucleotides [133]. To account for these effects, we introduced a fit ligand binding energy penalty $E_{\text {lig }}$ for 45 nucleotides that are interacting with bound ligands. 
Table 4.1: RNA structures used for validation of the model. The Protein Database ID (PDBID), length of the RNA in nucleotides (\#nt), type of RNA, and organism of origin are displayed. For RNA structures with multiple PDB entries in the database, we chose the one with the highest resolution. The SHAPE profiles for these RNA molecules are from the published experimental data [30, 36, $37,108,123]$. * Structures used in new model that were not used to validate the previous 3DSSR model [78].

\begin{tabular}{llll}
\hline PDBID & \#nt & RNA structure type & Organism \\
\hline 2L8H & 29 & TAR & HIV-1 \\
1AUD & 30 & U1A protein binding site & H. sapiens \\
2L1V & 34 & M-box riboswitch & B. subtilis \\
2K95* & 47 & P2B-P3 telomerase pseudoknot & H. sapiens \\
1Y26 & 71 & Adenine riboswitch & V. vulnificus \\
1VTQ & 74 & PreQ1 riboswitch aptamer & B. subtilis \\
1EHZ & 76 & Aspartate tRNA & Yeast \\
1P5O* & 77 & IRES domain II & HCV \\
2GDI & 78 & TPP riboswitch & E. coli \\
3MXH* & 91 & Cyclic-di-GMP riboswitch & V. cholera \\
3IWN & 93 & Cyclic-di-GMP riboswitch & V. cholera \\
4KQY & 118 & SAM-I riboswitch & B. subtilis \\
1C2X* & 120 & 5S rRNA & E. coli \\
1NBS & 149 & Ribonuclease P domain & B. subtilis \\
3PDR & 154 & M-box riboswitch & B. subtilis \\
1GID* & 158 & Group I ribozyme domain & Synthetic \\
3DIG & 174 & Lysine riboswitch & T. maritima \\
\hline
\end{tabular}




\subsubsection{Predicting SHAPE}

Several structural features can be combined into a single structure factor coefficient for each nucleotide

$$
S F_{i}=\left(\bar{A}_{\mathrm{SAS}}(i)+A_{\mathrm{SAS}}^{0}\right) * F_{\mathrm{sug}}(i) * F_{\text {term }}(i)
$$

where $A_{\text {SAS }}^{0}$ is a fit parameter accounting for RNA flexibility that may allow an apparently inaccessible nucleotide to become flexible. Similarly, the SHAPE energy score can be written

$$
S E_{i}=\bar{E}_{2 \mathrm{D}}(i)+\bar{E}_{\mathrm{IE}}(i)+E_{\mathrm{lig}}(i)
$$

where $E_{\operatorname{lig}}(i)$ is the energy-like ligand binding penalty for the $i$ th nucleotide. Combining all of these, we arrive at an estimation for SHAPE reactivity on the basis of the 3D structure

$$
p_{i}=S F_{i} * e^{S E_{i}}
$$

The form of this equation takes advantage of a Boltzmann transformation to express that SHAPE profiles are a function of the underlying energetics that drive the reaction.

\subsubsection{All-atom simulations to form near-native ensembles}

Curating starting structures from the PDB database (see Table 4.1), we performed all-atom MD simulations using Amber18 [134] with the RNA.OL3 force field. Each RNA molecule was solvated in a TIP3P truncated octahedral water box with a buffer size of $15 \AA$. Neutralizing sodium and chloride counterions were used to maintain a $1 \mathrm{M}$ sodium concentration. Coupling the system to a Langevin thermostat maintained constant temperature (300 K). Two sets of "near-native" simulations were run. In the first, only the backbone phosphate 
(P) atom positions were strongly constrained with a restraint weight of $500 \mathrm{kcal} \cdot \AA^{2} / \mathrm{mol}$ to maintain the global folding pattern. In the second set, two nucleotide atom positions in the base pairing atoms $(\mathrm{C} 2, \mathrm{C} 6)$ were also constrained. Each system was simulated in five steps using a GTX 1080 Ti NVIDIA GPU with an AMD Ryzen Threadripper 1950X 16-core, 4 $\mathrm{GHz}$ processor. In the first minimization step, only solvent atoms were allowed to move, and the RNA atoms were fixed. The full system was minimized in the second step. In the third step, the system was warmed to $300 \mathrm{~K}$ in constant volume (NVT). Fourth, the system underwent constant pressure equilibration (NPT). Finally, we ran production simulations in the NPT ensemble, writing to the Amber NETCDF file every 10 ps, which yielded 1000 snapshots of each RNA over 10 ns.

\subsubsection{Generating non-native decoy structures with coarse grained sim- ulations}

A pool of 3D decoys with native and non-native 2D structures was generated using coarse grained (CG) simulations to test the ability of the algorithm to discern between native and non-native 2D and 3D RNA structures (see Tables B.3-B.8 in Appendix B for 2D structures). Native 2D structures were extracted from PDB structures using DSSR. Low energy, non-native RNA structures were found by providing RNA sequences to Mfold [89] and taking five of the top ten results, excluding the native 2D structure. Native structure PDBs were converted into IsRNA-compatible CG structures, and CG simulations were performed using the IsRNA model in LAMMPS [135] to sample the conformational space using a temperature of $300 \mathrm{~K}$ and a Langevin thermostat. Each simulation ran for $5 \mathrm{~ns}$ with integration time-step of 0.5 fs to provide 1000 structures with RMSD from the native structure ranging from 0.5 to $14-35$, depending on RNA sequence length. These structures were 
converted back into all-atom representations and clustered with a mutual RMSD cutoff of $0.5 \AA$. Representative frames from the top 100 clusters were kept for each simulation. In total, $6 \mathrm{CG}$ simulations were performed for each of the 17 RNA molecules studied: 1 simulation using constraints provided by the native $2 \mathrm{D}$ structure and 5 simulations using constraints provided by low-energy, non-native 2D structures. The all-atom representations of the CG structures were minimized in Amber18 to remove clashes due to transforming CG representations into all-atom structures. These structures were processed identically to the Amber18-generated near-native ensembles for the remaining steps.

\subsubsection{Log-normal noise}

Multiple previous studies describe the log-normality of SHAPE data and noise [127, 128]. The form of our energy function is partially motivated by this work because log-normality of the data implies that SHAPE is a direct reflection of the underlying reaction energetics. However, using the standard Pearson correlation (PC) to compare our prediction to the SHAPE data does not account for the log-normal noise. Without loss of discerning ability, we can apply a noise term to improve our PC with SHAPE, which allows our training algorithm to focus on more severe shortcomings if a prediction for a nucleotide is close enough to the experimental reactivity. In accordance with processing methods proposed by previous authors, we scaled ("normalized") the SHAPE data by discarding fewer than $10 \%$ of outliers ( $<5 \%$ for molecules with $<100$ nucleotides) and averaging the remaining top $10 \%$ to extract the scaling factor. Because there is not yet a consensus method for SHAPE experiments, no universal scaling method that does not account for variations in the experimental methodology can perfectly scale the disparate experimental data, but hopefully, data from disparate sources will better agree after scaling than if no scaling effort was attempted. 
The previous models such as the 3DSSR model [78] did not need to attempt any scaling of the SHAPE data since a scaling factor does not affect PCs, but when including a noise function in the training process, the scaling of the SHAPE data helps maintain consistent noise approximations across different sets of experimental data.

In previous similar models such as 3DSSR [78], SHAPE data for each nucleotide was naïvely considered to be independent of its surrounding nucleotides, but looking at SHAPE data shows this to be a simplistic assumption, prima facie. Consistent with our view that SHAPE measures local flexibility at single-nucleotide resolution but that the reactivity for a NOI contains non-negligible information about the reactivity of neighboring nucleotides, we also account for the influence of neighboring nucleotides on the SHAPE reactivity for the NOI by performing weighted averaging on the experimental SHAPE reactivity, such that

$$
\bar{r}_{i}=\frac{\sum_{j=0}^{2} s_{j} \times r(i+j-1)}{\sum_{j=0}^{2} s_{j}}
$$

where $s_{0}-s_{2}$ are fit weights. Although SHAPE data has single-nucleotide resolution, this reweighting accounts for the influence of the flexibility of the surrounding nucleotides on the SHAPE reactivity for the NOI.

From previous studies [127, 128], SHAPE reactivity can be modeled as

$$
r_{i}=s_{i} e^{n_{i}}
$$

where $r_{i}, s_{i}$, and $n_{i}$ are the SHAPE reactivity for a run, ground truth SHAPE signal, and noise associated with nucleotide $i$, respectively. This equation implies that we can model 
the noise for a SHAPE experiment as

$$
n_{i}=\ln \frac{r_{i}}{s_{i}}
$$

Numerically, this equation has some problems: the noise is poorly defined when the reactivity is zero, which commonly occurs in SHAPE data, and the ground truth signal cannot be defined from a single experiment. To overcome these issues, we used the processed reactivity to estimate the noise from a numerically stable noise estimator

$$
n_{i}=\ln \left(\frac{\bar{r}_{i}}{\eta-\bar{r}}+1.0\right)
$$

where $\bar{r}$ is the average reactivity for a given SHAPE profile and $\eta \approx 12.4$ is a fit constant. The large size of $\eta$ drives down the amount of noise we use, but our noise-adjusted PCs are still substantially improved. Due to their increased flexibility, RNA molecules with higher average SHAPE reactivity will incur more noise than highly structured sequences. Adding 1.0 to the estimator sets the minimum of the noise function to 0.0 , which is desirable.

In order to compare our predictions to the SHAPE data using this noisy paradigm, we minimized the difference according to the analytically exact function

$$
\frac{d}{d f} \sum_{i}\left(f \cdot p_{i}-\bar{r}_{i}\right)^{2}=0 \Longrightarrow f=\frac{\sum_{i} p_{i} \cdot \bar{r}_{i}}{\sum_{i} p_{i}^{2}}
$$

where $p_{i}$ is the predicted reactivity for nucleotide $i$ (see Eq. 4.9), $f$ is a scaling factor for our prediction, so $p_{i}$ and $\bar{r}_{i}$ are the predicted and reweighted experimental SHAPE reactivities for nucleotide $i$. We are free to scale our prediction by a constant scaling factor without affecting the PC, but the scaling factor allows us to include noise and calculate the 
noise-adjusted PC between our prediction and SHAPE.

In calculating the noise-adjusted PC between our predicted SHAPE profile and the SHAPE data, we use the noise factor to judge the prediction accuracy. If the prediction for a nucleotide falls into the estimated noise range set by the reactivity, we say the prediction is identical to the experimental SHAPE data. If the prediction is outside of this estimated error range, we shift our prediction closer to the experimental reactivity by $n_{i}$. For the purposes of calculating the noise-adjusted PC, we take our noise-shifted prediction to be

$$
p_{i}^{\text {noise }}= \begin{cases}\bar{r}_{i} & \bar{r}_{i}-n_{i} \leq p_{i} \leq \bar{r}_{i}+n_{i} \\ p_{i} \pm n_{i} & p_{i} \gtrless \bar{r}_{i} \mp n_{i}\end{cases}
$$

Accounting for the noise in the experimental SHAPE reduces the sensitivity of our model to near-native fluctuations and improves the PC of the near-native ensemble without sacrificing any ability to discern between the near-native structures and non-native structures generated by CG simulations. Therefore, we find this procedure to be helpful in evaluating the performance of our predictive algorithm.

\subsubsection{Training the model with simulated annealing}

We greatly improved our training strategy and efficiency to optimize the ability of our algorithm to accurately select the near-native ensemble from a pool of decoy structures. The previous model (3DSSR) was trained with few parameters using less efficient random search and genetic algorithms to optimize the PC between the model prediction on the near-native ensemble and experimental SHAPE. This type of algorithm is inefficient as the parameter space grows, and it does not use information from the non-native structure 
ensemble to improve selectivity. To improve our selectivity and efficiency, we optimized a downhill simplex using simulated annealing [136]. Rather than optimizing the PC, we optimized the selectivity of the model by maximizing the Spearman rank correlation (SRC) between the Matthews correlation coefficient [137] applied to RNA tertiary structure-i.e. the interaction network fidelity (INF) [113]—and the RNA-length weighted, noise-filtered PC

$$
P C_{N}=P C \cdot \sqrt{N-1}
$$

where $N$ is the number of nucleotides in an RNA sequence. The choice of this $P C_{N}$ metric was inspired by the t-statistic. Our goal is to optimize the model on all of the available structures, but obtaining higher PCs on shorter sequences is easier, which biases the performance in favor of shorter RNA. Weighting the PC by the RNA length encourages the model to optimize the PC for structures of varying length, rather than focusing on optimizing the shorter structures. In addition, our previous model did not use information from non-native decoys to improve the discerning power of the algorithm. This training method enables us to improve the association between highly scored structures and near-native structures, as measured by the SRC, while putting reactivity data from each nucleotide on the same plane of importance, regardless of RNA length. Furthermore, rather than using all of the generated data (2,600 decoy structures for each RNA molecule), we performed random sampling of each generated structural ensemble to curate a subset of 90 structures from each of the 8 simulations (2 all-atom simulations, $6 \mathrm{CG}$ simulations) that we ran for each structure. While improving our computational efficiency, this also allows us to more uniformly sample the possible conformational space, rather than heavily weighting the near-native ensembles and skewing the SRC.

We use the leave-one-out method to train the parameters, ensuring that our parameter 
set is generalizable and not overfit. Performing 200 steps at each sampled temperature, the simulated annealing temperature schedule followed

$$
T_{n}=T_{n-1}(1-\epsilon)
$$

where $\epsilon=0.1$ and the initial temperature $T_{0}=2.0$. Note that this is not a physical temperature but a temperature relative to the SRC score that serves as an energy-like function to optimize. Termination for a training run occurred when the fractional convergence tolerance was calculated to be under 0.001. Simulated annealing runs typically converged after 15,000 steps. Descriptions of fit parameters, values for each training run, and average values can be found in Tables B.2, B.9, and B.10 in Appendix B.

\subsubsection{Sequence-dependent bias in SHAPE experiments}

While only 17 structures had SHAPE data and relatively short 3D benchmark structures available — large structures are more difficult to simulate-we wanted to assess other available SHAPE data to identify sequence-dependent bias in SHAPE experiments. To this end, we curated a set of 71 non-redundant sequences with SHAPE data (see Tables B.11-B.13 in Appendix B) from the RMDB database [108] and other work [30, 36, 37, 123]. Including sequence-dependent bias cannot improve the selectivity of the model (non-native decoys have the same sequence as the native structure), but it may lead to insights concerning the SHAPE mechanism. Previous work pointed out some bias in SHAPE experiments [126], but they found bias in the inherent reactivity of different nucleotides rather than pairwise sequence-dependent bias in SHAPE data. Here, we use a statistical potential to determine whether significant sequence-dependent bias can be found in SHAPE experiments and as- 
sign an energy-like score to these biases.

From the experimental data, we can calculate the bias towards being SHAPE-reactive as

$$
B\left(\xi, a_{i}\right)=\frac{f_{\mathrm{SHAPE}}\left(\xi, a_{i}\right)}{f\left(\xi, a_{i}\right)}
$$

where $\xi$ is a cutoff for the scaled SHAPE reactivity (nucleotides with SHAPE $\leq \xi$ ) and the reference state $f\left(\xi, a_{i}\right)$ is the fraction of each type $a_{i}=\{\mathrm{A}, \mathrm{U}, \mathrm{G}$, or $\mathrm{C}\}$ nucleotide present. The reference state is calculated using

$$
f\left(\xi, a_{i}\right)=\frac{n\left(\xi, a_{i}\right)}{N(\xi)}
$$

where $N(\xi)$ is the total number of nucleotides having SHAPE reactivity above $\xi$ and $n\left(\xi, a_{i}\right)$ is the number of nucleotides of type $a_{i}$ having SHAPE reactivity above $\xi$. This reference state removes the bias due to the prevalence of a nucleotide type in a specified reactivity range. The fractional cumulative SHAPE reactivity of a nucleotide type $a_{i}$ is expressed as

$$
f_{\mathrm{SHAPE}}\left(\xi, a_{i}\right)=\frac{\sum_{X>\xi} X\left(a_{i}\right)}{\sum_{X>\xi} \sum_{i} X\left(a_{i}\right)}
$$

where $X\left(a_{i}\right)$ is the set of SHAPE reactivities for nucleotides of type $a_{i}$. In the numerator, we sum up all the SHAPE reactivities above the cutoff for a nucleotide of type $a_{i}$, and in the denominator, we sum up all the SHAPE reactivities above the cutoff for nucleotides of all types. Scaling the SHAPE profile for each structure by its respective maximum in this calculation, the cumulation is calculated from $\xi$ to 1 . Reflecting the relative reactivity bias, an energy-like score can be calculated from this bias using

$$
E_{\text {bias }}\left(\xi, a_{i}\right)=-k_{\mathrm{B}} T \ln B\left(\xi, a_{i}\right)
$$


which can be directly compared to the relative stability of the SHAPE ligand in the binding pocket. Applying the reactivity cutoff to the NOI yields some results of interest, but more interesting results arise from looking at the effects on the SHAPE reactivity of the NOI due to increasing reactivity of neighboring nucleotides. We can extend this bias estimator to calculate the pairwise bias by replacing $a$ with $5^{\prime}-\mathrm{XY}-3^{\prime}$ ' or $5^{\prime}-\mathrm{YZ}-3^{\prime}$ pairs. If the neighboring nucleotide reactivity meets the cutoff, the NOI (Y) SHAPE reactivity is added into the calculation. This gives us a way to evaluate the impact of increasingly reactive neighbors of specific type on the SHAPE reactivity for the NOI, and this method reveals sequence-dependent pairwise bias in experimental SHAPE data.

\subsubsection{REUS to find mechanism for sequence-dependent bias}

The sequence-dependent biases in SHAPE reactivity can be largely explained by analyzing the stability of the unreacted 1M7 ligand in the binding pocket between the NOI and its downstream, 3' neighbor. The more stable the 1M7 ligand is in the binding pocket, the more opportunity it has to react. The 5' neighbor is also thought to affect the stability of this binding pocket through interactions with the NOI.

To confirm that this mechanism drives the bias seen in SHAPE experiments, we built model 5'UXYZU3' RNA 5-mers, where the XYZ sequences are the exhaustive set of A, $\mathrm{U}, \mathrm{G}, \mathrm{C}$ nucleotide combinations and terminal uracils $(\mathrm{U})$ were attached to remove bias due to terminal nucleotide force field parameters in simulations. Simulation parameter and topology files for the 1M7 ligand were created using the Antechamber module in Amber18 [134]. The 64 single-stranded 5-mers with XYZ sequences were created by pro-

ducing A-form duplex structures in the Nucleic Acid Builder (NAB) module in Amber18 and deleting the extraneous strand from the duplex. For each structure, we placed the re- 
active carbon of the 1M7 ligand near the 2'-hydroxyl of nucleotide $\mathrm{Y}$ in each 5-mer, to approximate the SHAPE-reactive conformation (see Fig. B.1 in Appendix B). In tleap, the ligand/5-mer complex was embedded in a $15.0 \AA$ truncated octahedral water box with 80 sodium ions and neutralizing chloride counterions. Minimization and equilibration was performed as detailed before: five steps were used to minimize, warm, and pressurize the system under the RNA.OL3 and GAFF force fields. After the initial equilibration, 12 replicas were created and equilibrated under their respective umbrella potentials for $100 \mathrm{ps}$. The centers of the potentials were uniformly spaced from 2.9 to $10.6 \AA$ along the reaction coordinate - the distance between the 2'-oxygen and reactive 1M7 carbon distance-with $5.5 \mathrm{kcal} / \mathrm{mol}$ spring constants. After each replica reached equilibrium, replica exchange umbrella sampling (REUS) was performed for $5 \mathrm{~ns}$ per replica (60 ns total for each 5-mer) with exchanges attempted every 50 steps $(0.1 \mathrm{ps})$.

Initial testing indicated the normal umbrella sampling simulations (without replica exchange) were more susceptible to kinetic trapping, with the system tending to get trapped between the $\mathrm{Z}$ and 3'U nucleotides for longer umbrella biasing distances (see Figs. B.2-B.3 in Appendix B). We found that REUS tends to reduce this kinetic trapping, enabling better sampling on the 5' side of nucleotide $\mathrm{Y}$ (between $\mathrm{X}$ and $\mathrm{Y}$ ) and giving us better sampling of the transition state, in addition to other advantages. The exchange acceptance rate was above 0.12 for all sampling windows, indicating sufficient umbrella overlap. Adequate umbrella overlap was also visually confirmed by viewing the overlap of the umbrella histograms (see Fig. B.4 in Appendix B). The Amber18 implementation of REUS also allows generation of high-frequency distance data because the distance data is stored at every exchange step, avoiding storage of huge numbers of frames in the trajectory while retaining a large amount of distance data. We used the pymbar python package to subsample the data 
by removing correlated data using the autocorrelation time for the distance reaction coordinate and generate reliable potentials of mean force (PMFs) from the 50,000 data points extracted from each replica. Although much of the data is discarded by pymbar, the subsampling allows us to retain more data from umbrellas with faster autocorrelation times, maximizing the efficiency of REUS and the accuracy of our PMFs. Each umbrella yielded at least 200 uncorrelated data points, with most umbrellas yielding over 1000 data points.

We calculated free energy changes from the 64 generated PMFs. First, the partition function for a state (unbound or bound) was calculated by summing over the relevant portion of the PMF, such that

$$
Z_{a \leftrightarrow b}=\sum_{i=a}^{b} e^{-U_{i} / k_{\mathrm{B}} T}
$$

where $a$ and $b$ form the minimum and maximum for the range of the state, respectively, and $U_{i}$ is the PMF energy at the $i$-th point of the reaction coordinate. To calculate the free energy for a state, we use $G=-k_{\mathrm{B}} T \ln Z$. Then, the free energy change for traveling from the unbound state to the bound state can be calculated for some XYZ 5-mer as

$$
\Delta G_{\mathrm{XYZ}}=G_{\mathrm{rX}}-G_{\mathrm{ub}}
$$

where $G_{\mathrm{rx}}$ is the free energy of the system when the ligand is bound in the reactive site and $G_{\mathrm{ub}}$ is the free energy of the system when the ligand is free and unbound. This estimation of the absolute free energy is only accurate up to a constant. To give meaning to this number, we provide a reference state - the average of our free energy changes for all XYZ ( $\Delta G_{\text {ave }}$; see Fig. B.5 in Appendix B) - that is in alignment with the spirit of the SHAPE bias analysis to calculate the relative free energy change for binding to the SHAPE-reactive 
pocket

$$
\Delta \Delta G_{\mathrm{XYZ}}=\Delta G_{\mathrm{XYZ}}-\Delta G_{\mathrm{ave}}
$$

The value of this relative free energy can be compared to the free energy estimated extracted from SHAPE-sequence bias calculations. However, we do not have enough SHAPE data to afford to spread the data across all the three-nucleotide combinations using the statistical potential from experimental data. In order to make pairwise and single nucleotide comparisons, we average the relative free energies. More details for these calculations can be found in the SM (Section B.4 in Appendix B).

\subsection{Results}

\subsubsection{Enhanced performance}

By modeling SHAPE as an energy-driven reaction affected by some local structural features, we have substantially improved selectivity and PC in comparison to the previous model (3DSSR) [78]. To compare the new model with the 3DSSR model, we used 3DSSR to predict the SHAPE reactivity for the same decoys used in validation. The new model has 38 physical parameters, and the physical description of the model makes it directly generalizable to new structures (see Table B.2 in Appendix B). Results produced here are for the left out structure of each training run. The trained parameters are highly stable, converging to nearly the same values for all of the leave-one-out runs, indicating that we have not overfit the model (see Tables B.9-B.10 in Appendix B). The Boltzmann-inspired prediction function gives us a way to directly evaluate the impact of various structure features and energy terms that drive SHAPE reactivity. Three performance metrics were used to determine 


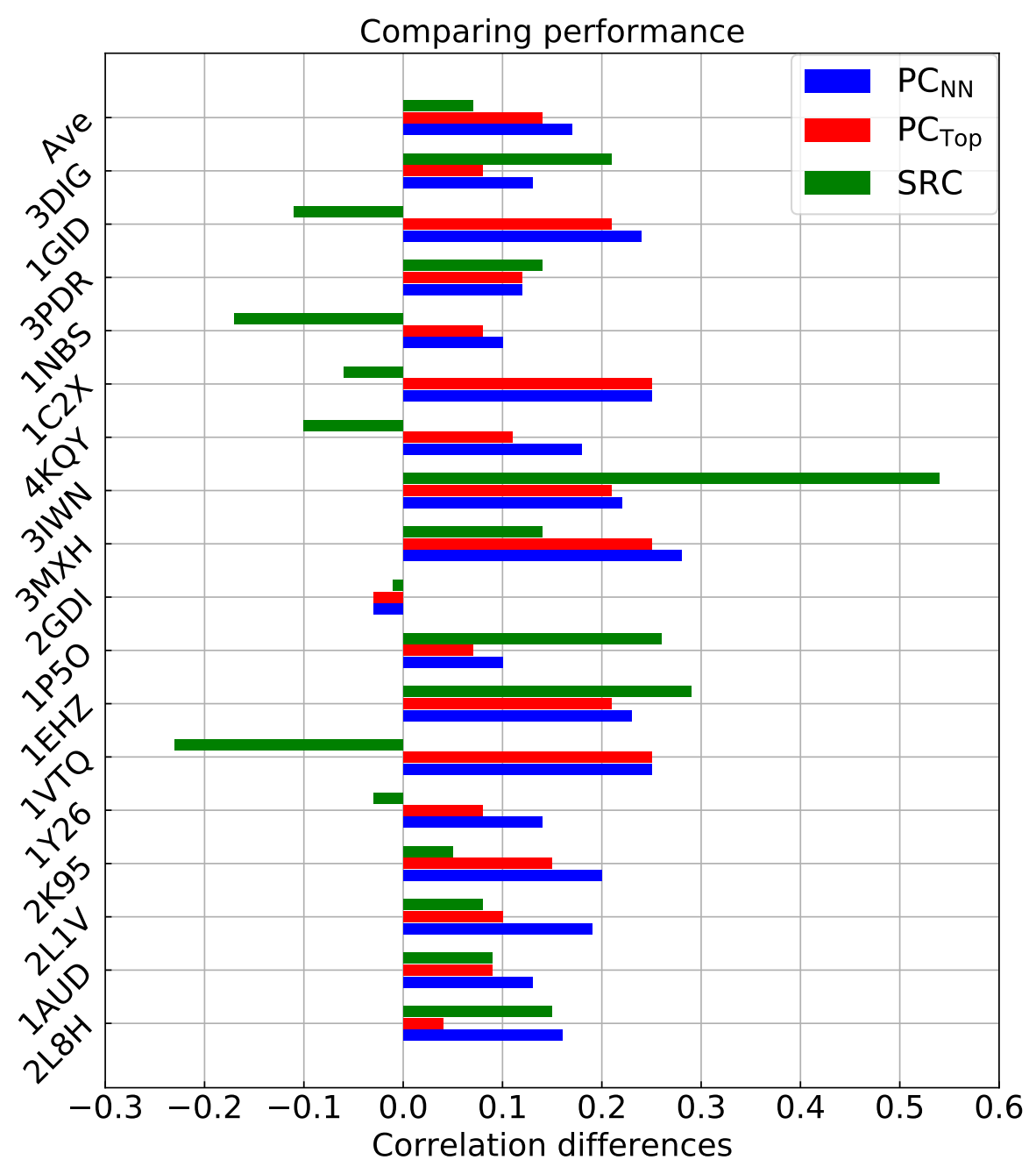

Figure 4.2: Bar chart showing performance differences. For each validation structure in leave-one-out training, the difference between the new model and 3DSSR model performance is shown. Near-native (top-ranked) structure PC performance differences are denoted 'NN' ('Top'). Average performance comparisons are at the top of the chart, denoted 'Ave'. Positive differences indicate higher performance by the new model. Absolute performance comparisons can be found in Table B.14 in Appendix B. 
the selectivity of the near-native ensembles and the agreement with experimental data (see Fig. 4.2 and Table B.14 in Appendix B). The SRC between the INF-an objective measure of structural similarity to the native structure-and the weighted Pearson correlation measures the selectivity for the near-native ensembles. On average, we have improved the SRC performance in comparison to the original 3DSSR model by $10 \%$. The average PC of the near-native ensemble and the PC of the top-ranked structure show the agreement of our model with experimental SHAPE data. The most significant advance of this model may be the $30 \%$ improvement in average PC of the near-native ensemble: this indicates that the new model is much less sensitive to small fluctuations away from the native structure, while the improved SRC shows the improved selectivity of the model (i.e. higher sensitivity to clearly non-native structures). The PC gains seen in the near-native ensembles and topranked structures emerged through optimization of SRC in training, indicating the stability of our model training across multiple measures of performance.

\subsubsection{Identifying tertiary contacts with SHAPE}

Although SHAPE has been traditionally considered a 2D technology, the RNA molecules it probes live in a $3 \mathrm{D}$ environment. To extract all of the meaningful data from SHAPE experiments, we should discard the idea that SHAPE is only useful in secondary structure prediction. To illustrate this point, we generated near-native 3D ensembles and non-native $3 \mathrm{D}$ ensembles with native and non-native 2D structures. In our longest RNA, 3DIG (see Fig. 4.1), the near-native structures occupy the highest PC range; the native 2D, non-native $3 \mathrm{D}$ structures occupy a middle regime, having higher PC than the non-native 2D structures and lower PC than the near-native structures; the non-native $2 \mathrm{D}$ structures perform the worst (see Fig. 4.3). Especially in larger structures, where the conformational space be- 
tween these ensembles is more separated, the spectrum of PCs leaves no doubt that useful 3D structure information is contained in SHAPE data. For short structures without many tertiary contacts, the effect of 3D structure on the SHAPE data is not as pronounced, but even 1P5O (77 nucleotides long) has a spectrum, showing that 3D structure information is clearly contained in the SHAPE data.

\subsubsection{The 1M7 stability in the binding pocket largely explains bias}

Although SHAPE reactivity is mostly independent of sequence identity, there are some small, sequence-dependent biases in SHAPE data. More SHAPE profiles are available with sequence information than 3D structure information, so information contained in $1 \mathrm{D}$ structure-SHAPE relationships might help improve our understanding of the SHAPE mechanism. Previous work has focused on the inherent reactivity of SHAPE with specific nucleotide types [126]. For instance, SHAPE is generally less reactive towards cytosine (C) than other nucleotide types. However, our bias calculation detailed in the Methods reveals that the sequence bias of SHAPE is deeper than simple reactivity preference. The main results from these calculations are shown in Fig. 4.4, where SHAPE sequence-dependent bias is shown to be directly correlated to the stability of the unreacted 1M7 ligand in the binding pocket of the XYZ 5-mers. While inherent reactivity differences can partially explain the SHAPE bias, the majority of this bias is driven by the stability of the SHAPE reagent in the binding pocket between the NOI and the downstream 3' nucleotide. The upstream 5' nucleotide can also affect the stability of this pocket. Calculated free energies are provided in Tables B.15-B.17 in Appendix B.

We found that these biases are too small to justify explicit inclusion in our new prediction model, especially considering that large amounts of sequence-dependence and polarity 


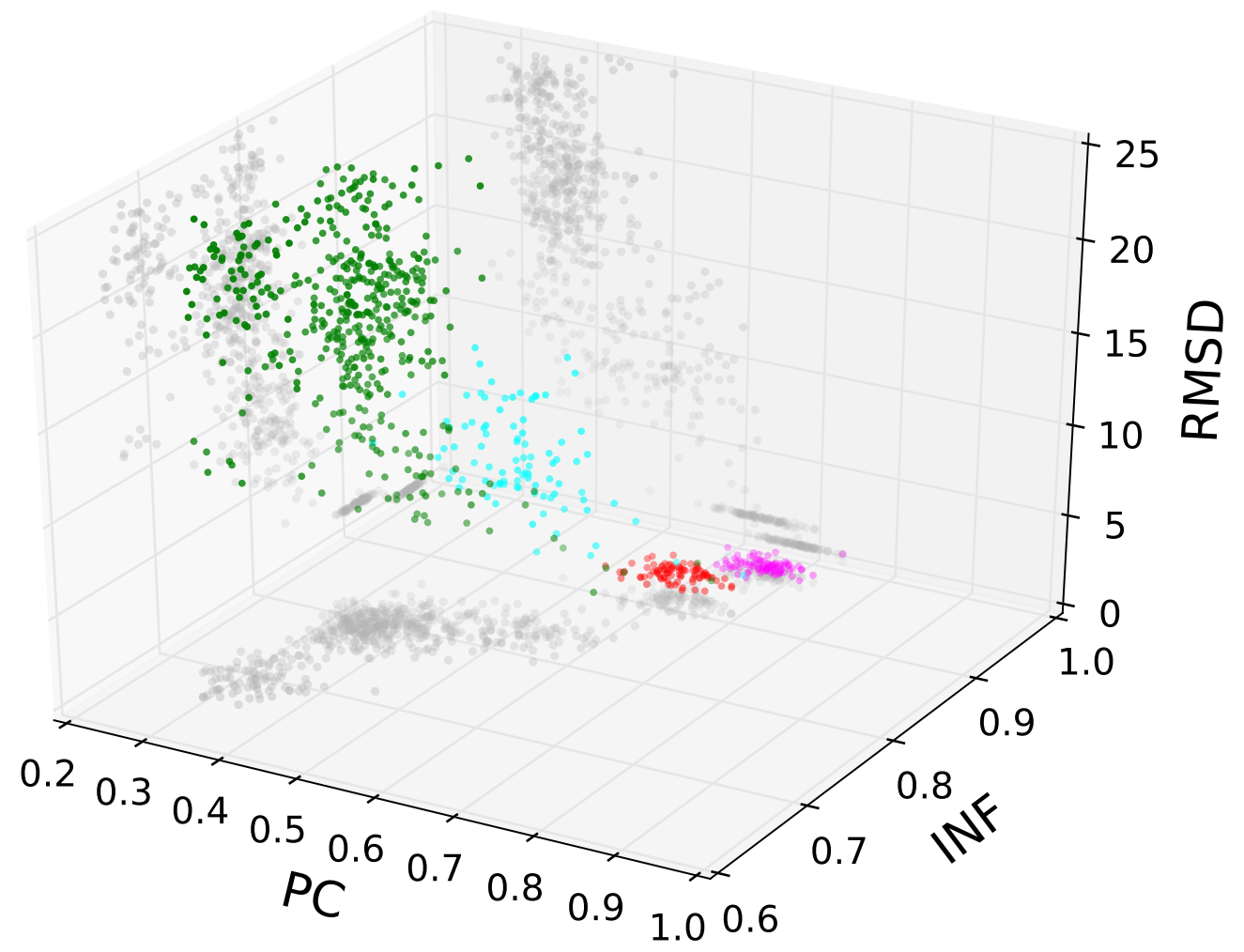

Figure 4.3: Plot showing trends between PC, INF, and RMSD for the longest validation structure, 3DIG. Each of the 90 points in magenta (red) represents a randomly selected near-native decoy from an all-atom simulation with base pairing (only backbone) restraints. Each of the 90 (450) points in cyan (green) represents a randomly selected non-native 3D (2D) decoy from a CG simulation. As the INF (RMSD) increases (decreases), the PC between SHAPE data and our prediction increases. The spectrum of results shows how our model can use SHAPE to select near-native 3D structures and indicates that SHAPE contains important tertiary structure information, especially for long structures. Shadows are projected onto surfaces for $2 \mathrm{D}$ visualization. 
bias are already included in the interaction energy (IE) calculation. For instance, we found that our stacking energy term for $\mathrm{C}$ in the 3' position is too strong to reflect only the relative

stacking energy (see $E_{3^{\prime}}^{(C)}$ in Table B.10 in Appendix B): however, this energy parameter is explained by the tendency of C to be less reactive toward SHAPE [126] and to suppress the reactivity of its neighbors. While we did not directly include information from this sequence-dependent SHAPE bias calculation in our modeling, we did find evidence that most of the sequence bias in SHAPE reactivity is driven by the stability of the unbound 1M7 ligand in the binding pocket, a mechanism that extends our understanding of SHAPE. To account for the inherent reactivity difference, we penalize nucleotides with $\mathrm{C}$ as the NOI (Y in XYZ 5-mer) by $0.4 \mathrm{kcal} / \mathrm{mol}$. Otherwise, the relative free energies shown on the X-axis of Fig. 4.4 were directly calculated from the REUS-generated PMFs.

\subsection{Discussion}

\subsubsection{Improved performance}

By incorporating an expanded perspective on SHAPE technology into our modeling, we have substantially improved the performance in terms of selectivity of the near-native structure ensemble and correlation with experimental data. The log-normality of SHAPE data and noise indicates that the SHAPE reactivity profile is a reflection of the underlying energetics driving SHAPE reactivity. Using an explicit energy-based form of the prediction function via a Boltzmann transformation has improved our selectivity as measured by SRC by $10 \%$ and the PC of the near-native ensemble to SHAPE by $30 \%$ (21\% improvement for the top-ranked structure). The PC gains are also due to incorporation of a small log-normal 
noise term that is in accordance with previous work, and this inclusion of a small amount of noise does not hamper the selectivity of the model. We also account for the autocorrelation tendencies in SHAPE data by incorporating structural and reactivity influences of neighboring nucleotides on the prediction and reactivity of the NOI. Furthermore, we better capture the sequence and directionality-dependence of SHAPE data by increasing the sophistication of our stacking calculation. Our new model is less sensitive to small fluctuations away from the native structure but more sensitive to larger $2 \mathrm{D}$ and $3 \mathrm{D}$ conformational differences than the previous model, which is desirable.

\subsubsection{Determining sequence-dependent reactivity bias mechanism}

Although there is a lack of easily analyzed 3D structures with SHAPE data, there are more sequences with SHAPE data. This provides an opportunity to evaluate sequence-dependent reactivity biases. Using a statistical potential, we found that identity of neighboring nucleotides can have a notable influence on the relative reactivity of the NOI. Performing REUS simulations, we found that most of the reactivity bias is due to the relative stability of $1 \mathrm{M} 7$ in the binding pocket between the NOI and the 3' downstream nucleotide (at the 2'-hydroxyl of the NOI). When 1M7 is more stable in the binding pocket, it will remain for longer and have more opportunity to react with the NOI. In agreement with previous work, we also saw that $\mathrm{C}$ is inherently less reactive to SHAPE, which we accounted for to more clearly show the relationship between the sequence-dependent bias and the stability of $1 \mathrm{M} 7$ in the binding pocket. 


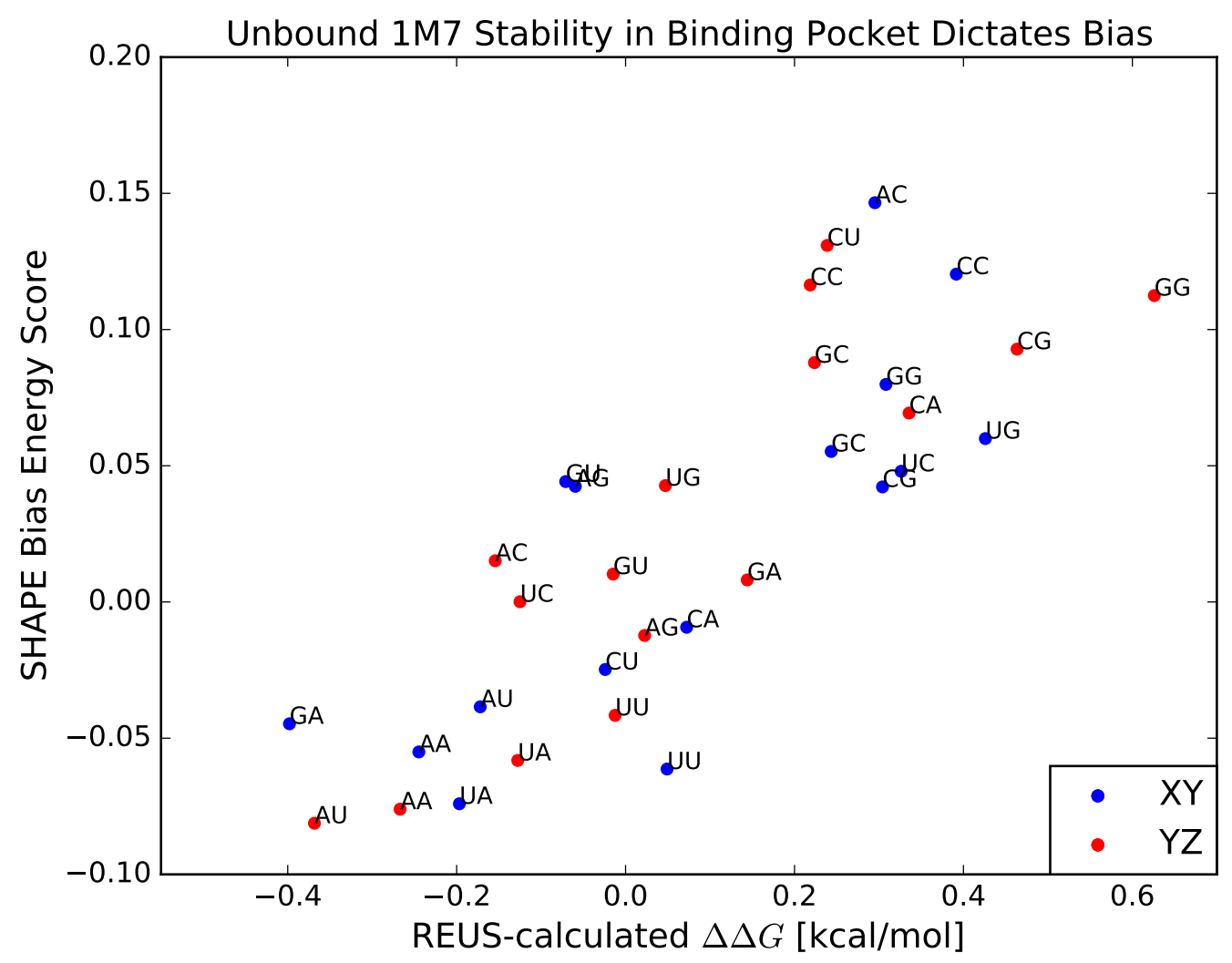

Figure 4.4: SHAPE bias energy score compared with stability of unbound ligand in binding pocket. The SHAPE bias energy score (y-axis) is directly correlated to the free energy change relative to the average calculated from REUS simulations (x-axis). The SHAPE reactivity cutoff used for the bias calculation was $\xi=0.1$. Blue (red) data points relate the pairwise bias to the REUS-calculated free energy change, influenced by the previous (next) nucleotide type, $\mathrm{X}(\mathrm{Z})$, and the NOI, Y. Inherent low reactivity of SHAPE to C is accounted for by a $0.4 \mathrm{kcal} / \mathrm{mol}$ penalty in the REUS calculation. 


\subsubsection{Detailing experimental artifacts hampering performance}

All of the validation structures with below average performance can be explained by investigating the inconsistencies between the prediction and experimental SHAPE. The major inconsistencies appear to be mainly due to crystallization or experimental SHAPE artifacts. Two of our validation structures have a best $\mathrm{PC}<0.7$, and five other structures have a best PC between 0.7 and 0.8 . Here, we detail the experimental artifacts that appear to cause the inconsistencies for these structures.

Interactions can form in crystalline structures that are not present when RNA molecules are placed in solution. For instance, the 158 nucleotide RNA with PDBID 1GID (best snap $\mathrm{PC}=0.63)$ forms a dimer in the crystal structure that is likely not present during SHAPE experiments. During the crystallization process, several nucleotides may swing out of stable stacking or base pairing conformations to make intermolecular contacts with the sister molecule. The potential structure rearrangement that would occur in solvent largely explains our poor performance on this structure, where we simply delete the extra RNA in the dimer before simulating the structure and do not account for these intermolecular interactions. Without knowing the true structure in the solution environment, it is not possible to point out every discrepancy between the crystal structure and the solution structure. However, we can point out the obvious nucleotides that form interactions in the crystal structure, and we can see that a local conformational change would explain most of the large discrepancies between our prediction and the SHAPE data. Nucleotides 152-154 in this structure all form intermolecular contacts in the dimer, and we substantially overpredict the SHAPE reactivity for all of these. Additional missed predictions for nucleotides 2, 3, and 65-71 are likely due to these effects. Some base pairing partners we think are broken in the crystallization conformational change are overpredicted, while interactions in the 5 ' tail region 
are underpredicted, indicating a significant conformational change may occur when 1GID is placed in solution. Longer range effects of this conformational rearrangement are also suspected, but we only analyze the direct effects here.

The other RNA with a best snap $\mathrm{PC}<0.7$ was $2 \mathrm{~K} 95$ (best snap $\mathrm{PC}=0.64$ ). This structure appears to have the ability to sample an alternative 2D structure in SHAPE experiments. Some nucleotides that appear to form base pairs in the PDB are reactive, and neighboring those nucleotides, unpaired nucleotides are unreactive to SHAPE. For example, nucleotides 39-40 are base paired and underpredicted, while nucleotide 41 is unpaired and overpredicted. Similarly, nucleotides 27 and 18 are paired in the structure but reactive to SHAPE. Since 2K95 is only 47 nucleotides long, a few of these 2D structure inconsistencies are hugely detrimental to the prediction performance for this structure. Without allowing for fuzziness in the SHAPE data (e.g by allowing frameshifts in the SHAPE signal by \pm 1 nucleotide, which leads to degraded selectivity performance), this kind of effect is difficult to fix without substantially altering the $2 \mathrm{D}$ and $3 \mathrm{D}$ structure of the molecule. Indeed, if we permit these frameshifts, we can obtain a PC above 0.8 for this structure. Since we are not comfortable with sacrificing our selectivity or tweaking the benchmark structure to better match the SHAPE data, we live with the below average performance for this structure.

For the other six validation structures with below average performance, a variety of explanations can be found. While performance for $3 \mathrm{MXH}$ (best $\mathrm{PC}=0.73$ ) has been substantially improved in comparison to 3DSSR (best PC $=0.57$ ) by accounting for ligand interactions, it remains below average due to protein-RNA interactions in the crystal structure. The SHAPE experiments were probably performed in absence of the protein, leading to the 10 nucleotide loop forming a more stable, rigid structure. Even if the protein was 
present in the SHAPE experiments, the model does not consider RNA-protein interactions, leading to below average performance for $3 \mathrm{MXH}$. In the crystal structure, the loop is extended due to these intermolecular interactions, and we do not permit the loop to sample more stable conformations in the simulations. Therefore, our model overpredicts the reactivity of these nucleotides. Although 1 NBS (best $\mathrm{PC}=0.75$ ) is improved in comparison to the old model (best $\mathrm{PC}=0.57$ ), performance on this structure remains below average because we do not restrain any stacking interactions in the simulations, and the structure is pretty long (149 nucleotides). Restraining base stacking for several nucleotides could improve the performance on the near-native ensemble, but we do not think that is necessary due to the decent correlation we already obtained from this long structure. The 4KQY riboswitch (best PC $=0.72$ ) is susceptible to conformational switching in SHAPE that we cannot easily account for by viewing the PDB structure. Several base pairing interactions are absent in the crystal structure that would improve the performance for this structure if enforced in simulations. However, as we mentioned, adjusting the crystal structure to better match the SHAPE data is not a tenable approach for us. Two of these underperforming structures-1C2X (best PC $=0.71$ ) and 1P5O (best PC $=0.7$ ) - have noisy SHAPE data, which may indicate that the structures are better represented by a broader structural ensemble in solution, rather than the tightly constrained near-native ensemble we provide to the model. In order for the 3D PDB structure to match the SHAPE data, large regions of rigidity are helpful, which $1 \mathrm{C} 2 \mathrm{X}$ lacks. On the other hand, the 1P5O 3D structure has several base pairing regions that are reactive to SHAPE, indicating that fraying may be a problem in the SHAPE experiment for this structure. The 174 nucleotide structure 3DIG (best PC = 0.74) is the longest structure in our dataset, and the below average PC performance for this structure is due to the large size. 


\subsection{Conclusion}

By including autocorrelation effects, log-normal noise, and sequence-dependence in the energy-based SHAPE prediction, we substantially improved our ability to predict SHAPE data from RNA 3D structure. Furthermore, we showed that stability of the unreacted SHAPE reagent in the binding pocket contributes to sequence-dependent reactivity bias in SHAPE experiments. While SHAPE has single-nucleotide resolution, it also has clear autocorrelation effects relating to the character and rigidity of neighboring nucleotides that were not previously considered. The influence of neighboring nucleotides is a small but significant aspect of SHAPE reactivity. Constraints on neighboring nucleotides reduce the conformational propensity and the SHAPE reactivity of the NOI. The log-normality of SHAPE data indicates that SHAPE reactivity profiles may be viewed as a direct expression of the energetics of the underlying reaction mechanism. Accounting for noise in SHAPE experiments leads to improved performance by removing sensitivity to small fluctuations from the native structure that should be present in solution experiments. To explore the mechanism driving sequence-dependent bias in SHAPE experiments, we performed exhaustive REUS simulations on RNA 5-mers, and free energies extracted from these simulations trend with statistical energy estimates of sequence-dependent reactivity bias, supporting the idea that bias in SHAPE reactivity is driven by the stability of the SHAPE reagent in the binding pocket formed between the NOI and the downstream 3' neighbor.

Our model uses efficient SHAPE data to drive RNA 3D structure prediction. The concepts espoused in our model may be transferred to data from other chemical probing experiments to more efficiently predict 3D structures. The knowledge gap between RNA sequence and structure can be reduced by combining computational modeling with prob- 
ing experiments. In lieu of experimental input, the rugged free-energy landscape of RNA makes predicting functional, native structures difficult. Our model works to overcome this difficulty by sieving SHAPE-compatible, near-native, 3D structure candidates from a pool of decoys, reducing the search space on the basis of efficient SHAPE data. 


\section{Chapter 5}

\section{A Bayes-inspired theory for optimally building an efficient coarse-grained folding force field}

This chapter was published ${ }^{1}$.

Summary: Because of their potential utility in predicting conformational changes and assessing folding dynamics, coarse-grained (CG) RNA folding models are appealing for rapid characterization of RNA molecules. Previously, we reported the iterative simulated RNA reference state (ISRNA) method for parameterizing a CG force field for RNA folding, which consecutively updates the simulation force field to reflect marginal distributions of folding coordinates in the structure database and extract various energy terms. While the IsRNA model was validated by showing close agreement between the IsRNA-simulated and experimentally observed distributions, here, we expand our theoretical understanding of

\footnotetext{
${ }^{1}$ Travis Hurst, Dong Zhang, Yuanzhe Zhou, and Shi-Jie Chen. "A Bayes-inspired theory for optimally building an efficient coarse-grained folding force field". In: Commun. Inf. Syst. 21.1 (2021), pp. 65-83. DOI: https://dx.doi.org/10.4310/CIS.2021.v21.n1.a4.
} 
the model and, in doing so, improve the parameterization process to optimize the subset of included folding coordinates, which leads to accelerated simulations. Using statistical mechanical theory, we analyze the underlying, Bayesian concept that drives parameterization of the energy function, providing a general method for developing predictive, knowledgebased, polymer force fields on the basis of limited data. Furthermore, we propose an optimal parameterization procedure, based on the principal of maximum entropy.

\subsection{Introduction}

The dynamics and structure of biological polymers, such as ribonucleic acid (RNA), provide insight into their evolutionary functional mechanics [138]. To grasp the function of existing RNA molecules and to design RNA-based therapeutics, we require deep knowledge of tertiary (3D) structure, kinetics, and thermodynamic stability that can be gained through rapid computational characterization techniques [139]. Recent discoveries highlighting the diversity and biological importance of RNA molecules have spurred rapid advancements in structure prediction $[16,17,20,140,141]$, which use diverse methods, from bioinformatics data fitting to all-atom simulations, to predict RNA structure [142]. Knowledge-based, template-fitting methods can rapidly predict RNA structures with phylogenetic similarities to existing structures, especially if templates are available for the structure of interest [47]. However, they are limited by the availability of known structures and fragments, and most of these predictive methods do not attempt to predict conformational changes or folding dynamics, limiting their usefulness for predicting RNA characteristics. Unlike the templatebased methods, all-atom molecular dynamics (MD) simulations do not necessarily rely on existing templates because of their ab initio nature, and they can be used to detail folding 
dynamics [48-50]. However, for lengthy RNA ( $>40$ nucleotides), the enormous conformational space makes exhaustive, all-atom MD on the scale of milliseconds or seconds impossible with current computational limitations [51, 143].

Taking advantage of the strengths of both template-based, data fitting approaches and physics-based methods, coarse-grained (CG) molecular dynamics (CGMD) simulations constitute an appealing middle-ground between these extremes. By representing groups of atoms with CG pseudobeads, we overcome the limitations of the intrinsically large conformational space of long RNA molecules, smoothing the all-atom, rugged free energy landscape and selecting only the degrees of freedom necessary to capture the conformational dynamics, which accelerates simulations by both reducing the computed degrees of freedom and increasing the diffusion coefficient of the molecule on the energy landscape [52]. An ideal CG folding model would use a simple description that optimizes sampling efficiency while extending the ability to accurately estimate observables to larger RNA systems. CG force fields can be parameterized using physics-based, knowledgebased, or hybrid methods. Physics-based methods generally use clever averaging schemes to coarsely estimate forces from all-atom interactions, while knowledge-based methods calculate interaction occurrence frequencies from solved 3D structures. Hybrid approaches may use knowledge of the solved structures to estimate local, physical potentials from statistical distributions-such as bond stretching, bond angle bending, and torsion angle constraints-or non-local energy functions may be calculated using physics-based methods [144, 145]. Regardless of the particular parameterization strategy, CG folding methods can overcome computational limitations seen in all-atom sampling while providing more information about conformational dynamics than static, template-based prediction methods. 
Previously, we reported the IsRNA method for parameterizing a CG force field for RNA folding [53]. While diverse CG models using a variety of resolutions and representations have been developed [26, 47, 140, 144-152], the IsRNA model represents nucleotides using four or five beads and has two main advantages. Firstly, the IsRNA model estimates an $n$-dimensional joint probability distribution from limited experimental data, using iterative, consecutive inclusion of observed folding coordinate distributions, which allows the model to account for correlation effects, where $n$ is the number of variables required for convergence between the simulated and experimentally observed marginal distributions of the set of considered folding coordinates. Secondly, the IsRNA model accounts for both native and non-native interactions in RNA folding. Provided with 2D constraints, the force field reproduces RNA observables and known 3D structures with alacrity. In combination with efficient experimental data to determine the 2D structure, this method can rapidly fold RNA molecules, finding low-energy candidates that represent the native structure. Efficient data can also be used to sieve experimentally-compatible structures, strengthening the prediction quality [78].

Here, we lay out a cohesive theory for parameterizing a CG force field for predictive polymer folding on the basis of knowledge extracted from a structural database. Using an iterative, Bayes-inspired approach, we construct a CG force field from consecutive simulations and marginal distributions of collective variables extracted from the RNA PDB database. The distributions of these variables are not statistically independent, so their joint distribution cannot be calculated by simply multiplying their marginal distributions together. Furthermore, the data is limited, so a joint distribution that reflects the full system energetics cannot be directly extracted from the structural data. Since the marginal distributions are not independent and we do not have enough data to extract an accurate 
force field from a joint distribution of folding coordinates, we combine data from reference simulations with observed marginal distributions to update our prior estimate of the joint force field in a Bayes-inspired approach. By adding new marginal distributions into the model one-by-one until all of the considered marginal distributions are reproduced by the energy function, we estimate a joint energy function for CG polymer folding simulations. This paper uses statistical mechanics to clarify the underpinning theory and assumptions in the model. Parameterization of the force field is optimized using the principle of maximum entropy, which informs the model of the optimal marginal distribution to include in the following step. In previous work, the principle of maximum entropy has been used for modeling intrinsically disordered proteins [153] and for combining experimental and simulation data [154]. We show that the principle of maximum entropy is directly related to the model, providing support for the underlying theory. Furthermore, the principle of maximum entropy has a Bayesian interpretation [155], providing a conceptual framework for easily understanding the model theory. We also illustrate the predictive power of the IsRNA model by applying it to accurately fold RNA into near-native 3D structures.

\subsection{Theory and Methods}

In this section, we describe and expand the theory supporting the iterative simulated reference state method of building a CG polymer force field, showing how to optimize inclusion of new folding coordinates consistent with the principle of maximum entropy. First, we describe the system setup and provide a simple example for how, in theory, we can use statistical mechanics principles to extract a CG energy function that is consistent with observations in the structure database. Second, we generalize and expand this theory into 
a workable model that uses iterative corrections to avoid growth of numerical instabilities during the practical parameterization process for a general polymer. Finally, we describe the principle of maximum entropy and show how it can be used to optimize the order of inclusion of new folding coordinates. We also show that our procedure is consistent with the principle of maximum entropy to provide further evidence that the method for parameterizing a $\mathrm{CG}$ polymer energy function is sound.
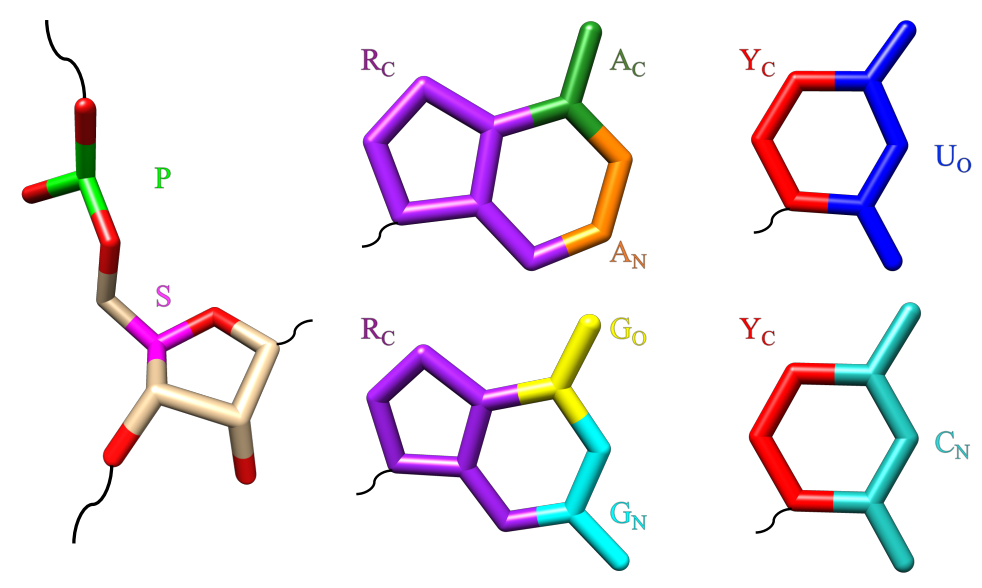

Figure 5.1: Coarse-grained descriptions of canonical A, U, G, C nucleotides. The backbone is represented by beads located at the $\mathrm{P}$ and $\mathrm{C} 4$ ' atoms (denoted $\mathrm{S}$ for the related ribose sugar). Purine (A and $G$ ) bases are represented by three beads each $\left(R_{C}, A_{C}, A_{N}\right)$ and $\left(R_{C}, G_{O}, G_{N}\right)$. Pyrimidine $(\mathrm{U}$ and $\mathrm{C})$ bases are represented by two beads each $\left(\mathrm{Y}_{\mathrm{C}}, \mathrm{U}_{\mathrm{O}}\right)$ and $\left(\mathrm{Y}_{\mathrm{C}}, \mathrm{C}_{\mathrm{N}}\right)$. CG beads for the bases are centered at the corresponding heavy-atom group center-of-mass.

\subsubsection{Setting up the system}

In the IsRNA model, we use two CG beads ( $\mathrm{P}$ and $\mathrm{S}$ ) to represent the phosphate and sugar moiety on the backbone, respectively. For the base, purines (pyrimidines) have three (two) CG beads, and the model uses a total of ten beads. Non-local, base pairing interactions are realized with two pairwise interactions, so we can model hydrogen bonding configurations and energies for various base pairing configurations. See Fig. 5.1 for details of the mapping 
from all-atom structure to $\mathrm{CG}$ representation.

We begin our approach with the most basic assumptions about RNA polymer interactions. Namely, we assume a non-interacting reference state and extract statistical potentials of bond stretching connectivity and Lennard-Jones (LJ) volume exclusion terms so that the backbone energy is

$$
E_{\mathrm{bk}}(b, r)=E_{\mathrm{bond}}(b)+E_{\mathrm{LJ}}(r)
$$

and we use those as the starting CG force field, generating the $\operatorname{ref}_{0}$ conformational ensemble. As is shown in Fig 5.2A, this information is sufficient to reproduce the $\theta_{2}$ bond angle distribution through simulation, which is already determined by the chain connectivity and volume exclusion information.

Our goal is to accurately estimate the folding energy function for RNA interactions, which are captured by bond distance $b$, bond angle $\theta$, torsion $\phi$, and distance-based $r$ nonlocal base interaction energies. In summary, our total energy function can be written as

$$
E_{\mathrm{tot}}(b, r, \theta, \phi)=E_{\mathrm{bk}}(b, r)+E_{\text {angle }}(\theta)+E_{\text {torsion }}(\phi)+E_{\text {pair }}(r)+E_{\text {ele }}(r)
$$

Although this is written as a sum of energy functions, the correlations are implicitly included through simulations, which allows us to ascertain what information about a new folding coordinate is already included in the estimated CG force field.

\subsubsection{Supporting the IsRNA theory with statistical mechanics}

Here, we provide an example to illustrate how we can use statistical mechanics to build the total force field by consecutively updating our prior energy function to enforce compatibility with an observed folding coordinate distribution. Using $E_{\mathrm{ref}}^{(i)}$ as the reference state 
force field from inclusion of the $i$ th folding coordinate, we can run a CGMD simulation and extract the simulated distribution $p_{\text {ref }}\left(\theta_{j}\right)=\left.p_{\text {sim }}\left(\theta_{j}\right)\right|_{\text {ref }}$ of the $j$ th bond angle $\theta_{j}$. From the PDB database, we also know the observed distribution $p_{\mathrm{obs}}\left(\theta_{j}\right)$, and we can calculate the difference between the observed and reference energy functions using

$$
E\left(\theta_{j}\right)=-\ln p_{\text {obs }}\left(\theta_{j}\right)+\ln p_{\text {ref }}\left(\theta_{j}\right)=-\ln \frac{p_{\text {obs }}\left(\theta_{j}\right)}{p_{\text {ref }}\left(\theta_{j}\right)}
$$

where we use the dimensionless $k_{\mathrm{B}} T=1$ in this section for simplicity. Accounting for the difference between the observed distribution and the CGMD-generated reference distribution, we define the energy function for the next simulation as

$$
E_{\mathrm{ref}}^{(i+1)}\left(\theta_{j}\right) \equiv E_{\mathrm{ref}}^{(i)}\left(\theta_{j}\right)+E\left(\theta_{j}\right)
$$

Physically, this ensures that our energy function is only adding the missing information about $\theta_{j}$ into the force field. Explicitly, we can show that this energy function will reproduce the observed distribution. The marginal reference distribution of $\theta_{j}$ generated via simulation can be written in terms of the reference joint energy function with previously added terms integrated out:

$$
p_{\mathrm{ref}}^{(i)}\left(\theta_{j}\right)=\int e^{-E_{\mathrm{ref}}^{(i)}(b, r, \theta, \phi)} d b d \phi d r d \theta_{0} \ldots d \theta_{j-1}
$$

Similarly, the simulated distribution of $\theta$ after including information about the observed 
distribution can be written

$$
\begin{aligned}
p_{\mathrm{sim}}^{(i+1)}\left(\theta_{j}\right) & =\int e^{-E_{\mathrm{ref}}^{(i+1)}(b, r, \theta, \phi)} d b d \phi d r d \theta_{0} \ldots d \theta_{j-1} \\
& =e^{-E_{\mathrm{ref}}^{(i)}\left(\theta_{j}\right)} e^{-E\left(\theta_{j}\right)} \\
& =p_{\mathrm{ref}}^{(i)}\left(\theta_{j}\right) \frac{p_{\mathrm{obs}}\left(\theta_{j}\right)}{p_{\mathrm{ref}}^{(i)}\left(\theta_{j}\right)}=p_{\mathrm{obs}}\left(\theta_{j}\right)
\end{aligned}
$$

which shows that simulations using $E_{\mathrm{ref}}^{(i+1)}(b, r, \theta, \phi)$ as the CGMD force field can reproduce the marginal probability for some bond angle $\theta_{j}$ after information about that coordinate is included in the force field. Indeed, once we include a coordinate, we find that the simulated distribution does match the observed distribution for that folding coordinate (see Fig. 5.2A-D).

To emphasize our previous point, although the force field is an additive sum of the different terms, the local variables $(b, \theta, \phi)$ are still correlated through the non-local distance ( $r)$, which is a function of $(b, \theta, \phi)$ in a highly coupled form. Similarly, the $E(\theta)$ and $E(\phi)$ energy terms are also coupled through non-local interactions. Without inclusion of the non-local terms, some of the local terms may be physically uncorrelated.

\subsubsection{Generalizing and numerically stabilizing the model}

Up until here, we have simplified the theoretical arguments in the previous work [53]. Now, we will pivot to expand the IsRNA approach in greater theoretical and practical detail to provide a general method for parameterizing a CG polymer force field. In general, the joint probability distribution cannot be uniquely determined from the set of marginal probability distributions that compose it, which is why we cannot simply multiply together all of the observed marginal distributions we find in the structure database to calculate the joint 

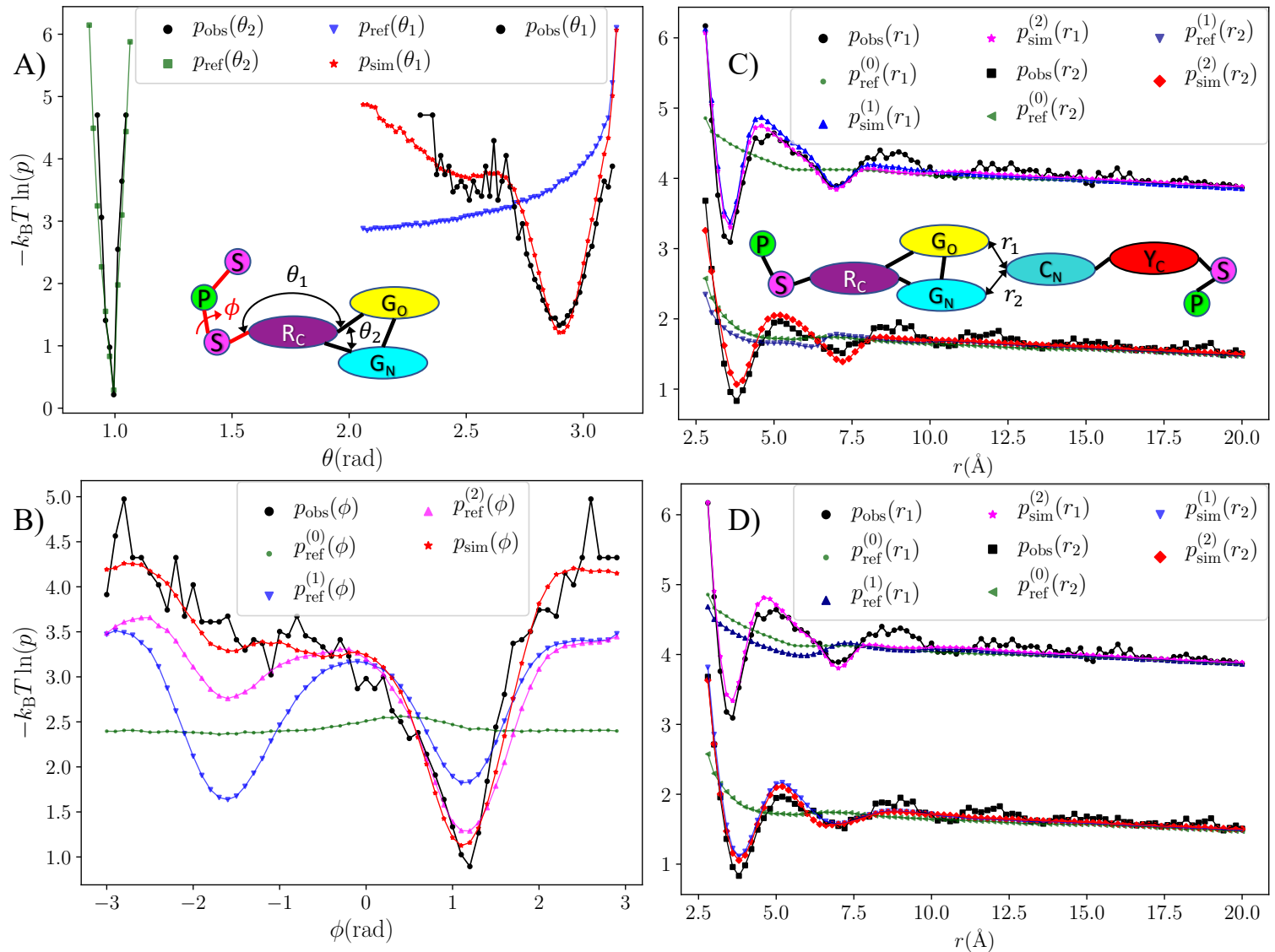

Figure 5.2: Correlated folding coordinates lead to same final energies regardless of parameterization order. A) After incorporating bond length information in our reference force field, information about the $S-R_{C}-G_{O}$ bond angle $\theta_{1}$ still needs to be included because the reference simulation does not capture the $\theta_{1}$ distribution. However, a correlated $G_{O}-R_{C}-G_{O}$ angle $\theta_{2}$ is fully captured by the reference force field without being explicitly included in the force field. B) As we include more folding coordinates in our energy function, the 5 'S-P-S- $\mathrm{R}_{\mathrm{C}} 3^{\prime}$ torsion angle $\phi$ becomes more similar to the observed distribution, indicating correlation between $\phi$ and the previously included structure factors. C) and D) Although the individual factors are correlated, the final energy function yields the same final energies, regardless of parameterization order. In C), the $r_{1}$ folding coordinate is incorporated into the force field, followed by $r_{2}$. In D), the parameterization order is reversed, but the final total energies and distributions produced by simulation are unchanged. 
distribution and the total force field. However, by simulating the conditional probability distributions, we may estimate the joint probability distribution. In theory, we can broaden our method to use any collective variables of the system, and we are not limited to simple folding coordinates, such as distances and angles. However, the more time-consuming a collective variable is to calculate, the less efficient simulations using that variable will be, so simple distances and angles are appealing building blocks for a CG force field. We take the general perspective that the marginal distribution of a collective variable extracted from a reference simulation as described in the previous section can be viewed as the marginal distribution, conditional on the previously included distributions of collective variables. Then, the estimated energy function after each consecutive step is extracted from the estimated joint distribution of all the considered collective variables, conditional on the already included distributions of collective variables.

Our energy function is considered to converge when the estimated joint distribution after a step is sufficient to reproduce via simulation all of the considered observed marginal distributions, including those marginal distributions that have not been explicitly included in the joint distribution. For instance, assume we have a set of $m$ calculated collective variables $\left(x_{0}, \ldots, x_{m}\right)$. Assume that so far in our procedure, we have explicitly included $j(<m)$ collective variables to produce an energy function $E\left(x_{0}, \ldots, x_{j}\right)$. If the marginal distributions observed from the structural database agree with the marginal distributions extracted from the simulation results, the energy function converged, and our force field is adequately parameterized, having captured all of the available information in the considered distributions. While here we use marginal probability distributions, the explicit inclusion of some correlation effects by consecutively including some joint distributions of pairs of collective variables may be beneficial to explore, if enough data is available. 


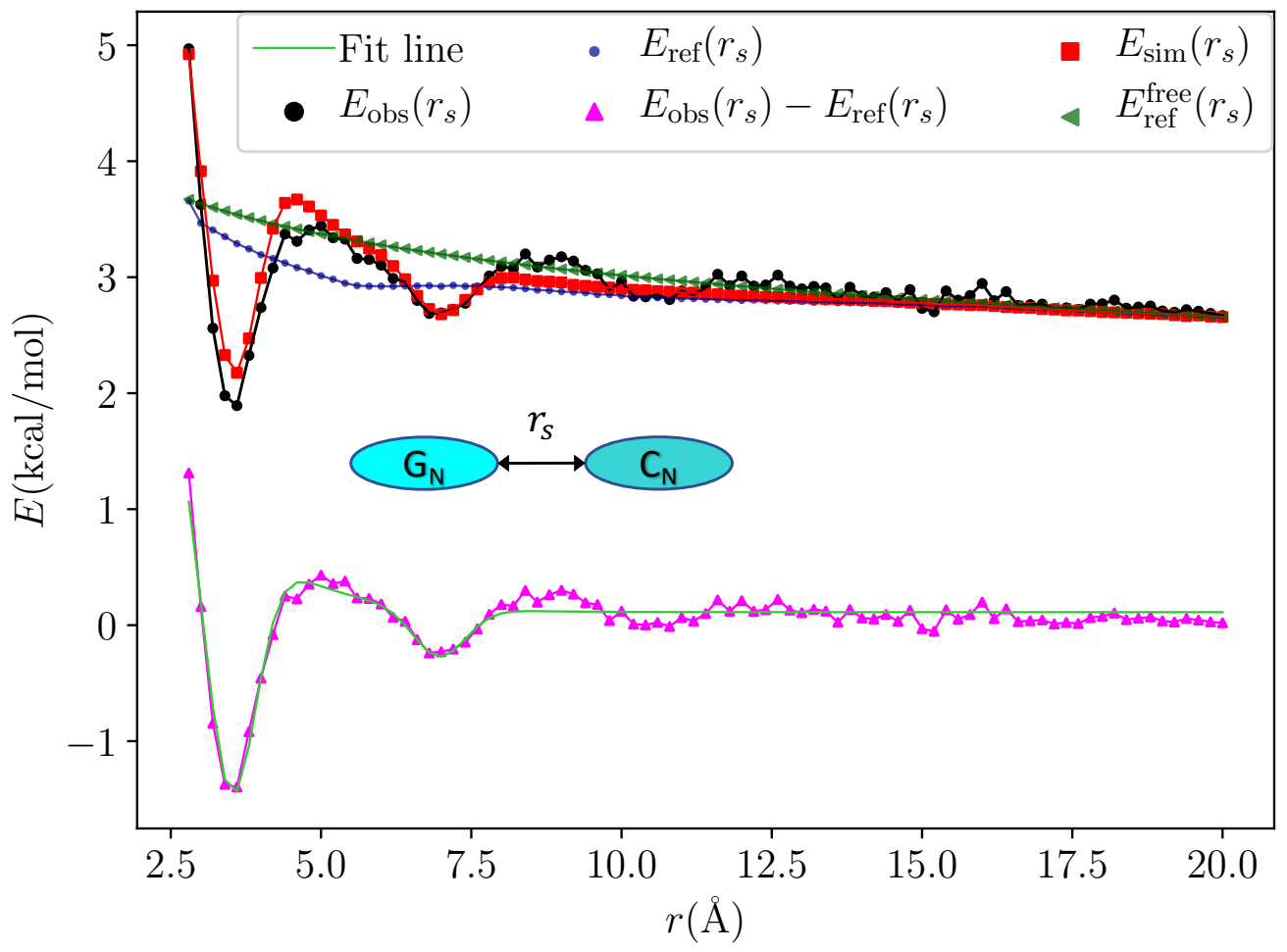

Figure 5.3: Incorporating the first non-local pairwise folding coordinate. After all the required local folding coordinates have been included in $E_{\text {ref }}\left(r_{s}\right)$, non-local base pairing effects can be accounted for. Once this collective variable is included, the energy function produced by simulation $E_{\text {sim }}\left(r_{s}\right)$ matches the observed distribution in the structure database $E_{\mathrm{obs}}\left(r_{s}\right)$. 
Again using statistical mechanics, we show here that the model provides a sound method for parameterizing a predictive force field. After running the simulation with reference energy parameters for backbone bond length and volume exclusion to provide a starting reference state with $E=0$, we obtain the simulated distribution for the first collective variable to be added, $\left.p_{\text {sim }}\left(x_{0}\right)\right|_{0}$. We can write the energy function function from the observed structures as

$$
\left.E\left(x_{0}\right)\right|_{x_{0}}=-\ln \frac{p\left(x_{0}\right)}{\left.p_{\text {sim }}\left(x_{0}\right)\right|_{0}}
$$

where $p\left(x_{0}\right)$ is observed from solved structures. Again, we use $k_{B} T=1$ for convenience. This energy function provides constraints for the next simulation step and is analytically sufficient to reproduce the $p\left(x_{0}\right)$ distribution via simulation.

$$
\left.p_{\text {sim }}\left(x_{0}\right)\right|_{E_{0}}=\frac{\Omega e^{-E\left(x_{0}\right)}}{\sum_{x_{0}} \Omega e^{-E\left(x_{0}\right)}}=p\left(x_{0}\right)
$$

which is the result we obtained in Eq. 5.6. Here, we used the identity $g\left(x_{0}\right)=\left.\Omega \cdot p_{\text {sim }}\left(x_{0}\right)\right|_{0}$, where $\Omega$ is the total number of conformations generated by the simulation, and $g\left(x_{0}\right)$ is the degenerate distribution function obtained from simulation.

Generalizing the estimated joint distribution after consecutive inclusion of $j$ marginal distributions, we find

$$
P\left(x_{j}\right)=P\left(x_{n}\right) \frac{p\left(x_{j}\right)}{\left.p_{\text {sim }}\left(x_{j}\right)\right|_{E_{n}}} \prod_{i=0}^{n} \frac{p\left(x_{i}\right)}{\left.p_{\text {sim }}\left(x_{i}\right)\right|_{E_{n}}}
$$

where $j=n+1, P\left(x_{j}\right)=p_{\text {est }}\left(x_{0}, \ldots, x_{j}\right)$ are the joint distributions estimated from inclusion of $j=n+1$ marginals, and $E_{n}=E_{\text {est }}\left(x_{0}, \ldots, x_{n}\right)$ is the estimated total energy function that provides constraints in the simulation step. The question now is whether we can analytically show that this estimation of the joint probability will provide appropriate 
energy constraints for simulated reproduction of the marginal probabilities observed in the database. To evaluate this, we consider the case where $j=1$ (i.e. the inclusion of the second observed distribution into the energy function). Generating a simulated distribution of $x_{1}$ on the basis of constraints provided by Eq. 5.7, $\left.p_{\text {sim }}\left(x_{1}\right)\right|_{E_{0}}$, we include the observed marginal distribution $p\left(x_{1}\right)$ using

$$
P\left(x_{1}\right)=P\left(x_{0}\right) \frac{p\left(x_{1}\right)}{\left.p_{\text {sim }}\left(x_{1}\right)\right|_{E_{0}}} \frac{p\left(x_{0}\right)}{\left.p_{\text {sim }}\left(x_{0}\right)\right|_{E_{0}}}
$$

where ideally

$$
\frac{p\left(x_{0}\right)}{\left.p_{\operatorname{sim}}\left(x_{0}\right)\right|_{E_{0}}}=1
$$

everywhere, according to Eqs. 5.6 and 5.8. However, retaining this in our expression allows us to correct any small numerical errors that would grow with propagation as we build our estimated joint distribution, maintaining compatibility with the previously incorporated marginal distributions at each step. This can also be viewed as including effects from cross conditional probabilities into the estimated joint distribution. At this stage, our joint energy function has the form

$$
\begin{aligned}
E\left(x_{0}, x_{1}\right) & =-\ln P\left(x_{0}\right) \frac{p\left(x_{1}\right)}{\left.p_{\text {sim }}\left(x_{1}\right)\right|_{E_{0}}} \frac{p\left(x_{0}\right)}{\left.p_{\text {sim }}\left(x_{0}\right)\right|_{E_{0}}} \\
& =\left.E\left(x_{0}\right)\right|_{x_{0}}+\left.E\left(x_{0}\right)\right|_{x_{0}, x_{1}}+\left.E\left(x_{1}\right)\right|_{x_{0}, x_{1}}
\end{aligned}
$$

where as we alluded to earlier, the cross-conditional correction to the energy $\left.E\left(x_{0}\right)\right|_{x_{0}, x_{1}} \approx$ 0 everywhere, which implies that in the next step $\left.p_{\text {sim }}\left(x_{0}\right)\right|_{E_{1}}=p\left(x_{0}\right)$. Since we have explicitly shown our decomposition of the joint into quasi-marginals that account for cross conditionals, the argument we made in Eqs. 5.6 and 5.8 holds, and it can be shown that the simulated distribution of $x_{0}$ remains true to the observed marginal distribution from the 
structure database(see Figs. 5.3 and 5.4 for examples).
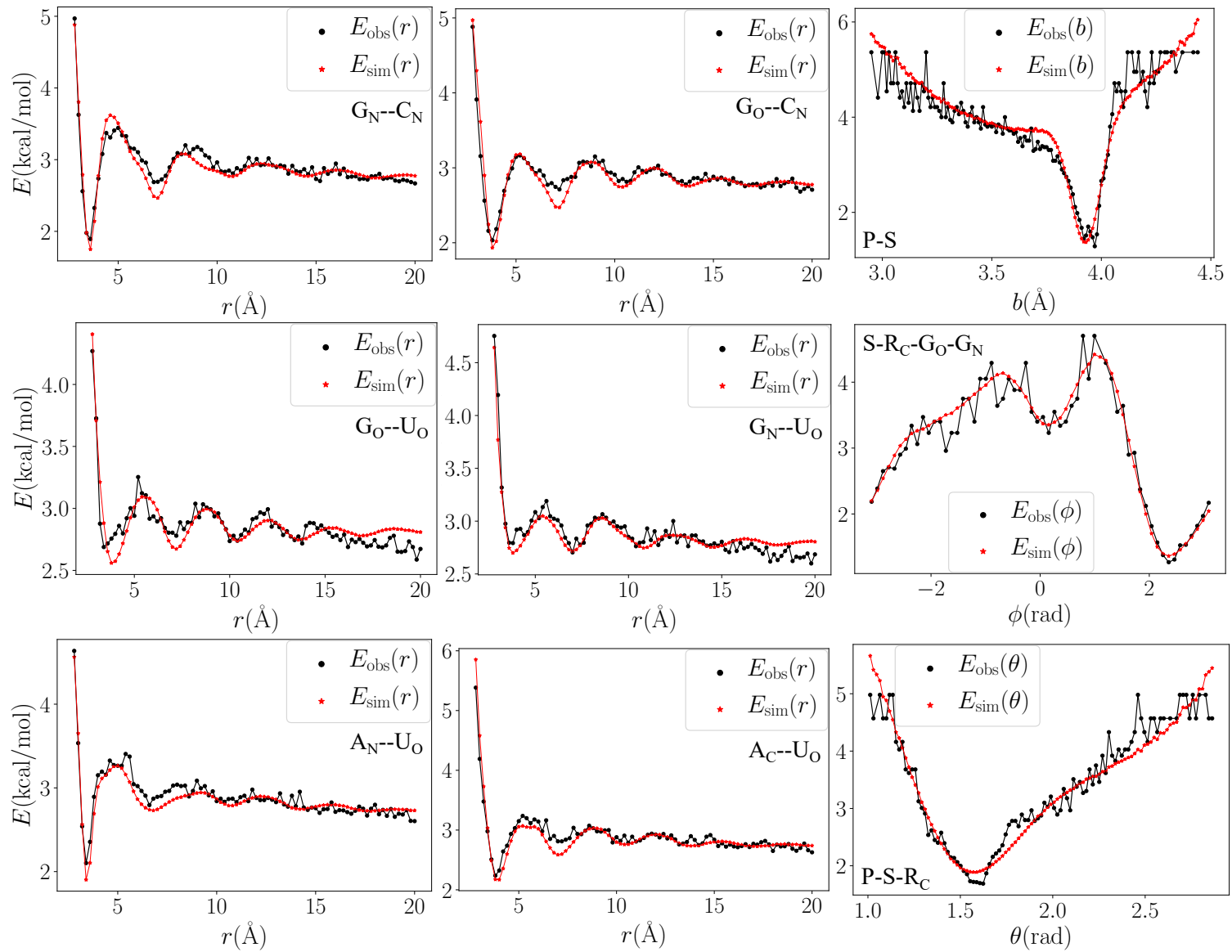

Figure 5.4: A selection of extracted CG energy functions using the IsRNA method. The left and middle columns show non-local energy functions between nucleotides. The right column shows the energy function for a local bond length $b$, torsion angle $\phi$, and bond angle $\theta$.

\subsubsection{Maximizing entropy to optimize model construction}

Although the consecutive procedure detailed above will lead to an estimated joint distribution of our collective variables that is consistent with all of the extracted marginal distributions-regardless of the order that the collective variables are included - the convergence and dimensionality of the energy function can be optimized on the set of all 
considered collective variables using the principle of maximum entropy, capturing all of the information with the fewest possible variables. In order to speed up convergence of the energy function, the IsRNA model incorporates the collective variable that requires the most change to the reference energy function in order to reproduce the observed distribution of the variable via simulation. This well-defined approach leads to an intuitively reasonable order of collective variable inclusion. First, we extract bond length and volume exclusion terms. After that, we include increasingly non-local terms, starting with bond angles, moving to torsion angles, and ending with pairwise distances. Viewing our problem from a Bayesian perspective, our objective is to optimally update from a prior distribution $Q=\left.q_{\text {est }}\left(x_{j}\right)\right|_{E_{n}}$ to a posterior distribution $P=\left.P_{\text {est }}\left(x_{j}\right)\right|_{E_{j}}$, given that the posterior lies within a certain family of possible distributions, $p=\left.p_{\text {est }}\left(x_{0}, \ldots, x_{j}\right)\right|_{E_{j}}$. In our case, the posterior depends on which collective variable we choose to include next $\left(x_{j}\right)$, and we want to include the collective variable that maximizes our information gain at each step. The selected posterior $P$ is that which maximizes the entropy $S[p, q]$. Since prior information is valuable, the functional $S[p, q]$ is chosen so that beliefs are minimally updated to the extent required by the new information.

The relative entropy between a prior distribution $q(x)$ and a posterior distribution $p(x)$ is given by

$$
S[p, q]=-\int p(x) \log \frac{p(x)}{q(x)} d x
$$

This is a measure of surprise that is also called the Kullback-Leibler divergence or the information gain. For our purposes, the term "information gain" helps us intuitively understand how we can use this concept to parameterize a CG force field. Including this concept in our iterative, consecutive procedure will allow us to reach a convergent energy function with the fewest marginal distributions included in our calculation, which has three main bene- 
fits. First, using fewer collective variables to parameterize the force field will accelerate simulations. Second, parameterizing the force field in this fashion requires less computational resources. Third, the concept of maximum entropy is well established, and the direct connection between IsRNA and the theory provides support for the validity of IsRNA. In essence, we check after each simulation to see which candidate observed marginal distribution disagrees the most with the simulated marginal distribution that was constrained by the energy function comprised of all of the previously incorporated marginal and cross conditional distributions. The one that disagrees the most gets incorporated into the joint distribution, providing the minimum information that the model must include to gain compatibility with all of the considered marginal distributions.

As can be seen in Eq. 5.12, our energy constraints supplied by new information at step $j$ can be written as

$$
E_{j}=-\ln \frac{p\left(x_{j}\right)}{\left.p_{\operatorname{sim}}\left(x_{j}\right)\right|_{E_{n}}}
$$

where $j=n+1$. Identifying the candidate $p\left(x_{j}\right)$ as $p$ and the previously known information about the distribution of $x_{j},\left.p_{\operatorname{sim}}\left(x_{j}\right)\right|_{E_{n}}$, as $q$, we see that the principle of maximum entropy agrees with the incorporation of $x_{j}$ into our energy function as long as no other $x_{j}$ can be found that increases the amount of information we can gain in this step.

More directly, we can take the ratio of the estimated joint probability before and after incorporating the new information to see how this is true. Using Eq. 5.9

$$
\frac{p}{q}=\frac{P\left(x_{j}\right)}{P\left(x_{n}\right)}=\frac{P\left(x_{n}\right) \frac{p\left(x_{j}\right)}{\left.p_{\text {sim }}\left(x_{j}\right)\right|_{E_{n}}} \prod_{i=0}^{n} \frac{p\left(x_{i}\right)}{\left.p_{\text {sim }}\left(x_{i}\right)\right|_{E_{n}}}}{P\left(x_{n}\right)}
$$

the joint probability from the previous step cancels in the ratio and leaves us with our proposed information gain times the product of cross conditional probability corrections, 
which we can take for the moment to be approximately unity $(i$. e. not contributing much to the information gain, according to Eqs. 5.6 and 5.8). We therefore find

$$
\frac{p}{q}=\frac{p\left(x_{j}\right)}{\left.p_{\text {sim }}\left(x_{j}\right)\right|_{E_{n}}}
$$

which is the main ratio contributing to the maximum entropy (information gain) formula.

Although we may choose any order of collective variables to include in our energy function (see Figs. 5.2C-D), this method provides us with a method to minimize the number of variables required to accurately simulate structures in agreement with the solved structure ensemble. The principle of maximum entropy method also optimizes the parameterization process-removing the need to guess and check the parameterization order-and supports our choice of marginal energy function. As we can see above, the principle of maximum entropy is consistent with our statistical mechanics approach to update the estimated joint probability distribution and corresponding energy function.

\subsubsection{Applying the model to fold RNA}

Finally, the derived CG force field was applied to study RNA folding behaviors and 3D structure predictions. From coiled states, several RNA molecules were de novo folded by applying the derived IsRNA force field through simulated annealing Molecular Dynamics (MD) in the modified LAMMPS [135] package. In detail, annealing MD simulations were carried out on the structures, scaling the temperature from $350 \mathrm{~K}$ to $200 \mathrm{~K}$ over $500 \mathrm{~ns}$ of simulation time with an integration time step of $\Delta t=1 \mathrm{fs}$. Then, an additional $500 \mathrm{~ns}$ of simulation ( $1 \mu \mathrm{s}$ simulation time in total for each RNA molecule) was performed at constant temperature $(200 \mathrm{~K})$ to sufficiently sample the conformational space and snapshots 
were recorded every $100 \mathrm{ps}$ from the trajectories. To obtain the predicted 3D structure, structures in the $10 \%$ lowest potential energy within the collected snapshots were clustered and the centroid structure of the largest cluster was chosen. As shown in Fig. 5.5, for the 19-nucleotide duplex with a cytosine bulge [156] (PDB ID: 1DQF) and the 12-nucleotide CUUG hairpin loop [157] (PDB ID: 1RNG), the 3D structures predicted by the IsRNA model have heavy-atom root-mean-square deviations (RMSDs) of $2.2 \AA$ and $2.5 \AA$, respectively. Additionally, the folding behaviors of these RNA molecules are characterized by funnel-like plots of the RMSDs with potential energies. Furthermore, simulation in the proposed IsRNA model successfully elucidated the folding pathway for an RNA pseudoknot by predicting a series of kinetically important intermediates and incorporating information from a nanopore experiment and master equation analysis [158]. Taken together, those results demonstrate that the derived CG force field in the IsRNA model could serve as a potent tool to study RNA folding behaviors and to predict RNA 3D structures.

\subsection{Conclusion}

In this work, we simplify the basic theory underpinning the IsRNA model of parameterizing a CGMD force field. Further, we expand the model-explaining the applicability of the model to a general polymer-and discuss our practical approach to attenuate numerical instabilities and errors that may propagate without proper treatment in the model. Although the method of building the force field by consecutively including marginal distributions of individual distance and angle folding coordinates, one-by-one, works for any order of folding coordinate inclusion, we found that optimizing the parameterization process using the principle of maximum entropy allows us to capture all of the dynamic folding information 

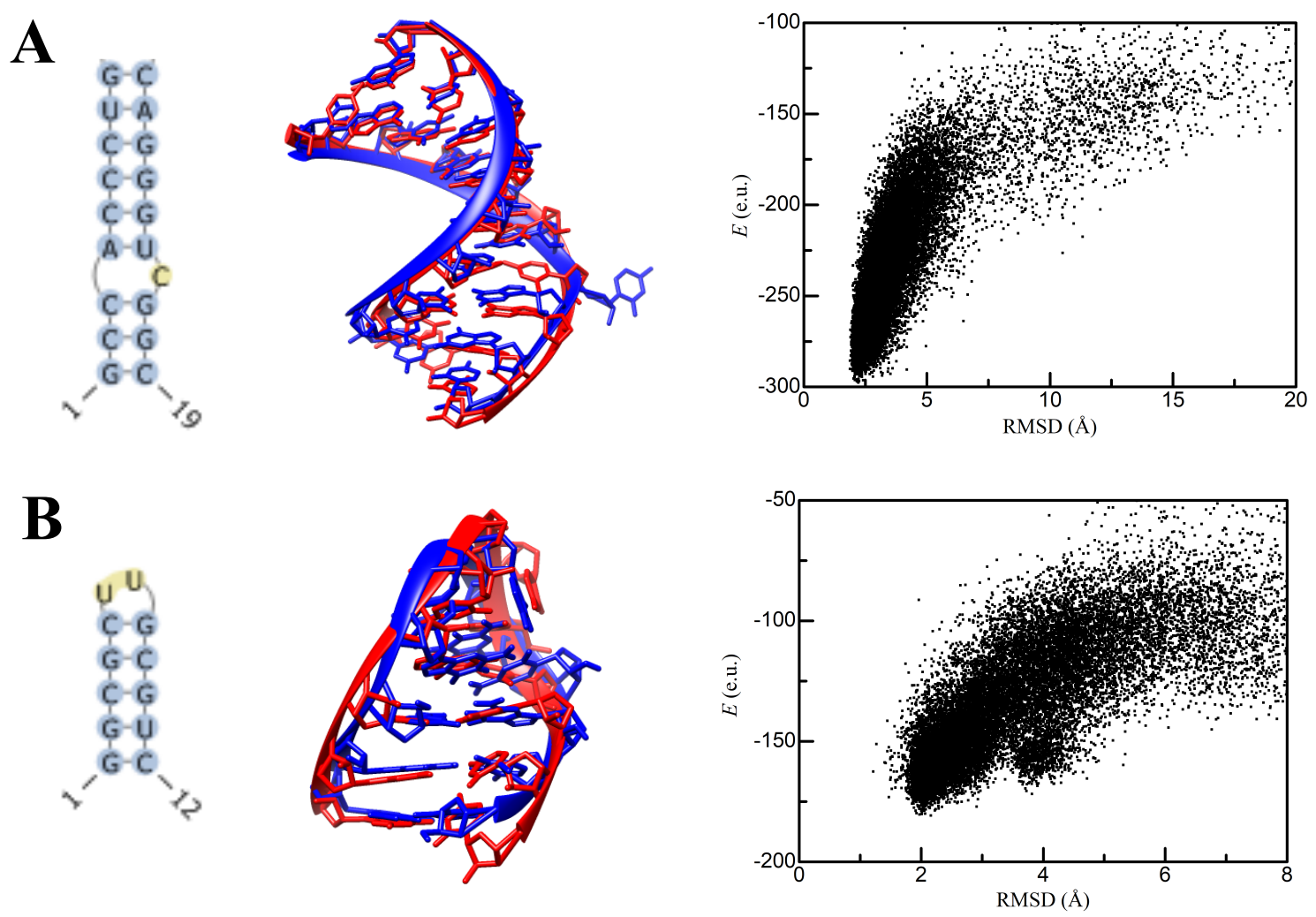

Figure 5.5: Application of the IsRNA model in RNA folding simulations and 3D structure predictions for (A) the helix II of Xenopus laevis 5S rRNA with a cytosine bulge (PDB ID: 1DQF) and (B) the CUUG hairpin loop (PDB ID:1RNG). From left to right: the secondary structure extracted from the native structure, the predicted 3D structure (red color) superposed to the native structure (blue color), and the plot of RMSDs with potential energies in the folding simulation. 
contained in the solved structure database with the fewest possible collective variables. Using the principle of maximum entropy to guide the parameterization process has two main benefits. First, parameterizing the model requires fewer steps, rapidly converging to reproduce observed distributions of our collective variables, and we don't have to guess and check to minimize the number of collective variables required to capture all the information contained in the considered coordinate distributions. Second, the force field uses the fewest possible collective variables selected from the set of considered collective variables, which will make simulations performed with the optimized IsRNA model more efficient. We also provide examples, applying the IsRNA model to fold RNA structures of interest with high accuracy. While we have used this method to successfully model 3D RNA folding, the methodology used in the IsRNA model applies to a wide variety of biopolymer folding problems, and we hope the present study will make the IsRNA procedure more approachable to those desiring to build their own CG force field. 


\section{Chapter 6}

\section{Deciphering nucleotide modification-induced structure and stability changes}

This chapter was published ${ }^{1}$.

Summary: Nucleotide modification in RNA controls a bevy of biological processes, including RNA degradation, gene expression, and gene editing. In turn, misregulation of modified nucleotides is associated with a host of chronic diseases and disorders. However, the molecular mechanisms driving these processes remain poorly understood. To partially address this knowledge gap, we used alchemical and temperature replica exchange molecular dynamics (TREMD) simulations on an RNA duplex and an analogous hairpin to probe the structural effects of modified and/or mutant nucleotides. The simulations successfully predict the modification/mutation-induced relative free energy change for complementary

\footnotetext{
${ }^{1}$ Travis Hurst and Shi-Jie Chen. "Deciphering nucleotide modification-induced structure and stability changes". In: RNA Biol. (2021), pp. 1-11. ISSN: 1547-6286. DOI: $10.1080 / 15476286.2021$. 1882179. URL: https://doi.org/10.1080/15476286.2021.1882179.
} 
duplex formation, and structural analyses highlight mechanisms driving stability changes. Furthermore, TREMD simulations for a hairpin-forming RNA with and without modification provide reliable estimations of the energy landscape. Illuminating the impact of methylated and/or mutated nucleotides on the structure-function relationship and the folding energy landscape, the simulations provide insights into modification-induced alterations to the folding mechanics of the hairpin. The results here may be biologically significant as hairpins are widespread structure motifs that play critical roles in gene expression and regulation. Specifically, the tetraloop of the probed hairpin is phylogenetically abundant, and the stem mirrors a miRNA seed region whose modification has been implicated in epilepsy pathogenesis.

\subsection{Introduction}

Motivated by recent discoveries regarding the fundamental role of ribonucleic acid (RNA) molecules in myriad essential functions within the cell and equipped with new experimental detection techniques, discovery of unique RNA sequences and nucleotide modifications has impressively accelerated [55]. While the existence of modified nucleotides and the need for nucleotide modification to ensure stability of functional RNA structure has been long known [56, 57], the recent improvement of sequencing and detection technologies in the field of epitranscriptomics - natural, post-transcriptional modifications to RNA-has sparked discoveries relating dynamic modification in both coding and non-coding RNAs to diverse modulation of RNA structure, cellular function, and gene expression [55, 58, 59]. Although understanding the effect of nucleotide modification on RNA structure and function is relevant to advancing treatment of common medical conditions-including cancer, 
neurological disorders, diabetes, mitochondrial diseases [60], and HIV [61]—experimental determination of RNA 3D structures remains labor-intensive and difficult due to the dynamical nature of RNA molecules, the negatively charged phosphate backbone, and the difficulty of finding crystal contacts $[62,63]$. Because of new sequencing techniques, the discovery of unique RNA sequences has rapidly outpaced experimental determination of corresponding 3D structures. To bridge the gap between sequencing data and structural information, we need accurate computational structure prediction tools, which are important and necessary for advancing RNA 3D structure determination. Including the impact of nucleotide modification in calculations of RNA structure and stability is crucial to accurately compute and predict the structure, energetics, and kinetics.

Even simple modifications, such as methylation of a nucleotide, can have profound impacts on human health. For example, $\mathrm{N}^{6}$-methyladenosine $\left(\mathrm{m}^{6} \mathrm{~A}\right)$ is often found in the 3'UTR and is known to regulate RNA localization and splicing [159]. By regulating effects of the methyl-CpG binding protein 2 (MeCP2), suppression of $\mathrm{m}^{6} \mathrm{~A}$ modification may alleviate disease symptoms in fragile X-linked mental retardation; conversely, excessive suppression of $\mathrm{m}^{6} \mathrm{~A}$ is associated with autism $[160,161]$. In epilepsy-associated miRNA, $\mathrm{m}^{6} \mathrm{~A}$ modification of the seed region could affect miRNA maturation and binding to mRNA targets, which may impact the severity or presence of epilepsy $[162,163]$. Endometrial cancer, hepatic cancer, breast cancer, and leukemia tumorigenicity have been linked to upregulation or suppression of $\mathrm{m}^{6} \mathrm{~A}$, making $\mathrm{m}^{6} \mathrm{~A}$ and its regulators targets for cancer detection and therapy [164-168]. Similarly, ribothymidine $\left(\mathrm{m}^{5} \mathrm{U}\right)$ is elevated in breast cancer cells, and the tRNA methyltransferase 11 homolog (TRMT11) responsible for methylating guanosine to $\mathrm{N}^{2}$-methylguanosine $\left(\mathrm{m}^{2} \mathrm{G}\right)$ is associated with prostate cancer $[169,170]$. However, the underlying molecular mechanisms from which these associations arise remain poorly 
understood.

The growing set of known, natural modifications add to the complexity of accurate structure prediction. While determining empirical nearest neighbor energy parameters for the canonical nucleotides provided secondary structure insights and computational prediction parameters [70], performing this task for all the permutations of the more than 170 known modified nucleotides would be difficult and time consuming. A limited number of models support the incorporation of energetic parameters for modified nucleotides [71, 72], but no current predictive model completely accounts for the impact of these modifications on RNA structure [73]. Although studies have been carried out to computationally model the geometry and base pairing character of the modified RNA nucleotides [74, 75] and an all-atom force field has been developed for 107 of the known modifications [76], few predictive parameters that are validated by experimental data have been published. Information about modifications may be implicitly included in some prediction algorithms, where knowledge of structural motifs drives the energy function. However, the implicit inclusion of these modifications hampers the models in predicting the truly unmodified RNA structure because the assumption of modification is intrinsic to the models. To increase accuracy and usefulness of RNA structure prediction, we should explicitly include these naturally occurring modifications in the models.

In light of the rapid development and increasing importance of the field of epitranscriptomics, this work seeks to find relationships between nucleotide modification and functional RNA structure. RNA molecules serve to regulate a variety of cellular functions, and the functionality of an RNA is determined by its structure. While previous computational work has mainly focused on computationally determining thermodynamic parameters for unmodified duplexes with some mismatched base pairs [171, 172], some experimental stud- 
ies have extracted thermodynamic parameters for some interactions involving modified nucleotides [173-177], and recent work used a combined molecular dynamics/quantum mechanics approach to successfully reproduce experimental free energy changes for base pairs with modified nucleotides [178], indicating sufficient experimental progress has been made to validate computational studies of modified RNA thermodynamics. To gain insight into RNA structure and function, many experimental and computational studies focus on hairpins. Hairpin loops are a ubiquitous structural motif in RNA, have been implicated as initiators of RNA folding, and commonly engage in tertiary interactions in protein-RNA interfaces and RNA recognition sites [179-181].

Here, we performed alchemical simulations on an RNA duplex and its modified and/or mutated derivatives to calculate relative free energy changes and directly compare with thermal denaturation experiments [175]. Furthermore, we use structural information from alchemical simulations to support mechanisms driving modification and mutation-dependent stability changes. In the alchemical approach, a system is changed from one state of interest to another using convenient, unphysical ("alchemical") intermediates in order to estimate the free energy difference between the states. For instance, a nucleotide base in an RNA can be changed from the wild-type base into a modified or mutated base through a series of steps that cause wild-type base atoms to disappear and modified/mutated base atoms to appear, providing a convenient path between chemically different states. Alchemical simulations are a widely used technique for estimating free energy changes in applications ranging from drug design to structure stability studies [182-185]. The optimal methodology for carrying out these simulations is a topic of vigorous, current research $[186,187]$. Due to the pervasiveness and importance of hairpins, we used temperature replica exchange molecular dynamics (TREMD) —also called parallel tempering — to explore the effect of methylation 
and/or mutation on the potential of mean force (PMF) for an analogous RNA hairpin and discern how these modifications affect the propensity for the RNA to form a functional hairpin. While thermodynamic experiments are vital facilitators for the understanding of RNA structure and dynamics, viewing the RNA on an atomistic level using TREMD yields structural information that is absent in thermal denaturation experiments. TREMD is an enhanced sampling method with several advantages [184, 188], and several studies on hairpins and tetraloops have used TREMD to explore RNA folding dynamics [189-201]. In comparison to other popular methods for estimating PMFs—such as umbrella sampling, steered molecular dynamics, or adaptively biased molecular dynamics-TREMD is an equilibrium method that does not require a priori knowledge or assumptions about which collective variables best identify the relevant states of the system [188]. After running the TREMD simulations, we examine the PMF projected along any collective variable(s) we choose, and the PMFs are not biased from sampling being driven along any specific coordinate. Some disadvantages of TREMD are that we cannot guarantee complete sampling on a short timescale, and TREMD is more computationally demanding than non-equilibrium or collective variable-driven methods for determining PMFs. However, over long enough timescales with small enough systems, PMFs generated by TREMD are reproducible, and we can learn about the folding of the system without pre-existing coordinate biases. Both the hairpin and duplex contain a complementary 5'-GAC-3' helical region that mirrors the seed region of miRNA-134, and the adenosine in this miRNA is a potential methylation target that has been implied to be foundational to epilepsy pathogenesis [162]. The hairpin also contains the most common tetraloop (UUCG), which forms a nucleation site for RNA folding [202]. The rugged free energy landscape of this hairpin provides a wealth of information on how modifications in the loop or the stem can constrain or broaden the structural 
possibilities. Comparison of the PMF with thermal denaturation data shows agreement, which validates our approach. Understanding structural and dynamical changes driven by these nucleotide modifications may help discern molecular disease mechanisms and rational design of RNA nanotherapeutics [68, 203].

\subsection{Materials and Methods}

\subsubsection{Selecting and building systems}

The complementary 5'-UACGACUG-3' RNA duplex (see Fig. 6.1A) probed in thermal denaturation experiments was built using the Amber 18 nucleic acid builder module [134, 204]. Experimental data is reproduced in Table C.1. Single-stranded reference states were formed by deleting the unchanged strand in the duplex. The single-stranded reference state allows us to remove free energy changes that do not directly affect the free energy of duplex annealing. Hybrid structures were manually constructed from the duplex and single stranded PDB structures. Identified as a potential site for $\mathrm{m}^{6} \mathrm{~A}$ methylation, the seed region of miRNA-134 contains an analogous 5'-GAC-3' sequence, and modification of A to $\mathrm{m}^{6} \mathrm{~A}$ may affect maturation of the miRNA and its ability to bind with proteins or target mRNA [162]. Upregulation of this miRNA has been associated with epilepsy [163].

An unmodified, folded, wild type hairpin (WT) was taken from an enzyme-activating fragment of human telomerase RNA (PDBID: 1Z31). The 10 nucleotide RNA hairpina tetraloop with a three base pair stem-has the sequence 5'-gacUUCGguc-3', so it also contains the complementary 5'-GAC-3' stem probed in the duplex experiments [175] and suspected to play a role in epilepsy pathogenesis via A to $\mathrm{m}^{6} \mathrm{~A}$ modification [162] (see 

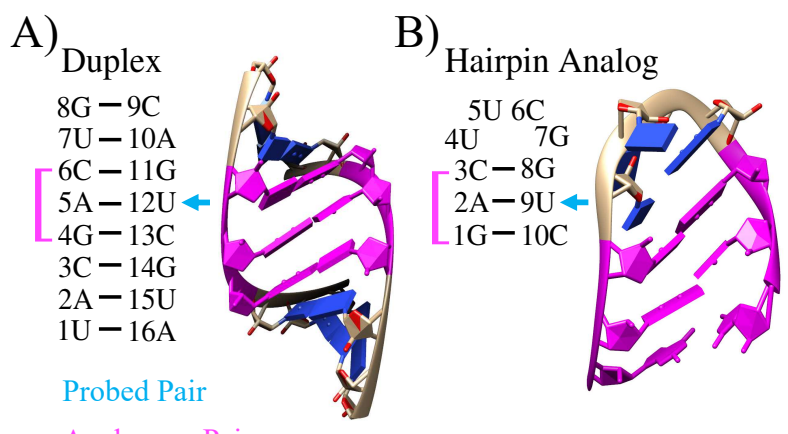

Analogous Pairs

Figure 6.1: Unmodified 2D and 3D structures. For the A) duplex and B) hairpin, analogous regions are highlighted in magenta and the experimentally probed base pair is denoted with a blue arrow. Nucleotides are numbered from 5' to 3' ends.

Fig. 6.1). Further, this hairpin analog of the duplex was selected because previous studies have determined that the UUCG tetraloop with a CG closing base pair exhibits two-state behavior with high stability, is phylogenetically abundant, and has been implicated as a nucleation site during RNA folding [202].

Mutations on the hairpin and duplex were performed using UCSF Chimera [205]. In accordance with modrna08 [76] standards, methylation was accomplished by renaming the modified residue in the PDB file, and the geometrically correct, modified residue was subsequently included in the parameter/topology and input coordinate files built by the tleap module in Amber 18 [134]. Starting from a folded 3D structure, all simulations were conducted with the recommended OL3 force field for RNA molecules and the modrna08 [76] force field for modified nucleotides.

Alchemical and TREMD simulations were performed on the duplexes and hairpins using standard methods described in detail in SM Sections C.2 and C.3, respectively. For alchemical production runs, $112.4 \mathrm{~ns}$ simulations-corresponding to alchemical control parameter values $(\lambda=0.0,0.1, \ldots, 1.0)$-were used for each of the three legs (Discharge, Lennard-Jones, Charge) in the thermodynamic cycle (see Fig. 6.2) for both the duplexes of 
interest and the single-stranded reference states. Doubling the simulation time did not improve the agreement with experiment or reduce the calculated uncertainty (see Table C.2), which aligns with previous findings, where longer simulations do not necessarily improve results due to bias from the initial velocities [206]. For hairpin TREMD production runs, 24 replicas were simulated, each for $300 \mathrm{ns,}$, with temperatures ranging from 265 to $365 \mathrm{~K}$, as suggested by the predicted melting curve given by VfoldThermal [90] (see Fig. C.1).

\subsubsection{Using alchemical simulation results to estimate free energy changes}

To quantify the effect of nucleotide modification on RNA energetics, we performed alchemical simulations on the RNA duplex found in experiments and its modified/mutated derivatives. In thermodynamic integration (TI) calculations, the system transitions from one end state of interest to another through unphysical (alchemical) states according to a mixed potential $U(\lambda)$ in order to numerically estimate free energy change from the transformation between states, where the alchemical control parameter $\lambda$ allows us to move from the starting state to the end state using a mixed potential [207]. This change from one state to another is an alchemical perturbation that is accomplished via alchemical simulations of the system. In order to form a thermodynamic cycle, we use the single stranded state as a reference state (see Appendix C, Section C.4 for more details). The relative free energy due to modification $\Delta \Delta G$ is determined using

$$
\Delta \Delta G=\Delta G_{\mathrm{A}}-\Delta G_{\mathrm{B}}=\Delta G_{\mathrm{C}}-\Delta G_{\mathrm{D}}
$$


Practically, we break the cycle into smaller steps, where for example,

$$
\Delta G_{\mathrm{A}}=\Delta G_{1}+\Delta G_{2}+\Delta G_{3}
$$

as described in Fig. 6.2.

To analyze the data and validate the completeness of our sampling, the first-order polynomial (trapezoidal) integration scheme for TI (TI-Trapezoid), the cubic spline integration scheme for TI (TI-Cubic), the Bennett acceptance ratio (BAR), and MBAR energy estimators were used (see Appendix C, Section C.7 for discussion and Fig. C.2 for comparisons). While many estimators are available, TI and MBAR provide the most accurate results with the smallest amount of data within their distinct realms, and inconsistency between the two can indicate poor phase-space overlap or insufficient sampling [208]. Estimator uncertainties were propagated by summing uncertainties of each leg of the thermodynamic cycle in quadrature (see Appendix C, Section C.8). Results provided here are based on data from eight repeat runs because standard deviations provide more accurate assessments of uncertainty than the estimators and because averaged results remove bias due to initial conditions. Using alchemical analysis software [209], uncorrelated, independent samples were automatically extracted from the simulation data.

\subsubsection{Analyzing TREMD trajectories}

Structure clustering was performed on the basis of orthogonal reaction coordinates determined from principal component analysis, and representative frames corresponding to the centroid of each cluster were extracted for structural analysis (see Appendix C, Section C.3). Additionally, the RMSD from a common, unmodified reference structure and 


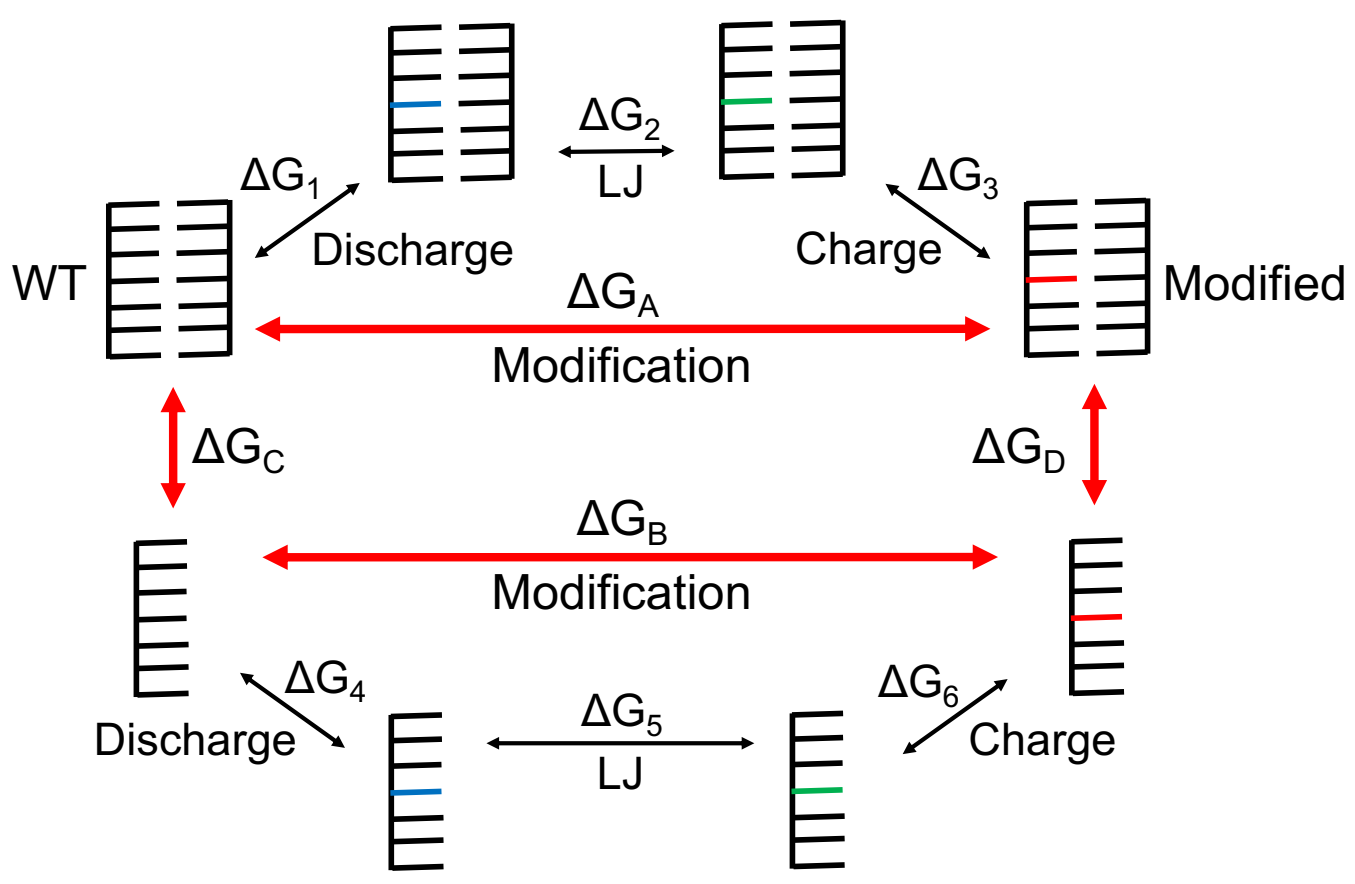

Figure 6.2: A thermodynamic cycle formed by the states indicated by red arrows. In order to separate the electrostatic and Lennard-Jones $(\mathrm{LJ})$ contributions to the free energy change, we use three main steps to go from the WT to the modified states. The first step, labeled "Discharge", removes all charge from the atoms in the starting residue. The blue residues are electrically neutral WT residues. The second step, labeled "LJ", replaces atoms and bonds in the WT residue with atoms and bonds from the modified residue. Because the vanishing and appearing atoms are all electrically neutral in this step, the LJ potential is the contributor to the free energy change. The green residues are electrically neutral, modified residues. The third step, labeled "Charge", reintroduces charge to the modified residue to bring us to the final, modified state. The red residues represent the electrically charged, modified residues. These steps must be done for both the duplex and reference (single stranded) states in order to calculate the relative free energy change due to modification $\Delta \Delta G$. 
the end-to-end distance (E2E) were determined using CPPTRAJ [210] for each snapshot in each run. A Python implementation of the multistate Bennett acceptance ratio (MBAR) [211] estimator was used to determine the potential of mean force (PMF) for a simulation window. The PMF projects a convoluted, multivariate free-energy landscape onto well-defined collective variables (reaction coordinates that ideally describe the dynamics of interesting aspects of the system), supplying quantitative and qualitative information to build our knowledge of the kinetics and energetic preferences of the system. Although full convergence of this system would probably require runs on the order of several $\mu \mathrm{s}$, calculating the PMFs with increasing simulation time confirmed reasonable convergence (see Fig. C.3).

For the purposes of comparing our simulated results to experimental denaturation data, we estimate the free energy of hairpin formation due to modification from the calculated PMFs (see Fig. 6.1 for hairpin structure). Clustering the structures and identifying the representative frames - the frame closest to the centroid of the cluster-and their reaction coordinates allows us to verify the location of the native hairpin $(\mathrm{NH})$ conformation and view long-lived alternative conformations in the energy landscape. See Appendix C, Section C.10 for details.

\subsection{Results and Discussion}

To connect thermal denaturation experiments to theory, we performed alchemical simulations on the experimentally probed, WT duplex and its modified and/or mutated variants, which enabled calculation of relative free energy changes due to denaturation. After establishing the trend between free energies from simulation data and experiment on the 
duplex, we used TREMD simulations of a hairpin analog with a phylogenetically abundant tetraloop to show that the trend holds. In addition to the folding stability from the duplex simulations, the general agreement between the hairpin and duplex calculations with thermal denaturation experiments show the validity of the simulation data, so we evaluated how the hairpin folding mechanism change when perturbed by small modifications. The results of this analysis may be beneficial for understanding mechanisms driving modificationrelated disease and for guiding RNA nanotherapeutic design of hairpin motifs.

\subsubsection{Comparing duplex simulation results to thermal denaturation data}

Results from the alchemical simulations show that we can accurately rank the free energy change of duplex formation due to modification or mutation $\Delta \Delta G$ using alchemical analysis. Because we used independent simulations to modify and mutate the duplex, more errors were propagated in cases where multiple perturbations were applied. To alleviate this issue, we performed calculations for twice-perturbed systems in both alchemical directions (see Fig. C.2 and Section C.8 in SM). The modify-then-mutate direction first imposed the modification of the $5 \mathrm{~A} / 12 \mathrm{U}(\mathrm{A}-\mathrm{U})$ base pair to $\mathrm{m}^{6} \mathrm{~A}-\mathrm{U}$; subsequently, the mutation of $12 \mathrm{U}$ to $\mathrm{C}, \mathrm{G}$, or $\mathrm{A}$ was performed. In the mutate-then-modify direction, the mutation occurs first, followed by the modification. The success of these twice-perturbed results depends on the accuracy of the calculations from the singly-perturbed steps $\left(\mathrm{m}^{6} \mathrm{~A}-\mathrm{U}, \mathrm{A}-\mathrm{C}, \mathrm{A}-\mathrm{G}\right.$, and A-A). Combined results from modify-then-mutate and mutate-then-modify are provided in Fig. 6.3, where the averaged uncertainties for twice-perturbed systems are on par with uncertainties for singly-perturbed systems (see Table C.2 and Appendix C, Section C.8 for details). Provided that the paths of perturbation are exhaustively sampled, our analysis 
indicates that combined results from twice-perturbed systems yield uncertainties that are roughly equivalent to singly-perturbed uncertainties. Given the small amount of data $(<$ 400 uncorrelated data points for each $\lambda$ value), the calculated free energies from alchemical simulations were broadly successful in ranking the experimentally determined $\Delta \Delta G$ values, achieving a 0.93 Spearman rank correlation coefficient between our predicted $\Delta \Delta G$ and the experimental ranking, which shows that the ranking of the calculated free energies trends with experiment. For the unmodified mutant systems, the relative free energies estimated from the alchemical simulations appear to better match the thermal denaturation data than estimates calculated by applying the Turner rules, using the tabulated nearest neighbor Watson-Crick helices and 1x1 internal loop parameters [212]. Some of the differences between the experimental results and Turner parameter estimates or the simulation estimates could be due to a difference between the solution conditions of the experiment. The simulations do not perfectly match the experimental solution conditions of $100 \mathrm{mM}$ $\mathrm{NaCl}, 10 \mathrm{mM} \mathrm{MgCl} 2,10 \mathrm{mM}$ Na-PIPES (pH 7.0), $5 \mathrm{M}$ duplex, and the Turner parameters assume a $1.0 \mathrm{M} \mathrm{NaCl}$ solution.

The overlap matrices produced by alchemical analysis software [209] indicate that sufficient phase-space overlap was accomplished by our $\lambda$ schedule (Fig. C.4). Furthermore, convergence plots indicate that we obtained reasonable convergence because timedependent estimates of the free energy in the forward and reverse sampling directions sufficiently overlap (Fig. C.5). The mutate-then-modify version of the twice-perturbed alchemical simulation produces consistently lower values of $\Delta \Delta G$ (Fig. C.2), indicating that the agreement in magnitude with experimental values is better when the smaller perturbation is applied first, but the ranking is in slightly better agreement with experimental results when both directions are used. The improvement in ranking from using both directions 


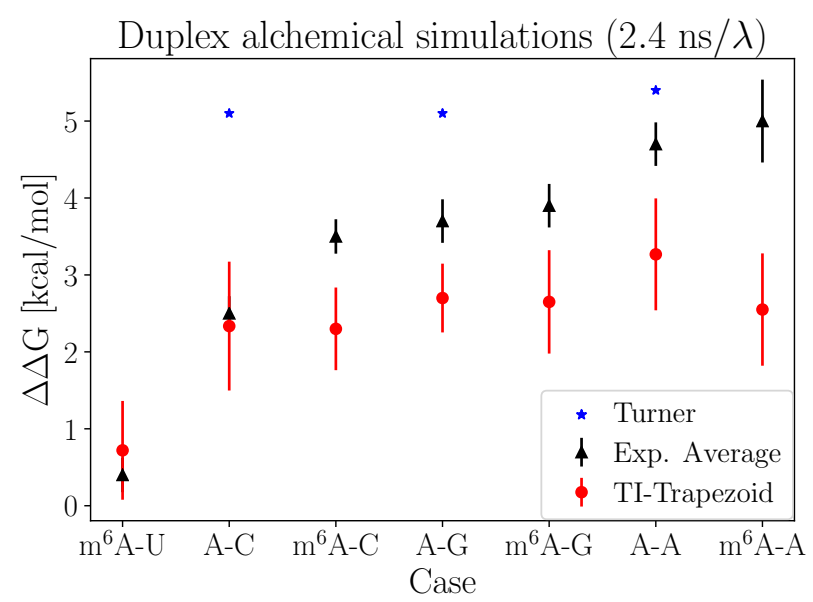

Figure 6.3: Calculated relative free energy changes using TI-Trapezoid. Experimental [175] (calculated) results and error bars are shown in black (red). The results for twice-perturbed cases are the average of the results from the modify-then-mutate and the mutate-then-modify alchemical paths. Estimates from applying the Turner rules are shown in blue [212].

is probably due to using twice as many simulations to obtain the averaged results, while the improved agreement in magnitude with experiment from using only the modify-thenmutate direction is probably due to applying the weaker perturbation first, which prevents the system from drifting too far away from the state of interest during the mutation step of the simulation. Mutating the system first causes the stronger perturbation to be applied for longer, which causes more drift away from the standard duplex structure of interest.

\subsubsection{Determining mutation/modification-induced structural mecha- nisms driving duplex stability changes}

Primarily a tool for free energy estimation, alchemical simulations are not optimized to identify dynamics of structure change because most of the structures are generated under an alchemical potential that is not reflective of reality. However, the structures produced under pure potentials ( $\lambda=0$ or 1 ) may be evaluated to check whether large, invalidating duplex 
deformations occurred during the simulations and to identify new interactions and preferred conformations. We found that there was not a lot of bulging or topological deformation from the mutations and modifications, even for mutations that result in purine-purine base pairs. For instance, the average heavy atom backbone RMSDs for the probed base pair from a reference structure (see Appendix C, Subsection C.2.2 for more details) were 1.3 and 1.6 $\AA$ for WT conformations and mutated and/or modified conformations, respectively. The average distance between phosphate groups of the two residues remained relatively consistent across all cases $(20.0 \pm 0.8 \AA)$, indicating that the small average RMSD change is likely due to small structure changes to accommodate steric strain rather than large bulging of the duplex topology at the probed site (see Appendix C, Subsection C.2.2 for more details).

Specifically, the mutation of $12 \mathrm{U}$ to $12 \mathrm{~A}$ to form the A-A base pair did not result in a large physical bulge in the duplex. Instead, the $12 \mathrm{~A}$ residue rotated to expose its sugar edge to the Watson-Crick edge of 5A. This behavior also occurred in the modify-then-mutate $5 \mathrm{~m}^{6} \mathrm{~A}-12 \mathrm{~A}$ structure. Because no more hydrogen bonds are disallowed in the $5 \mathrm{~m}^{6} \mathrm{~A}-12 \mathrm{~A}$ structure than in the A-A mutated structure, the enhanced stability of this duplex in the alchemical free energy calculation relative to the 5A-12A mutant duplex is likely from the enhanced stacking strength of $5 \mathrm{~m}^{6} \mathrm{~A}$ with the $6 \mathrm{C}$ residue. This mechanism may also explain the increased $5 \mathrm{~m}^{6} \mathrm{~A}-12 \mathrm{C}$ stability in comparison to the $5 \mathrm{~A}-12 \mathrm{C}$ mutation. Structural details and further topology analysis can be found in Fig. C.6. The small structure changes shown in the simulations did not appear to invalidate the results because we did not see any major deformations or structures that greatly deviated from the A-form helix structure, and the general trend of the results with experimental data further supports the validity of the simulations. 
To determine the effect of base pairing on the conformation of the $\mathrm{m}^{6} \mathrm{~A}$ residue, we examined the modified structures in both paired (duplex) and unpaired (single-stranded) states. In the single-stranded state, the methyl group prefers to point toward solution but is free to occasionally rotate into the sterically strained position. In the base-pairing duplex state, the methyl group prefers to be in a sterically strained position to facilitate the hydrogen bonding between the methylamino group and 12U (Fig. C.7). This supports the "spring-loaded" mechanism proposed in previous studies [175] to explain the instability caused by the methylation. The methylation makes natural formation of a base pair less probable. Similarly, once the pair is broken, the methyl group may act to push the pairing partner away and prevent reformation of the hydrogen bond.

\subsubsection{Comparing hairpin PMFs to thermal denaturation data}

To test the robustness of our hairpin TREMD simulation results, we compared the calculated $\Delta \Delta G$ from the 2D RMSD/E2E PMFs to thermal denaturation data [175]. While the experimental system consists of RNA duplexes containing eight base pairs (5'-UACGACUG3'), the stem of our simulated hairpin (5'-gacUUCGguc-3') contains the complementary 5'-GAC-3' sequence probed by these experiments. The hairpin also contains a tetraloop that the experimental duplexes clearly lack, and the simulations were performed with 1 $\mathrm{M} \mathrm{Na}^{+}$counterions and neutralizing amounts of $\mathrm{Cl}^{-}$ions, while the duplex experiments were performed with $100 \mathrm{mM} \mathrm{NaCl}$ and $10 \mathrm{mM} \mathrm{MgCl}_{2}$. Additionally, because the hairpin rapidly unfolds when noncanonical base pairs are introduced, there are few conformations populating the folded region of mutant PMFs after a notable amount of time. To accommodate the dearth of $\mathrm{NH}$ conformations at longer simulation times for noncanonical cases, we used the 5 to $300 \mathrm{~ns}$ window, where the NH state is still present on the PMF for all of the 


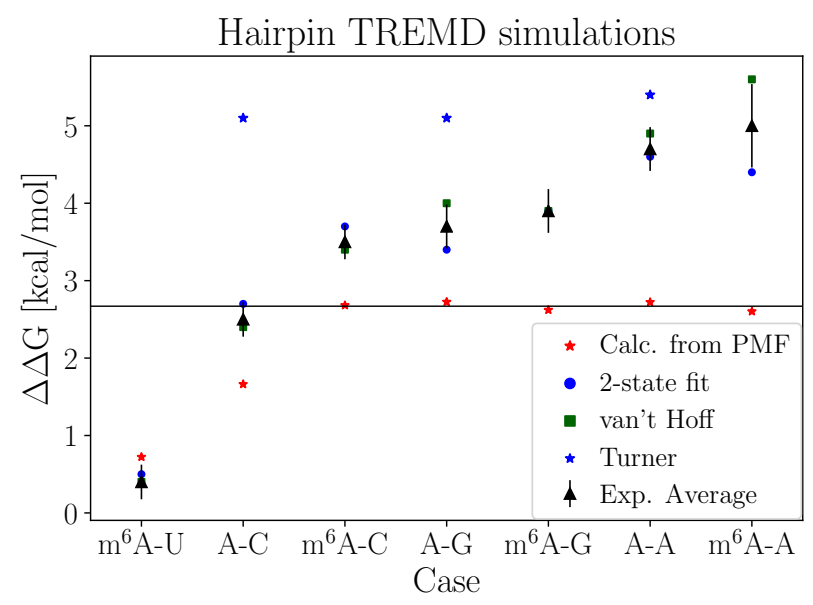

Figure 6.4: Experimental [175] and calculated relative free energy changes due to modification $\Delta \Delta G$. For the eight base pair duplex, experimentally determined free energies calculated from two-state curve fits, van't Hoff linear plots, and averages between the twostate fit and van't Hoff fit are labeled "2-state fit", "van't Hoff", and "Exp. average", respectively. The values for $\Delta \Delta G$ calculated from the RMSD/E2E 2D PMFs of the hairpin analog are labeled "Calc. from PMF". Descriptive identifiers are provided to describe modifications and/or mutations (see Fig. 6.1): for example, " $\mathrm{m}^{6} \mathrm{~A}-\mathrm{U}$ " represents the modification of $2 \mathrm{~A}(5 \mathrm{~A})$ to $\mathrm{m}^{6} \mathrm{~A}$ in the simulated hairpin (experimental duplex), maintaining the canonical base pair with $9 U$ (12U). Likewise, "A-C" represents the hairpin (duplex) mutation of $9 \mathrm{U}(12 \mathrm{U})$ to $\mathrm{C}$. All hairpin (duplex) mutations were imposed on 9U (12U). The gray line indicates the free energy plateau, where the hairpin becomes completely destabilized. Experimental error bars are shown with respect to the experimental average. Estimates from applying the Turner rules are shown in blue. 
cases. Despite the stark differences in the systems and although the systems are not fully equilibrated at $5 \mathrm{~ns}$, we can make a connection from our simulated hairpin to these experimental duplexes through the relative change in free energy of formation due to modification $\Delta \Delta G$.

While the calculated $\Delta \Delta G$ consistently underestimates the experimental value for noncanonical base pairs, the computed free energy for $\mathrm{m}^{6} \mathrm{~A}-\mathrm{U}$ is within experimental error. This indicates that large destabilizations due to noncanonical base pairing interactions have a weightier impact on our short stemmed hairpin than on the corresponding experimental duplex, where the five extra base pairs help stabilize the folded helix conformation (see Fig. 6.4). Beyond a destabilizing threshold, the NH conformation rapidly disappears from the PMF, and $\Delta \Delta G$ plateaus at a maximum, above which we cannot accurately determine the destabilizing effect due to modification from this probe: the three base pair stem of the hairpin becomes totally disrupted and cannot differentiate between a $2.7 \mathrm{kcal} / \mathrm{mol}$ disruption and a $5 \mathrm{kcal} / \mathrm{mol}$ disruption. However, when methylations are applied to the canonical hairpin, $\Delta \Delta G$ remains below the threshold set by the noncanonical cases. Interestingly, this threshold at $\sim 2.7 \mathrm{kcal} / \mathrm{mol}$ is an estimate of the absolute stability of the WT NH state because it is the maximum destabilization the hairpin can withstand: i.e., the WT NH absolute free energy of formation estimated by this hairpin study is $\Delta G^{300 K} \approx-2.7 \mathrm{kcal} / \mathrm{mol}$ (see gray line in Fig. 6.4). This prediction is in agreement with experimental thermal denaturation studies of the UUCG tetraloop, where results from several hairpins-5' -gcgUUCGcgc-3' ( $\left.\Delta G^{310 K}=-2.2 \mathrm{kcal} / \mathrm{mol}\right), 5^{\prime}$ $\operatorname{cgcUUCGgcg-3}{ }^{\prime}\left(\Delta G^{310 K}=-3.65 \pm .41 \mathrm{kcal} / \mathrm{mol}\right), 5^{\prime}$-ggagUUCGcucc-3' $\left(\Delta G^{310 K}=\right.$ $-2.9 \pm .14 \mathrm{kcal} / \mathrm{mol})$, and 5'-ggacUUCGgucc-3' $\left(\Delta G^{310 K}=-5.07 \pm .22 \mathrm{kcal} / \mathrm{mol}\right)-$ showed the enhanced stability provided by the CG closing base pair when adjacent to 
UUCG tetraloops [202].

While the trend calculated from the hairpin PMF somewhat follows the trend from the experimental duplex, the hairpin calculation reaches a plateau and fails to accurately assess free energy changes due to large disruptions. Experimentally, the five extra base pairs in the duplex lock the duplex in place, which facilitates better assessment of larger disruptive energies. In contrast, the three base pair stem of the hairpin becomes totally disrupted when mutations destabilize the hairpin beyond the free energy of formation. However, small perturbations due to methylation are below the threshold, and in the context of these small perturbations to the energy landscape, the analogy between the hairpin and duplex holds.

\subsubsection{Investigating modification-induced hairpin structural alterations}

The results from methylations with the otherwise canonically base pairing hairpin were extracted from 50 to $300 \mathrm{~ns}$ simulation windows for comparison to the unmodified WT. The results provided here are extracted from the 2D PMFs using RMSD and E2E as reaction coordinates (RMSD/E2E PMFs) because the physical meanings of these reaction coordinates are clear. In Fig. 6.5, the PMF for the WT, the $2 \mathrm{~m}^{6} \mathrm{~A}$ modification with thermal denaturation data and heavily implicated in disease, a destabilizing $5 \mathrm{~m}^{5} \mathrm{U}$ modification, and a stabilizing $7 \mathrm{~m}^{2} \mathrm{G}$ modification represent a summary of our findings. Discussions and PMFs for other modifications can be found in Figs. C.8-C.16.

The unmodified WT RMSD/E2E PMF shows a clear preference for a folded, NH conformation with a low RMSD and an E2E distance of about $10.7 \AA$ (see Fig. 6.5A). The apparent path of least action proceeds from the folded state through a metastable state labeled by the representative frame from cluster 17 (Rep. 17A), which is of interest be- 

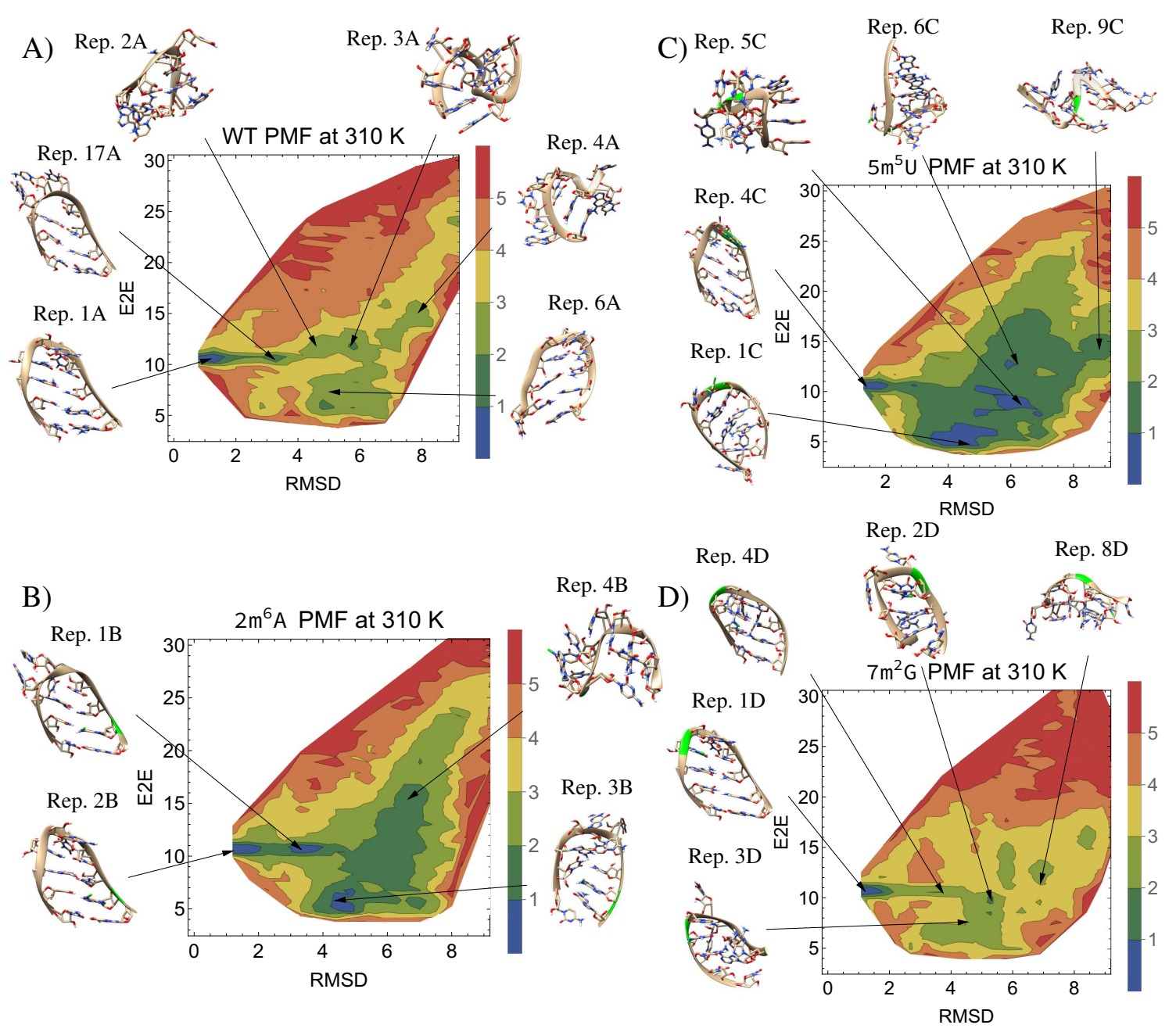

Figure 6.5: 2D PMFs and representative frames from hairpin TREMD simulations. The representative frames and PMFs for the A) WT B) modification of $2 \mathrm{~A}$ to $\mathrm{m}^{6} \mathrm{~A}\left(2 \mathrm{~m}^{6} \mathrm{~A}\right)$, C) modification of $5 \mathrm{U}$ to $\mathrm{m}^{5} \mathrm{U}\left(5 \mathrm{~m}^{5} \mathrm{U}\right)$, D) modification of $7 \mathrm{G}$ to $\mathrm{m}^{2} \mathrm{G}\left(7 \mathrm{~m}^{2} \mathrm{G}\right)$ are shown along the end-to-end distance (E2E) and RMSD reaction coordinates. Modified carbons and ribbons in the representative frames are highlighted in green, and the figure legends color code the energy in $\mathrm{kcal} / \mathrm{mol}$. 
cause of its deepening or disappearance in the PMFs of the methylated derivatives. The representative structure from this metastable cluster indicates that unfolding of the hairpin tends to proceed from the loop, and evaluation of the representative frame indicates that disruption of the tetraloop structure is caused by disruption of the noncanonical, UG trans sugar-edge/Watson-Crick base pair between $4 \mathrm{U}$ and $7 \mathrm{G}$ and formation of single-stranded stacking interactions within the loop. After reaching the metastable cluster, the path of least action appears to proceed through clusters 2 and 3, respectively labeled as Rep. 2A and Rep. 3A, which are characterized by single stranded stacking interactions. After reaching cluster 3 , the path of least action diverges, leading either to a set of collapsed conformations, characterized by a low E2E distance due to terminal nucleotide stacking (Rep. 6A), or extended states, characterized by a larger E2E distance (Rep. 4A). The greatest energy barrier between the $\mathrm{NH}$ and random coil (RC) states is about $2.8 \mathrm{kcal} / \mathrm{mol}$ and occurs after the metastable state is reached. Swapping $9 \mathrm{U}$ to form noncanonical AG, AC, or AA base pairs resulted in total disruption of the hairpin and disappearance of the $\mathrm{NH}$ from the energy landscape by $50 \mathrm{~ns}$.

In comparison to the unmodified WT, $\mathrm{N}^{6}$-methyladenosine $\left(\mathrm{m}^{6} \mathrm{~A}\right)$ methylation of nucleotide 2A ( $\left.2 \mathrm{~m}^{6} \mathrm{~A}\right)$ stabilizes the metastable intermediate (Rep. 1B) between the hairpin and the RC conformations (see Fig. 6.5B). The energy barrier separating NH and RC states is lowered to about $1.7 \mathrm{kcal} / \mathrm{mol}$, facilitating the transition. Furthermore, the $2 \mathrm{~m}^{6} \mathrm{~A}$ RNA PMF shows that this methylation stabilizes stacking in a collapsed conformation, where the terminal nucleotides and the methylated nucleotide together form a three nucleotide stack (Rep. 3B). The destabilization of the folded hairpin resulting from this methylation can be visualized in the PMF, where more low energy paths can be drawn from the folded state to a high E2E distance. While methylation at this position undoubtedly destabilizes 
the hairpin base pair, the representative frame from cluster 2 (Rep. 2B) shows the $\mathrm{NH}$ conformation is a long lived conformation. The overall destabilization is probably because this methylation stabilizes a wider range of extended and collapsed conformations through stacking, which can compete with the NH. In agreement with a previous study [175], we found that to promote base-pairing in a duplex, the methyl group must rotate into a higher energy anti conformation, whereas in an unpaired context, this group prefers the lower energy syn conformation. After calculating the average dipole moment for the simulated WT 2A base atoms ( $\mid$ dipole $\mid \sim 2.21 \pm 0.27 \mathrm{D}$ ) and $\mathrm{m}^{6} \mathrm{~A}$ in both a pairing and single-stranded contexts ( $\mid$ dipole $\mid \sim 2.33 \pm .17 \mathrm{D}$ ), it appears that this methylation enhances the magnitude of the dipole moment by $\sim 0.1 \mathrm{D}$ and also increases the stability of the dipole moment as measured by the standard deviation of the dipole moment by $\sim 0.1 \mathrm{D}$, causing the dipole moment to remain closer to the base plane with fewer fluctuations, regardless of whether the methyl group is in the syn or anti conformation. Because most of the base atoms are constrained by the aromaticity of the purine rings, the WT 2A dipole moment fluctuations are largely driven by the orientation of the $\mathrm{N}^{6} \mathrm{H}_{2}$ hydrogen atoms, which can freely rotate around the nitrogen. When the amino group is methylated, the base dipole moment cannot fluctuate as freely because the methyl group is more sterically constrained, and the methylenhanced stability and strength of the dipole moment may contribute to the base stacking stability. The duplex destabilization and single stranded stacking stabilization caused by $\mathrm{m}^{6} \mathrm{~A}$ modification gives rise to a multitude of regulatory capabilities for this modification, including mRNA stability, splicing, and translation [166]. Excessive suppression and upregulation of $\mathrm{m}^{6} \mathrm{~A}$ are both associated with disease [160-162, 164-168]. This modification is the most common mRNA modification, and the relatively modest effect of $\mathrm{m}^{6} \mathrm{~A}$ on the energy landscape shown here can have profound biological effects by delicately controlling 
pri-miRNA duplex stability and efficacy of dicer enzyme cleavage. Further, modification of mature miRNA can likely regulate the balance of available mRNA for translation by adjusting miRNA-mRNA duplex stability.

When $5 \mathrm{U}$ is methylated to ribothymidine $\left(5 \mathrm{~m}^{5} \mathrm{U}\right)$, the hairpin structure is greatly destabilized by enhanced stability of stacking interactions with other nucleotides (see Fig. 6.5C). In the WT NH (Rep. 1A), 5U engages in no obvious interactions with other nucleotides and is pointed towards solution on the minor groove side of the helix with the fifth carbon of the WT NH 5U located near the phosphate backbone in the NH structure. Methylating this ring carbon pushes the nucleotide away from the backbone and forces the hairpin into a higher energy conformation, which promotes sampling of alternative structures. The greatest energy barrier between $\mathrm{NH}$ and $\mathrm{RC}$ states occurs between the $\mathrm{NH}$ and the metastable state and is lowered to about $1.5 \mathrm{kcal} / \mathrm{mol}$. The minor groove has a dearth of stacking opportunities, but once $\mathrm{m}^{5} \mathrm{U}$ finds its way to the major groove, it stabilizes coil structures through stacking interactions, which naturally disrupts the NH conformation (Rep. 4C) since the location of $5 \mathrm{U}$ on the minor groove side is required to form the $\mathrm{NH}$ structure. Additionally, this methylation stabilizes the metastable state (Rep. 17A in Fig. 6.5A shows that $5 \mathrm{U}$ engages in single stranded stacking with $6 \mathrm{C}$ ), which facilitates the transformation from folded hairpin to the RC conformation. The promotion of stacking interactions with other nucleotides by $5 \mathrm{~m}^{5} \mathrm{U}$ that explains the tendency for this hairpin to misfold into collapsed coil conformations can be seen in Reps. 1C, 5C, 6C, and 9C in Fig 6.5C. These results indicate that methylation of $5 \mathrm{U}$ in the common, stable UUCG tetraloop weakens the stability of the tetraloop and may lead to disruption of the associated stem, which could be used as a tool to facilitate RNA nanotechnology by allowing the designer to avoid formation of particular tetraloops and off-target structures. Supporting the assessment that 
this modification tends to destabilize the folded structure, we estimate that the relative free energy change due to $5 \mathrm{~m} 5 \mathrm{U}$ modification is $\Delta \Delta G=0.7 \mathrm{kcal} / \mathrm{mol}$.

Unlike most other methylations, $\mathrm{N}^{2}$-methylguanosine $\left(\mathrm{m}^{2} \mathrm{G}\right)$ modification of $7 \mathrm{G}\left(7 \mathrm{~m}^{2} \mathrm{G}\right)$ markedly stabilizes Rep. 1D, the NH conformation (see Fig. 6.5D). The energy barrier separating the $\mathrm{NH}$ and $\mathrm{RC}$ states is about $2.8 \mathrm{kcal} / \mathrm{mol}$, similar to the WT PMF. Mechanistically, this methylation stabilizes the stacking interaction between $7 \mathrm{G}$ and $8 \mathrm{G}$ without greatly inhibiting base pair formation with $4 \mathrm{U}$, which partially explains the resulting PMF. In addition, many conformations that stabilize RC conformations of the WT, including the metastable conformation found in cluster 17 of Fig. 6.5A (Rep. 17A), use the $\mathrm{N}^{2}$ functional group of $7 \mathrm{G}$ to occupy a pocket formed by $6 \mathrm{C}$ and the backbone. This methylation reduces the ability of the molecule to form the pocket in the metastable state (Rep. 4D), which results in a relative energetic destabilization of $\mathrm{RC}$ conformations (Reps. 2D, 3D, and 8D). In short, this methylation stabilizes the tetraloop structure without causing instabilities in the rest of the hairpin and destabilizes the metastable state and RC states, which causes it to prefer the $\mathrm{NH}$ structure. These results indicate that methylation of the loop-closing $7 \mathrm{G}$ to $\mathrm{m}^{2} \mathrm{G}$ in this common tetraloop further stabilizes the loop and stem of the hairpin, which could also be used in RNA design. We estimate that the relative free energy change due to $7 \mathrm{~m}^{2} \mathrm{G}$ modification is $\Delta \Delta G=0.75 \mathrm{kcal} / \mathrm{mol}$. While the modifications discussed above provide a nice summary of our results, we also analyzed other methylations in varying sequence contexts. These modifications show a broad spectrum of stabilizing and destabilizing effects with $\Delta \Delta G$ ranging from -0.75 to $0.72 \mathrm{kcal} / \mathrm{mol}$. Each modification has a unique impact on the ruggedness of the PMF and on the stability of the structures in the ensemble. Most modifications have a notable impact on the energy barrier separating the $\mathrm{NH}$ and RC states, providing some potential insights into the kinetic changes driven by 
these modifications.

\subsection{Conclusion}

Due to the rapid development of our knowledge about the impact of RNA nucleotide modification on human health, studies of the underlying mechanics that lead to the emergence of diseases or disorders relating to nucleotide modification are increasingly in demand. In this study, we use computational modeling of structures with modified nucleotides to reproduce experimental evidence regarding the relative free energy changes that occur upon modification. First, we compared results from alchemical simulations of a duplex to thermal denaturation data, finding good agreement. Next, we established that no major deformations occur to the duplex topology after the perturbations, and we find small structural changes that point toward mechanisms that drive the free energy calculations. Then, we evaluated the effect of modification on the PMF of an analogous hairpin with a phylogenetically abundant UUCG tetraloop. Beyond a destabilizing limit, we show that the relative free energy changes extracted from hairpin PMFs reach a plateau that can be identified as an estimate of the absolute free energy of the hairpin. Furthermore, we elucidate the underlying modification-dependent mechanisms that cause alterations to the PMF for a common RNA hairpin motif. Information from this study may be useful in therapeutic design of RNA structures. The method may be used to aid in design of local features in RNA structures for RNA nanotechnology $[68,203]$. Furthermore, the results presented here may be useful in understanding duplex or tetraloop stabilization changes that occur due to RNA nucleotide modifications, which when imbalanced may give rise to neurological disease, metastatic cancer, and other chronic conditions [60, 61, 160-170]. 
Both alchemical and TREMD simulation results indicate that we can accurately reproduce the experimental results for $\mathrm{m}^{6} \mathrm{~A}$ modification, which implies that the force field parameterization for this modification is sufficient to obtain structural insights allowing for accurate study of the role of this modification in the mechanisms of epilepsy pathogenesis in silico. Here, we found that this modification destabilizes the duplex structure and stabilizes collapsed, RC conformations in a hairpin. The result here may explain the experimental finding that modification of $\mathrm{A}$ to $\mathrm{m}^{6} \mathrm{~A}$ in the seed region of miRNA-134 and miRNA-146 would likely affect the maturation and targeting ability of the miRNA structures. Similarly, we found that other simple modifications, such as $\mathrm{m}^{2} \mathrm{G}$ and $\mathrm{m}^{5} \mathrm{U}$, can strikingly stabilize or destabilize the hairpin structure in specific contexts, which may provide a tool for RNA nanotherapeutic design. Taking all of these context-dependent affects of modification together, this work further demonstrates the need for more experimental data of modified nucleotides in varying sequence contexts. 


\section{Appendix A}

\section{Appendix to Quantitative Understanding of SHAPE Mechanism from RNA Structure and Dynamics Analysis}

\section{A.1 Visualizing the bound SHAPE ligand}

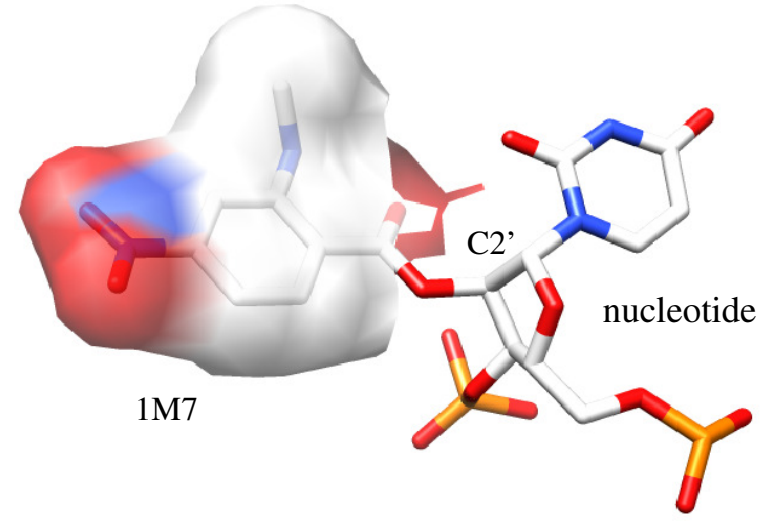

Figure A.1: The 1M7-nucleotide bound conformation, built based on two similar ligands in the PDB database (PubChem CID 12076 and 66345). The effective ligand radius is measured using the Van der Waals surface shown in the figure. 


\section{A.2 Leave-one-out parameter optimization}

Table A.1: Parameter optimization using the leave-one-out approach. $E_{s t}$ and $b$ are in $\mathrm{kcal} / \mathrm{mol}, S_{0}$ is in $\AA^{2}$, and $a$ is dimensionless.

\begin{tabular}{lccccc}
\hline \hline Value of $a$ & Value of $b$ & Value of $E_{s t}$ & Value of $S_{0}$ & Training set & Optimal total correlation \\
\hline 0.03 & -0.80 & -0.20 & 16 & without 3DIG & 7.53 \\
0.03 & -0.45 & -0.20 & 17 & without 1Y26 & 7.29 \\
0.03 & -0.40 & -0.25 & 16 & without 2GDI & 7.26 \\
0.03 & -0.50 & -0.25 & 14 & without 3IWN & 7.48 \\
0.03 & -0.48 & -0.28 & 14 & without 1NBS & 7.61 \\
0.03 & -0.60 & -0.25 & 15 & without 4KQY & 7.45 \\
0.03 & -0.55 & -0.25 & 15 & without 2L8H & 7.20 \\
0.03 & -0.33 & -0.25 & 22 & without 2L1V & 7.46 \\
0.03 & -0.50 & -0.20 & 15 & without 3PDR & 7.58 \\
0.03 & -0.45 & -0.25 & 14 & without 1VTQ & 7.48 \\
0.03 & -0.48 & -0.25 & 16 & without 1EHZ & 7.42 \\
0.03 & -0.43 & -0.25 & 12 & without 1AUD & 7.31 \\
\hline \hline
\end{tabular}




\section{A.3 Experimental conditions of SHAPE experiments}

Table A.2: Experimental conditions of the SHAPE chemical probing for twelve RNAs.

\begin{tabular}{|c|c|c|c|c|}
\hline Source name & PDB ID & Reagent & Solution conditions & Ref. \\
\hline $\begin{array}{l}\text { Lysine riboswitch, } \\
\text { T. maritime }\end{array}$ & 3DIG & $1 \mathrm{M} 7$ & $\begin{array}{l}\text { 100mM HEPES, } 100 \mathrm{mM} \mathrm{NaCl} \text {, } \\
10 \mathrm{mM} \mathrm{MgCl}_{2}, 310 \mathrm{~K}\end{array}$ & {$[2]$} \\
\hline adenine riboswitch & $1 Y 26$ & $1 \mathrm{M} 7$ & $\begin{array}{l}50 \mathrm{mM} \mathrm{HEPES}^{2}, 200 \mathrm{mM} \text { KOAc, } \\
3 \mathrm{mM} \mathrm{MgCl}_{2}, 298 \mathrm{~K}\end{array}$ & [1] \\
\hline TPP riboswitch, E. coli & 2GDI & $1 \mathrm{M} 7$ & $\begin{array}{l}\text { 100mM HEPES, } 100 \mathrm{mM} \mathrm{NaCl} \text {, } \\
10 \mathrm{mM} \mathrm{MgCl}_{2}, 310 \mathrm{~K}\end{array}$ & {$[2]$} \\
\hline $\begin{array}{l}\text { cyclic-di-GMP riboswitch, } \\
\text { V. cholerae }\end{array}$ & $3 I W N$ & $1 \mathrm{M} 7$ & $\begin{array}{l}\text { 50mM HEPES, 200mM KoAc, } \\
3 \mathrm{mM} \mathrm{MgCl}_{2}, 310 \mathrm{~K}\end{array}$ & {$[2]$} \\
\hline $\begin{array}{l}\text { SAM I riboswitch, } \\
\text { T. tengcongensis }\end{array}$ & 4KQY & $1 \mathrm{M} 7$ & $\begin{array}{l}\text { 100mM HEPES, } 100 \mathrm{mM} \mathrm{NaCl} \text {, } \\
10 \mathrm{mM} \mathrm{MgCl}_{2}, 310 \mathrm{~K}\end{array}$ & {$[2]$} \\
\hline RNaseP specificity domain & $1 \mathrm{NBS}$ & $1 \mathrm{M} 7$ & $\begin{array}{l}50 \mathrm{mM} \mathrm{HEPES}, 200 \mathrm{mM} \text { KOAc, } \\
3 \mathrm{mM} \mathrm{MgCl}_{2}, 298 \mathrm{~K}\end{array}$ & {$[1]$} \\
\hline TAR RNA from HIV-1 & $2 \mathrm{~L} 8 \mathrm{H}$ & $1 \mathrm{M} 7$ & $\begin{array}{l}50 \mathrm{mM} \text { HEPES, } 50 \mathrm{mM} \mathrm{KCl} \text {, } \\
298 \mathrm{~K}\end{array}$ & [3] \\
\hline Pre-Q1 riboswitch & $2 \mathrm{~L} 1 \mathrm{~V}$ & $1 \mathrm{M} 7$ & $\begin{array}{l}100 \mathrm{mM} \text { HEPES, } 100 \mathrm{mM} \mathrm{NaCl} \text {, } \\
10 \mathrm{mM} \mathrm{MgCl}, 310 \mathrm{~K}\end{array}$ & {$[2]$} \\
\hline M-Box riboswitch & 3PDR & $1 \mathrm{M} 7$ & $\begin{array}{l}100 \mathrm{mM} \text { HEPES, } 100 \mathrm{mM} \mathrm{NaCl} \text {, } \\
10 \mathrm{mM} \mathrm{MgCl}, 310 \mathrm{~K}\end{array}$ & {$[2]$} \\
\hline tRNAasp & 1VTQ & $1 \mathrm{M} 7$ & $\begin{array}{l}100 \mathrm{mM} \text { HEPES, } 100 \mathrm{mM} \mathrm{NaCl} \text {, } \\
10 \mathrm{mM} \mathrm{MgCl}, 310 \mathrm{~K}\end{array}$ & {$[2]$} \\
\hline tRNAphe & $1 \mathrm{EHZ}$ & $1 \mathrm{M} 7$ & $\begin{array}{l}100 \mathrm{mM} \text { HEPES, } 100 \mathrm{mM} \mathrm{NaCl} \text {, } \\
10 \mathrm{mM} \mathrm{MgCl}, 310 \mathrm{~K}\end{array}$ & [4] \\
\hline $\begin{array}{l}\text { U1A protein binding } \\
\text { site RNA }\end{array}$ & 1AUD & $1 \mathrm{M} 7$ & $\begin{array}{l}50 \mathrm{mM} \text { HEPES, } 50 \mathrm{mM} \mathrm{KCl} \text {, } \\
298 \mathrm{~K}\end{array}$ & {$[3]$} \\
\hline
\end{tabular}




\section{A.4 Tabulating native and decoy $2 \mathrm{D}$ structures}


Table A.3: Sequences, native 2D, and non-native 2D structures.

\begin{tabular}{|c|c|c|}
\hline PDB ID & Label & Structure \\
\hline \multirow[t]{7}{*}{ 1AUD } & Sequence & GGCAGAGUCCUUCGGGACAUUGCACCUGCC \\
\hline & Native & $(((((.((((\ldots)))) \ldots \ldots)))))$. \\
\hline & Non-native 1 &.$\left(\left(\left(\left(\left(_{(}(((\ldots)))\right).\right)\right)\right)\right) \ldots \ldots$ \\
\hline & Non-native 2 & $\ldots \ldots((((\ldots)))) \ldots((\ldots))$. \\
\hline & Non-native 3 & 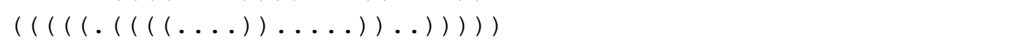 \\
\hline & Non-native 4 & $(((((.((\ldots \ldots((\ldots(\ldots)))))))))))$ \\
\hline & Non-native 5 & $((\ldots((((\ldots))))).) \ldots \ldots \ldots$ \\
\hline \multirow[t]{7}{*}{ 1EHZ } & Sequence & $\begin{array}{l}\text { GCGGAUUUAGCUCAGUUGGGAGAGCGCCAGACUGAAGAUCUGGAGGUCCUGUGUUCGAUC } \\
\text { CACAGAAUUCGCACCA }\end{array}$ \\
\hline & Native & 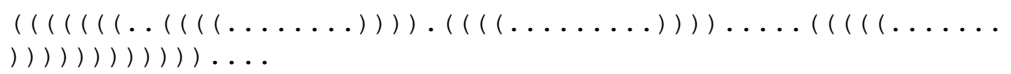 \\
\hline & Non-native 1 & 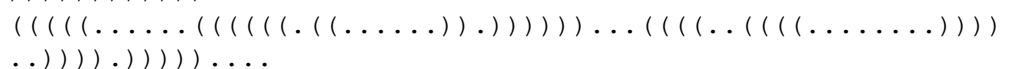 \\
\hline & Non-native 2 & 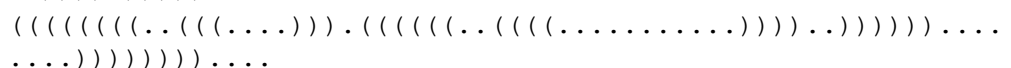 \\
\hline & Non-native 3 & $\left(\left(\left(\left(\left(\left(\left((\ldots)((((((.((\ldots \ldots))))))).) \cdot\left(\left(\left(\left(.\left(\left(\left(.(\ldots)^{\prime}\right)\right)\right)\right)\right)\right)\right)\right.\right.\right.\right.\right.\right.\right.$ \\
\hline & Non-native 4 & 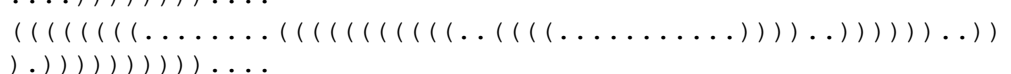 \\
\hline & Non-native 5 & 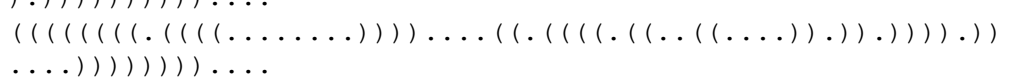 \\
\hline \multirow[t]{7}{*}{ 1NBS } & Sequence & $\begin{array}{l}\text { GCGAGCCUAGCGAAGUCAUAAGCUAGGGCAGUCUUUAGAGGCUGACGGCAGGAAAAAAGC } \\
\text { CUACGUCUUCGGAUAUGGCUGAGUAUCCUUGAAAGUGCCACAGUGACGAAGUCUCACUAG } \\
\text { AAAUGGUGAGAGUGGAACGCGGUAAACCCCUCGC }\end{array}$ \\
\hline & Native & 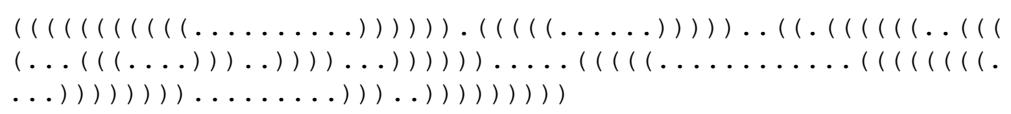 \\
\hline & Non-native 1 & 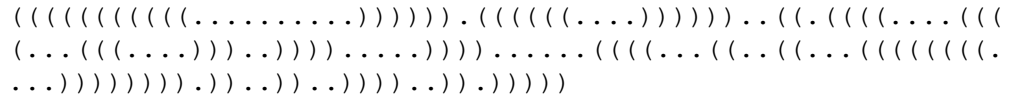 \\
\hline & Non-native 2 & 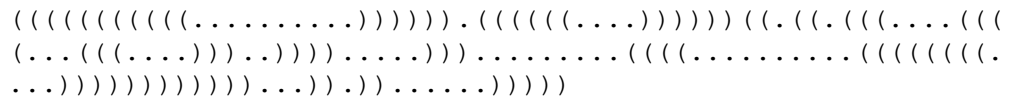 \\
\hline & Non-native 3 & 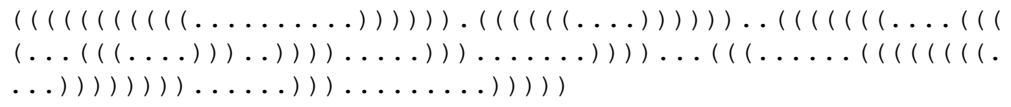 \\
\hline & Non-native 4 & 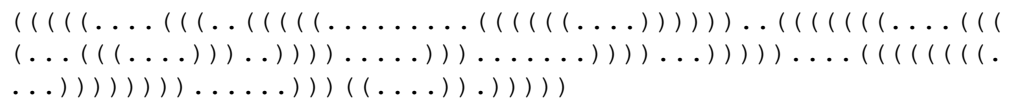 \\
\hline & Non-native 5 & 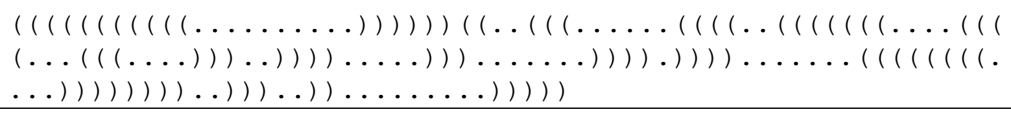 \\
\hline
\end{tabular}


Table A.4: Sequences, native 2D, and non-native 2D structures.

\begin{tabular}{|c|c|c|}
\hline PDB ID & Label & Structure \\
\hline TVTQ & $\begin{array}{l}\text { Sequence } \\
\text { Native } \\
\text { Non-native } 1 \\
\text { Non-native } 2 \\
\text { Non-native } 3 \\
\text { Non-native } 4 \\
\text { Non-native } 5\end{array}$ & 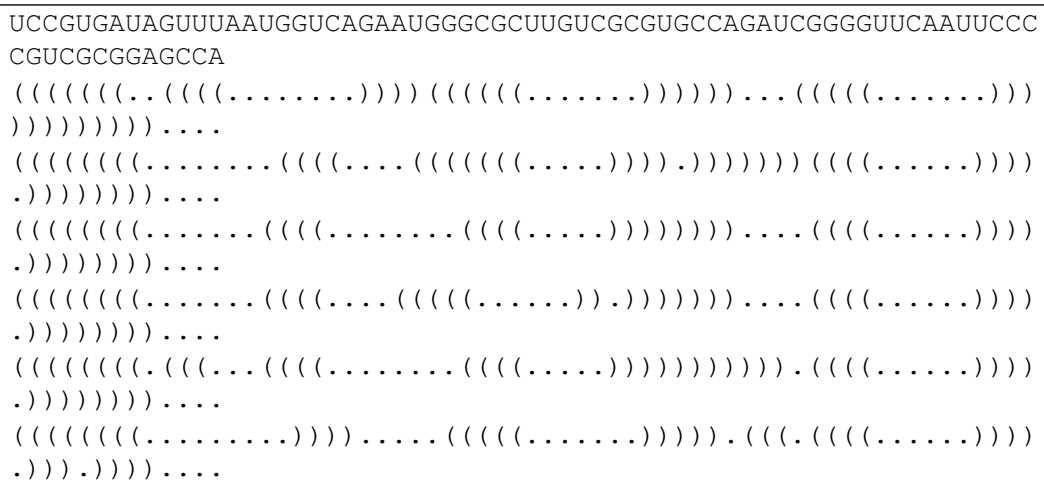 \\
\hline $1 Y 26$ & $\begin{array}{l}\text { Sequence } \\
\text { Native } \\
\text { Non-native } 1 \\
\text { Non-native } 2 \\
\text { Non-native } 3 \\
\text { Non-native } 4 \\
\text { Non-native } 5\end{array}$ & 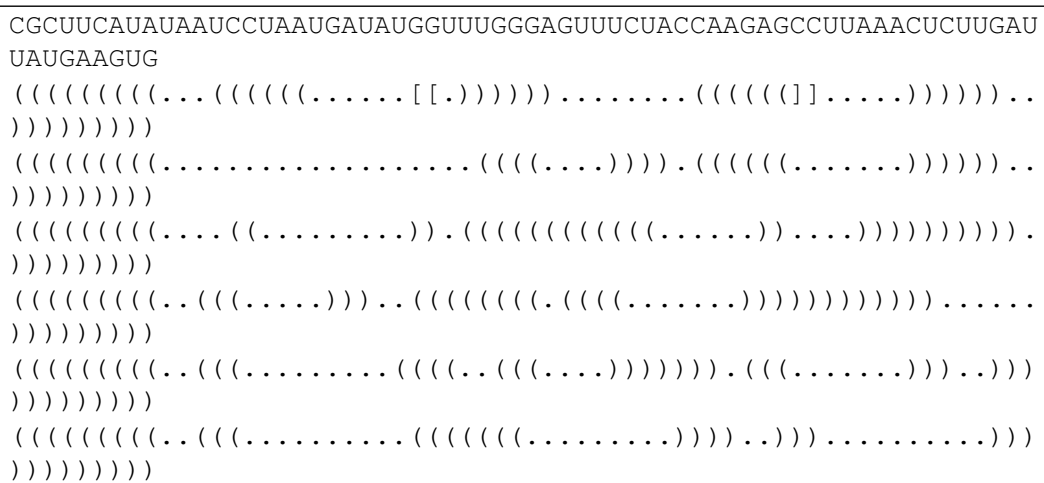 \\
\hline 2GDI & $\begin{array}{l}\text { Sequence } \\
\text { Native } \\
\text { Non-native } 1 \\
\text { Non-native } 2 \\
\text { Non-native } 3 \\
\text { Non-native } 4 \\
\text { Non-native } 5\end{array}$ & 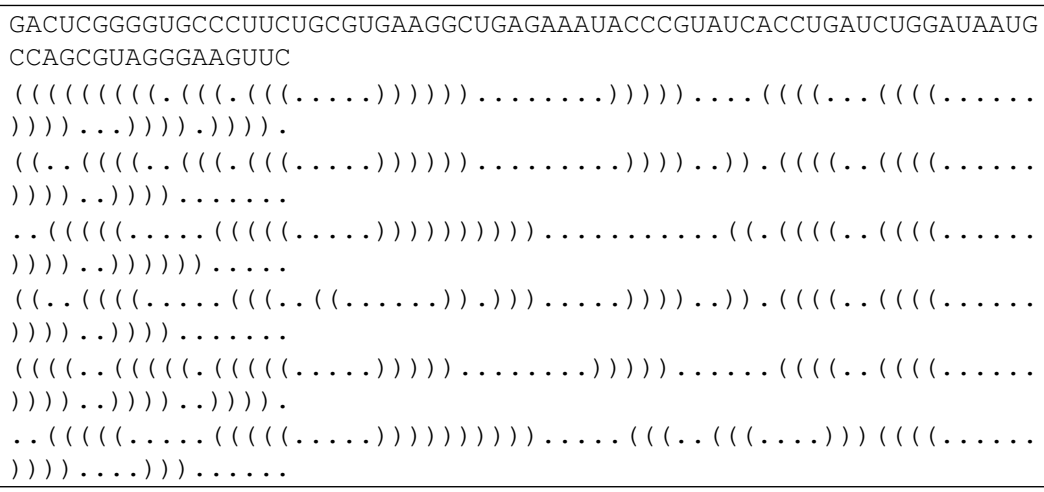 \\
\hline
\end{tabular}


Table A.5: Sequences, native 2D, and non-native 2D structures.

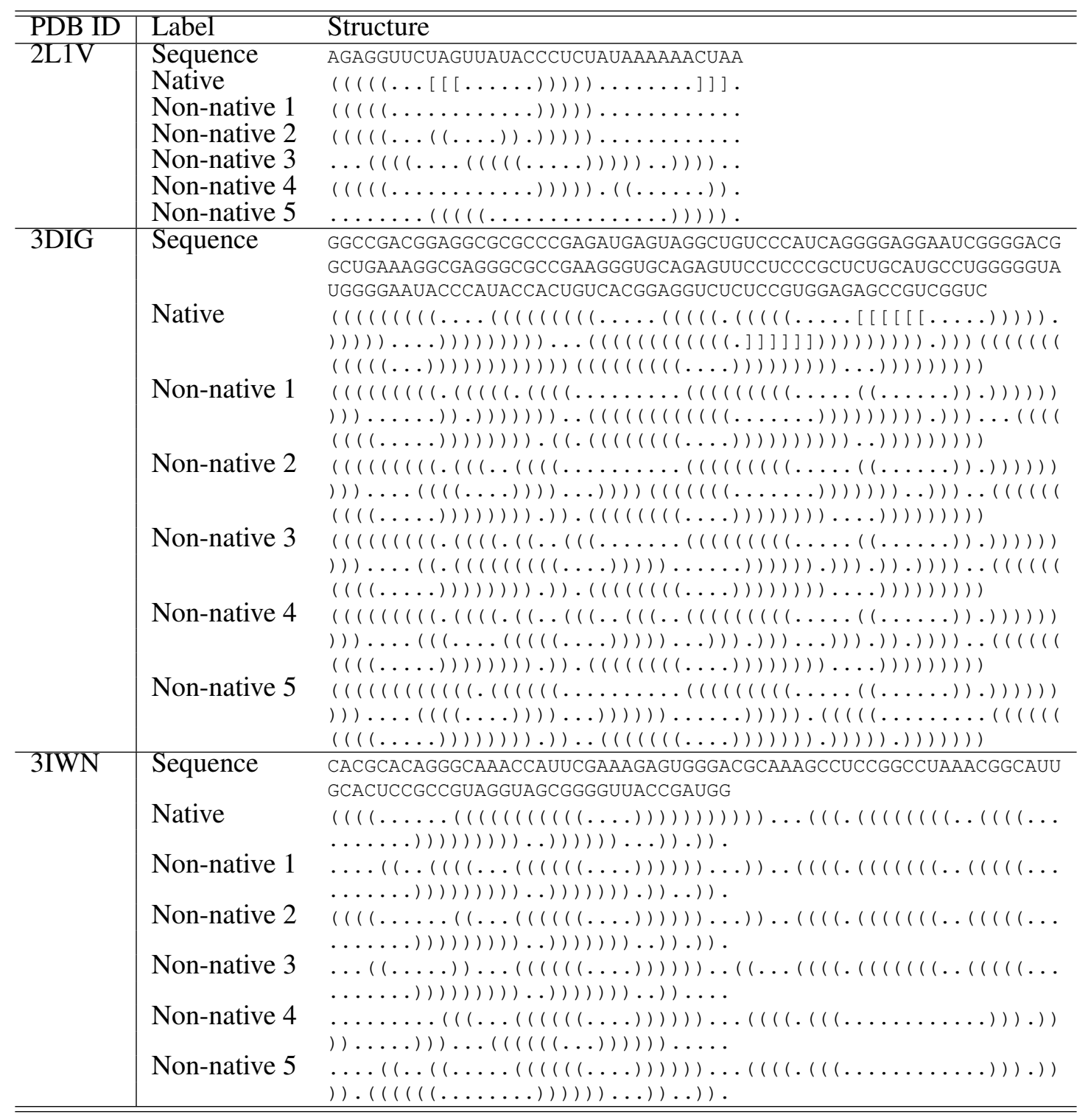


Table A.6: Sequences, native 2D, and non-native 2D structures.

\begin{tabular}{|c|c|c|}
\hline PDB ID & Label & Structure \\
\hline \multirow[t]{7}{*}{$2 \mathrm{~L} 8 \mathrm{H}$} & Sequence & GGCAGAUCUGAGCCUGGGAGCUCUCUGCC \\
\hline & Native & $\left(\left(\left(\left(\left(\left(\ldots\left(\left(\left({ }^{\ldots} \ldots(\ldots)\right)\right)\right)\right)\right)\right)\right)\right)\right)$ \\
\hline & Non-native 1 & $((((((\ldots(((.((\ldots)))))))))))$ \\
\hline & Non-native 2 & $((((((((((\ldots \ldots \ldots))) \ldots \ldots))))))$ \\
\hline & Non-native 3 & $((((((((((\ldots \ldots))) \ldots \ldots))))))$ \\
\hline & Non-native 4 & $(((((((((((\ldots)))) \ldots \ldots))))))$ \\
\hline & Non-native 5 & $(((((((((\ldots \ldots \ldots)) \ldots))))))$ \\
\hline \multirow[t]{7}{*}{ 3PDR } & Sequence & $\begin{array}{l}\text { CUUCGUUAGGUGAGGCUCCUGUAUGGAGAUACGCUGCUGCCCAAAAAUGUCCAAAGACGC } \\
\text { CAAUGGGUCAACAGAAAUCAUCGACAUAAGGUGAUUUUUAAUGCAGCUGGAUGCUUGUCC } \\
\text { UAUGCCAUACAGUGCUAAAGCUCUACGAUUGAAG }\end{array}$ \\
\hline & Native & 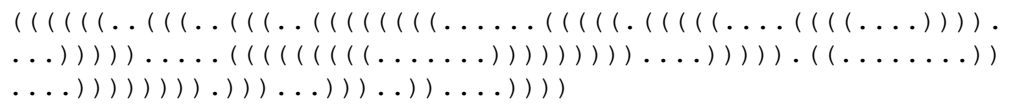 \\
\hline & Non-native 1 & 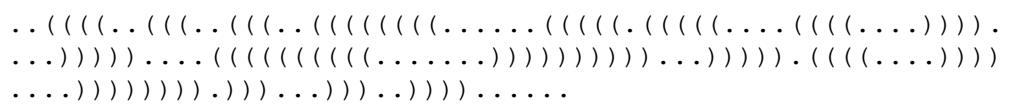 \\
\hline & Non-native 2 & 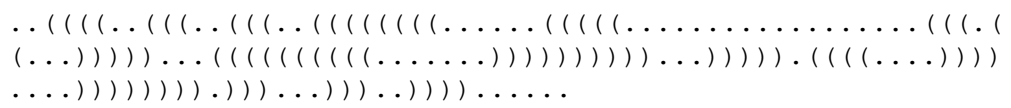 \\
\hline & Non-native 3 & 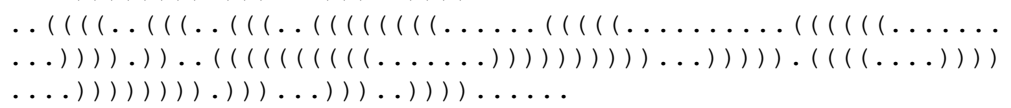 \\
\hline & Non-native 4 & 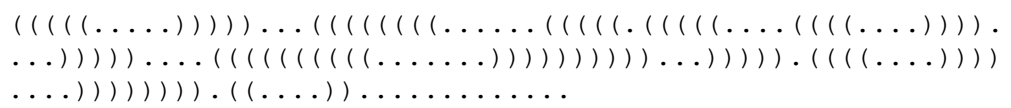 \\
\hline & Non-native 5 & 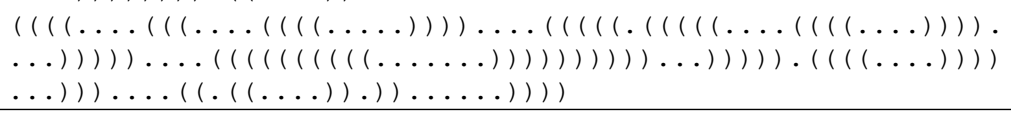 \\
\hline \multirow[t]{8}{*}{ 4KQY } & Sequence & $\begin{array}{l}\text { UUCUUAUCAAGAGAAGCAGAGGGACUGGCCCGACGAAGCUUCAGCAACCGGUGUAAUGGC } \\
\text { GAAAGCCAUGACCAAGGUGCUAAAUCCAGCAAGCUCGAACAGCUUGGAAGAUAAGAA }\end{array}$ \\
\hline & Native & 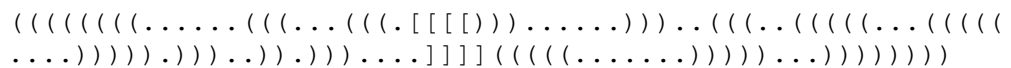 \\
\hline & Non-native 1 & 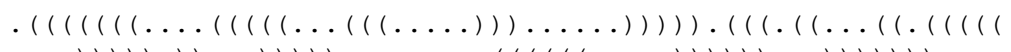 \\
\hline & Non-native 2 & 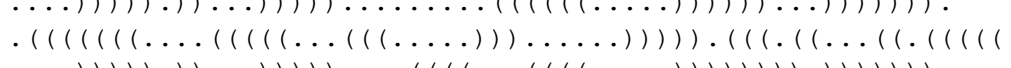 \\
\hline & Non-native 3 & 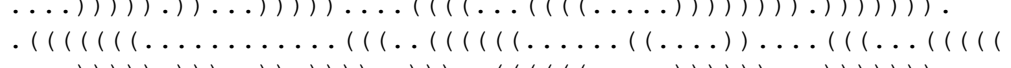 \\
\hline & Non-native 4 & 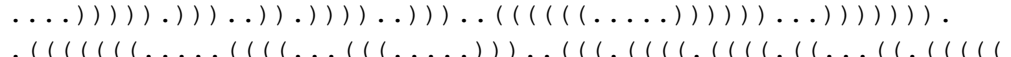 \\
\hline & & 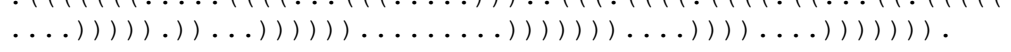 \\
\hline & Non-native 5 & 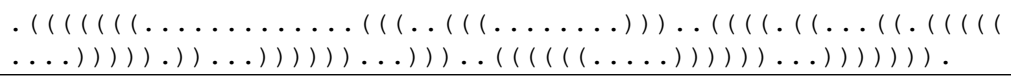 \\
\hline
\end{tabular}




\section{A.5 Evaluating performance of SHAPE predictions}
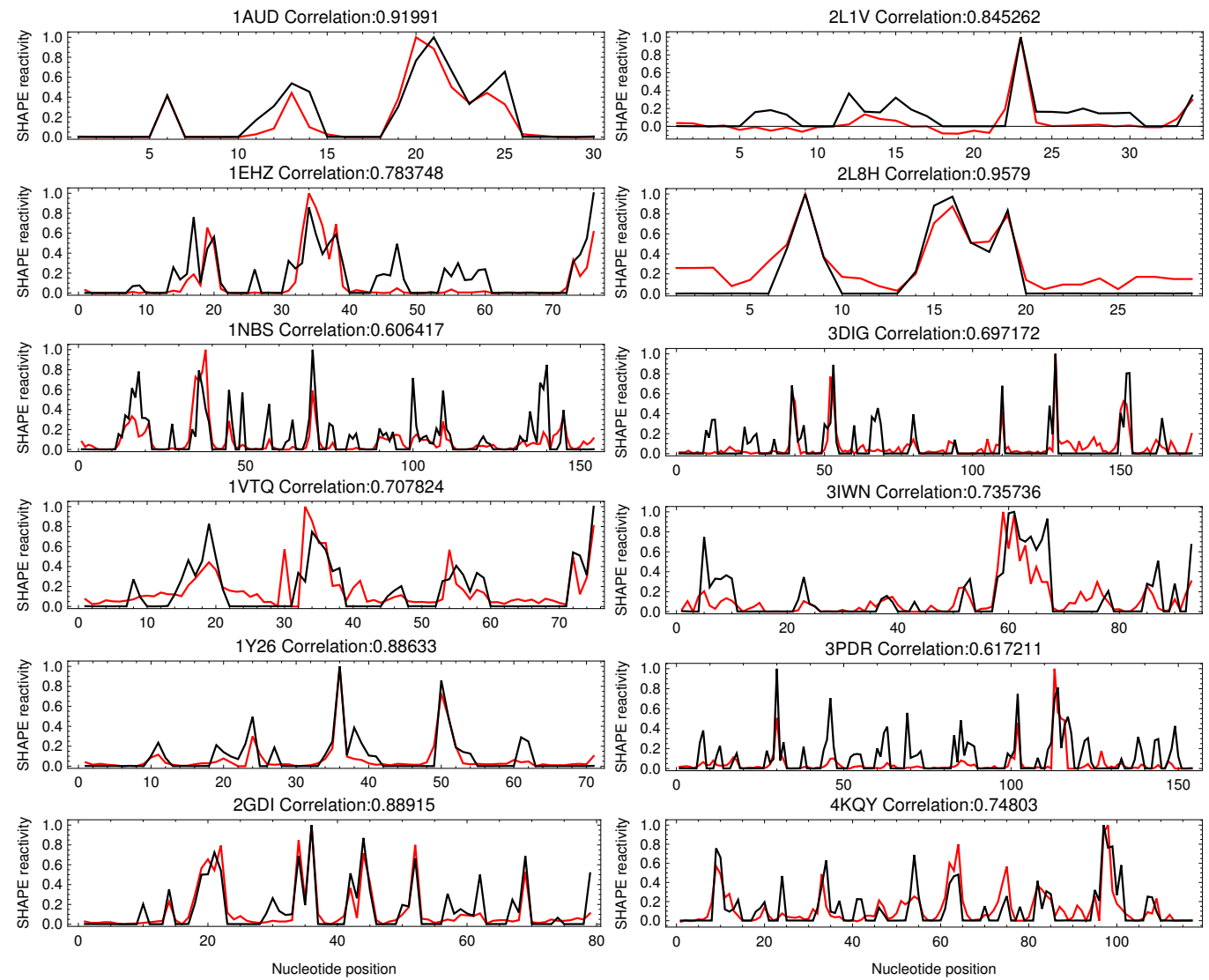

Figure A.2: Comparison between the predicted (black) and the experimental (red) SHAPE profiles. 

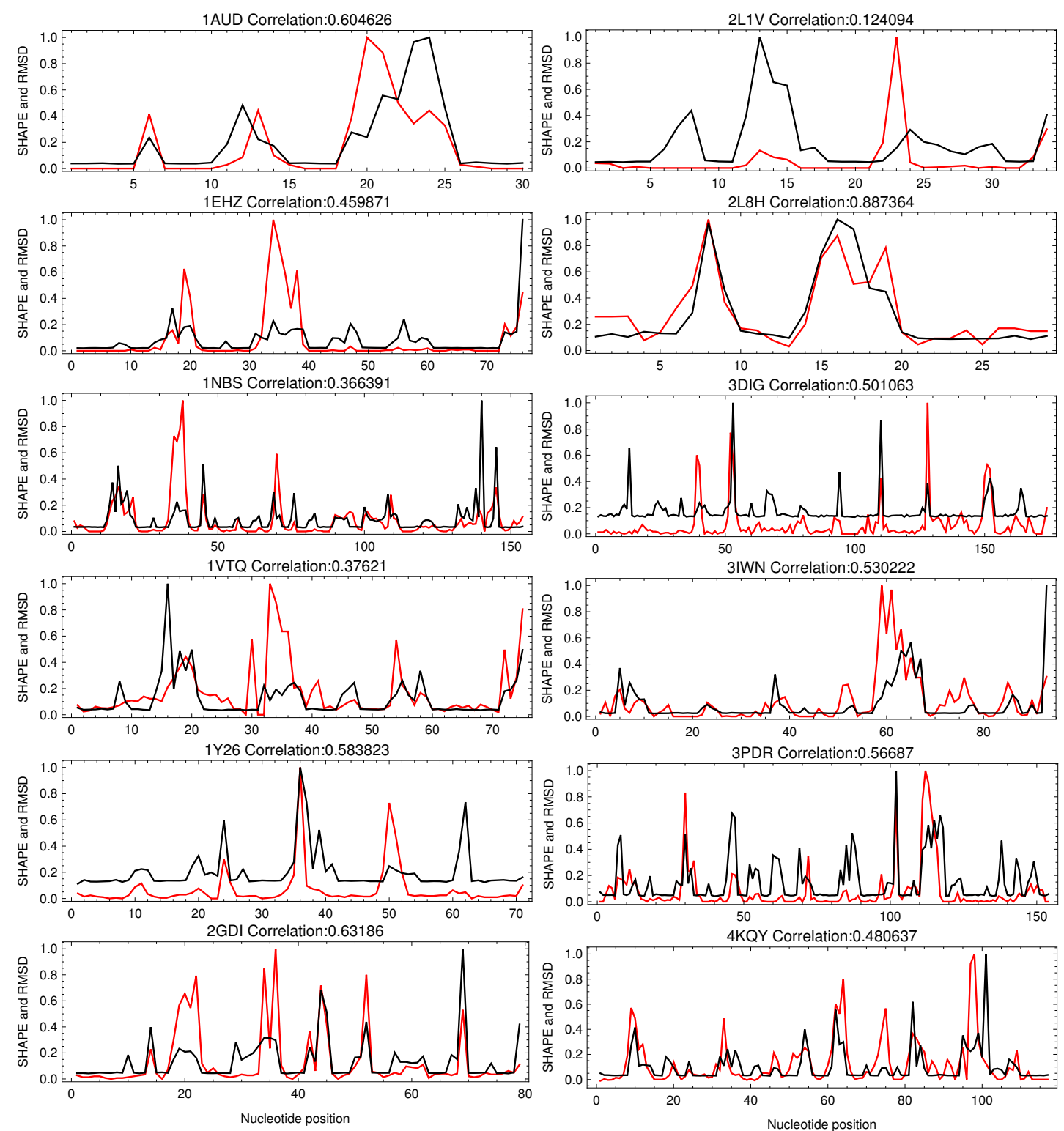

Figure A.3: Comparison between the nucleotide RMSD (black) and the experimental SHAPE (red) profiles. 

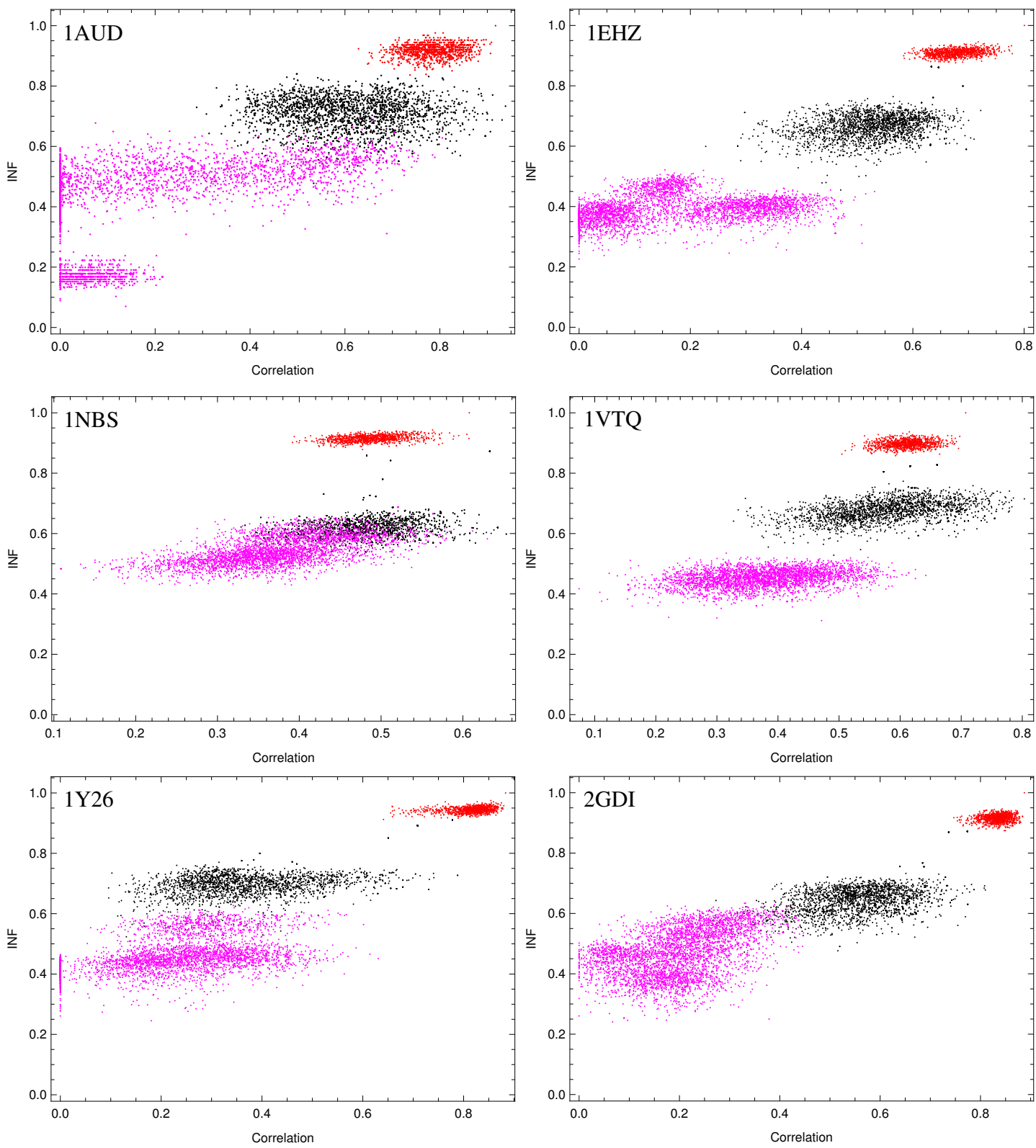

Figure A.4: Comparison of INF and correlation for the first six cases. Near-native structures from all-atom MD sampling are red; native 2D, non-native 3D structures from CG sampling are black; non-native $2 \mathrm{D}$, non-native $3 \mathrm{D}$ structures from $\mathrm{CG}$ sampling are magenta. 

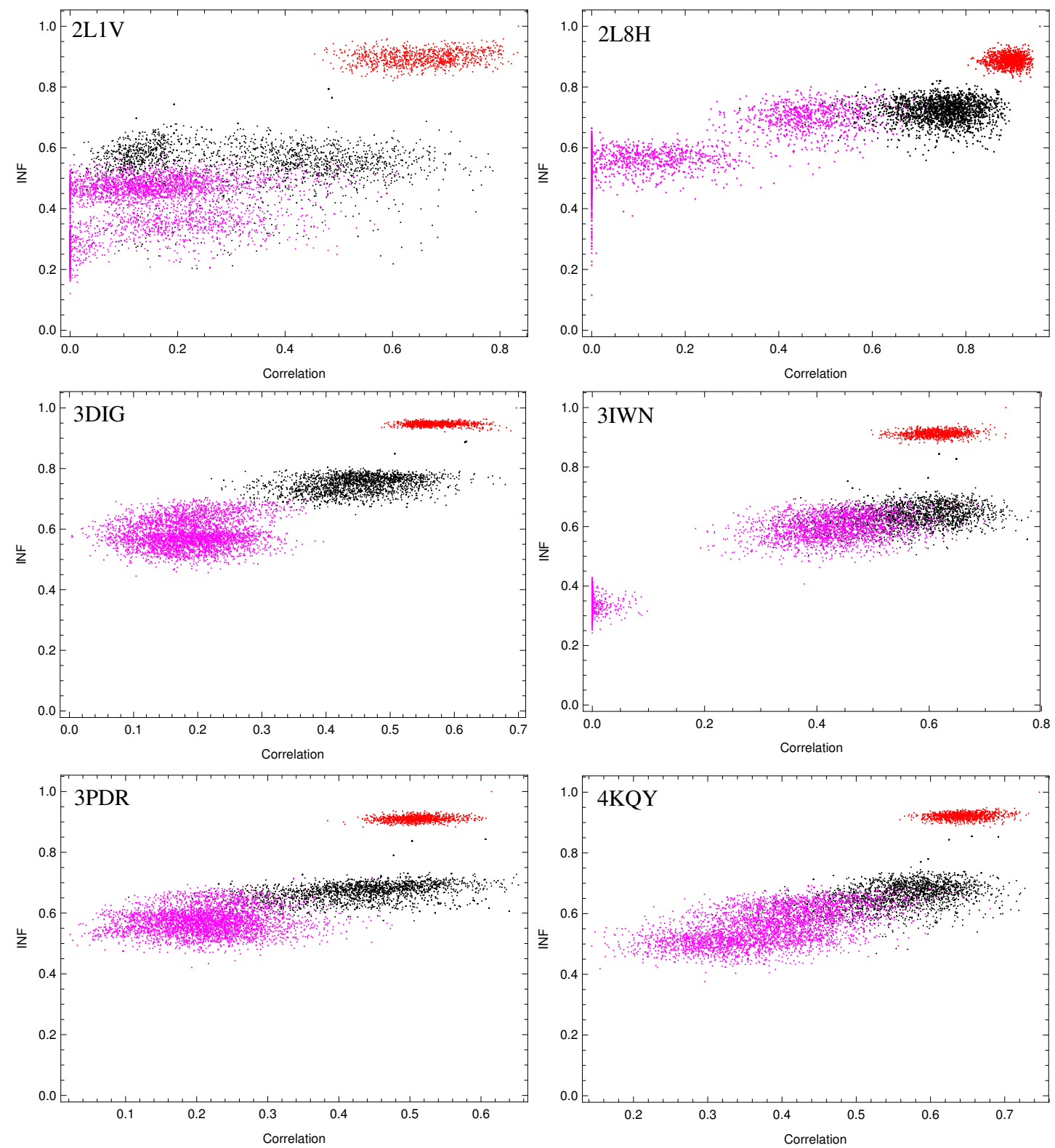

Figure A.5: Comparison of INF and correlation for the last six cases. Near-native structures from all-atom MD sampling are red; native 2D, non-native 3D structures from CG sampling are black; non-native $2 \mathrm{D}$, non-native $3 \mathrm{D}$ structures from $\mathrm{CG}$ sampling are magenta. 

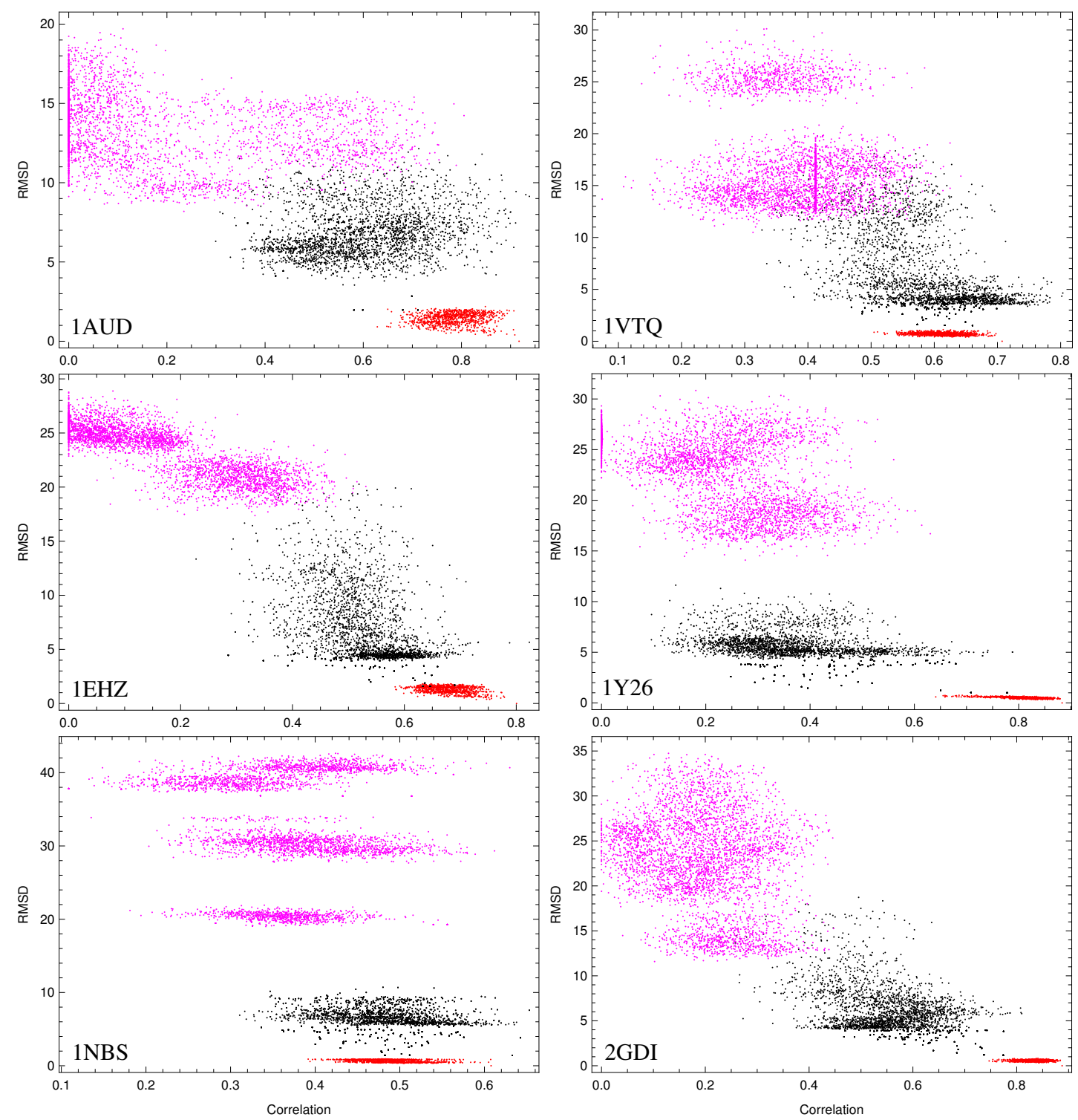

Figure A.6: Comparison of RMSD and correlation for the first six cases. Near-native structures from all-atom MD sampling are red; native 2D, non-native 3D structures from CG sampling are black; non-native 2D, non-native 3D structures from CG sampling are magenta. 

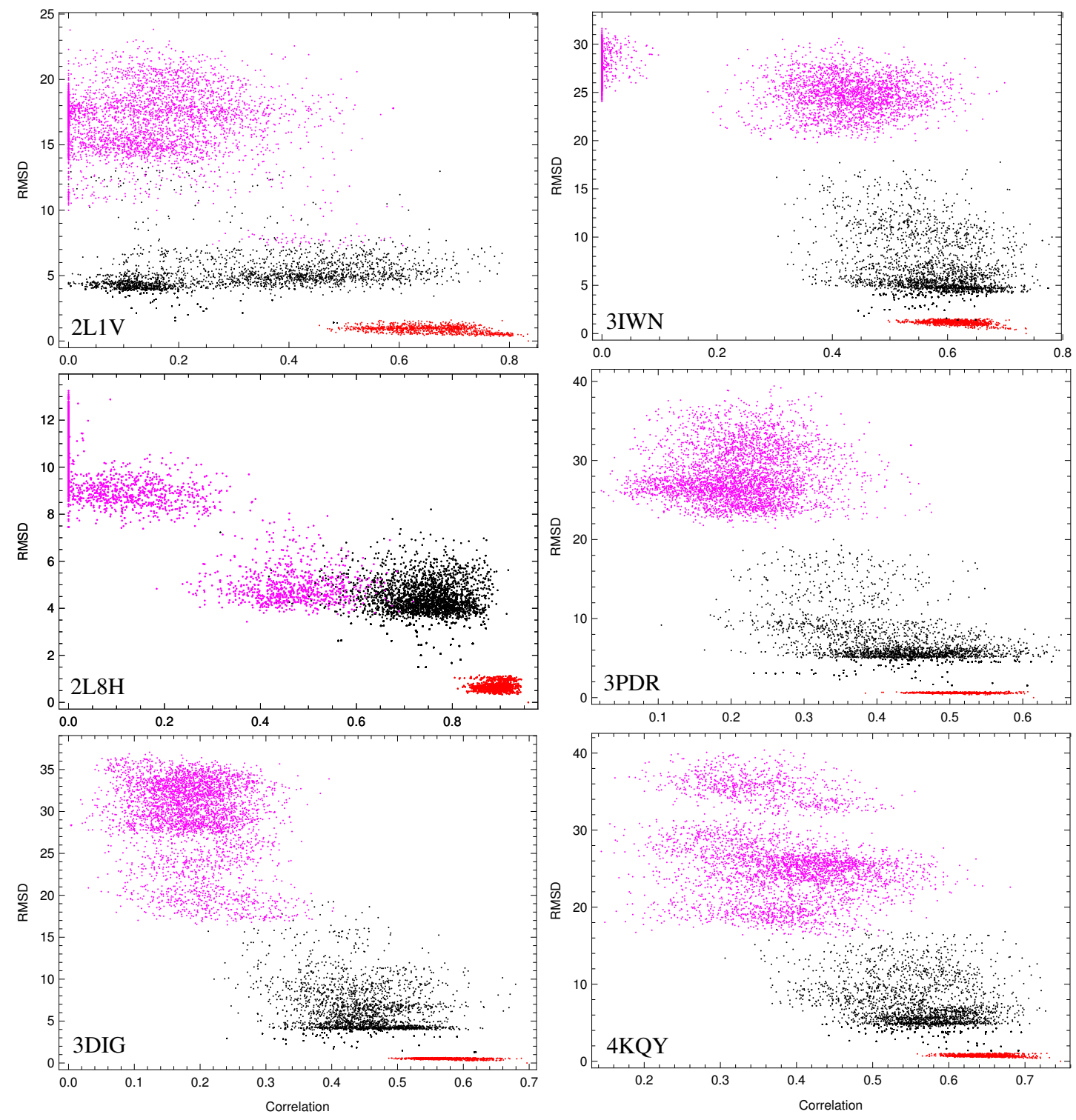

Figure A.7: Comparison of RMSD and correlation for the last six cases. Near-native structures from all-atom MD sampling are red; native 2D, non-native 3D structures from CG sampling are black; non-native 2D, non-native 3D structures from CG sampling are magenta. 


\section{Appendix B}

Appendix to Sieving RNA 3D Structures with SHAPE and Evaluating Mechanisms Driving Sequence-Dependent Reactivity Bias 


\section{B.1 Base pairing frequency table}

Table B.1: Table extracted from non-redundant structures in Basepair Catalog [129]. WatsonCrick, Hoogsteen, and sugar edges are denoted by W, H, and S, respectively. Cis and trans conformations are denoted by $\mathrm{c}$ and $\mathrm{t}$.

\begin{tabular}{lllllllllllll}
\hline Pair & cWW & tWW & cWH & tWH & cWS & tWS & cHH & tHH & cHS & tHS & cSS & tSS \\
\hline AA & 17 & 69 & 0 & 94 & 77 & 8 & 0 & 160 & 39 & 92 & 75 & 47 \\
UA & 4200 & 70 & 115 & 535 & 24 & 16 & 0 & 8 & 1 & 34 & 120 & 0 \\
GA & 191 & 0 & 6 & 0 & 3 & 0 & 4 & 5 & 3 & 0 & 94 & 4 \\
CA & 67 & 14 & 0 & 102 & 21 & 2 & 0 & 6 & 25 & 11 & 167 & 0 \\
AU & 4200 & 70 & 0 & 0 & 40 & 0 & 0 & 8 & 6 & 23 & 5 & 71 \\
UU & 216 & 5 & 15 & 16 & 6 & 2 & 0 & 0 & 18 & 0 & 2 & 0 \\
GU & 1144 & 4 & 0 & 8 & 11 & 64 & 0 & 0 & 0 & 0 & 17 & 4 \\
CU & 24 & 3 & 0 & 0 & 18 & 0 & 0 & 6 & 19 & 8 & 5 & 0 \\
AG & 191 & 0 & 4 & 6 & 1 & 140 & 4 & 5 & 6 & 822 & 119 & 467 \\
UG & 1144 & 4 & 8 & 1 & 12 & 9 & 0 & 0 & 135 & 30 & 34 & 0 \\
GG & 0 & 10 & 123 & 48 & 5 & 0 & 4 & 10 & 6 & 36 & 6 & 49 \\
CG & 9316 & 57 & 9 & 8 & 6 & 19 & 1 & 4 & 2 & 0 & 24 & 0 \\
AC & 67 & 14 & 0 & 0 & 87 & 3 & 0 & 6 & 6 & 31 & 275 & 133 \\
UC & 24 & 3 & 0 & 0 & 0 & 3 & 0 & 6 & 0 & 0 & 4 & 0 \\
GC & 9316 & 57 & 0 & 0 & 6 & 8 & 1 & 4 & 0 & 0 & 6 & 6 \\
CC & 6 & 7 & 4 & 8 & 8 & 9 & 0 & 0 & 5 & 13 & 0 & 0 \\
SUM & 15181 & 239 & 284 & 826 & 325 & 283 & 9 & 199 & 271 & 1100 & 953 & 781 \\
$\%$ & 74.23 & 1.17 & 1.39 & 4.04 & 1.59 & 1.38 & 0.04 & 0.97 & 1.33 & 5.38 & 4.66 & 3.82 \\
\hline
\end{tabular}




\section{B.2 Training parameters and 2D structures}

Table B.2: Physical descriptions for fit parameters in model.

\begin{tabular}{ll}
\hline \hline Parameters & Physical meaning and descriptions \\
\hline$A$ & Decomposition weight for combining base pairing with base stacking. \\
$B$ & Minimum energy for base pairing. \\
$C$ & Angle cutoff for stacking. \\
$D$ & Distance cutoff for stacking. \\
$A_{\mathrm{SAS}}^{0}$ & Accounts for possibility of nucleotide becoming solvent accessible. \\
$E_{2 D}$ & Energy for pairing in 2D structure. \\
$s_{0^{-}} s_{2}$ & Accounts for influence of neighbors on NOI reactivity in experiment. \\
$d_{0^{-}}-d_{3}$ & Accounts for influence of local 2D structure on NOI. \\
$a_{0^{-}} a_{3}$ & Accounts for influence of accessibility of neighbors. \\
$w_{0}-w_{3}$ & Accounts for tertiary rigidity (interaction strength) of nearby nucleotides. \\
$E_{5^{\prime}}$ & Energy contribution from the 5' stacking nucleotide on pairwise stacking interaction. \\
$E_{3^{\prime}}^{(A)}$ & Energy contribution from 3' adenosine on pairwise stacking interaction. \\
$E_{3^{\prime}}^{(U)}$ & Energy contribution from 3' uracil on pairwise stacking interaction. \\
$E_{3^{\prime}}^{(G)}$ & Energy contribution from 3' guanosine on pairwise stacking interaction. \\
$E_{3^{\prime}}^{(C)}$ & Energy contribution from 3' cytosine on pairwise stacking interaction. \\
$c_{0}-C_{3}$ & Decomposition weights for base stacking. \\
$F_{\text {term }}^{5^{\prime}}$ & Terminal contribution to reactivity for first three 5' -end nucleotides. \\
$F_{\text {term }}^{3^{\prime}}$ & Terminal contribution to reactivity for last two 3'-end nucleotides. \\
$F_{\text {sug }}^{\mathrm{C} 3 \mathrm{~N}}$ & Sugar conformation parameter for pseudorotation angles 0-144 and 324-360 degrees. \\
$F_{\mathrm{sug}}^{\mathrm{C} 3 \mathrm{X}}$ & Sugar conformation parameter for pseudorotation angles 180-216 degrees. \\
$F_{\mathrm{sug}}^{\mathrm{C} 4 \mathrm{~N}}$ & Sugar conformation parameter for pseudorotation angles 144-180 and 216-288 degrees. \\
$F_{\text {sug }}^{\mathrm{ClN}}$ & Sugar conformation parameter for pseudorotation angles 288-324 degrees. \\
$E_{\text {lig }}$ & Energy penalty for bound ligand contacts. \\
$\eta$ & Fit noise parameter. \\
\hline \hline & \\
\hline
\end{tabular}


Table B.3: Sequences, native 2D, and non-native 2D structures.

\begin{tabular}{|c|c|c|}
\hline PDB ID & Label & Structure \\
\hline \multirow[t]{7}{*}{ 1AUD } & Sequence & GGCAGAGUCCUUCGGGACAUUGCACCUGCC \\
\hline & Native & $(((((.((((\ldots))))) \ldots \ldots)))))$. \\
\hline & Non-native 1 & $((((((((((\ldots))))).) \ldots \ldots)))))$ \\
\hline & Non-native 2 & $(((((.((((\ldots)))) \ldots \ldots))))))$. \\
\hline & Non-native 3 & $((((\ldots((((\ldots)))) \ldots))) \ldots) \ldots$ \\
\hline & Non-native 4 & $(((((.((((\ldots))) \ldots \ldots)) \ldots)))))$ \\
\hline & Non-native 5 & $\ldots \ldots((((\ldots \ldots)))) \ldots((\ldots))$. \\
\hline \multirow[t]{7}{*}{$1 \mathrm{EHZ}$} & Sequence & $\begin{array}{l}\text { GCGGAUUUAGCUCAGUUGGGAGAGCGCCAGACUGAAGAUCUGGAGGUCCUGUGUUCGAUC } \\
\text { CACAGAAUUCGCACCA }\end{array}$ \\
\hline & Native & $\begin{array}{l}((((((()((((\ldots \ldots[\ldots)))) \cdot((((\ldots \ldots \ldots)))) \ldots \ldots(((((\ldots] \ldots \ldots \\
())))))))))) \ldots\end{array}$ \\
\hline & Non-native 1 & $\begin{array}{l}((((((\ldots)(((((\ldots((\ldots \ldots)) \ldots))))) \ldots((((\ldots((((\ldots \ldots \ldots)))) \\
\ldots())))))))) \ldots\end{array}$ \\
\hline & Non-native 2 & $\begin{array}{l}\left(\left(\left(((\ldots))\left(\left(\left(\left(\left((\ldots((\ldots \ldots))){ }^{\prime}\right)\right)\right)\right)\right)\right) \cdot((((\ldots((((\ldots \ldots \ldots))))\right.\right. \\
\ldots)))) .()))) \ldots \ldots\end{array}$ \\
\hline & Non-native 3 & $\begin{array}{l}(((((((() \ldots \ldots \ldots(((((((((((\ldots((((\ldots \ldots \ldots \ldots)))) \ldots)))))) \ldots)) \\
) .()))))))))) \ldots \ldots\end{array}$ \\
\hline & Non-native 4 & $\begin{array}{l}((((((\ldots \ldots)(((((\ldots((\ldots \ldots)) \ldots))))) \cdot((((\ldots .))))(((((\ldots \ldots \ldots \\
)))) .(\ldots)))) \ldots \ldots\end{array}$ \\
\hline & Non-native 5 & $\begin{array}{l}((((((\ldots \ldots)(((((\ldots((\ldots \ldots)) \ldots))))) \ldots((((\ldots((((\ldots(\ldots))))) \\
\ldots())))))))) \ldots\end{array}$ \\
\hline \multirow[t]{7}{*}{ 1NBS } & Sequence & $\begin{array}{l}\text { GCCUAGCGAAGUCAUAAGCUAGGGCAGUCUUUAGAGGCUGACGGCAGGAAAAAAGCCUAC } \\
\text { GUCUUCGGAUAUGGCUGAGUAUCCUUGAAAGUGCCACAGUGACGAAGUCUCACUAGAAAU } \\
\text { GGUGAGAGUGGAACGCGGUAAACCCCUCG }\end{array}$ \\
\hline & Native & 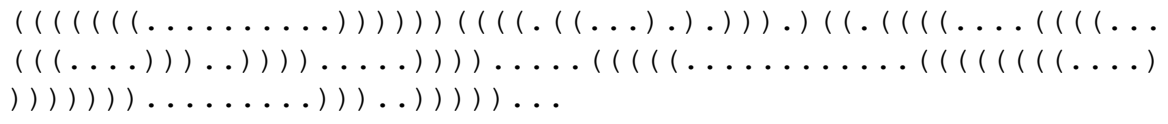 \\
\hline & Non-native 1 & 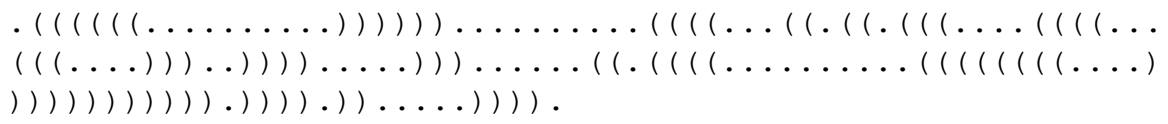 \\
\hline & Non-native 2 & 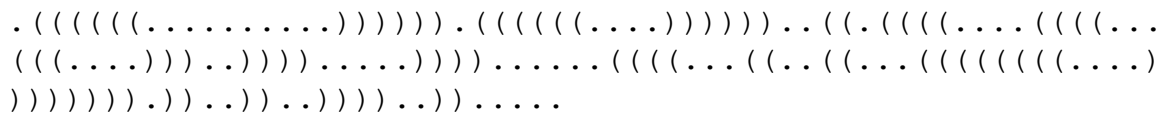 \\
\hline & Non-native 3 & 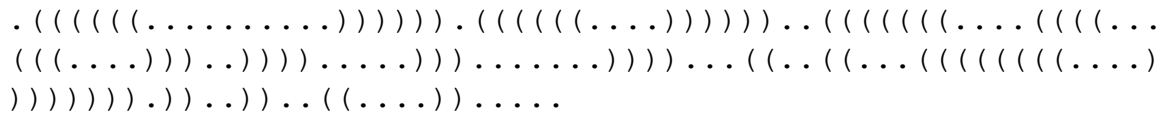 \\
\hline & Non-native 4 & 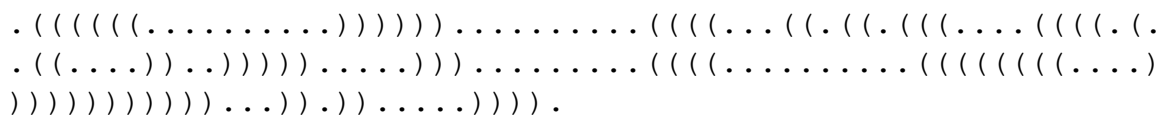 \\
\hline & Non-native 5 & 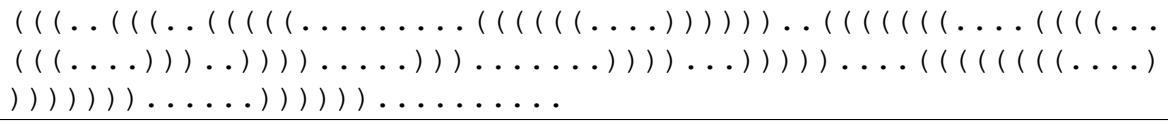 \\
\hline
\end{tabular}


Table B.4: Sequences, native 2D, and non-native 2D structures.

\begin{tabular}{|c|c|c|}
\hline PDB ID & Label & Structure \\
\hline \multirow[t]{7}{*}{ 1VTQ } & Sequence & $\begin{array}{l}\text { CCGUGAUAGUUUAAUGGUCAGAAUGGGCGCUUGUCGCGUGCCAGAUCGGGGUUCAAUUCC } \\
\text { CCGUCGCGGAGCCA }\end{array}$ \\
\hline & Native & $\begin{array}{l}((((((),(((\ldots \ldots \ldots \ldots))) \cdot(((((\ldots \ldots \ldots))))) \ldots \ldots(((((\ldots \ldots \ldots)) \\
))))))))) \ldots \ldots\end{array}$ \\
\hline & Non-native 1 & 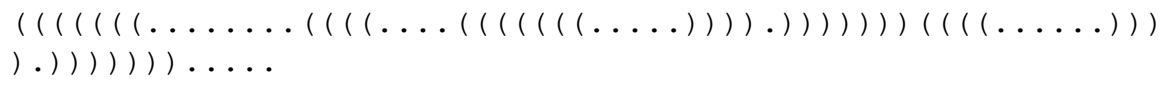 \\
\hline & Non-native 2 & $\begin{array}{l}((((((() \ldots \ldots \ldots \ldots \ldots((.(((((((\ldots \ldots)))) .))) \ldots))((((\ldots \ldots \ldots))) \\
())))))) \ldots \ldots\end{array}$ \\
\hline & Non-native 3 & $\begin{array}{l}((((((()((((\ldots \ldots \ldots)))) \cdot(((((\ldots \ldots))))) \ldots \ldots((((\ldots \ldots))) \\
) .())))))) \ldots \ldots\end{array}$ \\
\hline & Non-native 4 & $\begin{array}{l}((((((() \ldots \ldots((\ldots \ldots \ldots)) \cdot(((((\ldots \ldots \ldots))))) \ldots \ldots((((\ldots \ldots \ldots))) \\
\left.\left.\left.\left.\left.\left.()^{\prime}\right)\right)\right)\right)\right)\right) \ldots \ldots\end{array}$ \\
\hline & Non-native 5 & $\begin{array}{l}((((((),(((\ldots((((\ldots \ldots \ldots((((\ldots \ldots))))))))))) \cdot((((\ldots \ldots))) \\
) .))))))) \ldots \ldots\end{array}$ \\
\hline \multirow[t]{7}{*}{$1 \mathrm{Y} 26$} & Sequence & $\begin{array}{l}\text { CGCUUCAUAUAAUCCUAAUGAUAUGGUUUGGGAGUUUCUACCAAGAGCCUUAAACUCUUG } \\
\text { AUUAUGAAGUG }\end{array}$ \\
\hline & Native & $\begin{array}{l}((((((((() \ldots((((((\ldots \ldots \ldots[[.))))))[\ldots \ldots)](((((([\ldots \ldots)))))) \\
\ldots())))))))\end{array}$ \\
\hline & Non-native 1 & $\begin{array}{l}(((((((((\ldots)((((((\ldots \ldots \ldots \ldots)))))) \ldots \ldots \ldots((((((\ldots \ldots \ldots)))))) \\
\ldots()))))))))\end{array}$ \\
\hline & Non-native 2 & $\begin{array}{l}(((((((((\ldots)(((((\ldots(((\ldots)))))))) \ldots \ldots \ldots((((((\ldots \ldots)))))) \\
\ldots())))))))\end{array}$ \\
\hline & Non-native 3 & 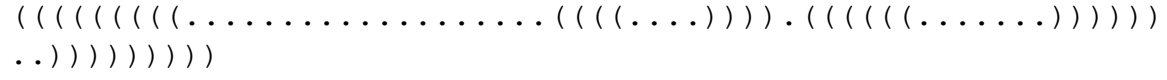 \\
\hline & Non-native 4 & $\begin{array}{l}((((((((() \ldots(((((((\ldots \ldots) \ldots)))))) \ldots \ldots \ldots((((((\ldots \ldots))))))) \\
\ldots())))))))\end{array}$ \\
\hline & Non-native 5 & 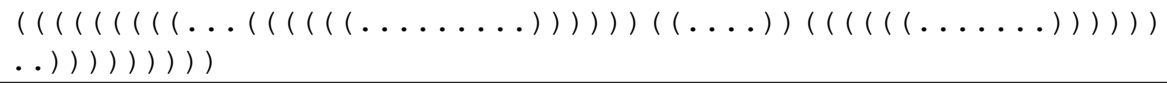 \\
\hline \multirow[t]{7}{*}{ 2GDI } & Sequence & $\begin{array}{l}\text { GACUCGGGGUGCCCUUCUGCGUGAAGGCUGAGAAAUACCCGUAUCACCUGAUCUGGAUAA } \\
\text { UGCCAGCGUAGGGAAGUU }\end{array}$ \\
\hline & Native & 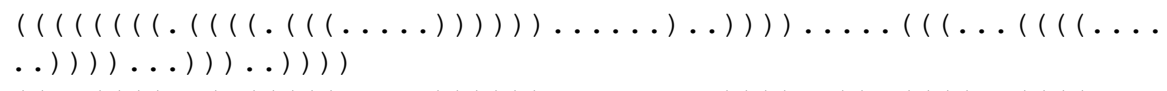 \\
\hline & Non-native 1 & 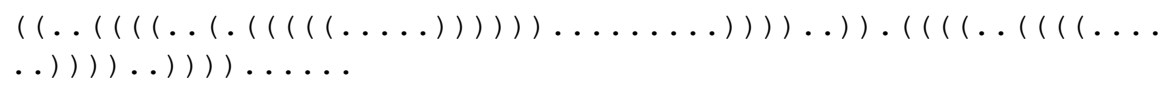 \\
\hline & Non-native 2 & 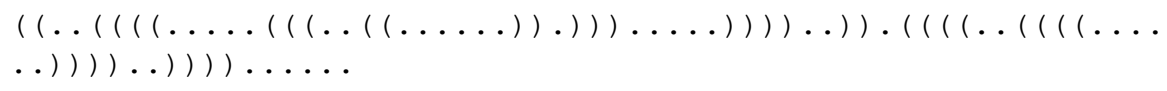 \\
\hline & Non-native 3 & 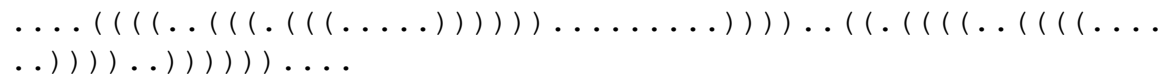 \\
\hline & Non-native 4 & $\begin{array}{l}((\ldots((((\ldots) \ldots((((((\ldots \ldots))) \ldots)))) \ldots)))) \ldots)) \cdot((((\ldots((((\ldots) \\
\ldots)))) \ldots())) \ldots \ldots\end{array}$ \\
\hline & Non-native 5 & 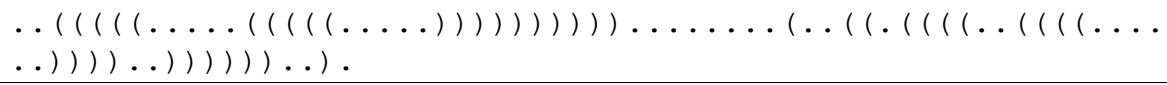 \\
\hline
\end{tabular}


Table B.5: Sequences, native 2D, and non-native 2D structures.

\begin{tabular}{|c|c|c|}
\hline PDB ID & Label & Structure \\
\hline \multirow[t]{7}{*}{ 2L1V } & Sequence & AGAGGUUCUAGUUAUACCCUCUAUAAAAAACUAA \\
\hline & Native & $(((((\ldots[[[\ldots \ldots))))) \ldots \ldots \ldots]]]$ \\
\hline & Non-native 1 & $(((((\ldots \ldots \ldots \ldots))))) \ldots \ldots \ldots$ \\
\hline & Non-native 2 & $(((((\ldots((\ldots)))))))) \ldots \ldots \ldots \ldots$ \\
\hline & Non-native 3 & 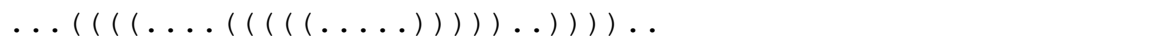 \\
\hline & Non-native 4 & $(((((\ldots \ldots \ldots \ldots))))) \cdot((\ldots \ldots))$. \\
\hline & Non-native 5 & $\ldots \ldots \ldots(((((\ldots \ldots \ldots \ldots \ldots \ldots)))))$ \\
\hline \multirow[t]{7}{*}{ 3DIG } & Sequence & $\begin{array}{l}\text { GGCCGACGGAGGCGCGCCCGAGAUGAGUAGGCUGUCCCAUCAGGGGAGGAAUCGGGGACG } \\
\text { GCUGAAAGGCGAGGGCGCCGAAGGGUGCAGAGUUCCUCCCGCUCUGCAUGCCUGGGGGUA } \\
\text { UGGGGAAUACCCAUACCACUGUCACGGAGGUCUCUCCGUGGAGAGCCGUCGGUC }\end{array}$ \\
\hline & Native & 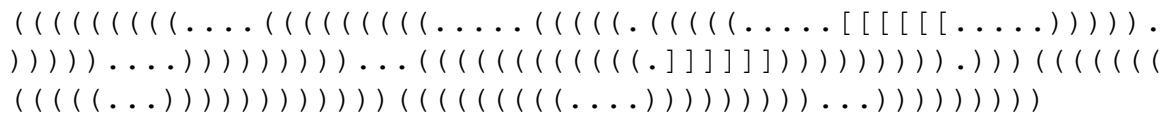 \\
\hline & Non-native 1 & 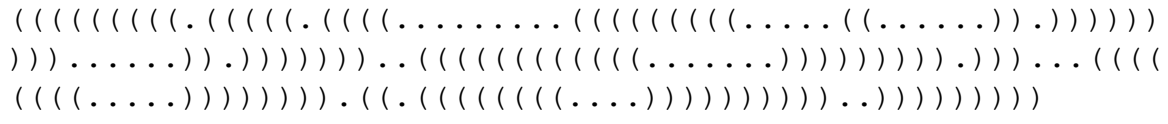 \\
\hline & Non-native 2 & 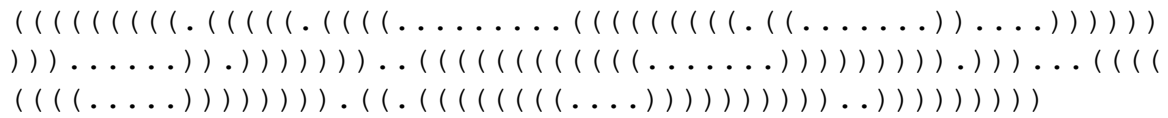 \\
\hline & Non-native 3 & 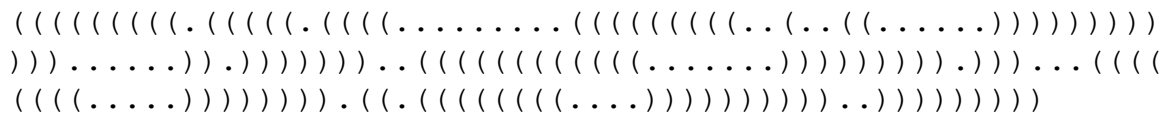 \\
\hline & Non-native 4 & 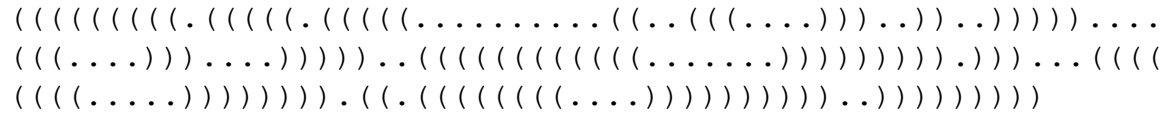 \\
\hline & Non-native 5 & 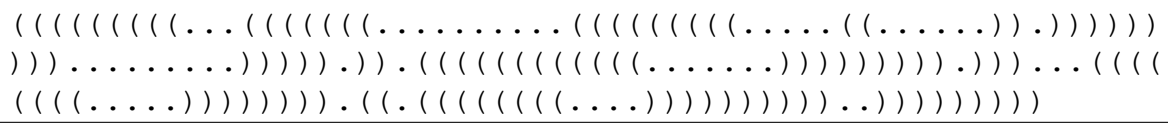 \\
\hline \multirow[t]{7}{*}{$3 \mathrm{IWN}$} & Sequence & $\begin{array}{l}\text { CACGCACAGGGCAAACCAUUCGAAAGAGUGGGACGCAAAGCCUCCGGCCUAAACGGCAUU } \\
\text { GCACUCCGCCGUAGGUAGCGGGGUUACCGAUGG }\end{array}$ \\
\hline & Native & 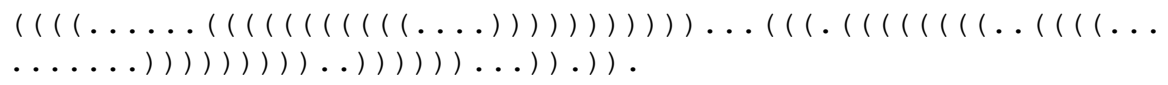 \\
\hline & Non-native 1 & 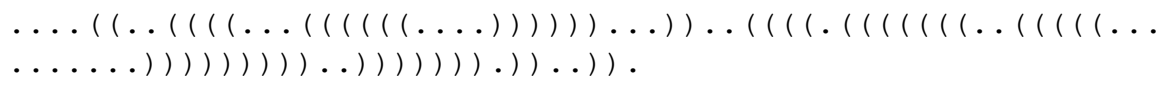 \\
\hline & Non-native 2 & 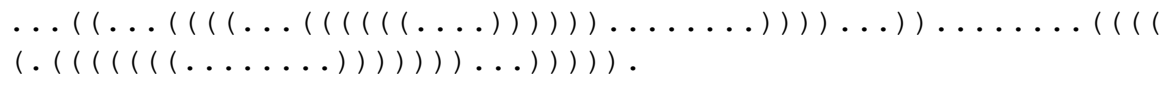 \\
\hline & Non-native 3 & 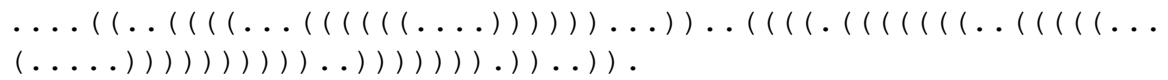 \\
\hline & Non-native 4 & 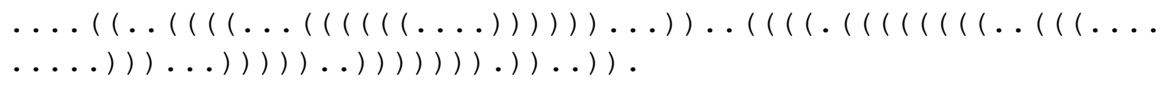 \\
\hline & Non-native 5 & 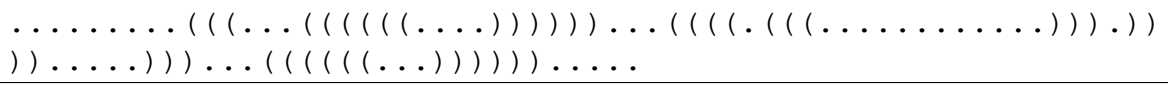 \\
\hline
\end{tabular}


Table B.6: Sequences, native 2D, and non-native 2D structures.

\begin{tabular}{|c|c|c|}
\hline PDB ID & Label & Structure \\
\hline \multirow[t]{7}{*}{$2 \mathrm{~L} 8 \mathrm{H}$} & Sequence & GGCAGAUCUGAGCCUGGGAGCUCUCUGCC \\
\hline & Native & $((((((\ldots((({ } \ldots \ldots))))))))))$ \\
\hline & Non-native 1 & $((((((\ldots(((\ldots((\ldots)))))))))))$ \\
\hline & Non-native 2 & $(((((((((\ldots \ldots)))) \ldots .))))))$. \\
\hline & Non-native 3 & $((((((\ldots \ldots \ldots \ldots((\ldots))))))))$ \\
\hline & Non-native 4 & $(((\ldots \ldots \ldots))) \cdot(((\ldots))) \ldots$ \\
\hline & Non-native 5 & $((((((.((\ldots \ldots \ldots)) \ldots))))))$ \\
\hline \multirow[t]{7}{*}{ 3PDR } & Sequence & $\begin{array}{l}\text { CUUCGUUAGGUGAGGCUCCUGUAUGGAGAUACGCUGCUGCCCAAAAAUGUCCAAAGACGC } \\
\text { CAAUGGGUCAACAGAAAUCAUCGACAUAAGGUGAUUUUUAAUGCAGCUGGAUGCUUGUCC } \\
\text { UAUGCCAUACAGUGCUAAAGCUCUACGAUUGAAG }\end{array}$ \\
\hline & Native & 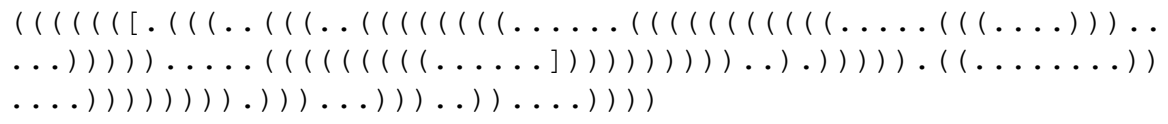 \\
\hline & Non-native 1 & 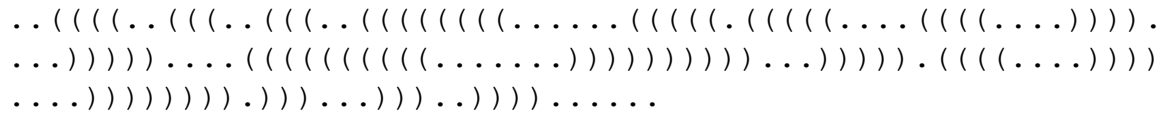 \\
\hline & Non-native 2 & 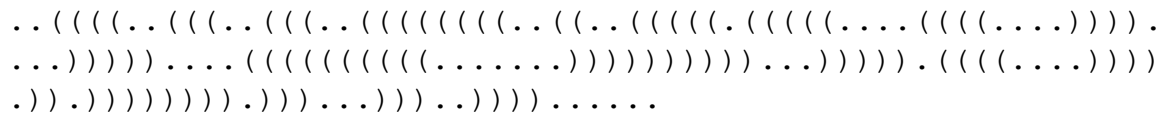 \\
\hline & Non-native 3 & 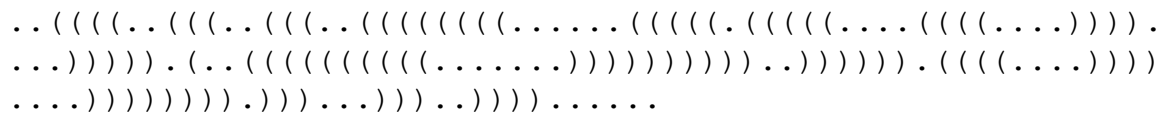 \\
\hline & Non-native 4 & 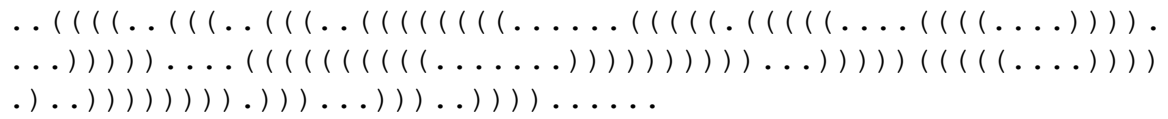 \\
\hline & Non-native 5 & 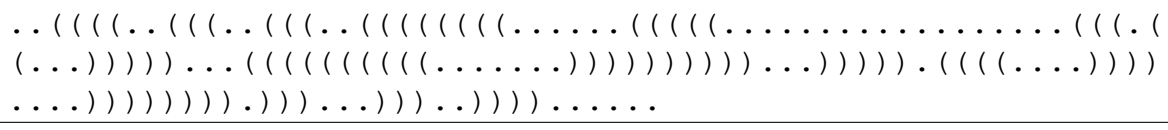 \\
\hline \multirow[t]{7}{*}{$4 \mathrm{KQY}$} & Sequence & $\begin{array}{l}\text { GUUCUUAUCAAGAGAAGCAGAGGGACUGGCCCGACGAAGCUUCAGCAACCGGUGUAAUGG } \\
\text { CGAAAGCCAUGACCAAGGUGCUAAAUCCAGCAAGCUCGAACAGCUUGGAAGAUAAGAA }\end{array}$ \\
\hline & Native & 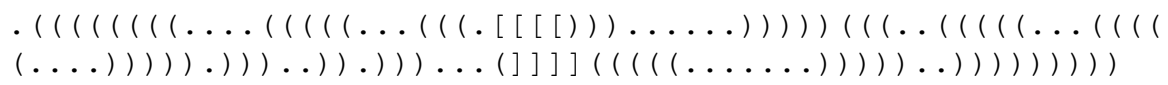 \\
\hline & Non-native 1 & 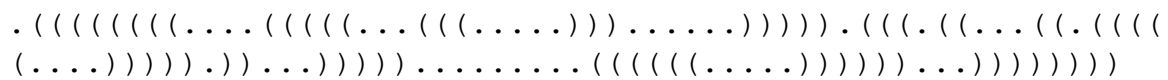 \\
\hline & Non-native 2 & 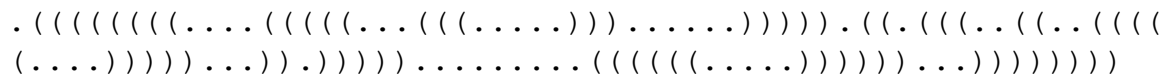 \\
\hline & Non-native 3 & 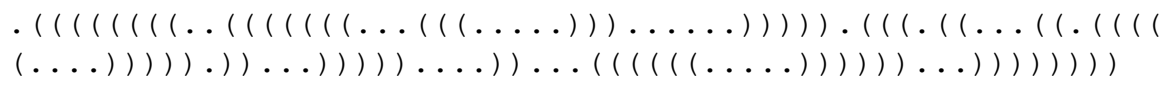 \\
\hline & Non-native 4 & 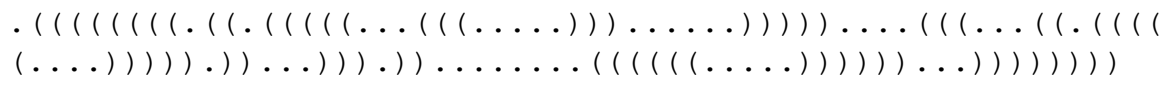 \\
\hline & Non-native 5 & 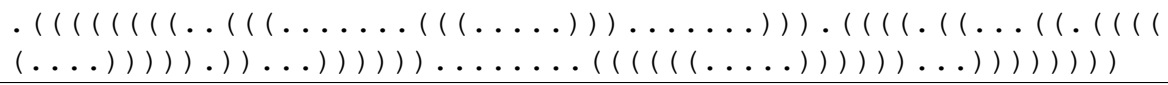 \\
\hline
\end{tabular}


Table B.7: Sequences, native 2D, and non-native 2D structures.

\begin{tabular}{|c|c|c|}
\hline PDB ID & Label & Structure \\
\hline \multirow[t]{7}{*}{$2 \mathrm{~K} 95$} & Sequence & GGGCUGUUUUUCUCGCUGACUUUCAGCCCCAAACAAAAAAUGUCAGC \\
\hline & Native & $\left.\left[\left[\left[\left[\left[\left[\ldots \ldots \ldots\left(\left(\left(\left(\left(\left(\left(()^{\prime}\right]\right]\right]\right]\right] \ldots \ldots \ldots\right)\right)\right).\right)\right)\right)\right)\right)\right)$ \\
\hline & Non-native 1 & $((((((\ldots \ldots((\ldots \ldots)) \ldots \ldots)))))) \ldots \ldots \ldots \ldots$ \\
\hline & Non-native 2 & $((((((\ldots((\ldots \ldots)) \ldots \ldots)))))) \ldots(((\ldots \ldots))) \ldots$ \\
\hline & Non-native 3 & $((((((\ldots((\ldots \ldots \ldots)) \ldots \ldots)))))) \ldots \ldots \ldots \ldots$ \\
\hline & Non-native 4 & 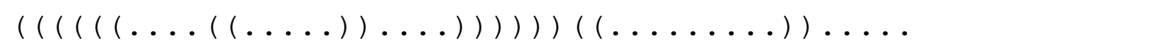 \\
\hline & Non-native 5 & $.((((((((\ldots \ldots)((((\ldots \ldots)))) \ldots))))) \ldots \ldots)).) \ldots$ \\
\hline \multirow[t]{7}{*}{$1 \mathrm{P} 5 \mathrm{O}$} & Sequence & $\begin{array}{l}\text { GGCUGUGAGGAACUACUGUCUUCACGCAGAAAGCGUCUAGCCAUGGCGUUAGUAUGAGUG } \\
\text { UCGUGCAGCCUCCAGCC }\end{array}$ \\
\hline & Native & 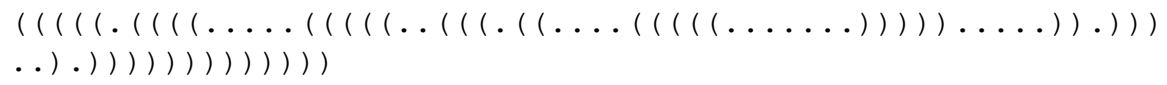 \\
\hline & Non-native 1 & 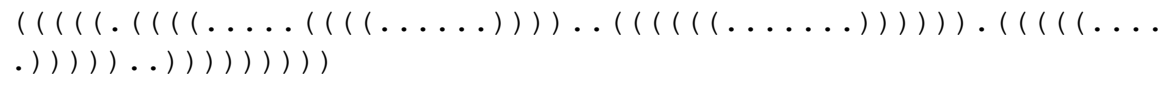 \\
\hline & Non-native 2 & 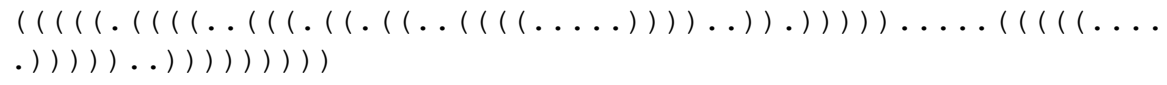 \\
\hline & Non-native 3 & $\begin{array}{l}(((((.((((\ldots \ldots \ldots \ldots \ldots)((((\ldots \ldots))((\ldots \ldots)) \ldots \ldots)))) \ldots(((((\ldots \ldots) \\
.))))) \ldots()))))))\end{array}$ \\
\hline & Non-native 4 & 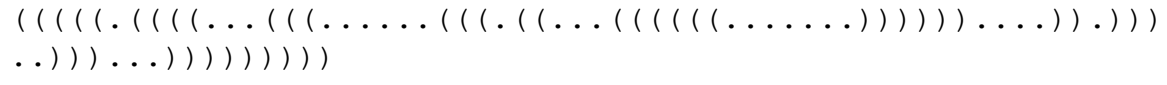 \\
\hline & Non-native 5 & 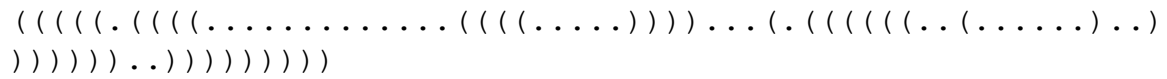 \\
\hline \multirow[t]{7}{*}{$3 \mathrm{MXH}$} & Sequence & $\begin{array}{l}\text { GUCACGCACAGGGCAAACCAUUCGAAAGAGUGGGACGCAAAGCCUCCGGCCUAAACCAUU } \\
\text { GCACUCCGGUAGGUAGCGGGGUUACCGAUGG }\end{array}$ \\
\hline & Native & 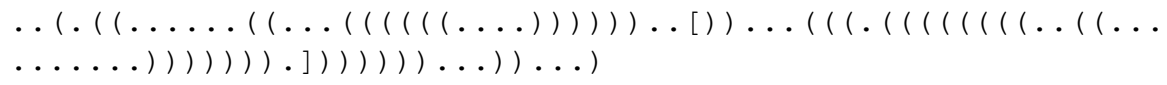 \\
\hline & Non-native 1 & 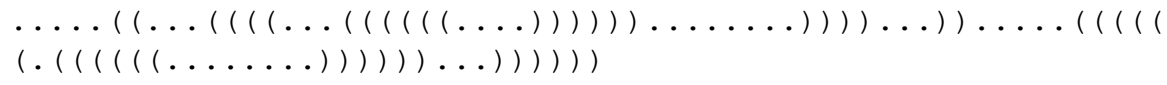 \\
\hline & Non-native 2 & 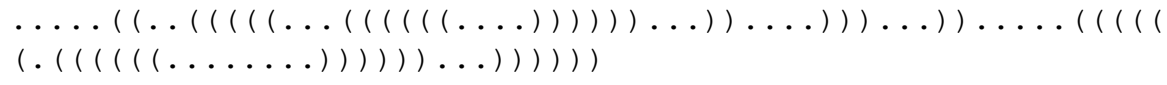 \\
\hline & Non-native 3 & 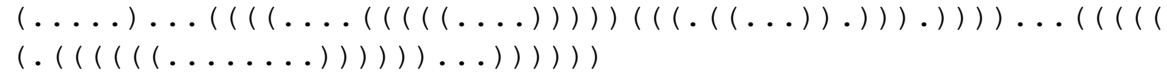 \\
\hline & Non-native 4 & $\begin{array}{l}\left.\left.\left.\left.((\ldots \ldots)) \cdot((((\ldots)(((((\ldots))))))(((\ldots((\ldots)) .))) .)))) \ldots\left(\left(\left(\left(\left({ }^{\prime}(\ldots)\right)\right)\right)\right)\right) \ldots(\ldots)\right)\right)\right)\right) \\
((((((\ldots \ldots \ldots))\end{array}$ \\
\hline & Non-native 5 & $\begin{array}{l}\ldots(((\ldots \ldots)((\ldots)((((((\ldots))))))) \ldots)) \ldots((((((((\ldots \ldots \ldots \ldots \ldots \\
\ldots \ldots())) .)))) .)))((\ldots \ldots)) \ldots \ldots\end{array}$ \\
\hline
\end{tabular}


Table B.8: Sequences, native 2D, and non-native 2D structures.

\begin{tabular}{|c|c|c|}
\hline PDB ID & Label & Structure \\
\hline \multirow[t]{8}{*}{$1 \mathrm{C} 2 \mathrm{X}$} & Sequence & UGCCUGGCGGCCGUAGCGCGGUGGUCCCACCUGACCCCAUGCCGAACUCAGAAGUGAAAC \\
\hline & & GCCGUAGCGCCGAUGGUAGUGUGGGGUCUCCCCAUGCGAGAGUAGGGAACUGCCAGGCAU \\
\hline & Native & 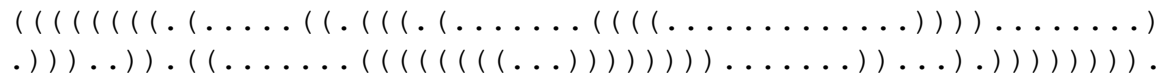 \\
\hline & Non-native 1 & 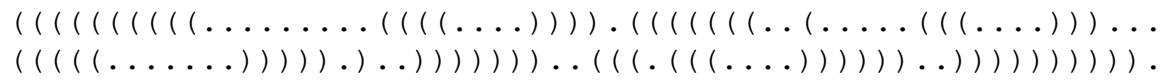 \\
\hline & Non-native 2 & 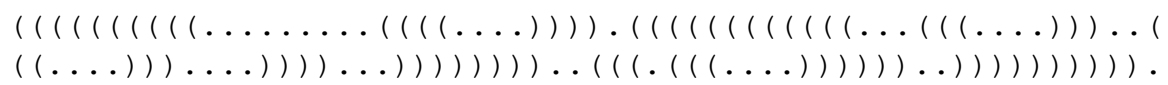 \\
\hline & Non-native 3 & 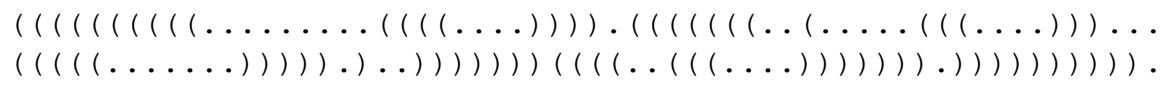 \\
\hline & Non-native 4 & 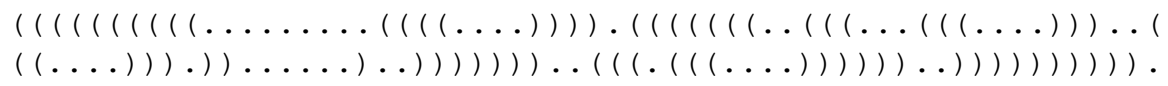 \\
\hline & Non-native 5 & 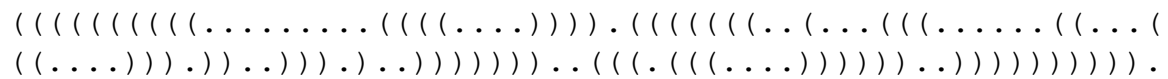 \\
\hline \multirow[t]{7}{*}{ 1GID } & Sequence & $\begin{array}{l}\text { GAAUUGCGGGAAAGGGGUCAACAGCCGUUCAGUACCAAGUCUCAGGGGAAACUUUGAGAU } \\
\text { GGCCUUGCAAAGGGUAUGGUAAUAAGCUGACGGACAUGGUCCUAACCACGCAGCCAAGUC } \\
\text { CUAAGUCAACAGAUCUUCUGUUGAUAUGGAUGCAGUUC }\end{array}$ \\
\hline & Native & 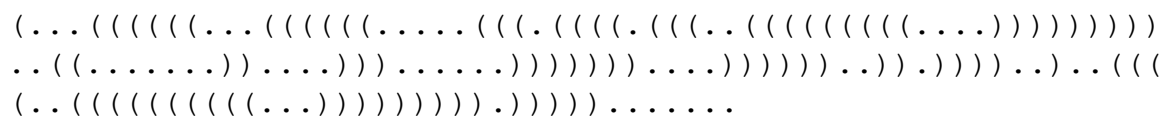 \\
\hline & Non-native 1 & 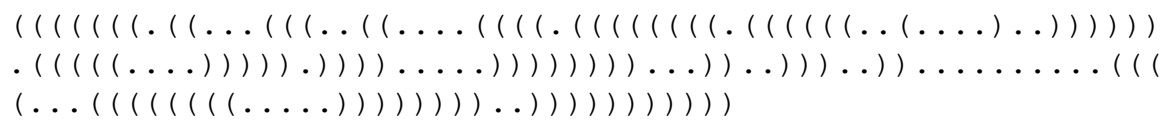 \\
\hline & Non-native 2 & 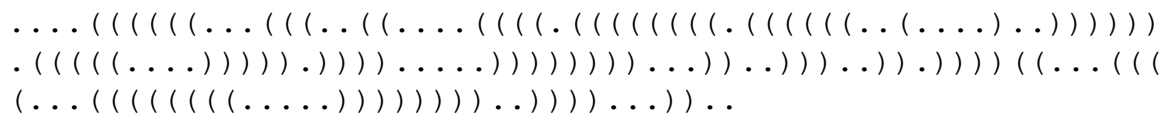 \\
\hline & Non-native 3 & 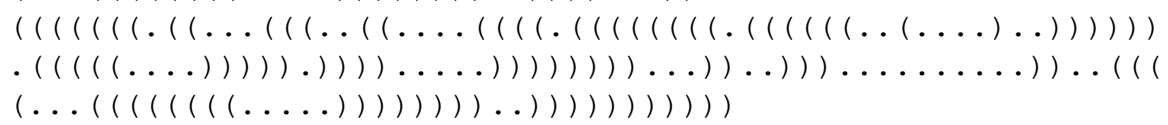 \\
\hline & Non-native 4 & 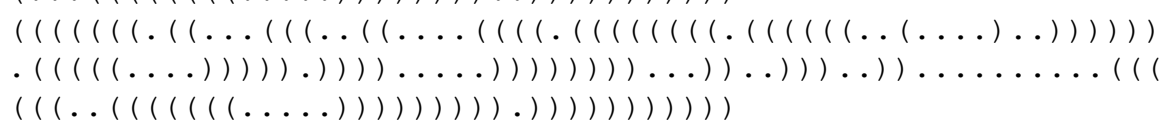 \\
\hline & Non-native 5 & 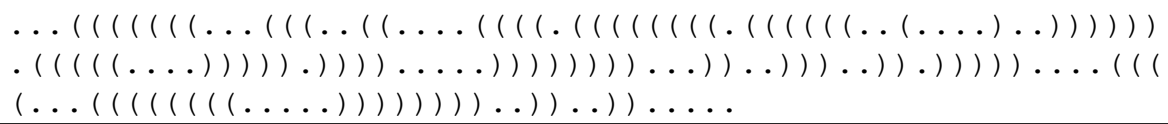 \\
\hline
\end{tabular}


Table B.9: Parameter optimization using the leave-one-out approach. The left out cases are labeled at the top. This table is continued on the next page, where averages and standard deviations for each parameter over all leave-one-out training runs are provided.

\begin{tabular}{|c|c|c|c|c|c|c|c|c|c|c|c|c|}
\hline param & 1AUD & $1 \mathrm{C} 2 \mathrm{X}$ & 1EHZ & 1GID & 1NBS & $1 \mathrm{P} 5 \mathrm{O}$ & 1VTQ & $1 \mathrm{Y} 26$ & 2GDI & 2K95 & 2L1V & $2 \mathrm{~L} 8 \mathrm{H}$ \\
\hline$A$ & 0.168 & 0.165 & 0.165 & 0.165 & 0.168 & 0.165 & 0.164 & 0.165 & 0.165 & 0.168 & 0.164 & 0.168 \\
\hline$B$ & -1.73 & -1.727 & -1.728 & -1.727 & -1.724 & -1.727 & -1.729 & -1.728 & -1.727 & -1.724 & -1.729 & -1.724 \\
\hline$C$ & 0.72 & 0.717 & 0.718 & 0.717 & 0.715 & 0.717 & 0.719 & 0.718 & 0.717 & 0.715 & 0.719 & 0.715 \\
\hline$D$ & 7.199 & 7.201 & 7.2 & 7.201 & 7.204 & 7.201 & 7.204 & 7.2 & 7.201 & 7.204 & 7.204 & 7.204 \\
\hline$A_{\mathrm{SAS}}^{0}$ & 19.033 & 19.051 & 19.043 & 19.051 & 19.066 & 19.051 & 19.037 & 19.043 & 19.051 & 19.066 & 19.037 & 19.066 \\
\hline$E_{2 D}$ & -0.448 & -0.442 & -0.445 & -0.442 & -0.437 & -0.442 & -0.446 & -0.445 & -0.442 & -0.437 & -0.446 & -0.437 \\
\hline$s_{0}$ & 0.229 & 0.231 & 0.23 & 0.231 & 0.233 & 0.231 & 0.23 & 0.23 & 0.231 & 0.233 & 0.23 & 0.233 \\
\hline$s_{1}$ & 0.579 & 0.574 & 0.575 & 0.574 & 0.57 & 0.574 & 0.577 & 0.575 & 0.574 & 0.57 & 0.577 & 0.57 \\
\hline$s_{2}$ & 0.004 & 0.007 & 0.006 & 0.007 & 0.01 & 0.007 & 0.004 & 0.006 & 0.007 & 0.01 & 0.004 & 0.01 \\
\hline$d_{0}$ & 0.041 & 0.044 & 0.043 & 0.044 & 0.047 & 0.044 & 0.041 & 0.043 & 0.044 & 0.047 & 0.041 & 0.047 \\
\hline$d_{1}$ & 0.909 & 0.913 & 0.911 & 0.913 & 0.917 & 0.913 & 0.908 & 0.911 & 0.913 & 0.917 & 0.908 & 0.917 \\
\hline$d_{2}$ & 0.028 & 0.031 & 0.03 & 0.031 & 0.034 & 0.031 & 0.028 & 0.03 & 0.031 & 0.034 & 0.028 & 0.034 \\
\hline$d_{3}$ & 0.042 & 0.045 & 0.044 & 0.045 & 0.049 & 0.045 & 0.043 & 0.044 & 0.045 & 0.049 & 0.043 & 0.049 \\
\hline$a_{0}$ & 0.058 & 0.059 & 0.058 & 0.059 & 0.06 & 0.059 & 0.058 & 0.058 & 0.059 & 0.06 & 0.058 & 0.06 \\
\hline$a_{1}$ & 0.441 & 0.434 & 0.437 & 0.434 & 0.428 & 0.434 & 0.439 & 0.437 & 0.434 & 0.428 & 0.439 & 0.428 \\
\hline$a_{2}$ & 0.099 & 0.104 & 0.101 & 0.104 & 0.108 & 0.104 & 0.1 & 0.101 & 0.104 & 0.108 & 0.1 & 0.108 \\
\hline$a_{3}$ & 0.101 & 0.105 & 0.103 & 0.105 & 0.11 & 0.105 & 0 . & 0.103 & 0.105 & 0.11 & 0.1 & 0.11 \\
\hline$w_{0}$ & 0.005 & 0.007 & 0.006 & 0.007 & 0.009 & 0.007 & 0.005 & 0.006 & 0.007 & 0.009 & 0.005 & 0.009 \\
\hline$w_{1}$ & 0.677 & 0.677 & 0.677 & 0.677 & 0.678 & 0.677 & 0.678 & 0.677 & 0.677 & 0.678 & 0.678 & 0.678 \\
\hline$w_{2}$ & 0.101 & 0.113 & 0.107 & 0.113 & 0.123 & 0.113 & 0.082 & 0.107 & 0.113 & 0.123 & 0.082 & 0.123 \\
\hline$w_{3}$ & 0.101 & 0.107 & 0.105 & 0.107 & 0.112 & 0.107 & 0.103 & 0.105 & 0.107 & 0.112 & 0.103 & 0.112 \\
\hline$E_{5^{\prime}}$ & -0.249 & -0.246 & -0.247 & -0.246 & -0.243 & -0.246 & -0.249 & -0.247 & -0.246 & -0.243 & -0.249 & -0.243 \\
\hline$E_{3^{\prime}}^{(A)}$ & -0.059 & -0.06 & -0.059 & -0.06 & -0.06 & -0.06 & -0.06 & -0.059 & -0.06 & -0.06 & -0.06 & -0.06 \\
\hline$E_{3^{\prime}}^{(U)}$ & -0.029 & -0.027 & -0.027 & -0.027 & -0.024 & -0.027 & -0.029 & -0.027 & -0.027 & -0.024 & -0.029 & -0.024 \\
\hline$E_{3^{\prime}}^{(G)}$ & -0.189 & -0.185 & -0.187 & -0.185 & -0.181 & -0.185 & -0.189 & -0.187 & -0.185 & -0.181 & -0.189 & -0.181 \\
\hline$E_{3^{\prime}}^{(C)}$ & -0.43 & -0.428 & -0.429 & -0.428 & -0.427 & -0.428 & -0.43 & -0.429 & -0.428 & -0.427 & -0.43 & -0.427 \\
\hline$c_{0}$ & 0.419 & 0.417 & 0.418 & 0.417 & 0.414 & 0.417 & 0.418 & 0.418 & 0.417 & 0.414 & 0.418 & 0.414 \\
\hline$c_{1}$ & 0.48 & 0.476 & 0.478 & 0.476 & 0.471 & 0.476 & 0.48 & 0.478 & 0.476 & 0.471 & 0.48 & 0.471 \\
\hline$c_{2}$ & 0.561 & 0.564 & 0.563 & 0.564 & 0.567 & 0.564 & 0.561 & 0.563 & 0.564 & 0.567 & 0.561 & 0.567 \\
\hline$c_{3}$ & 0.411 & 0.414 & 0.413 & 0.414 & 0.417 & 0.414 & 0.411 & 0.413 & 0.414 & 0.417 & 0.411 & 0.417 \\
\hline$F_{\text {term }}^{5^{\prime}}$ & 1.451 & 1.451 & 1.451 & 1.451 & 1.451 & 1.451 & 1.451 & 1.451 & 1.451 & 1.451 & 1.451 & 1.451 \\
\hline$F_{\text {term }}^{3^{\prime}}$ & 0.931 & 0.939 & 0.935 & 0.939 & 0.947 & 0.939 & 0.93 & 0.935 & 0.939 & 0.947 & 0.93 & 0.947 \\
\hline$F_{\text {sug }}^{\mathrm{C} 3 \mathrm{~N}}$ & 0.851 & 0.854 & 0.853 & 0.854 & 0.857 & 0.854 & 0.851 & 0.853 & 0.854 & 0.857 & 0.851 & 0.857 \\
\hline$F_{\text {sug }}^{\mathrm{C} 3 \mathrm{y}}$ & 1.221 & 1.146 & 1.183 & 1.146 & 1.07 & 1.146 & 1.222 & 1.183 & 1.146 & 1.07 & 1.222 & 1.07 \\
\hline$F_{\text {sug }}^{\mathrm{C} 4 \mathrm{~N}}$ & 1.061 & 1.057 & 1.059 & 1.057 & 1.054 & 1.057 & 1.06 & 1.059 & 1.057 & 1.054 & 1.06 & 1.054 \\
\hline$F_{\text {sug }}^{\mathrm{ClN}}$ & 0.711 & 0.714 & 0.713 & 0.714 & 0.717 & 0.714 & 0.711 & 0.713 & 0.714 & 0.717 & 0.711 & 0.717 \\
\hline$E_{\text {lig }}$ & -2.494 & -2.445 & -2.47 & -2.445 & -2.39 & -2.445 & -2.503 & -2.47 & -2.445 & -2.39 & -2.503 & -2.39 \\
\hline$\eta$ & 12.406 & 12.441 & 12.425 & 12.441 & 12.473 & 12.441 & 12.414 & 12.425 & 12.441 & 12.473 & 12.414 & 12.473 \\
\hline
\end{tabular}


Table B.10: Continuation of parameter optimization using the leave-one-out approach from previous page. The left out cases are labeled at the top.

\begin{tabular}{|c|c|c|c|c|c|c|c|}
\hline param & 3DIG & 3IWN & $3 \mathrm{MXH}$ & 3PDR & $4 \mathrm{KQY}$ & AVE & STDEV \\
\hline$A$ & 0.165 & 0.165 & 0.165 & 0.165 & 0.165 & 0.166 & 0.001 \\
\hline$B$ & -1.727 & -1.727 & -1.727 & -1.727 & -1.727 & -1.727 & 0.002 \\
\hline$C$ & 0.717 & 0.717 & 0.717 & 0.717 & 0.717 & 0.717 & 0.001 \\
\hline$D$ & 7.201 & 7.201 & 7.201 & 7.201 & 7.201 & 7.202 & 0.002 \\
\hline$A_{\mathrm{SAS}}^{0}$ & 19.051 & 19.051 & 19.051 & 19.051 & 19.051 & 19.05 & 0.01 \\
\hline$E_{2 D}$ & -0.442 & -0.442 & -0.442 & -0.442 & -0.442 & -0.442 & 0.003 \\
\hline$s_{0}$ & 0.231 & 0.231 & 0.231 & 0.231 & 0.231 & 0.231 & 0.001 \\
\hline$s_{1}$ & 0.574 & 0.574 & 0.574 & 0.574 & 0.574 & 0.574 & 0.002 \\
\hline$s_{2}$ & 0.007 & 0.007 & 0.007 & 0.007 & 0.007 & 0.007 & 0.002 \\
\hline$d_{0}$ & 0.044 & 0.044 & 0.044 & 0.044 & 0.044 & 0.044 & 0.002 \\
\hline$d_{1}$ & 0.913 & 0.913 & 0.913 & 0.913 & 0.913 & 0.913 & 0.003 \\
\hline$d_{2}$ & 0.031 & 0.031 & 0.031 & 0.031 & 0.031 & 0.031 & 0.002 \\
\hline$d_{3}$ & 0.045 & 0.045 & 0.045 & 0.045 & 0.045 & 0.045 & 0.002 \\
\hline$a_{0}$ & 0.059 & 0.059 & 0.059 & 0.059 & 0.059 & 0.059 & 0.001 \\
\hline$a_{1}$ & 0.434 & 0.434 & 0.434 & 0.434 & 0.434 & 0.434 & 0.004 \\
\hline$a_{2}$ & 0.104 & 0.104 & 0.104 & 0.104 & 0.104 & 0.104 & 0.003 \\
\hline$a_{3}$ & 0.105 & 0.105 & 0.105 & 0.105 & 0.105 & 0.105 & 0.003 \\
\hline$w_{0}$ & 0.007 & 0.007 & 0.007 & 0.007 & 0.007 & 0.007 & 0.001 \\
\hline$w_{1}$ & 0.677 & 0.677 & 0.677 & 0.677 & 0.677 & 0.677 & 0 \\
\hline$w_{2}$ & 0.113 & 0.113 & 0.113 & 0.113 & 0.113 & 0.11 & 0.012 \\
\hline$w_{3}$ & 0.107 & 0.107 & 0.107 & 0.107 & 0.107 & 0.107 & 0.003 \\
\hline$E_{5^{\prime}}$ & -0.246 & -0.246 & -0.246 & -0.246 & -0.246 & -0.246 & 0.002 \\
\hline$E_{3^{\prime}}^{(A)}$ & -0.06 & -0.06 & -0.06 & -0.06 & -0.06 & -0.06 & 0 \\
\hline$\stackrel{(U)}{E_{3^{\prime}}^{(U)}}$ & -0.027 & -0.027 & -0.027 & -0.027 & -0.027 & -0.027 & 0.002 \\
\hline$E_{3^{\prime}}^{(G)}$ & -0.185 & -0.185 & -0.185 & -0.185 & -0.185 & -0.185 & 0.003 \\
\hline$E_{3^{\prime}}^{(C)}$ & -0.428 & -0.428 & -0.428 & -0.428 & -0.428 & -0.428 & 0.001 \\
\hline$c_{0}$ & 0.417 & 0.417 & 0.417 & 0.417 & 0.417 & 0.417 & 0.001 \\
\hline$c_{1}$ & 0.476 & 0.476 & 0.476 & 0.476 & 0.476 & 0.476 & 0.003 \\
\hline$c_{2}$ & 0.564 & 0.564 & 0.564 & 0.564 & 0.564 & 0.564 & 0.002 \\
\hline$c_{3}$ & 0.414 & 0.414 & 0.414 & 0.414 & 0.414 & 0.414 & 0.002 \\
\hline$F_{\text {term }}^{5^{\prime}}$ & 1.451 & 1.451 & 1.451 & 1.451 & 1.451 & 1.451 & 0 \\
\hline$F_{\text {term }}^{3^{\prime}}$ & 0.939 & 0.939 & 0.939 & 0.939 & 0.939 & 0.938 & 0.005 \\
\hline$F_{\text {sug }}^{\mathrm{C} 3 \mathrm{~N}}$ & 0.854 & 0.854 & 0.854 & 0.854 & 0.854 & 0.854 & 0.002 \\
\hline$F_{\text {sug }}^{\mathrm{C} 3 \mathrm{X}}$ & 1.146 & 1.146 & 1.146 & 1.146 & 1.146 & 1.15 & 0.048 \\
\hline$F_{\text {sug }}^{\mathrm{CHN}}$ & 1.057 & 1.057 & 1.057 & 1.057 & 1.057 & 1.057 & 0.002 \\
\hline$F_{\text {sug }}^{\text {ClN }}$ & 0.714 & 0.714 & 0.714 & 0.714 & 0.714 & 0.714 & 0.002 \\
\hline$E_{\text {lig }}$ & -2.445 & -2.445 & -2.445 & -2.445 & -2.445 & -2.448 & 0.035 \\
\hline$\eta$ & 12.441 & 12.441 & 12.441 & 12.441 & 12.441 & 12.44 & 0.02 \\
\hline
\end{tabular}




\section{Table B.11: Sequences with SHAPE data used for reactivity bias calculation.}

\begin{tabular}{ll}
\hline$\#$ & Sequence \\
\hline 1 & GGCAGAUCUGAGCCUGGGAGCUCUCUGCC
\end{tabular}

GGCAGAGUCCUUCGGGACAUUGCACCUGCC

AGAGGUUCUAGUUAUACCCUCUAUAAAAAACUAA

AGAGGUUCUAGCUACACCCUCUAUAAAAAACUAA

GGGCUGUUUUUCUCGCUGACUUUCAGCCCCAAACAAAAAAUUGUCAGC

6 GGACGAUUGAGGAUAUUUAGACAUAGAGAAGGCAAUGGAGAGAGAGCUUUUU

7 GGGAUCUGUCACCCCAUUGAUCGCCUUCGGGCUGAUCUGGCUGGCUAGGCGGGUCCC

8 CGACACUAAUUGAUGGCCAUCCGAAUUCUUUUGGUCGCUGUCUGGCUGUCAGUAAGUAU

9 GCCAAAAAUCAAUAAGGAGACAAACAGAUGUGCGAACUCGAUGCUAGCAAAGGAGAAGAA

10 GGGAAAAAUCAAUAAGGAGACAACAAGAUGUGCGAACUCGAUGCUAGCAAAGGAGAAGAA

11 AAAAGCAAAAACCCCGAUAAUCUUCUUCAACUUUGGCGAGUACGAAAAGAUUACCGGGGCCCAC

12 GCAUUGGAGAUGGCAUUCCUCCAUUAACAAACCGCUGCGCCCGUAGCAGCUGAUGAUGCCUACAGA

13 UCGCACAUCUUGUUGUCUGAUUAUUGAUUUUUCCCGAAACCAUUUGAUCAUAUGACAAGAUGUGUAU

14 GGUGGCUUCGAGUAGAAGCCUUAUGUACCGGAAGGUGCGAAUCUUCCGAAGGAUCCGAGUAGGAUCCAA

15 GAAUUCUACCUAUCUGCUCUUGAAUUUGGGUAUUAAAGAGGAGAAAGGUACCAUGAGCAAAGGAGAAGAA

16 GAAUUCUACCAUUCACCUCUUGGAUUUGGGUAUUAAAGAGGAGAAAGGUACCAUGAGCAAAGGAGAAGAA

17 ACACCCAAAUUCAUGAGCAGAUUGGUAGUGGUGGUUAAUGAAAAUUAACUUACUACUACCUUUCUCUAGA

18 CGCUUCAUAUAAUCCUAAUGAUAUGGUUUGGGAGUUUCUACCAAGAGCCUUAAACUCUUGAUUAUGAAGUG

19 AAGGAAAACCCCCACUAUUUUUCCUCGAACUUGGCGGAACGCAGAAAAAUAAUGGGGGCCUCACAGAAUAC

20 GGGUCGUGACUGGCGAACAGGUGGGAAACCACCGGGGAGCGACCCCGGCAUCGAUAGCCGCCCCCCUGGGC

21 GGUCAGGCCGGCGAAAGUCGCCACAGUUUGGGGAAAGCUGUGCAGCCUGUAACCCCCCCACGAAAGUGC

21

22 GGACACUGAUGAUCGCGUGGAUAUGGCACGCAUUGAAUUUU

24 CCGUGAUAGUUUAAUGGUCAGAAUGGGCGCUUGUCGCGUGCCAGAUCGGGGUUCAAUUCCCCGUCGCGGAGCC

24 CCGUGAUAGUUUAAUGGUCAGAAUGGGCGCUUGUCGCGUGCCAGAUCGGGGUUCAAUUCCCCGUCGCGGAGCCA
25 GCGGAUUUAGCUCAGUUGGGAGAGCGCCAGACUGAAGAUCUGGAGGUCCUGUGUUCGAUCCACAGAAUUCGCACCA

25
26 GGGGAUUUAGGCUCAGUUGGGAGAGCGCCAGACUGAAGAUCUGGAGGUCCUGUGUUCGAUCCACAGAAUUCGCACCA

26 GGCUGUGAGGAACUACUGUCUUCACGCAGAAAGCGUCUAGCCAUGGCGUUAGUAUGAGUGUCGUGCAGCCUCCAGCC

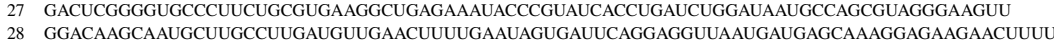

29 UUUAAACGGGUUUGCGGUGUAAGUGCAGCCCGUCUUACACCGUGCGGCACAGGCACUAGUACUGAUGUCGUCUACAGGGCUU

30 GGAAACGCAACGAGUAGUUGCGUUACGCAGCAGUCCUCCAGAGGACAUUUUUCGAGCAGGUAGAGAGGAUCCGAGUAGGAUCC

31 CACGCACAGGGCAAACCAUUCGAAAGAGUGGGACGCAAAGCCUCCGGCCUAAACGGCAUUGCACUCCGCCGUAGGUAGCGGGGUUACCGAUGG

32 GGAUCACGAGGGGGAGACCCCGGCAACCUGGGACGGACACCCAAGGUGCUCACACCGGAGACGGUGGAUCCGGCCCGAGAGGGCAACGAAGUCCGU

33 GGUGUCACGCACAGGGCAAACCAUUCGAAAGAGUGGGACGCAAAGCCUCCGGCCUAAACCAGAAGACAUGGUAGGUAGCGGGGUUACCGAUGGCAAAAUGCAUAC

34 AACAAAAUAAAAAGGAGUCGCUCACGCUUGGCGGUGUGAACGACAUCAUUCAAAGAAAAAAACACUGAGUUGUUUUUAUAAUCUUGUAUAUUUAGAUAUUAAACGA

35 GGGAAACUGCCUGAUGGAGGGGGAUAACUACUGGAAACGGUAGCUAAUACCGCAUAACGUCGCAAGACCAAAGAGGGGGACCUUCGGGCCUCUUGCCAUCGGAUGUGCCC

36 GUUCUUAUCAAGAGAAGCAGAGGGACUGGCCCGACGAAGCUUCAGCAACCGGUGUAAUGGCGAAAGCCAUGACCAAGGUGCUAAAUCCAGCAAGCUCGAACAGCUUGGAA GAUAAGAA

38 UGCCUGGCGGCCGUAGCGCGGUGGUCCCACCUGACCCCAUGCCGAACUCAGAAGUGAAACGCCGUAGCGCCGAUGGUAGUGUGGGGUCUCCCCAUGCGAGAGUAGGGAAC UGCCAGGCAU

39 GGAUCGCUGAACCCGAAAGGGGCGGGGGACCCAGAAAUGGGGCGAAUCUCUUCCGAAAGGAAGAGUAGGGUUACUCCUUCGACCCGAGCCCGUCAGCUAACCUCGCAAGC GUCCGAAGGAGAAUC

40 AACAAAAUAAAAAGGAGUCGCUCACGGAGGCCUUGGCGGGAGUCUGUGAACGACAUCAUUCAAAGAAAAAAACACUGAGUUGUUUUUAUAAUCUUGUAUAUUUAGAUAUU AAACGAGCCUCUACCUGCUUCGGCCGAUA

41 GGGAACCGAGUAGGUUCGCUUCAUAUAAUCCUAAUGAUAUGGUUUGGGAGUUUCUACCAAGAGCCUUAAACUCUUGAUUAUGAAGUCUGUCGCUUUAUCCGAAAUUUUAU AAAGAGAAGACUCAUGAAUGGGAUCCGAGUAGGAUCCU

42 GCCUAGCGAAGUCAUAAGCUAGGGCAGUCUUUAGAGGCUGACGGCAGGAAAAAAGCCUACGUCUUCGGAUAUGGCUGAGUAUCCUUGAAAGUGCCACAGUGACGAAGUCU CACUAGAAAUGGUGAGAGUGGAACGCGGUAAACCCCUCG

43 CUUCGUUAGGUGAGGCUCCUGUAUGGAGAUACGCUGCUGCCCAAAAAUGUCCAAAGACGCCAAUGGGUCAACAGAAAUCAUCGACAUAAGGUGAUUUUUAAUGCAGCUGG AUGCUUGUCCUAUGCCAUACAGUGCUAAAGCUCUACGAUUGAAG

44 UGCUGAAAUAUCUUCAUUUGAAUAAUAAAUUACUAUAUUAUUCAAUUAAUUAUUUAUAAUAAUAUAAUUUGAAAUAAAAAUAAUAUAGUUAAAAUAUUUAUUAUAAGAAG AaAaUUAGCAGUAAUUAAUAUAUAUAUAUAUAUAAAAUUAAUUAU

45 GAAUUGCGGGAAAGGGGUCAACAGCCGUUCAGUACCAAGUCUCAGGGGAAACUUUGAGAUGGCCUUGCAAAGGGUAUGGUAAUAAGCUGACGGACAUGGUCCUAACCACG CAGCCAAGUCCUAAGUCAACAGAUCUUCUGUUGAUAUGGAUGCAGUUC

46 GAUAUGAGGAGAGAUUUCAUUUUAAUGAAACACCGAAGAAGUAAAUCUUUCAGGUAAAAAGGACUCAUAUUGGACGAACCUCUGGAGAGCUUAUCUAAGAGAUAACACCG AAGGAGCAAAGCUAAUUUUAGCCUAAACUCUCAGGUAAAAGGACGGAG

47 GGCCUUCGGGCCAAUGUCACGCACAGGGCAAACCAUUCGAAAGAGUGGGACGCAAAGCCUCCGGCCUAAACCAGAAGACAUGGUAGGUAGCGGGGUUACCGAUGGCAAAA UGCAUACCCGAUCCGCUUCGGCGGAUCCAAAUCGGGCUUCGGUCCGGU 


\section{Table B.12: Continued table of sequences with SHAPE data used for reactivity bias cal- culation.}

\# $\quad$ Sequence

GGAACCGCGAGUAGCGGAAAUCCAGUAGGAACACUAUACUACUGGAUAAUCAAAGACAAAUCUGCCCGAAGGGCUUGAGAACAUCGAAACACGAUGCAGAGGUGGCAGCC UCCGGUGGGUUAAAACCCAACGUUCUCAACAAUAGUGAAAAGCGCGAGUAGCGCAAC

49 GGCCGACGGAGGCGCGCCCGAGAUGAGUAGGCUGUCCCAUCAGGGGAGGAAUCGGGGACGGCUGAAAGGCGAGGGCGCCGAAGGGUGCAGAGUUCCUCCCGCUCUGCAUC CCUGGGGGUAUGGGGAAUACCCAUACCACUGUCACGGAGGUCUCUCCGUGGAGAGCCGUCGGUC

50 CUUCGUUGGCCACAAUUAAAACAAACCAGAUCGUGGAGCUGCGCGAUCCCUUUGCAUAAAAACAUAUGGCUUUUGCUAUAAAAAUUAUGACUGCAAAACACCGGGCCAUU AAUAGCGUGCGGAGUGAUUUACGCGUUAUUGUUCUGCCGGGCGGACACGUGACGCGCGUGGCCAAU

51 GGAACACGGCAGGUGCUCCCGACCCUGCGGUCGGGAGUUAAAAGGGAAGCCGGUGCAAGUCCGGCACGGUCCCGCCACUGUGACGGGGAGUCGCCCCUCGGGAUGUGCCA CUGGCCCGAAGGCCGGGAAGGCGGAGGGGCGGCGAGGAUCCGGAGUCAGGAAACCUGCCUGCCGAAACG

52 GGCCUUCGGGCCAAAUGCCUGGCGGCCGUAGCGCGGUGGUCCCACCUGACCCCAUGCCGAACUCAGAAGUGAAACGCCGUAGCGCCGAUGGUAGUGUGGGGUCUCCCCAU GCGAGAGUAGGGAACUGCCAGGCAUCCGAUCCGCUUCGGCGGAUCCAAAUAAAUCGGGCUUCGGUCCGGU

53 GCGCUGUGUCGCAAUCUGCGAAGGGCGUCGUCGGCCCAAGCGGUAGUAAGCAGGGAACUCACCUCCAAUGAAACACAUUGUCGUAGCAGUUGACUACUGUUAUGUGAUUG GUAGAGGCUAAGUGACGGUAUUGGCGUAAGCCAAUACCGCAGCACAGCACAAGCCCGCUUGCGAGAUUACAGCGC

54 GGAACAGGUUGGGUUGGGAAGUAUCAUGGCUAAUCACCAUGAUGCAAUCGGGUUGAACACUUAAUUGGGUUAAAACGGUGGGGGACGAUCCCGUAACAUCCGUCCUAACG GCGACAGACUGCACGGCCCUGCCUCUUAGGUGUGUUCAAUGAACAGUCGUUCCGAAAGGAAGCAUCCGGUAUCCCAAGACAAUCAAACG

55 GGUCGUGCCUAGCGAAGUCAUAAGCUAGGGCAGUCUUUAGAGGCUGACGGCAGGAAAAAAGCCUACGUCUUCGGAUAUGGCUGAGUAUCCUUGAAAGUGCCACAGUGACG AAGUCUCACUAGAAAUGGUGAGAGUGGAACGCGGUAAACCCCUCGACCGAUCCGCUUCGGCGGAUCCCUUGAAAUCGGGCUUCGGUCCGGU

56 GCCGGUCCUGUGAGUUAAUAGGGAAUCCAGUGCGAAUCUGGAGCUGACGCGCAGCGGUAAGGAAAGGUGCGAUGAUUGCGUUAUGCGGACACUGCCAUUCGGUGGGAAGU CAUCAUCUCUUAGUAUCUUAGAUACCCCUCCAAGCCCGAAGACCUGCCGGCCAACGUCGCAUCUGGUUCUCAUCAUCGCGUAAUAUUGAUGAAACC

57 GGCAAUUCGAGUAGAAUUGACAGAGAGGAUAUGAGGAGAGAUUUCAUUUUAAUGAAACACCGAAGAAGUAAAUCUUUCAGGUAAAAAGGACUCAUAUUGGACGAACCUCU GGAGAGCUUAUCUAAGAGAUAACACCGAAGGAGCAAAGCUAAUUUUAGCCUAAACUCUCAGGUAAAAGGACGGAGAAAACACAAGUUCAGGAGUACUGAACC

58 CUCAUAUUUCGAUGUGCCUUGCGCCGGGAAACCACGCAAGGGAUGGUGUCAAAUUCGGCGAAACCUAAGCGCCCGCCCGGGCGUAUGGCAACGCCGAGCCAAGCUUCGGC GCCUGCGCCGAUGAAGGUGUAGAGACUAGACGGCACCCACCUAAGGCAAACGCUAUGGUGAAGGCAUAGUCCAGGGAGUGGCGAAAGUCACACAAACCGGAAUC

59 GGCCUUCGGGCCAAGAAUUGCGGGAAAGGGGUCAACAGCCGUUCAGUACCAAGUCUCAGGGGAAACUUUGAGAUGGCCUUGCAAAGGGUAUGGUAAUAAGCUGACGGACA UGGUCCUAACCACGCAGCCAAGUCCUAAGUCAACAGAUCUUCUGUUGAUAUGGAUGCAGUUCAAAACCCCGAUCCGCUUCGGCGGAUCCAAUAAAAUCGGGCUUCGGUCC GGU

60 GAAGCUGACCAGACAGUCGCCGCUUCGUCGUCGUCCUCUUCGGGGGAGACGGGCGGAGGGGAGGAAAGUCCGGGCUCCAUAGGGCAGGGUGCCAGGUAACGCCUGGGGGC GAAACCCACGACCAGUGCAACAGAGAGCAAACCGCCGAUGGCCCGCGCAAGCGGGAUCAGGUAAGGGUGAAAGGGUGCGGUAAGAGCGCACCGCGCGGCUGGUAACAGUC CGUGGCACGGUAAACUCCACCCGGAGCA

61 GCCGGGCGCGGUGGCGCGUGCCUGUAGUCCCAGCUACUCGGGAGGCUGAGGCUGGAGGAUCGCUUGAGUCCAGGAGUUCUGGGCUGUAGUGCGCUAUGCCGAUCGGGUGU CCGCACUAAGUUCGGCAUCAAUAUGGUGACCUCCCGGGAGCGGGGGACCACCAGGUUGCCUAAGGAGGGGUGAACCGGCCCAGGUCGGAAACGGAGCAGGUCAAAACUCC CGUGCUGAUCAGUAGUGGGAUCGCGCCUGUGAAUAGCCACUGCACUCCAGCCUGUGCAACAUAGCGAGACCCCGUCUCUUU

62 CCAUGAAUCACUCCCCUGUGAGGAACUACUGUCUUCACGCAGAAAGCGUCUAGCCAUGGCGUUAGUAUGAGUGUCGUGCAGCCUCCAGGACCCCCCCUCCCGGGAGAGCC AUAGUGGUCUGCGGAACCGGUGAGUACACCGGAAUUGCCAGGACGACCGGGUCCUUUCUUGGAUUAACCCGCUCAAUGCCUGGAGAUUUGGGCGUGCCCCCGCGAGACUG AUAGUGGUCUGCGGAACCGUGAGUACACCGGAAUUGCCAGGACGACCGGGUCCUUUCUUGGAUUAACCCGCUCAAUGCCUGGAGAUUUGGGCGUGCCCCCGCGAGACUG CCUCAA

63 AAGCGGGUGAAAAGCCCGCUCGCCGGAAGACCAAGGGUUCCUGUCCAACGUUAAUCGGGGCAGGGUGAGUCGACCCCUAAGGCGAGGCCGAAAGGCGUAGUCGAUGGGAA ACAGGUUAAUAUUCCUGUACUUGGUGUUACUGCGAAGGGGGGACGGAGAAGGCUAUGUUGGCCGGGCGACGGUUGUCCCGGUUUAAGCGUGUAGGCUGGUUUUCCAGGC AAUCCGGAAAAUCAAGGCUGAGGCGUGAUGACGAGGCACUACGGUGCUGAAGCAACAAAUGCCCUGCUUCCAGGAAAAGCCUCUAAGCAUCAGGUAACAUCAAAUCGUAC CCCAAACCGACACAGGUGGUCAGGUAGAGAAUACCAAGGCGC

64 GUGUGCCCGGCAUGGGUGCAGUCUAUAGGGUGAGAGUCCCGAACUGUGAAGGCAGAAGUAACAGUUAGCCUAACGCAAGGGUGUCCGUGGCGACAUGGAAUCUGAAGGAA GCGGACGGCAAACCUUCGGUCUGAGGAACACGAACUUCAUAUGAGGCUAGGUAUCAAUGGAUGAGUUUGCAUAACAAAACAAAGUCCUUUCUGCCAAAGUUGGUACAGAG UAAAUGAAGCAGAUUGAUGAAGGGAAAGACUGCAUUCUUACCCGGGGAGGUCUGGAAACAGAAGUCAGCAGAAGUCAUAGUACCCUGUUCGCAGGGGAAGGACGGAACAA GUAUGGCGUUCGCGCCUAAGCUUGAACCGCCGUAUACCGAACGGUACGUACGGUGGUGUGAGAGGAGUUCGCUCUACUCUAU

65 CUCUCUAAAUAGCAAUAUUUACCUUUGGAGGGAAAAGUUAUCAGGCAUGCACCUGGUAGCUAGUCUUUAAACCAAUAGAUUGCAUCGGUUUAAAAGGCAAGACCGUCAAA UUGCGGGAAAGGGGUCAACAGCCGUUCAGUACCAAGUCUCAGGGGAAACUUUGAGAUGGCCUUGCAAAGGGUAUGGUAAUAAGCUGACGGACAUGGUCCUACCACGCAG CCAAGUCCUAAGUCAACAGAUCUUCUGUUGAUAUGGAUGCAGUUCCAGACUAAAUGUCGGUCGGGGAAGAUGUAUUCUUCUCAUAAGAUAUAGUCGGACCUCUCCUUAAU GGGAGCUAGCGGAUGAAGUGAUGCAACACUGGAGCCGCUGGGAACUAAUUUGUAUGCGAAAGUAUAUUGAUUAGUUUUGGAGUACUCGUAAGGUA

66 GGUCAUUGCUAUUGGGGUCCGAUUUAGCCAUGCUAGUUGCACGAGUUCAUACUCGUGGCGAAAAGCUCAGUAACACGUGGCCAAACUACCCUACAGAGAACGAUAACCUC GGGAAACUGAGGCUAAUAGUUCAUACGGGAGUCAUGCUGGAAUGCCGACUCCCCGAAACGCUCAGGCGCUGUAGGAUGUGGCUGCGGCCGAUUAGGUAGACGGUGGGGUA ACGGCCCACCGUGCCGAUAAUCGGUACGGGUUGUGAGAGCAAGAGCCCGGAGACGGAAUCUGAGACAAGAUUCCGGGCCCUACGGGGCGCAGCAGGCGCGAAACCUUUAC ACUGCACGCAAGUGCGAUAAGGGGACCCCAAGUGCGAGGGCAUAUAGUCCUCGCUUUUCUCGACCGUAAGGCGGUCGAGGAAUAAGAGCUGGGCAAGACCGGUGCCAGCC GCCGCGGUAAUACCGGCAGCUCAAGUGAUGACC

67 UGACGGGGGCCCGCACAAGCGGUGGAGCAUGUGGUUUAAUUCGAUGCAACGCGAAGAACCUUACCUGGUCUUGACAUCCACGGAAGUUUUCAGAGAUGAGAAUGUGCCUU CGGGAACCGUGAGACAGGUGCUGCAUGGCUGUCGUCAGCUCGUGUUGUGAAAUGUUGGGUUAAGUCCCGCAACGAGCGCAACCCUUAUCCUUUGUUGCCAGCGGUCCGGC CGGGACUCAAAG AAGAGAAGCGACCUCGCGAGAGCAAGCGGACCUCAUAAAGUGCGUCGUAGUCCGGAUUGGAGUCUGCAACUCGACUCCAUGAAGUCGGAAUCGCUAGUAAUCGUGGAUCA GAAUGCCACGGUGAAUACGUUCCCGGGCCUUGUACACA 


\section{Table B.13: Continued table of sequences with SHAPE data used for reactivity bias cal- culation.}

\# Sequence

68 GGUCUCUCUGGUUAGACCAGAUCUGAGCCUGGGAGCUCUCUGGCUAACUAGGGAACCCACUGCUUAAGCCUCAAUAAAGCUUGCCUUGAGUGCUCAAAGUAGUGUGUGCC

CGUCUGUUGUGUGACUCUGGUAACUAGAGAUCCCUCAGACCCUUUUAGUCAGUGUGGAAAAUCUCUAGCAGUGGCGCCCGAACAGGGACUUGAAAGCGAAAGUAAAGCC GAGGAGAUCUCUCGACGCAGGACUCGGCUUGCUGAAGCGCGCACGGCAAGAGGCGAGGGGCGGCGACUGGUGAGUACGCCAAAAAUUUUGACUAGCGGAGGCUAGAAGGA GAGAGAUGGGUGCGAGAGCGUCGGUAUUAAGCGGGGGAGAAUUAGAUAAAUGGGAAAAAAUUCGGUUAAGGCCAGGGGGAAAGAAACAAUAUAAACUAAAACAUAUAGUA UGGGCAAGCAGGGAGCUAGAACGAUUCGCAGUUAAUCCUGGCCUUUUAGAGACAUCAGAA

69 GAUUGAACGCUGGCGGCAGGCCUAACACAUGCAAGUCGAACGGUAACAGGAAGAAGCUUGCUUCUUUGCUGACGAGUGGCGGACGGGUGAGUAAUGUCUGGGAAACUGCC UGAUGGAGGGGGAUAACUACUGGAAACGGUAGCUAAUACCGCAUAACGUCGCAAGACCAAAGAGGGGGACCUUCGGGCCUCUUGCCAUCGGAUGUGCCCAGAUGGGAUUA GCUAGUAGGUGGGGUAACGGCUCACCUAGGCGACGAUCCCUAGCUGGUCUGAGAGGAUGACCAGCCACACUGGAACUGAGACACGGUCCAGACUCCUACGGGAGGCAGCA GUGGGGAAUAUUGCACAAUGGGCGCAAGCCUGAUGCAGCCAUGCCGCGUGUAUGAAGAAGGCCUUCGGGUUGUAAAGUACUUUCAGCGGGGAGGAAGGGAGUAAAGUUAA GUGGGGAAUAUUGCACAAUGGGCGCAAGCCUGAUGCAGCCAUGCCGCGUGUAUGAAGAAGGCCUUCGGGUUGUAAAGUACUUUCAGCGGGGA

70 GGUCAGCGACUUAUAUUCUGUAGCAAGGUUAACCGAAUAGGGGAGCCGAAGGGAAACCGAGUCUUAACUGGGCGUUAAGUUGCAGGGUAUAGACCCGAAACCCGGUGAUC UAGCCAUGGGCAGGUUGAAGGUUGGGUAACACUAACUGGAGGACCGAACCGACUAAUGUUGAAAAAUUAGCGGAUGACUUGUGGCUGGGGGUGAAAGGCCAAUCAAACCG GGAGAUAGCUGGUUCUCCCCGAAAGCUAUUUAGGUAGCGCCUCGUGAAUUCAUCUCCGGGGGUAGAGCACUGUUUCGGCAAGGGGGUCAUCCCGACUUACCAACCCGAUG CAAACUGCGAAUACCGGAGAAUGUUAUCACGGGAGACACACGGCGGGUGCUAACGUCCGUCGUGAAGAGGGAAACAACCCAGACCGCCAGCUAAGGUCCCAAAGUCAUGG UUAAGUGGGAAACGAUGUGGGAAGGCCCAGACAGCCAGGAUGUUGGCUUAGAAGCAGCCAUCAUUUAAAGAAAGCGUAAUAGCUCACUGGUCGAGUCGGCCUGCGCGGAA GAUGUAACGGGGCUAAACCAUGCACCGAAGCUGCGGCAGCGACGCUUAUGCGUUGUUGGGUAGGGGAGCGUUCUGUAAGCCUGCGAAGGUGUGCUGUGAGGCAUGCUGGA GGUAUCAGAAGUGCGAAUGCUGACA

71 GUAAUCUUACCACUGUGUGUGUGCGUGUGUGUGUGUCGAGUCGUGUUGUCCGCACAUUUGAGUCGUGUUGUCCGCACAUUUUAUUUUUAUCUUUGUACAGUGUGUUAGAU UUCCCGAGGCAUGGCUUUCCAAGGUACCAGUAGGACUUUAACUCAACAGUCCUCAGCGGCUACGUCUGACGAUCUUCAAAAGAUAUUAUUUAGCCCUGAAGCCAUUAAGA AAAUGGCUACUGAGUGUGACCUAGGCCGGCAUCAUUGGAUGCGCGCUGAUAAUGCUAUUUCAGUCCGGCCCCUCGUUCCCGAAGUAACCCACGGUCGUAUUGCUUCCUUC UUUAAAUCUGGAUAUGAUGUUGGUGAAUUGUGCUCAAAAGGAUACAUGAGCGUCCCUCAGGUGUUGUGUGCUGUUACUCGAACGGUUUCCACCGAUGCUGAAGGGUCUUU GAGAAUUUACUUAGCUGAUCUAGGUGACAAGGAGUUAUCUCCUAUAGAUGGGCAAUGCGUUUCGUUACAUAACCAUGAUCUUCCCGCUUUGGUGUCUUUCCAACCGACGU AUGACUGUCCUAUGGAAACAGUUGGGAAUCGCAAGCGGUGUUUUGCUGUCGUUAUCGAAAGACAUGGUUACAUUGGGUAUACCGGUACCACAGCUAGCGUGUGUAGUAAU UGGCAAGCAAGGUUUUCUUCUAAGAAUAACAACUACACUCAUAUCGCAGCUGGGAAGACUCUAGUACUGCCUUUCAACAGAUUAGCUGAGCAAACAAAACCGUCAGCUGU UGCUCGCCUGUUGAAGUCGCAAUUGAACAACAUUGAAUCUUCGCAAUAUCUGUUAACGAAUGCGAAGAUUAAUCAGAAUGCGCGCAGUGAGUCCGAGGAAUUAAAUGUUG AGAGCCCUCCCGCCGCAAUCGGGAGUUCUUCCGCGUCCCGCUCCGAAGCCUUCAGACCGCAGGUGGUUAACGGUCUUUAGCACUUUGGUGCGUAUUAGUAUAUAAGUAUU UGUGAGUCUGUACAUAAUACUAUAUCUAUAGUGUCCUGUGUGAGUUGAUACAGUAGACAUCUGUGACGCGAUGCCGUGUUGAGAAGGGAACACAUCUGGUUUUAGUAAGC CUACAUCACAGUUUUGAGGUUCAAUUCCUCUUACUCCCUGUUGAGCCCCUUACUUUCUCAUGGAUGCUUCUCCGCGAGAUUGCGUUAUUGUCUACUGACUAUAUAGAGAG UGUAUGUGCUGUGUUUUCUCUUUUGUGUCGUAGAAUUGAGUUGAGUCAUGGACAAAUCUGAAUCAACCAGUGCUGGUCGCAACCGUCGACGUCGUCCGCGUCGUGGUUCC CGCUCCGCCCCCUCCUCCGCGGAUGCCAACUUUAGAGUCUUGUCGCAGCAGCUUUCGCGACUUAAUAAGACGUUGUCAGCUGGUCGUCCAACUAUUAACCACCCAACCUU UGUAGGGAGUGAGCGUUGUAAACCUGGAUACACGUUCACAUCUAUUACCCUAAAGCCACCAAAAAUAGACCGUGGGUCUUAUUAUGGUAAAAGGUUGUUAUUACCUGAUU CAGUCACAGAAUAUGAUAAGAAACUUGUUUCGCGCAUUCAAAUUCGAGUUAAUCCUUUGCCGAAAUUUGAUUCUACCGUGUGGGUGACAGUCCGUAAAGUUCCUGCCUCC UCGGACUUAUCCGUUGCCGCCAUCUCUGCUAUGUUUGCGGACGGAGCCUCACCGGUACUGGUUUAUCAGUAUGCCGCAUCUGGAGUCCAAGCUAACAACAAAUUGUUGUA UGAUCUUUCGGCGAUGCGCGCUGAUAUAGGCGACAUGAGAAAGUACGCCGUCCUCGUGUACUCAAAAGACGAUGCACUCGAGACGGACGAGCUAGUACUUCAUGUUGACG UCGAGCACCAACGUAUUCCCACAUCUGGGGUGCUCCCAGUAUAAUUCUGUGUUUUCCAGAACCCUCCCUCCGAUUCCUGUGGCGGGAGCUGAGUUGGCAGGUUUUCUAUA AACUGUCUGAAGUCACUAAACGUUUUACGGUGAACGGGUUGUCCAUCCAGCUUACGGCUAAAAUGGUCAGUCGUGGAGAAAUCUACGCCAGUAGACUUACAAGUCUCUGA GGCGCCUUUGAAACCAUCUCCUGGGUUUCUUCGGAAGGACUUCGGUCCGUGUACUUCUAGCACAAUGUGCUAGUUUCAGGGUAUGGGUGCCCCCCACUUUCGUGGGGGC CUCUAAAAGGAGACCA 


\section{B.3 Umbrella sampling to determine unreacted 1M7 sta- bility in the reactive site}

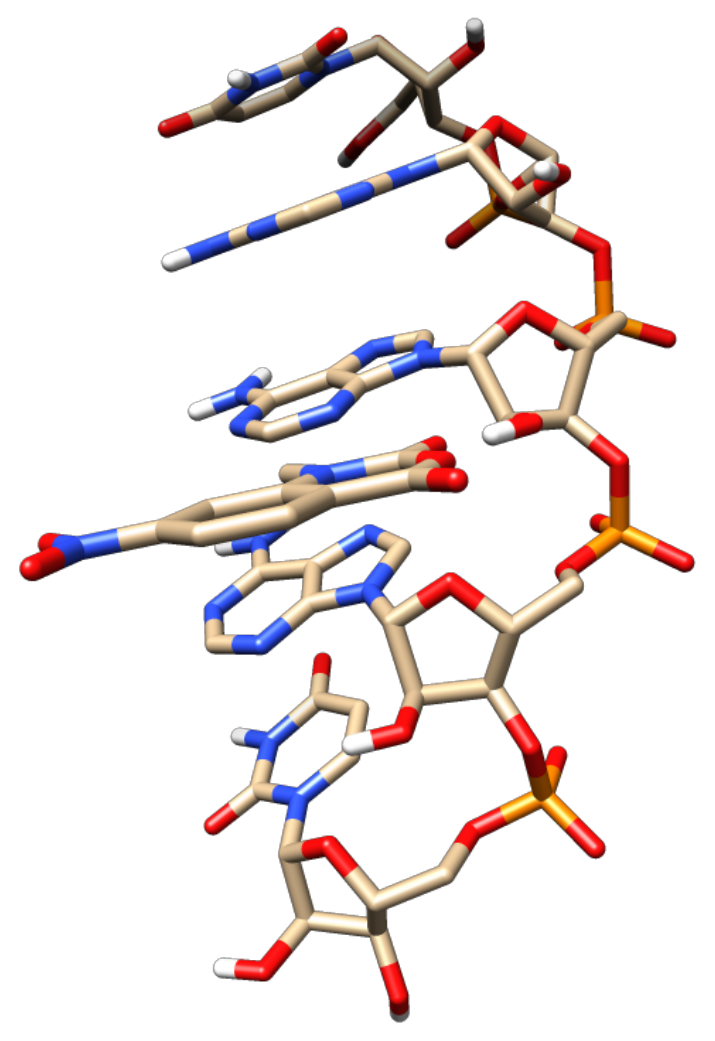

Figure B.1: 1M7 ligand in initial complex with the 5'UAAAU3' 5-mer. Since the ligand is initially positioned near the 2'-hydroxyl of the third nucleotide, it is between the third and fourth bases, which predisposes the SHAPE reagent to travel toward the 3' end of the 5-mer. 

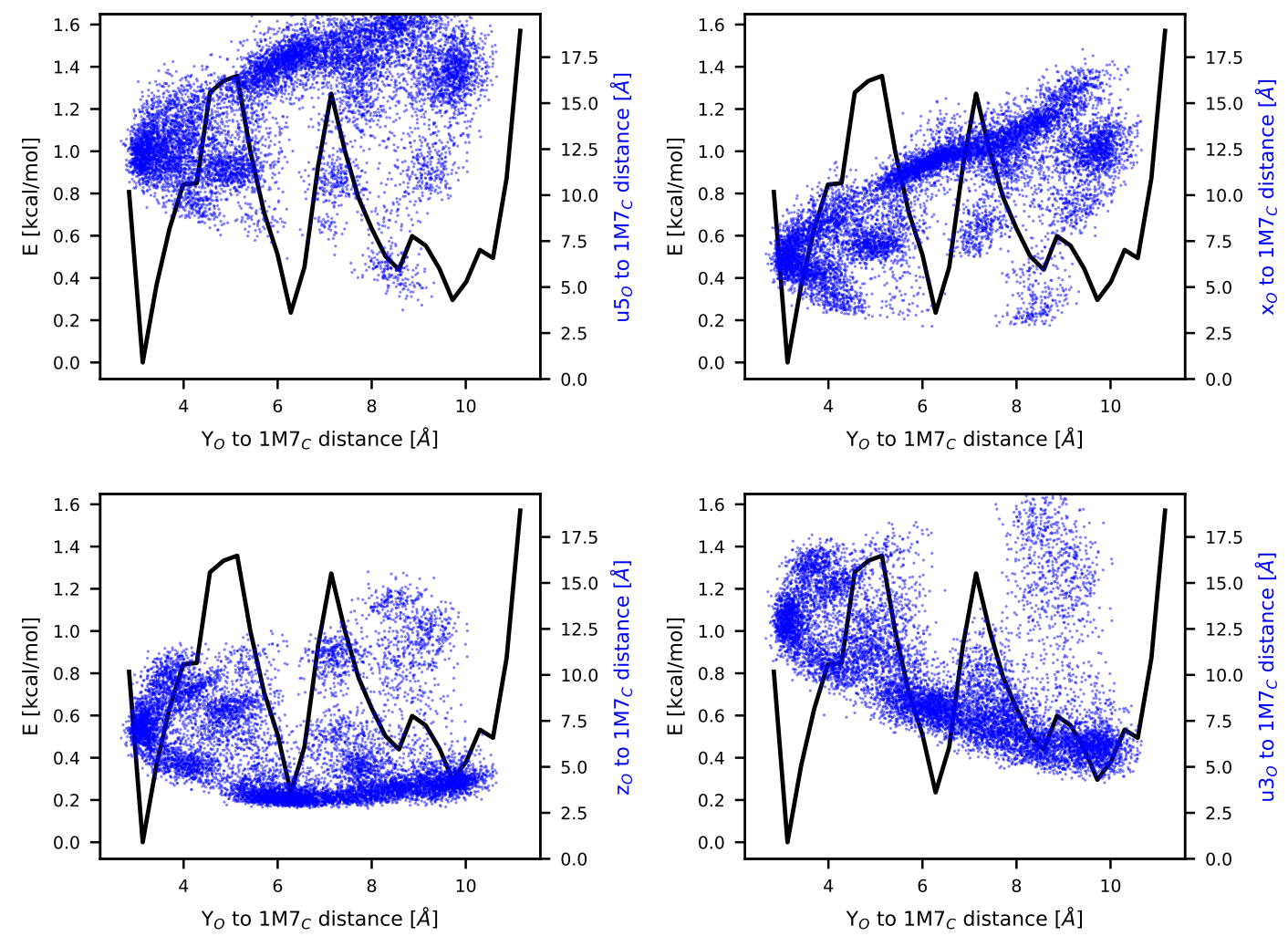

Figure B.2: PMF and various scatter density plots for normal umbrella sampling of the 5'UAAAU3' 5-mer. The x-axis for each panel is the distance from the 2'-oxygen of the 3A (Y in UXYZU 5-mer) nucleotide to reactive carbon of $1 \mathrm{M} 7$. The left $y$-axis for each panel is the PMF energy. The right y-axis for each panel is the distance from the 2'-oxygen of the 5' $\mathrm{U}, \mathrm{X}, \mathrm{Z}$, and 3' $\mathrm{U}$ nucleotides to the reactive carbon of $1 \mathrm{M} 7$. The $1 \mathrm{M} 7$ ligand tends to heavily travel toward the 3' end of the 5-mer and become trapped. The jagged PMF shows that transition state sampling in normal US is poor. 

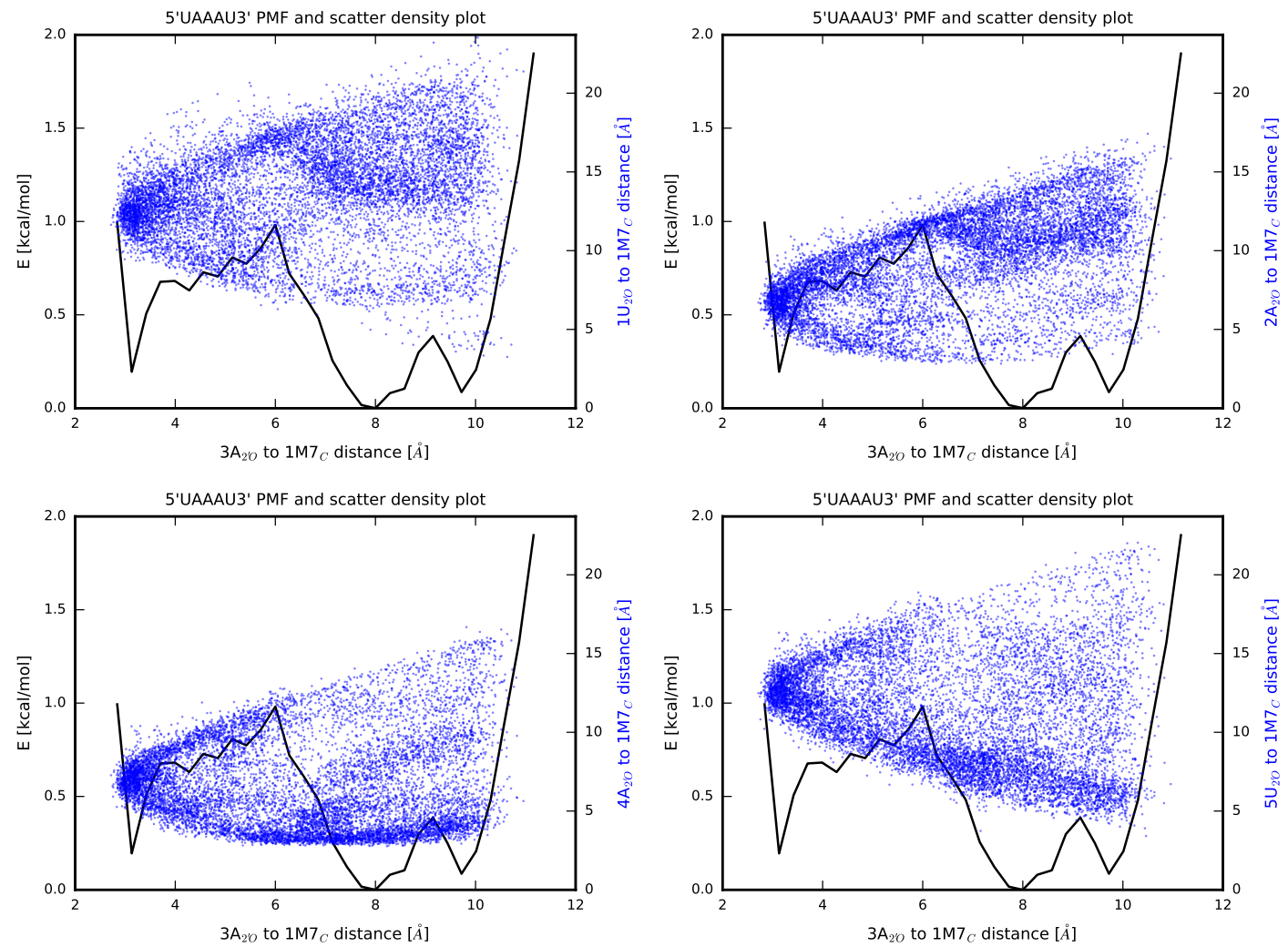

Figure B.3: PMF and various scatter density plots for REUS of the 5'UAAAU3' 5-mer. The xaxis for each panel is the distance from the 2'-oxygen of the 3A (Y in UXYZU 5-mer) nucleotide to reactive carbon of 1M7. The left $y$-axis for each panel is the PMF energy. The right $y$-axis for each panel is the distance from the 2'-oxygen of the 5' $\mathrm{U}, \mathrm{X}, \mathrm{Z}$, and 3' $\mathrm{U}$ nucleotides to the reactive carbon of $1 \mathrm{M} 7$. Although the ligand still tends to travel toward the 3 ' end, the sampling is much more uniform with the REUS implementation, and the transition states are lower energy than indicated by the normal US implementation, indicating improved transition state sampling. 


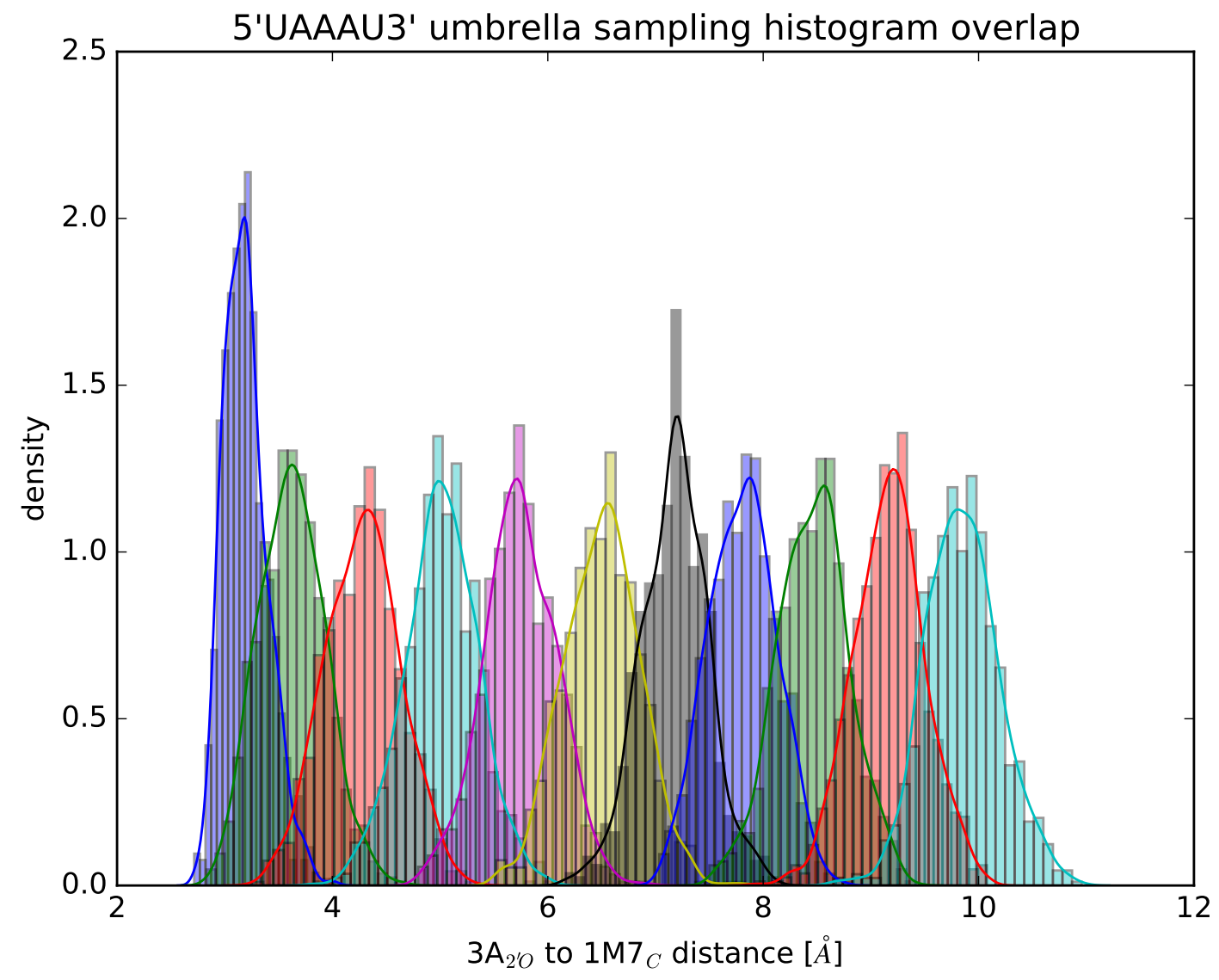

Figure B.4: Density histograms of the umbrellas for REUS with the 5'UAAAU3' sequence. Good overlap is observed with these biasing umbrellas, and the approximate normal distributions of the umbrellas indicates sufficient spring stiffness. 


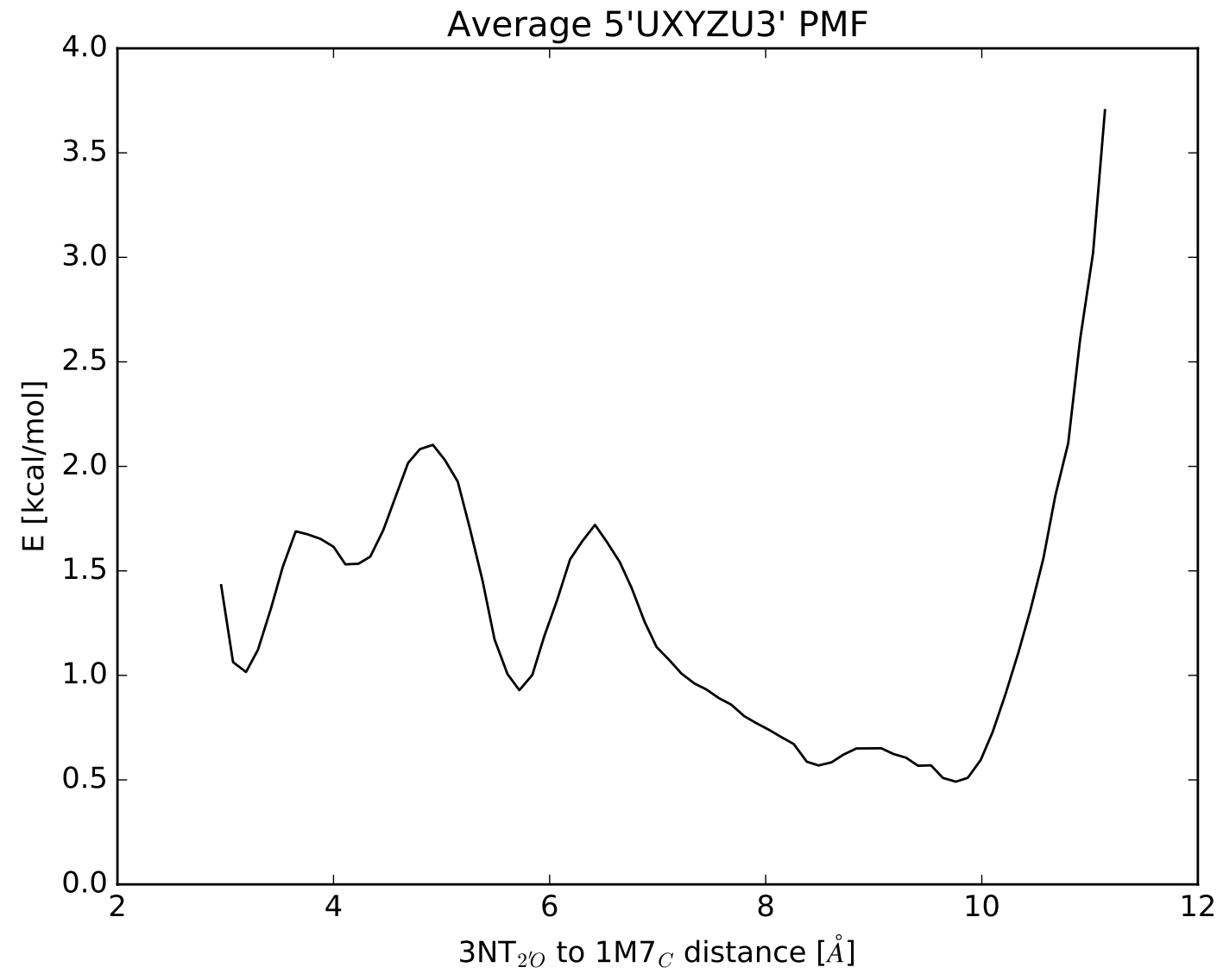

Figure B.5: PMF as a function of reaction coordinate distance from averaging all 5'UXYZU3' REUS runs. The average PMF provides a reference state and allows us to establish the relative binding stability of the 1M7 ligand in different sequence contexts. 


\section{B.4 Free energy calculations}

To calculate the relative free energies, we first calculate the average potential of mean force (PMF) from all 64 runs (see Fig. B.5). After calculating the average PMF, we determine the partition function for a portion of the PMF limited by reaction coordinates $a$ and $b$ as

$$
Z_{a \leftrightarrow b}=\sum_{i=a}^{b} e^{-\beta U_{i}}
$$

Then, we determine the free energy for a state by calculating

$$
G=-\beta^{-1} \ln Z
$$

which gives the free energy change between the reactive (rx) and alternative/unbound (ub) states for the 5'UXYZU3' 5-mer as

$$
\Delta G_{\mathrm{XYZ}}=G_{\mathrm{rX}}-G_{\mathrm{ub}}
$$

where for the reactive (unbound) state we calculate $Z$ for 2'-oxygen of nucleotide $\mathrm{Y}$ to the

reactive carbon of $1 \mathrm{M} 7$ distances $<5.0 \AA$ ( $\geq 5.0 \AA$ ). To estimate the relative stability, we find the free energy change relative to the average

$$
\Delta \Delta G_{\mathrm{XYZ}}=\Delta G_{\mathrm{XYZ}}-\Delta G_{\mathrm{ave}}
$$

Due to the paucity of data in the SHAPE sequences, we do not have the luxury of comparing these three-nucleotide, sequence-dependent relative free energy changes directly to the SHAPE data. To facilitate pairwise comparisons, we calculate the average free energy 
changes as

$$
\begin{aligned}
& <\Delta G_{\mathrm{XY}}>=\sum_{N=\mathrm{A}, \mathrm{U}, \mathrm{G}, \mathrm{C}} \Delta G_{\mathrm{XYN}} / 4 \\
& <\Delta G_{\mathrm{XZ}}>=\sum_{N=\mathrm{A}, \mathrm{U}, \mathrm{G}, \mathrm{C}} \Delta G_{\mathrm{XNZ}} / 4 \\
& <\Delta G_{\mathrm{YZ}}>=\sum_{N=\mathrm{A}, \mathrm{U}, \mathrm{G}, \mathrm{C}} \Delta G_{\mathrm{NYZ}} / 4
\end{aligned}
$$

We can similarly calculate the average relative free energy changes. To evaluate the strength of single nucleotide influence on the SHAPE reactivity, we can similarly calculate the average free energy changes as

$$
<\Delta G_{\mathrm{X}}>=\sum_{M, N=\mathrm{A}, \mathrm{U}, \mathrm{G}, \mathrm{C}} \Delta G_{\mathrm{XMN}} / 16
$$

However, we find that the pairwise calculations give us enough data points to draw a meaningful conclusion, while the single nucleotide calculations average too many effects and have too few data points to be very useful.

We can also perform this calculation for the $\mathrm{Y}$ and $\mathrm{Z}$ nucleotides. The single nucleotide influence on stability of the ligand occupying the reactive site can be seen in Table B.17. The pairwise nucleotide influence on the ligand-reactive site stability can be seen in Table B.16. The full set of energies extracted from the PMF can be seen in Table B.15. These were extracted using PMFs with 30 bins. 
Table B.14: Performance comparisons between new model and 3DSSR using Spearman rank correlation (SRC), Pearson correlation of the near-native ensemble $\left(\mathrm{PC}_{\mathrm{NN}}\right)$, and Pearson correlation of the top-ranked structure $\left(\mathrm{PC}_{\mathrm{T}}\right)$. Information provided below is from the left out test RNA in each training run, showing that the performance improvement applies to structures the model has not seen.

\begin{tabular}{llcccccc}
\hline PDB & \#nt & New SRC & 3DSSR SRC & New PC $_{\mathrm{NN}}$ & 3DSSR PC $_{\mathrm{NN}}$ & ${\text { New } \text { PC }_{\mathrm{T}}}^{\text {3DSSR PC }}$ \\
\hline 2L8H & 29 & 0.84 & 0.82 & 0.84 & 0.68 & 0.93 & 0.89 \\
1AUD & 30 & 0.82 & 0.69 & 0.89 & 0.76 & 0.95 & 0.86 \\
2L1V & 34 & 0.80 & 0.35 & 0.76 & 0.57 & 0.89 & 0.79 \\
2K95* & 47 & 0.75 & 0.59 & 0.56 & 0.36 & 0.64 & 0.49 \\
1Y26 & 71 & 0.74 & 0.62 & 0.90 & 0.76 & 0.94 & 0.86 \\
1VTQ & 74 & 0.65 & 0.43 & 0.76 & 0.51 & 0.84 & 0.59 \\
1EHZ & 76 & 0.72 & 0.72 & 0.77 & 0.54 & 0.84 & 0.63 \\
1P5O* & 77 & 0.88 & 0.88 & 0.66 & 0.56 & 0.71 & 0.64 \\
2GDI & 78 & 0.86 & 0.87 & 0.77 & 0.80 & 0.84 & 0.87 \\
3MXH* & 91 & 0.73 & 0.62 & 0.68 & 0.40 & 0.75 & 0.50 \\
3IWN & 93 & 0.89 & 0.89 & 0.82 & 0.60 & 0.89 & 0.68 \\
4KQY & 118 & 0.72 & 0.62 & 0.74 & 0.56 & 0.79 & 0.68 \\
1C2X* & 120 & 0.74 & 0.73 & 0.66 & 0.41 & 0.73 & 0.48 \\
1NBS & 149 & 0.72 & 0.77 & 0.65 & 0.55 & 0.72 & 0.64 \\
3PDR & 154 & 0.77 & 0.78 & 0.71 & 0.59 & 0.80 & 0.68 \\
1GID* & 158 & 0.67 & 0.70 & 0.62 & 0.38 & 0.68 & 0.47 \\
3DIG & 174 & 0.83 & 0.80 & 0.68 & 0.55 & 0.74 & 0.66 \\
\hline Average & 93 & 0.77 & 0.70 & 0.73 & 0.56 & 0.81 & 0.67 \\
\hline
\end{tabular}

${ }^{*}$ Structures used in new model that were not used to validate the previous 3DSSR model. 
Table B.15: Estimated stability of 1M7 in the reactive site for various XYZ combinations. These free energies from REUS PMF calculations form the basis for Tables B.17 and B.16

\begin{tabular}{|l|c|c|c|c|c|c|c|c|}
\hline XYZ & $\Delta G_{X Y Z}$ & $\Delta \Delta G_{X Y Z}$ & XYZ & $\Delta G_{X Y Z}$ & $\Delta \Delta G_{X Y Z}$ & XYZ & $\Delta G_{X Y Z}$ & $\Delta \Delta G_{X Y Z}$ \\
\hline GAC & 0.39 & -0.57 & CCU & 0.79 & -0.17 & CAC & 1.05 & 0.09 \\
\hline AAU & 0.4 & -0.56 & GUA & 0.8 & -0.16 & UUG & 1.06 & 0.09 \\
\hline GCU & 0.43 & -0.54 & AGA & 0.8 & -0.16 & UAC & 1.07 & 0.11 \\
\hline UCA & 0.44 & -0.53 & GUC & 0.86 & -0.1 & GUG & 1.12 & 0.15 \\
\hline GAG & 0.52 & -0.45 & UGU & 0.87 & -0.1 & ACG & 1.13 & 0.16 \\
\hline UAU & 0.54 & -0.43 & CAU & 0.88 & -0.08 & UUU & 1.15 & 0.18 \\
\hline GAU & 0.56 & -0.4 & CCG & 0.89 & -0.08 & CUG & 1.16 & 0.2 \\
\hline UAA & 0.57 & -0.4 & UAG & 0.89 & -0.07 & CCA & 1.17 & 0.21 \\
\hline UCC & 0.58 & -0.39 & GCG & 0.89 & -0.08 & UUC & 1.2 & 0.23 \\
\hline AUC & 0.6 & -0.37 & CGU & 0.9 & -0.06 & UCG & 1.21 & 0.24 \\
\hline UUA & 0.65 & -0.31 & AUU & 0.92 & -0.05 & CGA & 1.25 & 0.28 \\
\hline ACU & 0.66 & -0.31 & GCC & 0.92 & -0.05 & GGU & 1.25 & 0.28 \\
\hline ACC & 0.67 & -0.3 & GGA & 0.93 & -0.04 & AGG & 1.3 & 0.33 \\
\hline CAA & 0.69 & -0.28 & AUA & 0.94 & -0.03 & CGC & 1.31 & 0.34 \\
\hline CUC & 0.7 & -0.27 & CUU & 0.95 & -0.02 & UCU & 1.34 & 0.37 \\
\hline AUG & 0.71 & -0.25 & CUA & 0.96 & -0.01 & UGA & 1.46 & 0.49 \\
\hline AAC & 0.73 & -0.24 & CCC & 0.97 & 0.01 & CAG & 1.53 & 0.57 \\
\hline AGC & 0.74 & -0.23 & ACA & 0.99 & 0.02 & UGG & 1.56 & 0.59 \\
\hline AAA & 0.75 & -0.22 & GCA & 1.0 & 0.04 & CGG & 1.62 & 0.66 \\
\hline AGU & 0.78 & -0.18 & AAG & 1.01 & 0.04 & UGC & 1.68 & 0.71 \\
\hline GUU & 0.79 & -0.17 & GGC & 1.04 & 0.07 & GGG & 1.88 & 0.92 \\
\hline GAA & 0.79 & -0.18 & & & & & & \\
\hline
\end{tabular}


Table B.16: Stability in reactive site with respect to pairwise nucleotides from averaging over Table B.15.

\begin{tabular}{|c|c|c|c|c|c|c|c|c|}
\hline $\mathrm{XY}$ & $\left\langle\Delta G_{X Y}\right\rangle$ & $\left\langle\Delta \Delta G_{X Y}\right\rangle$ & $\mathrm{XZ}$ & $\left\langle\Delta G_{X Z}\right\rangle$ & $\left\langle\Delta \Delta G_{X Z}\right\rangle$ & $\mathrm{YZ}$ & $\left\langle\Delta G_{Y Z}\right\rangle$ & $\left\langle\Delta \Delta G_{Y Z}\right\rangle$ \\
\hline GA & 0.57 & -0.4 & $\mathrm{AC}$ & 0.68 & -0.28 & $\mathrm{AU}$ & 0.6 & -0.37 \\
\hline AA & 0.72 & -0.24 & $\mathrm{AU}$ & 0.69 & -0.27 & AA & 0.7 & -0.27 \\
\hline UA & 0.77 & -0.2 & GU & 0.76 & -0.21 & CC & 0.78 & -0.18 \\
\hline AU & 0.79 & -0.17 & UA & 0.78 & -0.18 & CU & 0.8 & -0.16 \\
\hline GC & 0.81 & -0.16 & GC & 0.8 & -0.16 & AC & 0.81 & -0.15 \\
\hline AC & 0.86 & -0.1 & AA & 0.87 & -0.09 & UA & 0.84 & -0.13 \\
\hline GU & 0.89 & -0.07 & CU & 0.88 & -0.08 & UC & 0.84 & -0.13 \\
\hline UC & 0.89 & -0.07 & GA & 0.88 & -0.08 & CA & 0.9 & -0.06 \\
\hline AG & 0.91 & -0.06 & UU & 0.97 & 0.01 & UU & 0.95 & -0.01 \\
\hline CU & 0.94 & -0.02 & CA & 1.01 & 0.05 & GU & 0.95 & -0.01 \\
\hline CC & 0.96 & -0.01 & CC & 1.01 & 0.04 & AG & 0.99 & 0.02 \\
\hline UU & 1.01 & 0.05 & AG & 1.04 & 0.07 & UG & 1.01 & 0.05 \\
\hline CA & 1.04 & 0.07 & GG & 1.1 & 0.14 & CG & 1.03 & 0.06 \\
\hline CG & 1.27 & 0.3 & UC & 1.13 & 0.17 & GA & 1.11 & 0.14 \\
\hline GG & 1.27 & 0.31 & UG & 1.18 & 0.22 & GC & 1.19 & 0.22 \\
\hline UG & 1.39 & 0.43 & CG & 1.3 & 0.34 & GG & 1.59 & 0.63 \\
\hline
\end{tabular}

Table B.17: Stability in reactive site from single nucleotide averaging over Table B.15.

\begin{tabular}{|c|c|c|c|c|c|c|c|c|}
\hline $\mathrm{X}$ & $\left\langle\Delta G_{X}\right\rangle$ & $\left\langle\Delta \Delta G_{X}\right\rangle$ & $\mathrm{Y}$ & $\left\langle\Delta G_{Y}\right\rangle$ & $\left\langle\Delta \Delta G_{Y}\right\rangle$ & $\mathrm{Z}$ & $\left\langle\Delta G_{Z}\right\rangle$ & $\left\langle\Delta \Delta G_{Z}\right\rangle$ \\
\hline $\mathrm{A}$ & 0.82 & -0.15 & $\mathrm{~A}$ & 0.77 & -0.19 & $\mathrm{~A}$ & 0.89 & -0.08 \\
\hline $\mathrm{C}$ & 1.05 & 0.09 & $\mathrm{C}$ & 0.88 & -0.09 & $\mathrm{C}$ & 0.91 & -0.06 \\
\hline $\mathrm{U}$ & 1.02 & 0.05 & $\mathrm{U}$ & 0.91 & -0.05 & $\mathrm{U}$ & 0.83 & -0.14 \\
\hline $\mathrm{G}$ & 0.89 & -0.08 & $\mathrm{G}$ & 1.21 & 0.24 & $\mathrm{G}$ & 1.16 & 0.19 \\
\hline
\end{tabular}




\section{Appendix C}

Appendix to Deciphering nucleotide modification-induced structure and stability changes 


\section{C.1 Thermal denaturation data for RNA duplexes}

Table C.1: Thermal denaturation data for base pairing of $\mathrm{m}^{6} \mathrm{~A}$ compared with A from previous work [175]. Good agreement between two-state fits and van't Hoff plots indicates the duplexes exhibit two-state behavior.

\begin{tabular}{|c|c|c|c|c|c|}
\hline Duplex & Abbrev. & $\begin{array}{c}\Delta G_{37}^{o} \text { fit } \\
(\mathrm{kcal} / \mathrm{mol})^{a}\end{array}$ & $\begin{array}{c}\Delta G_{37}^{o}{ }^{\mathrm{vH}} \\
(\mathrm{kcal} / \mathrm{mol})^{b}\end{array}$ & $\begin{array}{c}\Delta G_{37}^{o}{ }^{\text {avg }} \\
(\mathrm{kcal} / \mathrm{mol})^{c}\end{array}$ & $\begin{array}{l}\Delta \Delta G_{37}^{o}{ }^{\text {avg }} \\
(\mathrm{kcal} / \mathrm{mol})^{d}\end{array}$ \\
\hline $\begin{array}{l}\text { 5'-UACG A CUG } \\
\text { AUGC U GAC-3, }\end{array}$ & WT & $-10.7 \pm 0.1$ & $-10.9 \pm 0.2$ & $-10.8 \pm 0.2$ & 0 \\
\hline $\begin{array}{l}\text { 5'-UACG A CUG } \\
\text { AUGC A GAC-3' }\end{array}$ & A-A & $-6.1 \pm 0.1$ & $-6.0 \pm 0.2$ & $-6.1 \pm 0.2$ & $4.7 \pm 0.3$ \\
\hline $\begin{array}{l}\text { '-UACG A CUG } \\
\text { AUGC G GAC-3' }\end{array}$ & A-G & $-7.3 \pm 0.1$ & $-6.9 \pm 0.2$ & $-7.1 \pm 0.2$ & $3.7 \pm 0.3$ \\
\hline $\begin{array}{l}\text { 5'-UACG A CUG } \\
\text { AUGC C GAC-3' }\end{array}$ & A-C & $-8.0 \pm 0.1$ & $-8.5 \pm 0.1$ & $-8.3 \pm 0.1$ & $2.5 \pm 0.2$ \\
\hline $\begin{array}{l}\text { 5'-UACG }{ }^{\mathbf{m}} \text { ACUG } \\
\text { AUGC U GAC-3' }\end{array}$ & $\mathrm{m}^{6} \mathrm{~A}-\mathrm{U}$ & $-10.2 \pm 0.1$ & $-10.5 \pm 0.1$ & $-10.4 \pm 0.1$ & $0.4 \pm 0.2$ \\
\hline $\begin{array}{l}\text { 5'-UACG' } \mathbf{m}^{\mathbf{m}} \text { CUG } \\
\text { AUGC A GAC-3' }\end{array}$ & $\mathrm{m}^{6} \mathrm{~A}-\mathrm{A}$ & $-6.3 \pm 0.1$ & $-5.3 \pm 0.5$ & $-5.8 \pm 0.5$ & $5.0 \pm 0.5$ \\
\hline $\begin{array}{l}\text { 5'-UACG }{ }^{\mathbf{m}} \text { ACUG } \\
\text { AUGC G GAC-3' }\end{array}$ & $\mathrm{m}^{6} \mathrm{~A}-\mathrm{G}$ & $-6.8 \pm 0.1$ & $-7.0 \pm 0.2$ & $-6.9 \pm 0.2$ & $3.9 \pm 0.3$ \\
\hline $\begin{array}{l}\text { 5'-UACG }{ }^{\mathbf{m}} \text { ACUG } \\
\text { AUGC C GAC-3' }\end{array}$ & $\mathrm{m}^{6} \mathrm{~A}-\mathrm{C}$ & $-7.0 \pm 0.1$ & $-7.5 \pm 0.1$ & $-7.3 \pm 0.1$ & $3.5 \pm 0.2$ \\
\hline
\end{tabular}




\section{C.2 Running and quantitatively validating alchemical sim- ulations on duplex system}

\section{C.2.1 Setting up and running simulations}

Using tleap, the duplex and single-stranded structures were solvated in neutral, rectilinear water boxes with a $12.0 \AA$ A buffer, containing neutralizing magnesium with $0.2 \mathrm{M}$ sodium and chloride counterions. The hybrid system used in the Lennard-Jones (LJ) steps (one copy of both the WT and modified/mutated strands) was minimized for 2000 steps using the steepest descent algorithm, warmed to $300 \mathrm{~K}$ over $20 \mathrm{ps}$, and pressurized in the fully modified or mutated state $(\lambda=1)$ for 20 ps because the modified and mutated states generally have more atoms that can lead to clashes with the base pairing partner. All of the pre-production steps were performed with a $5.0 \mathrm{kcal} / \mathrm{mol}$ restraint on the heavy RNA atoms. After the equilibration, the hybrid system was decomposed and recombined to form the discharged (two copies of the WT strand) and charged systems (two copies of the modified strand). These files contain myriad atom and bond redundancies, so the Amber 18 parameter file editor (PARMED) [134] was used to remove redundant atoms and bonds and produce atom masks on only the transforming base for the remaining steps. Using the tiMerge function in PARMED, we merged many of the common atoms and bonds in the RNA molecules, reducing the soft core atom masks from over 380 atoms to the 30 or fewer base atoms, which allows us efficiently perform the remaining calculations and use GPUs for the production stage.

After creating the discharged, hybrid, and charged systems, each system was minimized, warmed, and pressurized at each alchemical control parameter $(\lambda)$ value $(\lambda=$ $0.0,0.1, \ldots, 1.0)$ using the Amber 18 CPU PMEMD implementation [134]. When $\lambda=0.0$, 
the system experiences the force field from the pure starting state, and when $\lambda=1.0$, the pure end state is fully realized. In between, the system experiences a mixed, soft core potential between the two states. For example, in the discharge step, the system will begin fully charged at $\lambda=0.0$, and the magnitude of the experienced electrical forces will decrease with increasing $\lambda$ until the evolving atoms have no charge at $\lambda=1.0$. For each $\lambda$, production simulations were carried out for $2.4 \mathrm{~ns}$ in an NVT ensemble under a Langevin thermostat with collision frequency of $2 \mathrm{ps}^{-1}$ using the Amber 18 GPU PMEMD implementation [213], and frames were written every 6 ps: 26,400 frames were extracted over a total of $158.4 \mathrm{~ns}$ of simulation time for each run. The simulations were repeated eight times, providing more robust and reproducible average results. Extending the simulations was attempted, but doubling the simulation time to $4.8 \mathrm{~ns}$ per $\lambda$ did not improve the agreement with experiment or reduce the calculated uncertainty (Table C.2). This aligns with previous findings, where longer simulations do not necessarily improve results due to bias from the initial velocities and drift away from states of interest [206, 214], but a larger ensemble of simulations might improve results. We note that although the single-stranded reference state simulations did not have time to completely sample the single-stranded conformational space, our use of an ensemble of short simulations for both duplex and single-stranded reference states helps form a basis of comparison. For instance, some mutations might enhance/diminish the stability of the helical single-stranded state by strengthening/weakening the single-stranded stacking interactions. Simulations starting with stronger single-stranded stacking states would sample fewer alternative conformations than their weaker counterparts and the basis for comparison between these simulation ensembles would be weakened. By limiting the single-stranded reference state simulations to short time periods and building ensembles of simulations, we ensured that the states we are 
comparing and using for reference across all the simulation ensembles are more uniform, which should improve our results.

\section{C.2.2 Validating duplex topology comparisons}

To validate that the ensembles duplex topologies are relatively uniform, we chose a structure from a run of the WT duplex as a reference, namely the last frame of the $5 \mathrm{~m}^{6} \mathrm{~A}$ decharge run $(\lambda=0.0)$. Then, we calculated the average heavy atom backbone RMSD for all the other available structures generated under pure potentials from this reference. The average WT RMSD is calculated by averaging the RMSD of all available WT conformations, including those in the decharge $5 \mathrm{~m}^{6} \mathrm{~A}$ run and similar conformations in decharge $(\lambda=0.0)$ steps for AA, AC, and AG mutations ( $\mathrm{RMSD}=1.3 \AA$ ). This provides a reference for how much the RMSD fluctuates in the WT case to illustrate how much more the modified/mutated cases deviate from an arbitrary WT duplex topology. Next, we calculated the average RMSD for each of the fully modified/mutated ensembles (RMSD = $1.6 \AA$ ). Since the average modified/mutated RMSD is not much different from the average WT RMSD, the induced topology changes are small, and our duplex state comparisons should be valid. To further establish that the duplex topology does not experience a large physical bulge for these modifications/mutations, we calculated the average distance between phosphate groups of the two residues. The average distances do not change by much: the average $\mathrm{WT}, 5 \mathrm{~m}^{6} \mathrm{~A}$ modified, purine-purine mutation, and $5 \mathrm{~m}^{6} \mathrm{~A}$ modified + purine-purine mutation distances were 19.6, 19.8, 19.9, and $20.5 \AA$, respectively, indicating a small amount of bulging occurs from modification/mutation, but not such a large amount that the duplex state comparison is invalidated. The trend of more bulging for larger perturbations is expected. 


\section{C.3 Enhancing hairpin sampling with TREMD}

Following previous work [215], the RNA hairpin was solvated in a TIP3P [88] truncated octahedral water box with a buffer of $10 \AA$ from the nearest part of the folded structure. To maintain a $1 \mathrm{M}$ sodium concentration and neutralize the system, sodium and chloride counterions were added. The Particle Mesh Ewald method (PMEMD) [216] in Amber 16 was used to treat long-range electrostatic interactions with non-bonded cutoffs of 10 $\AA$. The first minimization was carried out by holding the RNA fixed and minimizing the energy of ions and water molecules, using steepest descent minimization for 500 steps and conjugate gradient minimization for 500 steps. Next, the entire system energy was similarly minimized for 1500 steps of steepest descent and 1000 steps of conjugate gradient minimization. The system was equilibrated in an NVT ensemble (300 K) for 20 ps and an NPT ensemble $(1 \mathrm{~atm})$ for $100 \mathrm{ps.} \mathrm{Then,} \mathrm{the} \mathrm{replicas} \mathrm{were} \mathrm{created} \mathrm{and} \mathrm{equilibrated}$ at their respective starting temperatures for $100 \mathrm{ps}$. Finally, TREMD simulations were carried out using 24 replicas under a Langevin thermostat with collision frequency of 1 $\mathrm{ps}^{-1}$, consistent with a previous study [217]. The number of replicas was estimated using a temperature predictor [218], and temperatures were exponentially distributed from 265 to $365 \mathrm{~K}$. The temperature range was suggested by the predicted melting curve given by VfoldThermal [90] (see Fig. C.1). Exchanges were attempted every 50 steps (0.1 ps), and the exchange acceptance ratio ranged from 0.26 to 0.33 for the replicas, which is well above the recommended minimum exchange probability of 0.1 . The coordinates of all atoms were written to the Amber trajectory file every 5 ps, which yielded 60,000 snapshots of each replica over $300 \mathrm{~ns}$ and a total of $11.4 \mu$ s of simulation time per run.

To obtain orthogonal reaction coordinates, principal component (PC) analysis was carried out on the temperature-dependent trajectories for various simulation windows using 
CPPTRAJ [210]. Clustering was performed on the lowest temperature replica using the DBSCAN [219] algorithm for the first five PCs. Due to memory limitations, half of the frames were randomly chosen for the initial clustering, and the sieved frames were subsequently restored to their clusters for cluster ranking and calculation of cluster centroids. To find a suitable number of good clusters, the distance cutoff ( $5 \AA$ ) and minimum number of points to form a cluster (30) were found by locating extrema for the pseudo-F statistic (pSF) [220] and the Davies-Bouldin Index (DBI) [221] in the unmodified wild type (WT) RNA. Depending on the case, between 5,000 and 15,000 of the 50,000 frames corresponding to the 50 to $300 \mathrm{~ns}$ window were discarded as noise by the clustering algorithm. Representative frames corresponding to the centroid of each cluster were extracted for structural analysis. To compare the PMF of the modified RNA hairpins to the WT, the trajectories were projected along the PC eigenvectors found from the WT simulation by excluding atoms unique to the corresponding RNA hairpins from the CPPTRAJ mask. 


\section{C.4 Alchemical free energy estimation}

In thermodynamic integration (TI) calculations, the system transitions from one end state of interest to another through unphysical (alchemical) states according to a mixed potential $U(\lambda)$ in order to numerically estimate the integral [207]

$$
\Delta G=\int_{0}^{1}\left\langle\frac{\partial U(\lambda)}{\partial \lambda}\right\rangle_{\lambda} d \lambda
$$

where the alchemical control parameter $\lambda$ allows us to move from the starting state to the end state using the mixed potential

$$
U(\lambda)=(1-\lambda) U_{0}+\lambda U_{1}
$$

and $U_{0}$ and $U_{1}$ are the potentials for the pure starting and ending states, respectively. This change from one state to another is called an alchemical perturbation that is accomplished via alchemical simulations of the system. In order to form a thermodynamic cycle, we use the single stranded state to form a reference state. The relative free energy due to modification $\Delta \Delta G$ is determined by

$$
\Delta \Delta G=\Delta G_{\mathrm{A}}-\Delta G_{\mathrm{B}}=\Delta G_{\mathrm{C}}-\Delta G_{\mathrm{D}}
$$

Practically, we break the cycle into smaller steps, where for example,

$$
\Delta G_{\mathrm{A}}=\Delta G_{1}+\Delta G_{2}+\Delta G_{3}
$$


as described in Fig. 6.2 in the main text. Separating the electrostatic and Lennard-Jones (LJ) contributions to the free energy change allows accurate estimation of the free energy. If the electrostatic interactions are maintained while $\mathrm{LJ}$ interactions are removed, the charge of the atoms becomes more exposed, which can create large electrostatic forces and nonconvergent free energy differences [209]. 


\section{C.5 Estimated free energies from individual alchemical runs}

The systematic underestimation of uncertainty from estimators and the failure of longer simulations to improve results are shown in Table C.2. Doubling the simulation time does not improve the average uncertainties or agreement with experiment.

Table C.2: Free energies [kcal/mol] from eight runs. Estimated uncertainties from both trapezoidal thermodynamic integration (TI-T) and MBAR systematically underestimate the true uncertainty, calculated as the standard deviation of the values and shown in the "Ave." column.

\begin{tabular}{|c|c|c|c|c|c|c|c|c|c|}
\hline $2.4 \mathrm{~ns} / \lambda$ & Run 1 & Run 2 & Run 3 & Run 4 & Run 5 & Run 6 & Run 7 & Run 8 & Ave. \\
\hline ID & $\begin{array}{c}\text { TI-T } \\
\text { MBAR }\end{array}$ & $\begin{array}{c}\text { TI-T } \\
\text { MBAR }\end{array}$ & $\begin{array}{c}\text { TI-T } \\
\text { MBAR }\end{array}$ & $\begin{array}{c}\text { TI-T } \\
\text { MBAR }\end{array}$ & $\begin{array}{c}\text { TI-T } \\
\text { MBAR }\end{array}$ & $\begin{array}{c}\text { TI-T } \\
\text { MBAR }\end{array}$ & $\begin{array}{c}\text { TI-T } \\
\text { MBAR }\end{array}$ & $\begin{array}{c}\text { TI-T } \\
\text { MBAR }\end{array}$ & $\begin{array}{c}\text { TI-T } \\
\text { MBAR }\end{array}$ \\
\hline & $1.3 \pm 0.3$ & $-0.4 \pm 0.3$ & $1.5 \pm 0.3$ & $0.7 \pm 0.3$ & $0.3 \pm 0.3$ & $1.6 \pm 0.3$ & $-0.7 \pm 0.3$ & $1.6 \pm 0.3$ & $0.7 \pm 0.9$ \\
\hline $\mathrm{m}^{6} \mathrm{~A}-\mathrm{U}$ & $2 \pm 0.5$ & $0.5 \pm 0.5$ & $0.5 \pm 0.5$ & $1.8 \pm 0.5$ & $1.6 \pm 0.5$ & $1.3 \pm 0.5$ & $0.0 \pm 0.5$ & $1.2 \pm 0.6$ & $1.0 \pm 0.6$ \\
\hline & $3.6 \pm 0.3$ & $1.4 \pm 0.3$ & $0.2 \pm 0.3$ & $3.1 \pm 0.3$ & $2.2 \pm 0.3$ & $1.9 \pm 0.3$ & $3.1 \pm 0.3$ & $3.0 \pm 0.3$ & $2.3 \pm 1.1$ \\
\hline A-C & $5.1 \pm 0.5$ & $2.7 \pm 0.5$ & $1.4 \pm 0.5$ & $3.9 \pm 0.5$ & $1.0 \pm 0.5$ & $2.1 \pm 0.5$ & $3.3 \pm 0.5$ & $3.5 \pm 0.5$ & $2.9 \pm 1.4$ \\
\hline & $3.0 \pm 0.4$ & $1.2 \pm 0.4$ & $1.6 \pm 0.4$ & $1.3 \pm 0.4$ & $2.2 \pm 0.4$ & $3.3 \pm 0.4$ & $2.3 \pm 0.4$ & $2.7 \pm 0.4$ & $2.2 \pm 0.8$ \\
\hline $\mathrm{m}^{6} \mathrm{~A}-\mathrm{C}$ & $5.0 \pm 0.6$ & $3.8 \pm 0.6$ & $2.7 \pm 0.6$ & $1.7 \pm 0.6$ & $1.8 \pm 0.6$ & $4.2 \pm 0.6$ & $2.8 \pm 0.6$ & $3.6 \pm 0.6$ & $3.2 \pm 1.2$ \\
\hline & $3.1 \pm 0.3$ & $2.8 \pm 0.3$ & $1.9 \pm 0.3$ & $1.9 \pm 0.3$ & $3.2 \pm 0.3$ & $2.3 \pm 0.3$ & $2.5 \pm 0.3$ & $2.5 \pm 0.3$ & $2.5 \pm 0.5$ \\
\hline A-G & $3.9 \pm 0.6$ & $1.8 \pm 0.6$ & $3.2 \pm 0.5$ & $1.0 \pm 0.6$ & $3.2 \pm 0.5$ & $2.2 \pm 0.6$ & $2.4 \pm 0.6$ & $2.1 \pm 0.6$ & $2.5 \pm 0.9$ \\
\hline & $3.6 \pm 0.3$ & $2.7 \pm 0.3$ & $2.2 \pm 0.3$ & $3.1 \pm 0.3$ & $2.9 \pm 0.3$ & $2.5 \pm 0.3$ & $1.3 \pm 0.3$ & $2.6 \pm 0.3$ & $2.6 \pm 0.7$ \\
\hline $\mathrm{m}^{6} \mathrm{~A}-\mathrm{G}$ & $4.9 \pm 0.5$ & $1.6 \pm 0.5$ & $2.1 \pm 0.5$ & $4.0 \pm 0.5$ & $3.1 \pm 0.5$ & $1.5 \pm 0.5$ & $1.8 \pm 0.5$ & $2.8 \pm 0.6$ & $2.7 \pm 1.2$ \\
\hline & & & & & & $4.6 \pm 0.3$ & $3.5 \pm 0.3$ & $3.0 \pm 0.3$ & $3.4 \pm 0.9$ \\
\hline A-A & $3.0 \pm 0.5$ & $4.9 \pm 0.5$ & $6.3 \pm 0.5$ & $3.5 \pm 0.5$ & $1.9 \pm 0.5$ & $5.0 \pm 0.5$ & $5.0 \pm 0.6$ & $3.9 \pm 0.5$ & $4.2 \pm 1.4$ \\
\hline & $2.2 \pm 0.3$ & $2.6 \pm 0.3$ & $2.3 \pm 0.3$ & $2.0 \pm 0.3$ & $2.5 \pm 0.3$ & $3.5 \pm 0.3$ & $3.0 \pm 0.3$ & $3.0 \pm 0.3$ & $2.7 \pm 0.7$ \\
\hline $\mathrm{m}^{6} \mathrm{~A}-\mathrm{A}$ & $2.0 \pm 0.5$ & $3.0 \pm 0.5$ & $1.9 \pm 0.5$ & $2.8 \pm 0.5$ & $1.5 \pm 0.5$ & $4.6 \pm 0.5$ & $4.2 \pm 0.5$ & $4.6 \pm 0.5$ & $3.1 \pm 1.3$ \\
\hline $4.8 \mathrm{~ns} / \lambda$ & & & & & & & & & \\
\hline & $5 \pm 0.2$ & $0.6 \pm 0.2$ & $-0.3 \pm 0.2$ & $0.9 \pm 0.2$ & $0.5 \pm 0.2$ & $0.1 \pm 0.3$ & $1.2 \pm 0.3$ & $1.3 \pm 0.3$ & $0.7 \pm 0.6$ \\
\hline $\mathrm{m}^{6} \mathrm{~A}-\mathrm{U}$ & $1.5 \pm 0.4$ & $0.4 \pm 0.3$ & $-0.4 \pm 0.4$ & $1.8 \pm 0.4$ & $0.0 \pm 0.4$ & $1.1 \pm 0.4$ & $-0.6 \pm 0.4$ & $2.1 \pm 0.4$ & $0.7 \pm 1.0$ \\
\hline & $2.0 \pm 0.3$ & $2.1 \pm 0.3$ & $3.1 \pm 0.2$ & $2.2 \pm 0.3$ & $3.4 \pm 0.3$ & $1.4 \pm 0.3$ & $1.2 \pm 0.2$ & $3.3 \pm 0.3$ & $2.3 \pm 0.8$ \\
\hline$A-C$ & $1.7 \pm 0.4$ & $4.5 \pm 0.4$ & $4.4 \pm 0.3$ & $4.6 \pm 0.4$ & $5.2 \pm 0.4$ & $3.6 \pm 0.4$ & $1.3 \pm 0.4$ & $5.7 \pm 0.4$ & $3.9 \pm 1.6$ \\
\hline & $2.3 \pm 0.3$ & $3.2 \pm 0.3$ & $2.5 \pm 0.3$ & $2.2 \pm 0.3$ & $2.8 \pm 0.3$ & $2.0 \pm 0.3$ & $1.6 \pm 0.3$ & $1.7 \pm 0.3$ & $2.3 \pm 0.5$ \\
\hline $\mathrm{m}^{6} \mathrm{~A}-\mathrm{C}$ & $2.4 \pm 0.5$ & $5.1 \pm 0.5$ & $3.9 \pm 0.5$ & $4.9 \pm 0.5$ & $4.6 \pm 0.5$ & $4.4 \pm 0.5$ & $0.5 \pm 0.5$ & $2.7 \pm 0.5$ & $3.5 \pm 1.6$ \\
\hline & $2.9 \pm 0.3$ & $2.8 \pm 0.3$ & $3.0 \pm 0.3$ & $2.4 \pm 0.3$ & $3.2 \pm 0.3$ & $1.7 \pm 0.3$ & $2.7 \pm 0.3$ & $2.9 \pm 0.3$ & $2.7 \pm 0.4$ \\
\hline$A-G$ & $2.1 \pm 0.5$ & $3.6 \pm 0.4$ & $1.1 \pm 0.5$ & $1.5 \pm 0.5$ & $4.6 \pm 0.4$ & $2.3 \pm 0.4$ & $2.7 \pm 0.5$ & $2.5 \pm 0.5$ & $2.6 \pm 1.1$ \\
\hline & $3.4 \pm 0.3$ & $2.8 \pm 0.3$ & $1.5 \pm 0.3$ & $2.1 \pm 0.3$ & $3.1 \pm 0.2$ & $2.2 \pm 0.3$ & $2.7 \pm 0.3$ & $3.3 \pm 0.3$ & $2.6 \pm 0.7$ \\
\hline $\mathrm{m}^{6} \mathrm{~A}-\mathrm{G}$ & $5.3 \pm 0.4$ & $2.5 \pm 0.4$ & $1.3 \pm 0.4$ & $1.2 \pm 0.4$ & $3.6 \pm 0.4$ & $2.5 \pm 0.4$ & $1.6 \pm 0.4$ & $4.9 \pm 0.4$ & $2.9 \pm 1.6$ \\
\hline & $3.3 \pm 0.3$ & $4.5 \pm 0.3$ & $3.9 \pm 0.3$ & $2.8 \pm 0.3$ & $2.4 \pm 0.2$ & $3.6 \pm 0.3$ & $3.2 \pm 0.3$ & $2.5 \pm 0.3$ & $3.3 \pm 0.7$ \\
\hline A-A & $1.5 \pm 0.4$ & $5.2 \pm 0.4$ & $3.8 \pm 0.4$ & $4.9 \pm 0.4$ & $3.2 \pm 0.4$ & $4.6 \pm 0.4$ & $4.2 \pm 0.4$ & $4.4 \pm 0.4$ & $4.0 \pm 1.2$ \\
\hline & $3.3 \pm 0.2$ & $3.1 \pm 0.3$ & $2.7 \pm 0.3$ & $3.4 \pm 0.3$ & $1.5 \pm 0.2$ & $2.4 \pm 0.3$ & $1.6 \pm 0.3$ & $2.4 \pm 0.3$ & $2.5 \pm 0.7$ \\
\hline $\mathrm{m}^{6} \mathrm{~A}-\mathrm{A}$ & $2.2 \pm 0.4$ & $4.1 \pm 0.4$ & $4.3 \pm 0.4$ & $6.3 \pm 0.4$ & $3.3 \pm 0.4$ & $3.3 \pm 0.4$ & $1.5 \pm 0.4$ & $2.9 \pm 0.4$ & $3.5 \pm 1.4$ \\
\hline
\end{tabular}




\section{C.6 Predicted melting curve}

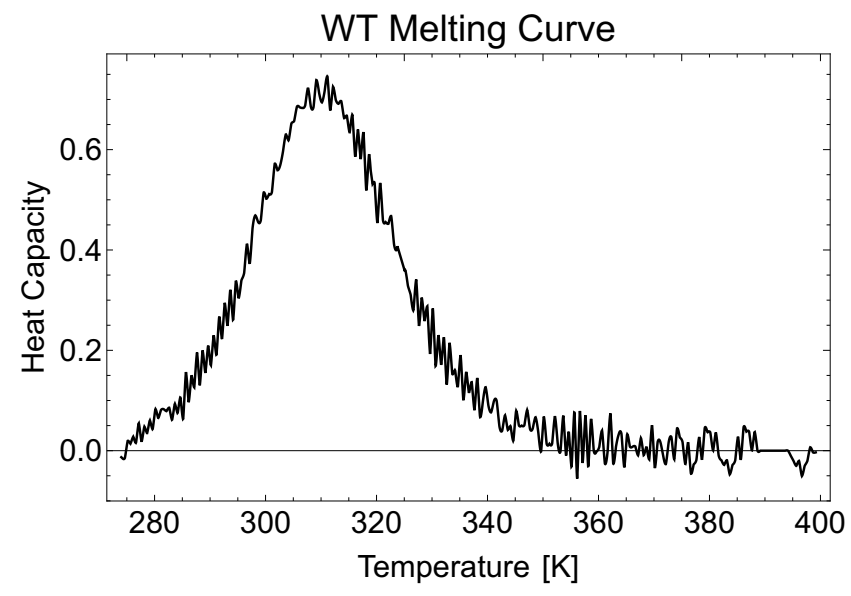

Figure C.1: The VfoldThermal-predicted melting curve for the wild type hairpin suggests an appropriate choice of temperature range [90]. The chosen range of 265 to $365 \mathrm{~K}$ was slightly broadened from the depicted optimal range to account for differences from modification or mutation. 


\section{C.7 Comparing estimators of relative free energy change}

Methods to estimate the free energy difference from alchemical simulations can be loosely categorized into two groups [209]: TI methods that provide different numerical estimates of Eq. C.1 to solve for the weights $W_{i}$ in

$$
\Delta G=\sum_{i=1}^{K} W_{i}\left\langle\frac{\partial U}{\partial \lambda}\right\rangle_{\lambda_{i}}
$$

and exponential averaging methods based on the Zwanzig relationship [222]

$$
\Delta G_{i j}=-\beta^{-1} \ln <e^{-\beta \Delta U_{i j}}>_{i}
$$

where the free energy difference $\Delta G_{i j}$ between for going from state $i$ to a perturbed state $j$ is estimated on the basis of exponential averaging over $i$, calculating the potential energy difference between adjacent states $\Delta U_{i j}$ in a simulated ensemble. The accuracy of methods based on the Zwanzig relationship depend on the overlap of the sampled energy distributions and not the smoothness of the integrand, while the accuracy of TI methods rely on the estimation of the average curvature $<\frac{\partial^{2} U}{\partial \lambda^{2}}>$ but not the overlap in the sampled energy distributions. Because of the differences in input information and the limitations of the different methods, we used the alchemical analysis python implementation (alchemical_analysis.py) of these different methods to identify sampling problems [209]. The TITrapezoid estimator is viewed as one of the preferred techniques in our analysis because of its flexibility and ease of use [208]. The TI-Cubic estimator was used to further validate the TI-Trapezoid estimations. Because methods based directly on the Zwanzig relationship depend on the direction of transformation, we also used BAR, which eliminates bias in $\Delta G$ 
estimation that are due to undersampling in the tail regions. BAR includes both forward $\Delta U_{i j}$ and reverse $\Delta U_{j i}$ potential energy differences in the analysis, which causes it to be a more robust estimator than direction-dependent estimation schemes. In BAR, the free energy change is computed from adjacent states to locally minimize the variance. Furthermore, the MBAR estimator—an extension of the BAR method—simultaneously optimizes all of the variances by sampling from the mixture distribution and yields the lowest variance estimate of the free energy change [209].

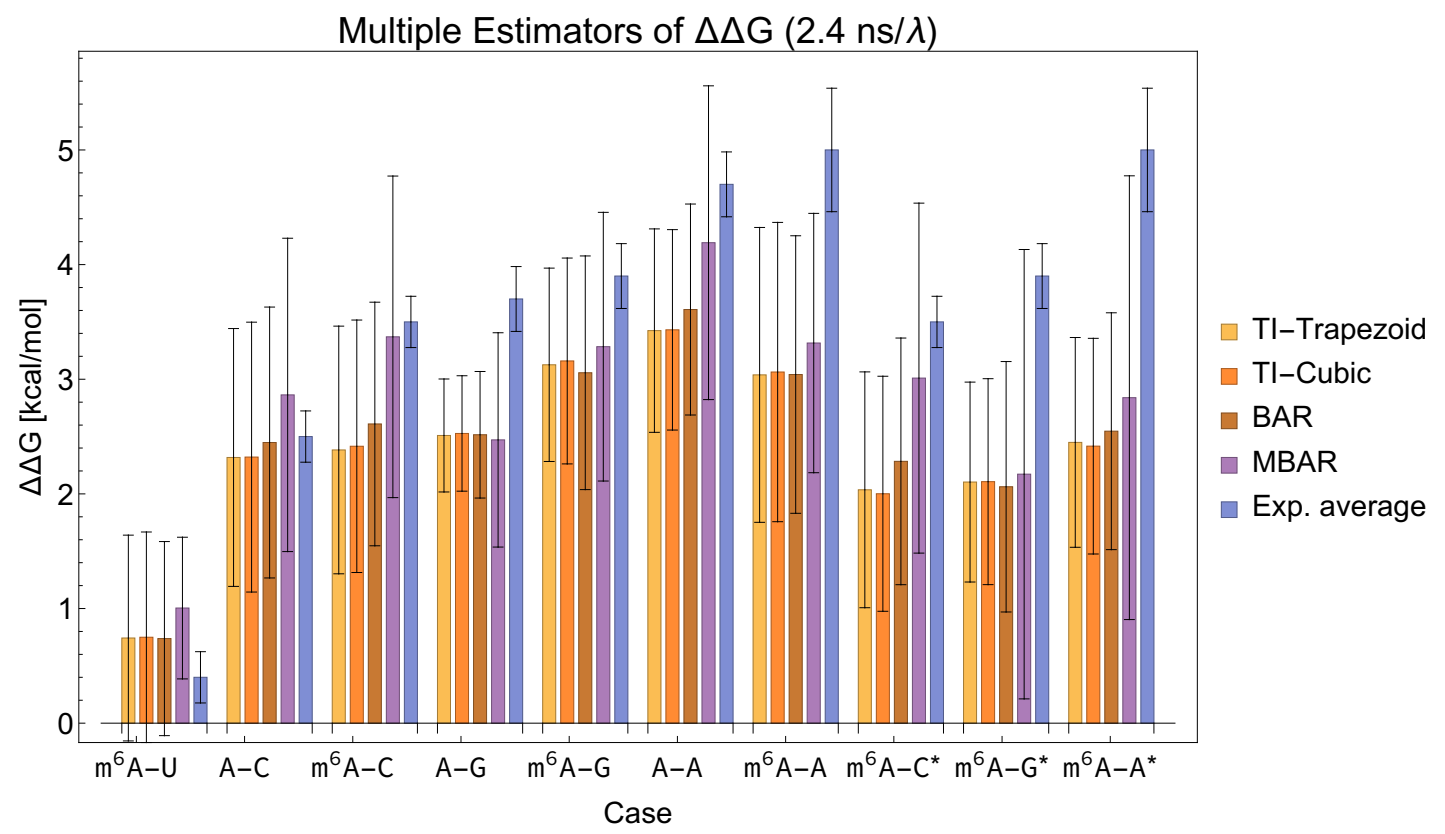

Figure C.2: The free energy of duplex formation due to modification extracted from alchemical simulations indicates favorable comparisons may be made between these simulations and thermal denaturation data, especially for relatively small perturbations of the duplex structure. Asterisks $(*)$ indicate the mutate-then-modify alchemical direction, where the nucleotide was mutated prior to being modified. Estimation methods are listed in the figure legend along with experimental data, labeled "Exp. average". 


\section{C.8 Calculating twice-perturbed free energies, propagat- ing uncertainty, and evaluating computational efficiency}

Here, we outline calculations of free energy from twice-perturbed states and explain the uncertainty estimation through quadrature. For instance, to go from the WT to a modified nucleotide $(x)$ and compare with experimental results, we must calculate the relative free energy change

$$
\Delta \Delta G^{\mathrm{WT} \rightarrow x}=\Delta G_{\mathrm{dup}}^{\mathrm{WT} \rightarrow x}-\Delta G_{\mathrm{ss}}^{\mathrm{WT} \rightarrow x}=\left(G_{\mathrm{dup}}^{x}-G_{\mathrm{dup}}^{\mathrm{WT}}\right)-\left(G_{\mathrm{ss}}^{x}-G_{\mathrm{ss}}^{\mathrm{WT}}\right)
$$

as before. Similarly, we can go to a mutated nucleotide $y$ using

$$
\Delta \Delta G^{\mathrm{WT} \rightarrow y}=\Delta G_{\mathrm{dup}}^{\mathrm{WT} \rightarrow y}-\Delta G_{\mathrm{ss}}^{\mathrm{WT} \rightarrow y}=\left(G_{\mathrm{dup}}^{y}-G_{\mathrm{dup}}^{\mathrm{WT}}\right)-\left(G_{\mathrm{ss}}^{y}-G_{\mathrm{ss}}^{\mathrm{WT}}\right)
$$

The $\Delta G$ values obtained by stepping through the thermodynamic cycle all have uncertainties associated with them $\delta g_{i}$. These uncertainties are propagated through quadrature to find the total uncertainty of the thermodynamic cycle

$$
\delta \delta g=\left(\sum_{i}^{N} \delta g_{i}^{2}\right)^{1 / 2}
$$

where $N$ is the number of alchemical steps in our thermodynamic cycle.

Twice-perturbed systems in our study undergo a modification and a mutation. In order to exhaustively sample the perturbative paths and accurately estimate the relative free energy we both modify-then-mutate and mutate-then-modify the system. Assuming the 
system is already modified, we can mutate the system using

$$
\Delta \Delta G^{\mathrm{x} \rightarrow x y}=\Delta G_{\mathrm{dup}}^{\mathrm{x} \rightarrow x y}-\Delta G_{\mathrm{ss}}^{\mathrm{x} \rightarrow x y}
$$

Alternatively, if the system is already mutated, we can modify it using

$$
\Delta \Delta G^{\mathrm{y} \rightarrow y x}=\Delta G_{\mathrm{dup}}^{\mathrm{y} \rightarrow y x}-\Delta G_{\mathrm{ss}}^{\mathrm{y} \rightarrow y x}
$$

Now, the relative free energy change of the modified-then-mutated system from the WT can be estimated as

$$
\Delta \Delta G^{\mathrm{WT} \rightarrow x y}=\Delta \Delta G^{\mathrm{WT} \rightarrow x}+\Delta \Delta G^{\mathrm{x} \rightarrow x y}
$$

Similarly, the relative free energy change of the mutated-then-modified system can be calculated as

$$
\Delta \Delta G^{\mathrm{WT} \rightarrow y x}=\Delta \Delta G^{\mathrm{WT} \rightarrow y}+\Delta \Delta G^{\mathrm{y} \rightarrow y x}
$$

Averaging these two results yields our best estimate of the twice-perturbed (tp) system

$$
\Delta \Delta G^{\mathrm{tp}}=\frac{\Delta \Delta G^{\mathrm{WT} \rightarrow x y}+\Delta \Delta G^{\mathrm{WT} \rightarrow y x}}{2}
$$

The uncertainties from these average through quadrature

$$
\delta \delta g^{\mathrm{tp}}=\frac{\left(\delta \delta g_{\mathrm{WT} \rightarrow x y}^{2}+\delta \delta g_{\mathrm{WT} \rightarrow y x}^{2}\right)^{1 / 2}}{2}
$$

and yield numerically estimated uncertainties on par with singly-perturbed systems. Analytically, the twice perturbed systems require 4 single perturbation steps ( 8 alchemical simulations) to yield 3 same-uncertainty, unique, relative free energies. This is due to the 
nature of the uncertainty calculation: assuming the uncertainty for a single perturbation is roughly constant $U[1]=a$, the uncertainties propagate as a function of the number of perturbations $n$

$$
U[n]=\frac{\sqrt{n\left(U^{2}[n-1]+a^{2}\right)}}{n}
$$

where $U[n]$ can be iteratively solved $(U[2]=a$, so the estimated singly-perturbed and twice-perturbed uncertainties are identical).

To evaluate the computational efficiency of applying multiple perturbations on our system, here we analytically solve for the efficiency. The exhaustive path sampling required to achieve an $n$-perturbed state yields

$$
\mathrm{FEC}=\sum_{i=1}^{n}\left(\begin{array}{l}
n \\
i
\end{array}\right)=2^{n}-1
$$

uniquely determined relative free energy changes (FEC) using

$$
\mathrm{SPS}=\sum_{i=0}^{n-1}\left(\begin{array}{l}
n \\
i
\end{array}\right)(n-i)=n \cdot 2^{n-1}
$$

single perturbation steps (SPS). While singly- and twice-perturbed simulations are relatively computationally efficient, this suggests that investigation of multiply-perturbed systems would be computationally inefficient, with an efficiency of

$$
\eta=\frac{\mathrm{FEC}}{\mathrm{SPS}}=\frac{2-2^{1-n}}{n}
$$

Although analyzing the uncertainty estimated from single runs of these systems provides insight into the efficiency of these types of calculations, the estimated uncertainty from a single run is much lower than the true uncertainty because these systems depend 
so strongly on initial conditions, and larger system perturbations will lead to larger true uncertainties. 


\section{C.9 Confirming convergence of hairpin TREMD PMF}

While absolute convergence of the PMF for a hairpin of this size would take microseconds per replica, the replicas reach equilibrium with each other fairly quickly. To be safe, we discard the first $50 \mathrm{~ns}$ of simulation time in most of our analysis. Fig. C.3 shows the PMF for various simulation windows, which confirms reasonable convergence was achieved in our simulations.
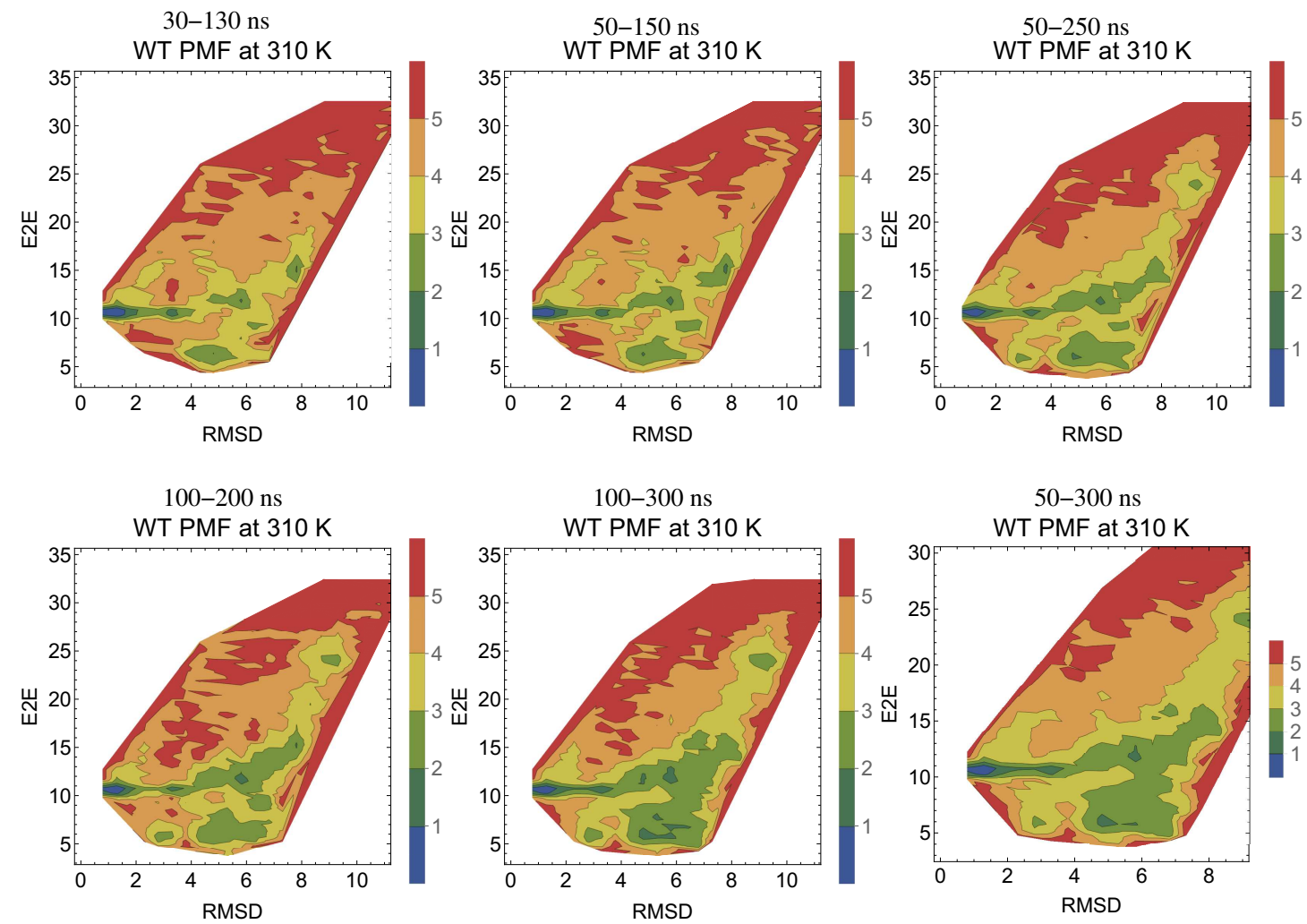

Figure C.3: Qualitative convergence of the wild type (WT) hairpin PMF is confirmed by viewing various simulation windows of increasing time. Most major changes to the PMF occur before 30 ns. To be safe, we used the 50 to $300 \mathrm{~ns}$ window in our structural ensemble analysis. 


\section{C.10 Calculating free energy changes from hairpin PMFs}

Experimentally, the free energy of duplex formation is reported with respect to the single stranded state; i.e., the free energy of the single stranded state is set to zero. The configurational partition function in a region of the PMF is calculated using

$$
Z_{a \leftrightarrow b}=\sum_{i=a}^{b} e^{-\beta U_{i}}
$$

where $Z_{a \leftrightarrow b}$ is the partition function within the boundaries of the reaction coordinates ( $a$ and $b), U_{i}$ is the value of the PMF, and $\beta=1 / k_{B} T$. From the partition function, we can calculate the free energy for a state as

$$
F=-\beta^{-1} \ln Z
$$

After calculating the free energy for the native hairpin $(\mathrm{NH})$ and random coil $(\mathrm{RC})$ conformations, the change in free energy is found using

$$
\Delta F=F_{\mathrm{NH}}-F_{\mathrm{RC}}
$$

After establishing the change in free energy from the $\mathrm{NH}$ to a RC conformation, we calculate the relative change in free energy due to modification or mutation as

$$
\Delta \Delta G=\Delta F_{\text {nonWT }}-\Delta F_{\mathrm{WT}},
$$

which resembles $\Delta \Delta G=\Delta G_{C}-\Delta G_{D}$ from Fig. 6.2 and Eq. 6.1 in the main text.

To establish boundaries for the $\mathrm{NH}$ and $\mathrm{RC}$ conformations in the calculation of the free 
energy change due to modification using the 2D RMSD/E2E PMFs, a free energy uncertainty cutoff of 0.065 (95\% confidence calculated using MBAR and assuming uncorrelated data) was used to determine the maximum RMSD boundary of the folded state. This resulted in defining the $\mathrm{NH}$ as having an E2E distance of 8.3 to $10.7 \AA$ for all cases and an RMSD of 0.0 to $1.4 \AA$ in all cases except three. Hairpins with noncanonical base pairs between $2 \mathrm{~A}$ and the ninth nucleotide (AA, $\mathrm{AC}$, and $\mathrm{AG}$ ) required $\mathrm{RMSD} \leq 1.8 \AA$ to meet the uncertainty cutoff. The portion of the PMF not contained in the NH boundaries are considered to be RC conformations, so the entire PMF is considered in the estimation of the free energy change. 


\section{C.11 Evaluating convergence and phase space overlap in alchemical simulations}

\begin{tabular}{c|c|c|c|c|c|c|c|c|c|c|c|c|}
$\lambda$ & 0 & 1 & 2 & 3 & 4 & 5 & 6 & 7 & 8 & 9 & 10 \\
\hline 0 & .62 & .12 & .16 & .04 & .04 & .01 & .01 & & & & \\
\hline 1 & .41 & .11 & .23 & .08 & .09 & .04 & .02 & .01 & .01 & & \\
\hline 2 & .19 & .07 & .24 & .12 & .17 & .09 & .06 & .03 & .01 & & \\
\hline 3 & .07 & .04 & .19 & .13 & .23 & .14 & .11 & .06 & .03 & & \\
\hline 4 & .03 & .02 & .14 & .11 & .24 & .18 & .15 & .09 & .04 & & \\
\hline 5 & .02 & .01 & .09 & .09 & .22 & .19 & .18 & .12 & .07 & .01 & \\
\hline 6 & .01 & .01 & .06 & .06 & .17 & .17 & .20 & .17 & .12 & .02 & .01 \\
\hline 7 & & & .03 & .03 & .10 & .12 & .18 & .20 & .22 & .06 & .04 \\
\hline 8 & & & .01 & .01 & .04 & .06 & .11 & .19 & .34 & .14 & .11 \\
\hline 9 & & & & & .01 & .02 & .05 & .14 & .37 & .20 & .20 \\
\hline 10 & & & & & & .01 & .03 & .11 & .35 & .23 & .27 \\
\hline
\end{tabular}

Figure C.4: Example overlap matrix output provided by the alchemical_analysis.py package for the $\mathrm{m}^{6} \mathrm{~A}$ duplex free energy simulations in the L-J leg [209]. The matrix indicates good phase-space overlap for our $\lambda$ schedule, which implies that large uncertainties or contradictory results are probably caused by insufficient sampling or unequilibrated data. 

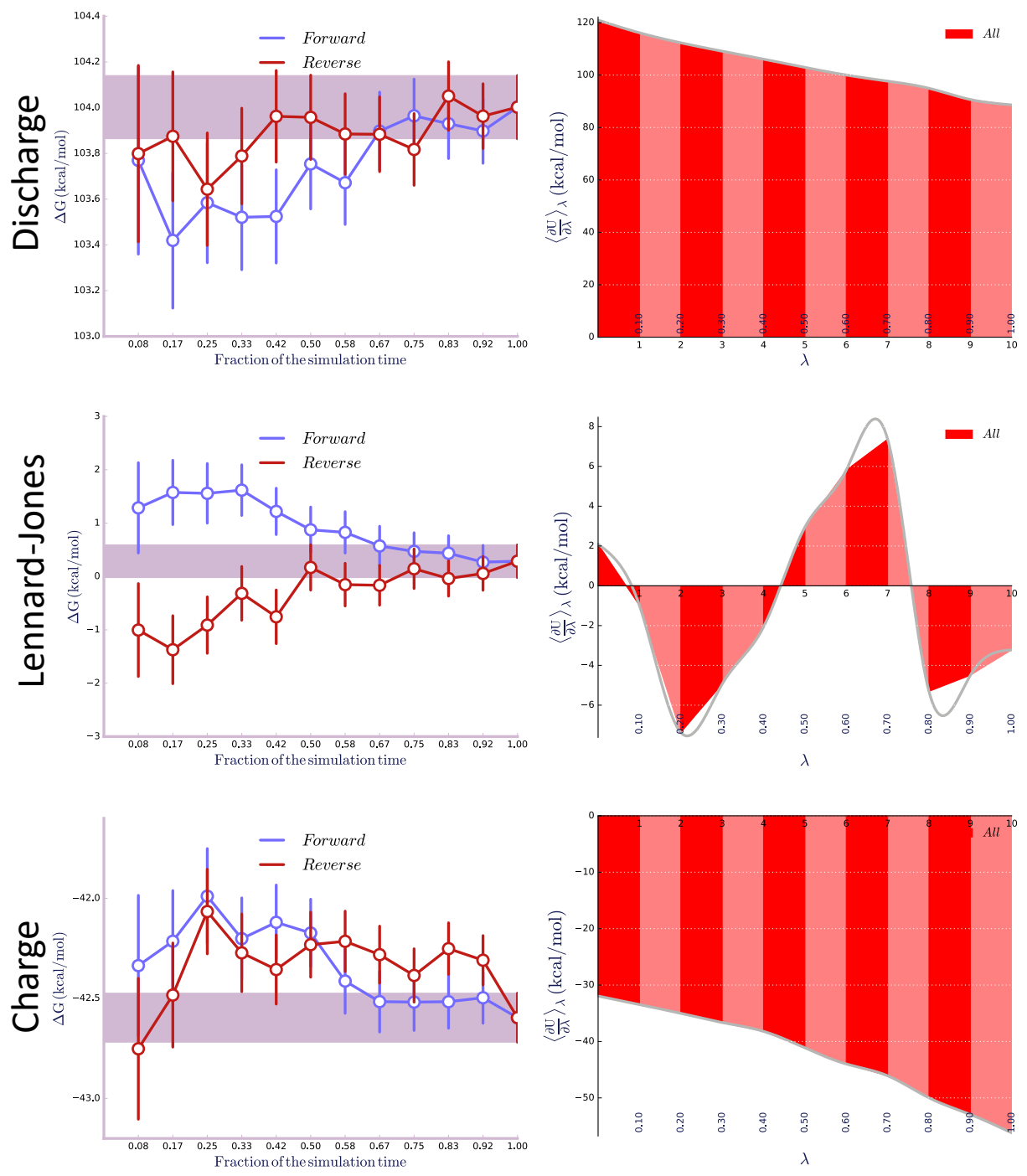

Figure C.5: Example output provided for $\mathrm{m}^{6} \mathrm{~A}$ duplex free energy simulations by the alchemical_analysis.py package for discharge, LJ, and charge legs of the thermodynamic cycle in the top, middle, and bottom panels, respectively [209]. (A) In the left panels, the convergence plots are displayed. The discharge and charge plots show rapid convergence of the estimated change in free energy while the LJ plot shows insufficient sampling, longterm correlations, and/or possibly unequilibrated data. (B) In the right panels, the $\left\langle\frac{\partial U}{\partial \lambda}\right\rangle_{\lambda}$ vs. $\lambda$ plots are shown with filled, red areas (silver curves) indicating the estimate from TI-Trapezoid (TI-Cubic) interpolation. 


\section{C.12 Analyzing mechanisms causing free energy changes}

Structural changes that occur during the simulation may impact the validity of the free energy results. Generally, we find small structural changes that may point toward mechanisms driving the free energy changes, but we do not find large structural changes that would call into question the validity of the free energy estimations.

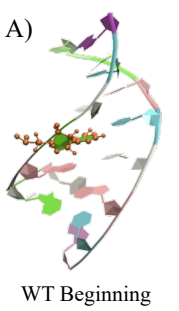

F)

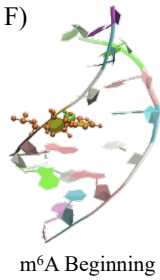

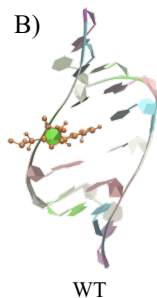

G)

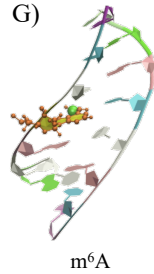

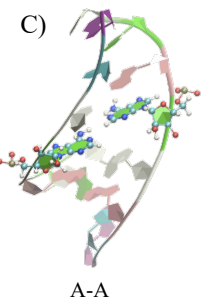

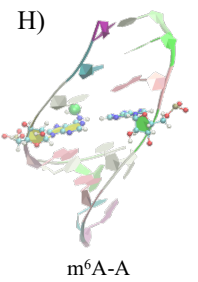

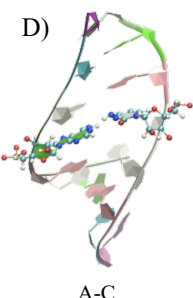

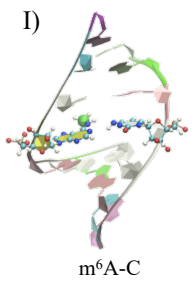

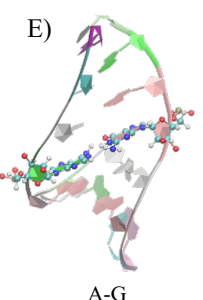

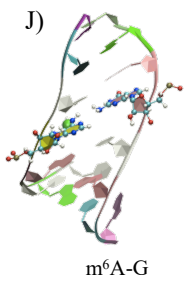

Figure C.6: Comparing final structures to initial topology in production simulations. A) At the beginning of a WT production simulation, the structure is in a standard A-form duplex. B) By the end of the simulation, the duplex structure is relatively unchanged. C) Even when the $12 \mathrm{U}$ residue is fully mutated to $12 \mathrm{~A}$, the overall duplex structure is stable. Rather than bulging, the mutated residue appears to turn its sugar edge toward 5A in response to the steric strain created by an incompatible purine-purine mutation. D) Mutating $12 \mathrm{U}$ to $12 \mathrm{C}$ does not appear to greatly affect the orientation of the nucleotide or the duplex topology. The Watson-Crick A-C base pair is not stable, having only one hydrogen bond between the amino group of $12 \mathrm{C}$ and the ring nitrogen of $5 \mathrm{~A}$. E) When $12 \mathrm{U}$ is mutated to $12 \mathrm{G}$, a Watson-Crick-oriented A-G base pair is formed through two hydrogen bonds between the $5 \mathrm{~A}$ and $12 \mathrm{G}$ ring nitrogen groups and the $5 \mathrm{~A}$ amino and $12 \mathrm{G}$ oxygen groups. F) The bottom row shows the methylated version. The methylation does not appear to greatly affect the topology. However, the alchemical free energy calculation indicates that $\mathrm{H}$ ) the $\mathrm{m}^{6} \mathrm{~A}-\mathrm{A}$ duplex is more stable than $\mathrm{C}$ ) the unmodified $\mathrm{A}-\mathrm{A}$ mutation. The amino group does not engage in base-pairing interactions in the A-A mutation, so the duplex structure is probably stabilized through enhancing stacking with $6 \mathrm{C}$ provided by methylation. Enhanced stability in the $\mathrm{m}^{6} \mathrm{~A}-\mathrm{A}$ context relative to the A-A context is consistent with the experimental twostate fit but not the van't Hoff calculation. 


\section{C.13 A "spring-loaded" modification}

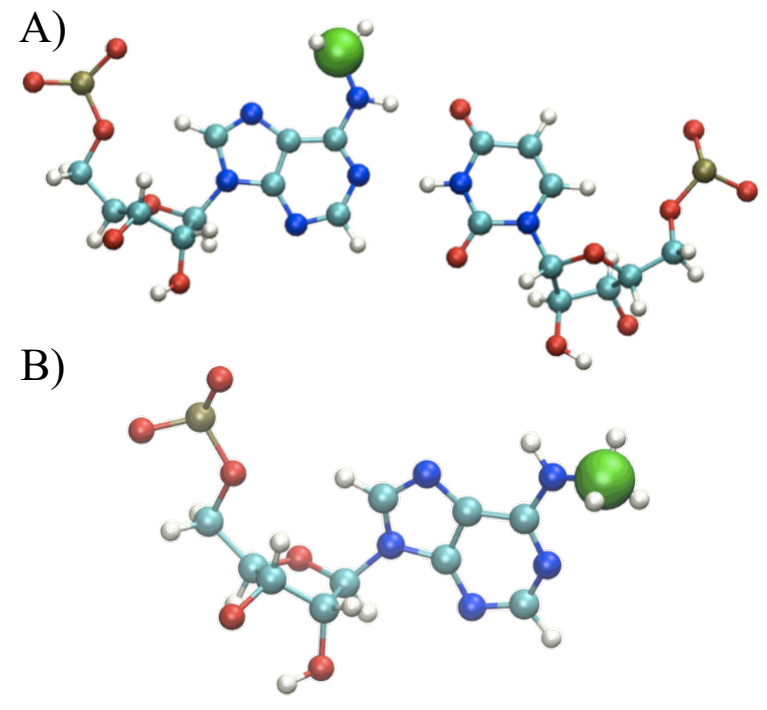

Figure C.7: Comparing the A) duplex arrangement of atoms to the B) single stranded arrangement shows that the methyl group (large, green bead) rotates to a higher energy, sterically strained orientation to facilitate hydrogen bonding in the pairing context. 


\section{C.14 PMFs}

The RMSD/E2E PMFs for all the simulated hairpins and analysis for the structures not included in the main text are shown below. Analysis of the summary structures included in Fig. 6.5 are found in the main text.

\section{C.14.1 WT}

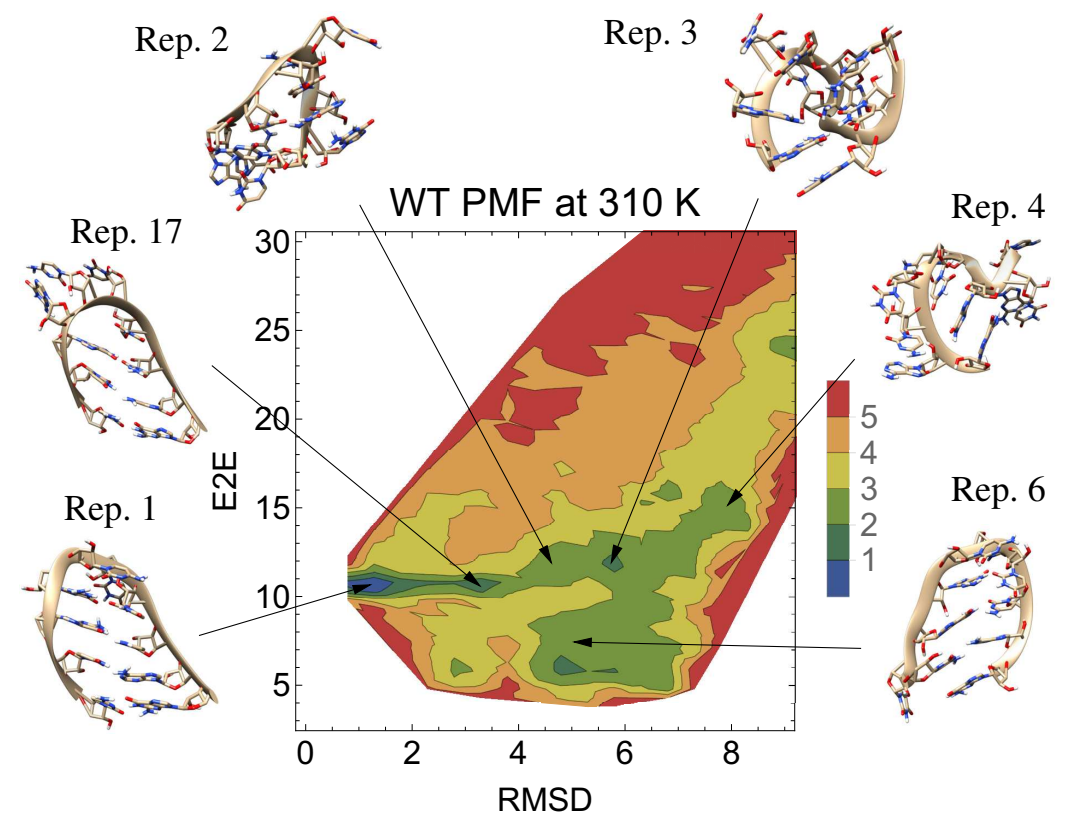

Figure C.8: The PMF for the WT hairpin along the end-to-end distance (E2E) and RMSD reaction coordinates indicates that unfolding preferentially begins at the loop end, with $\mathrm{NH}$ E2E distance retained until larger RMSDs are sampled. 


\section{C.14.2 $2 \mathrm{~m}^{6} \mathrm{~A}$}

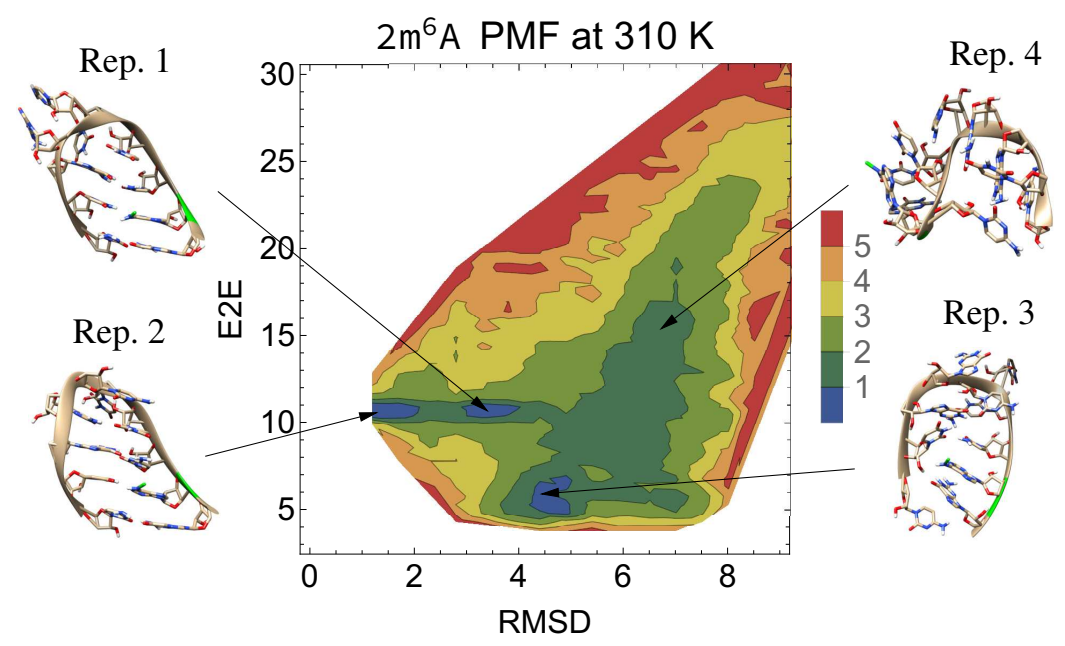

Figure C.9: The PMF for the $2 \mathrm{~m}^{6} \mathrm{~A}$ hairpin along the E2E and RMSD reaction coordinates indicates that unfolding still preferentially begins at the loop end, but unfolding from the terminal end is more energetically favorable than in the WT due to destabilization of the base pair between the $2 \mathrm{~m}^{6} \mathrm{~A}$ and $9 \mathrm{U}$. The ribbon and methyl of the modified nucleotide are highlighted in green in the representative frames. 


\section{C.14.3 $3 \mathrm{~m}^{4} \mathrm{C}$}

$\mathrm{N}^{4}$-methylcytosine $\left(\mathrm{m}^{4} \mathrm{C}\right)$ modification of hairpin residue $3 \mathrm{C}\left(3 \mathrm{~m}^{4} \mathrm{C}\right)$ results in stabilization of conformations where the $\mathrm{m}^{4} \mathrm{C}$ nucleotide engages in stacking interactions (see Fig. C.10). Furthermore, the weakening of the base pair between $3 \mathrm{~m}^{4} \mathrm{C}$ and $8 \mathrm{G}$ due to the methylation results in a broad swath of higher RMSD structures with relatively lower free energy that kinetically compete with the native hairpin (NH) structure. Similar to the WT case, the PMF proceeds from a NH conformation (Rep.1) through a slightly deepened metastable state (Rep. 14), relative to the WT. After accessing the metastable conformation, the path of least action rapidly degenerates into a multitude of random coil conformations. The representative structures from these random coil conformations (Reps. 2-5) indicate that the methylation enhances stacking interactions. Furthermore, the weakening of the base pair between nucleotides three and eight broadens the ability of the RNA to access extended states, relative to the WT, and the path of least action to an extended state is spread throughout a broader range of RMSDs, which indicates that melting of the stem more often proceeds from the loop-proximal end. The energy barrier separating the $\mathrm{NH}$ and $\mathrm{RC}$ conformations is slightly lowered to about $2.3 \mathrm{kcal} / \mathrm{mol}$. 


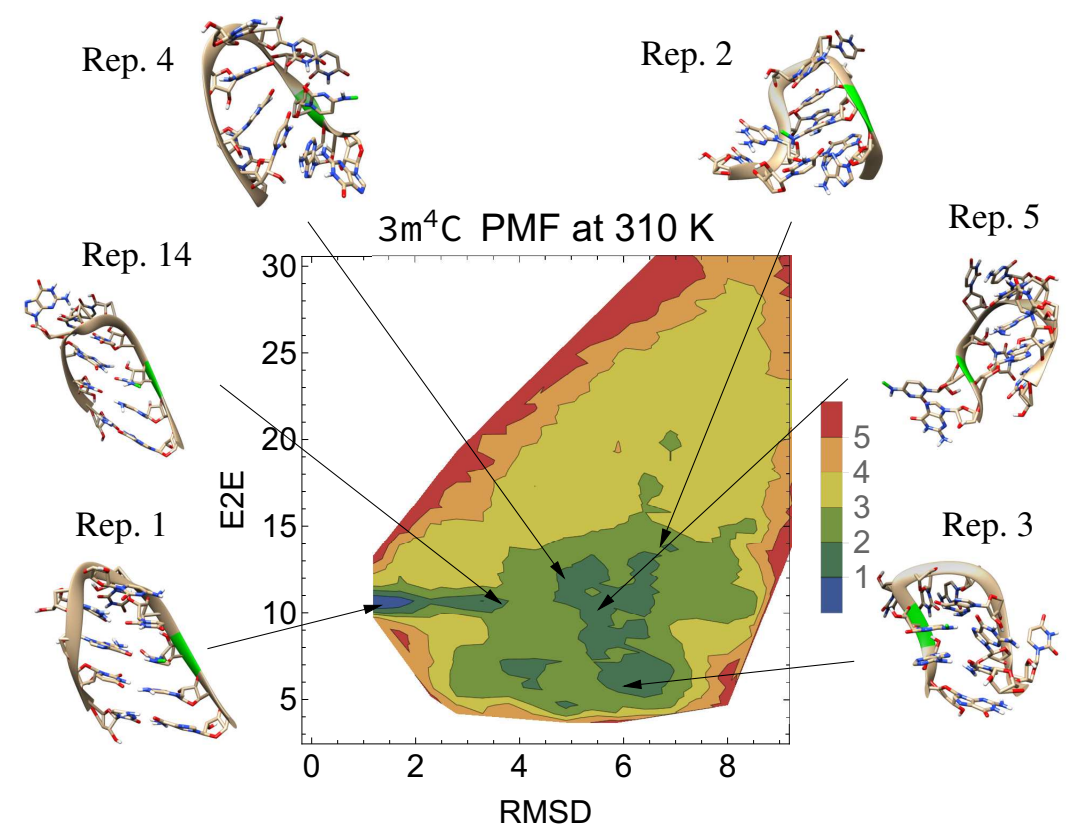

Figure C.10: The PMF for the $3 \mathrm{~m}^{4} \mathrm{C}$ hairpin along the E2E and RMSD reaction coordinates indicates that unfolding still preferentially begins at the loop end, but this modification facilitates sampling of collapsed structures as is evidenced by the lack of low energy states with high E2E and the myriad of low energy states with lower E2E but high RMSD. 


\section{C.14.4 $4 \mathrm{~m}^{5} \mathrm{U}$}

Ribothymidine $\left(\mathrm{m}^{5} \mathrm{U}\right)$ modification of $4 \mathrm{U}$ in the hairpin $\left(4 \mathrm{~m}^{5} \mathrm{U}\right)$ somewhat destabilizes the hairpin by promoting the sampling of higher RMSD and E2E conformations (see Fig. C.11). While other hairpin derivatives appear to unfold due disruption of the tetraloop that leads to sheering forces along the helical axis causing a preponderance of stacking, high RMSD, low E2E distance conformations, $4 \mathrm{~m}^{5} \mathrm{U}$ enables sampling of higher E2E distance conformations while remaining lower RMSD, as evidenced by the appearance of cluster 4 (Rep. 4). This cluster probably results from stabilization of the stacking interaction between $3 \mathrm{C}$ and $4 \mathrm{U}$, which allows the terminal nucleotides to grow farther apart while retaining structure in the loop region. While $4 \mathrm{U}$ and $7 \mathrm{G}$ can form a base pairing interaction, which is probably stabilized by the electron donating methyl group, the enhancement of stacking interactions in collapsed and extended coil conformations (Reps. 2-3 and 5-6) due to the methylation appears to compete with the stabilization of this highly sterically constrained base pairing interaction. The increased stability of the tetraloop appears to create a small energy barrier between near-NH conformations (Reps. 1 and 4) and extended coil conformations. However, once the tetraloop structure is disrupted, the methylation also stabilizes random coil, single-stranded stacking interactions. The energy barrier separating the $\mathrm{NH}$ and $\mathrm{RC}$ states is about $2.6 \mathrm{kcal} / \mathrm{mol}$. 


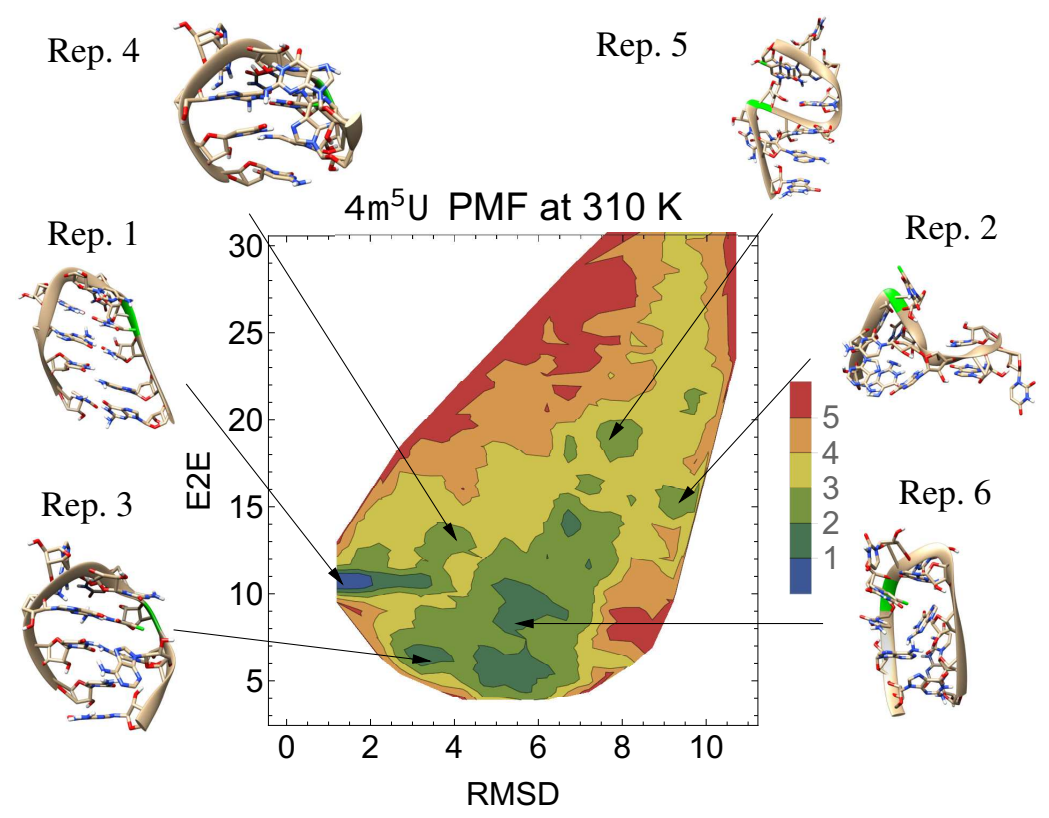

Figure C.11: The PMF for the $4 \mathrm{~m}^{5} \mathrm{U}$ hairpin along the E2E and RMSD reaction coordinates indicates that unfolding may start from the terminal end, unlike in the WT case. 


\section{C.14.5 $5 \mathrm{~m}^{5} \mathrm{U}$}

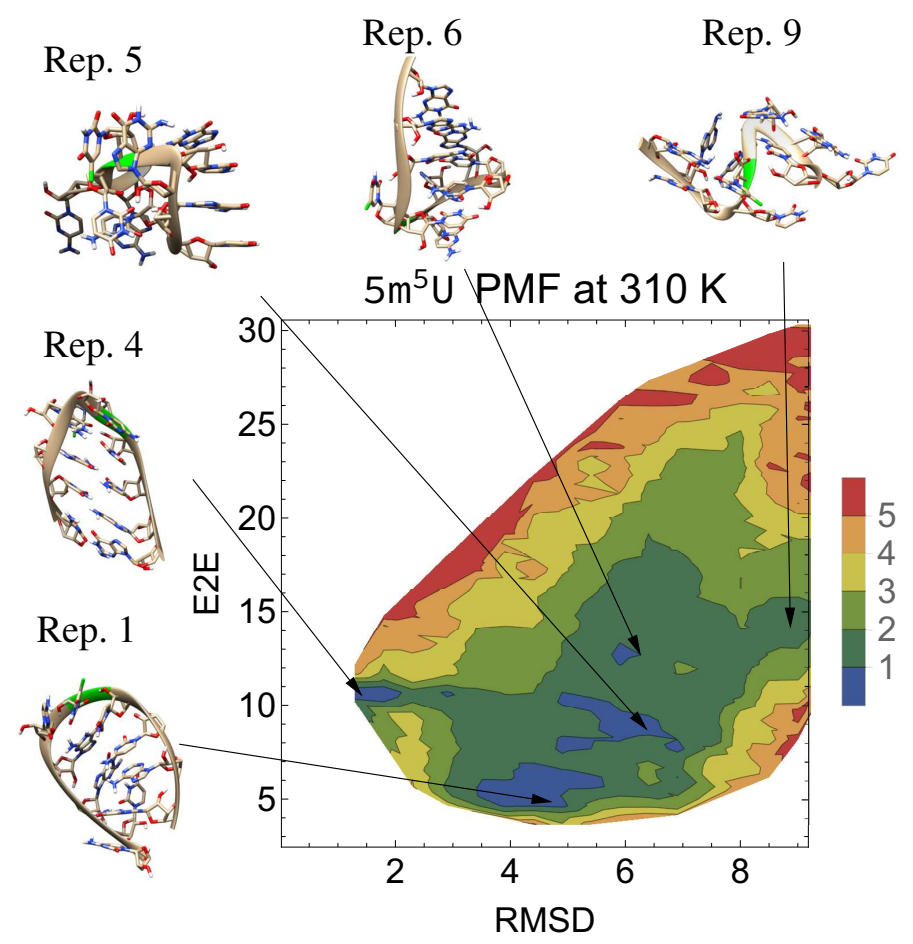

Figure C.12: The PMF for the $5 \mathrm{~m}^{5} \mathrm{U}$ hairpin along the E2E and RMSD reaction coordinates shows that this loop modification can markedly disrupt the NH structure. 


\section{C.14.6 $6 \mathrm{~m}^{4} \mathrm{C}$}

Similar to $5 \mathrm{~m}^{5} \mathrm{U}, \mathrm{m}^{4} \mathrm{C}$ modification of $6 \mathrm{C}$ in the hairpin $\left(6 \mathrm{~m}^{4} \mathrm{C}\right)$ destabilizes the $\mathrm{NH}$ conformation relative to the WT by stabilizing the metastable intermediate structure through stacking interactions (Rep. 2); the representative structure from cluster 17 in Fig. C.8 also shows 6C stacking in the metastable state (see Fig. C.13). In the folded NH conformation (Rep. 1), $6 \mathrm{~m}^{4} \mathrm{C}$ engages in no obvious interactions with other nucleotides and is pointed towards solution on the major groove side of the helix. Like $5 \mathrm{~m}^{5} \mathrm{U}, 6 \mathrm{~m}^{4} \mathrm{C}$ also stabilizes collapsed conformations through stacking interactions. The $\mathrm{NH}$ conformation is much more stable in this case than with $5 \mathrm{~m}^{5} \mathrm{U}$ because $\mathrm{m}^{4} \mathrm{C}$ is already on the major groove side, where stacking interactions are more easily accessed and refolding to the $\mathrm{NH}$ requires less large-scale movement of other nucleotides. Once the metastable state is achieved, unfolding can proceed from the terminal end (Rep. 6) or directly sample collapsed conformations (Rep. 3), neither of which is energetically favorable in the WT case, where the path of least action along the E2E coordinate did not facilitate sampling of larger or smaller E2E conformations until higher RMSDs were achieved. The energy barrier separating NH and $\mathrm{RC}$ conformations is about $2.7 \mathrm{kcal} / \mathrm{mol}$. 


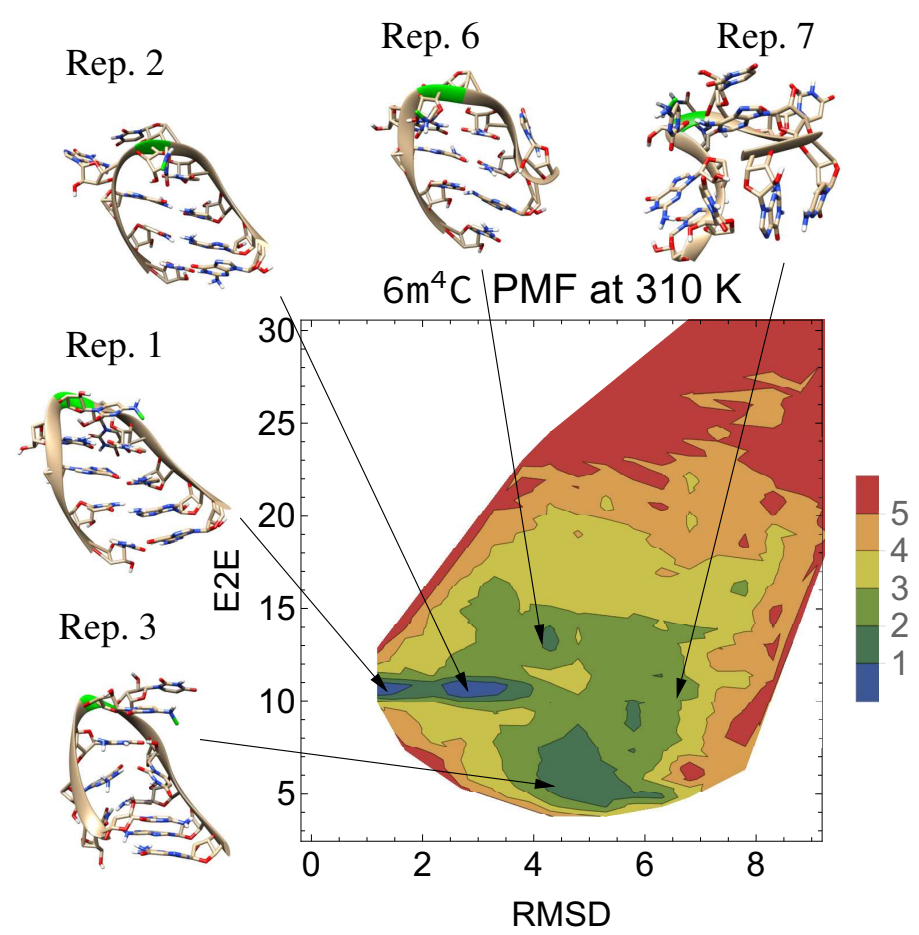

Figure C.13: The PMF for the $6 \mathrm{~m}^{4} \mathrm{C}$ hairpin along the E2E and RMSD reaction coordinates shows that this loop modification facilitates unfolding from the terminal end in comparison to the WT. 


\section{C.14.7 $7 \mathbf{m}^{2} \mathbf{G}$}

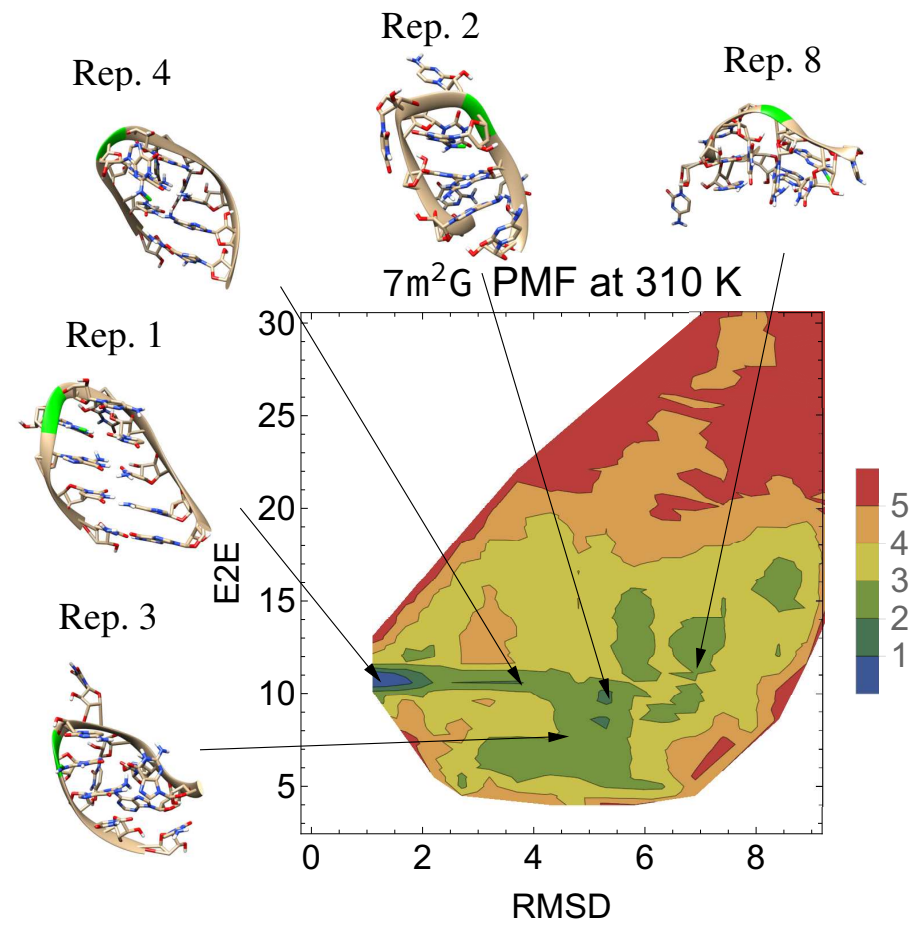

Figure C.14: The PMF for the $7 \mathrm{~m}^{2} \mathrm{G}$ hairpin along the E2E and RMSD reaction coordinates shows that this loop modification destabilizes the metastable state and prevents sampling of high E2E conformations. 


\section{C.14.8 $8 \mathrm{~m}^{2} \mathrm{G}$}

While $\mathrm{m}^{2} \mathrm{G}$ modification of $8 \mathrm{G}$ in the hairpin $\left(8 \mathrm{~m}^{2} \mathrm{G}\right)$ does not greatly stabilize or destabilize the hairpin, interesting changes to the RMSD/E2E PMF occur when this nucleotide is modified (see Fig. C.15). The metastable state seen in the WT RMSD/E2E PMF has disappeared, but the random coil conformations appear to be stabilized by this methylation (Reps. 2-4 and 6). Disappearance of the metastable structure indicates that this modification casts the energy landscape into an easily definable two state system. Stabilization of the hairpin from the enhanced stacking due to methylation with $7 \mathrm{G}$ appears to compete with destabilization of the hairpin due to weakening of the base pairing interactions between the 3C and 8G. Calculation of the free energy change due to modification appears to slightly favor $8 \mathrm{~m}^{2} \mathrm{G}$ over the WT form of the RNA for stabilizing the hairpin. This slight preference for the hairpin is probably due to the disappearance of the metastable state from the PMF and the stacking stability provided by the methylation. Additionally, this methylation does not completely deny hydrogen bonding of the $\mathrm{N}^{2}$ functional group with its base pairing partner, which allows base pair formation, although base pairing is less favorable than in the WT case. The energy barrier separating NH and RC states is about $3.3 \mathrm{kcal} / \mathrm{mol}$. 


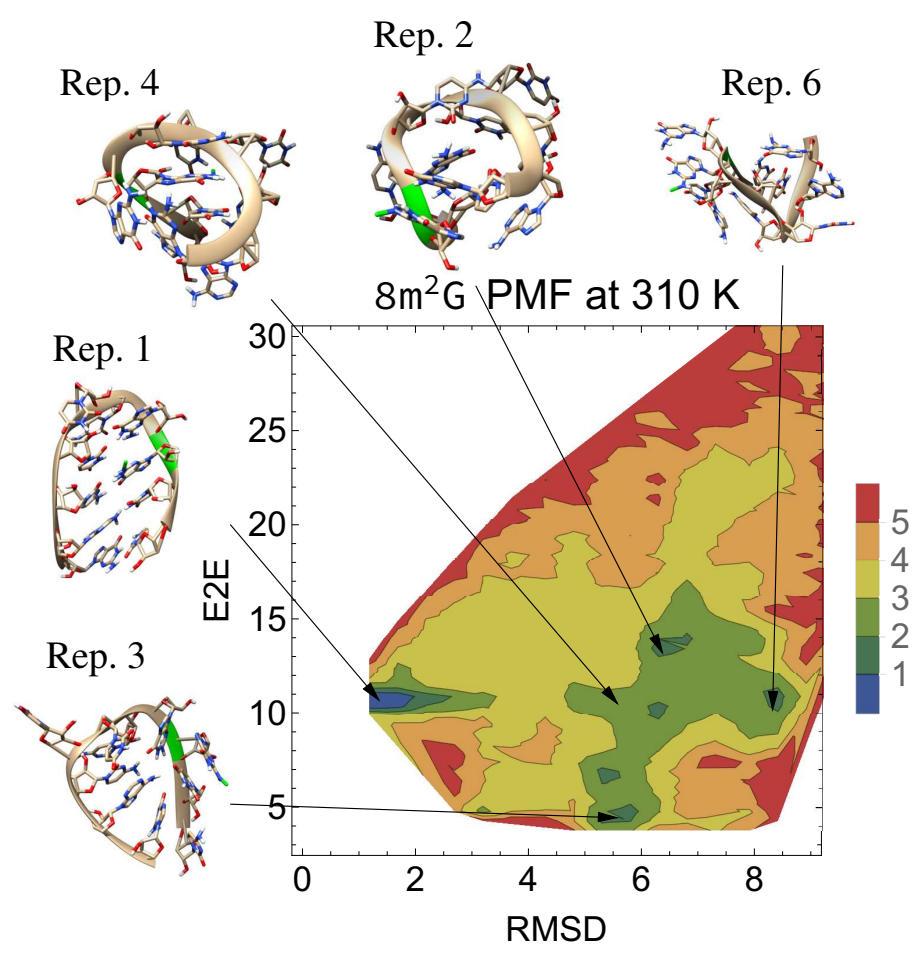

Figure C.15: The PMF for the $8 \mathrm{~m}^{2} \mathrm{G}$ hairpin along the $\mathrm{E} 2 \mathrm{E}$ and $\mathrm{RMSD}$ reaction coordinates shows that this modification removes the metastable state and slightly stabilizes the $\mathrm{NH}$ conformation. 


\section{C.14.9 $9 \mathrm{~m}^{5} \mathrm{U}$}

When imposed on $9 \mathrm{U}$ of the hairpin $\left(9 \mathrm{~m}^{5} \mathrm{U}\right), \mathrm{m}^{5} \mathrm{U}$ results in a PMF (Fig. C.16) that is qualitatively similar to the $2 \mathrm{~m}^{6} \mathrm{~A}$ PMF (see Fig. C.9). Both stabilize the metastable state (Rep. 4 in Fig. C.16) and collapsed random coil conformations (Rep. 8), relative to the WT. Interestingly, the metastable state appears to shift to a lower RMSD conformation for $9 \mathrm{~m}^{5} \mathrm{U}$, relative to both $2 \mathrm{~m}^{6} \mathrm{~A}$ and the WT. Apparently, highly extended conformations are less favorable for $9 \mathrm{~m}^{5} \mathrm{U}$ than for $2 \mathrm{~m}^{6} \mathrm{~A}$, but moderately extended conformations with lower RMSD are more favorable, seen on the PMF above and between Reps. 3 and 4. Additionally, lower RMSD collapsed conformations are not as favorable for $9 \mathrm{~m}^{5} \mathrm{U}$ as $2 \mathrm{~m}^{6} \mathrm{~A}$. In general, the $\mathrm{NH}$ state is more favorable for $9 \mathrm{~m}^{5} \mathrm{U}$ than $2 \mathrm{~m}^{6} \mathrm{~A}$ because this modification does not impede base pairing. The energy barrier separating the $\mathrm{NH}$ and $\mathrm{RC}$ states is about $2.3 \mathrm{kcal} / \mathrm{mol}$. 


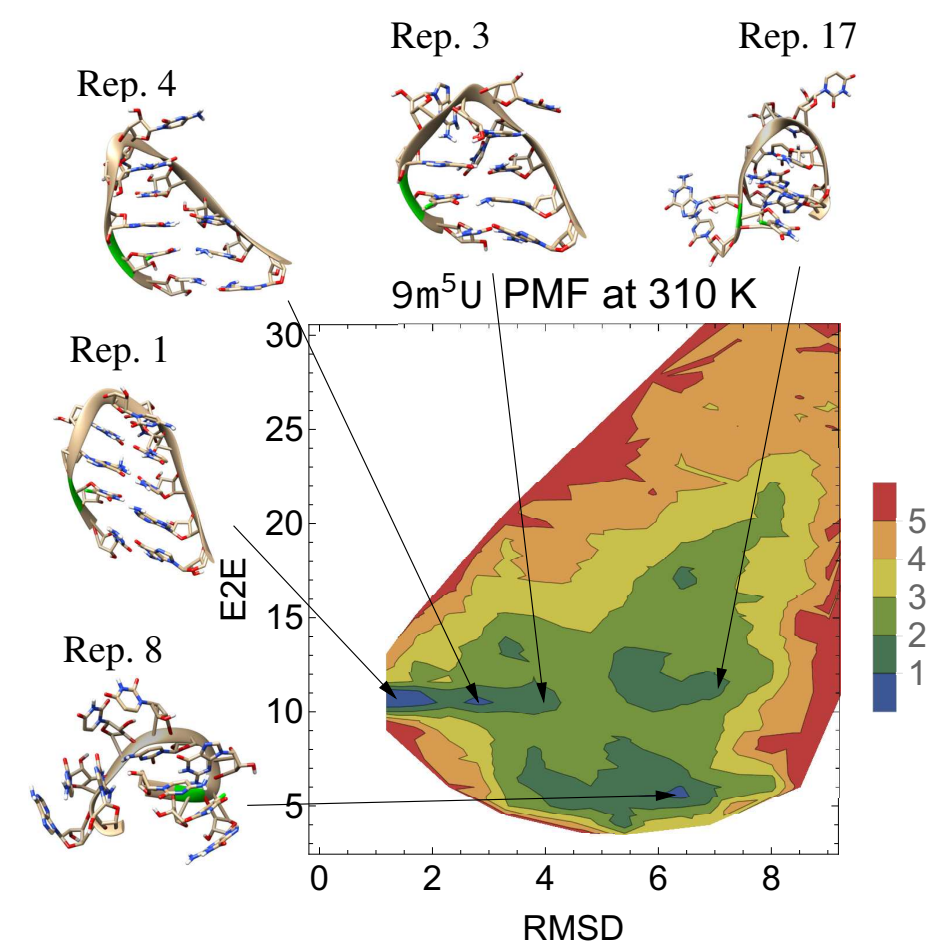

Figure C.16: The PMF for the $9 \mathrm{~m}^{5} \mathrm{U}$ hairpin along the E2E and RMSD reaction coordinates shows that this modification removes the metastable state and slightly stabilizes the $\mathrm{NH}$ conformation. 


\section{Bibliography}

[1] Zhichao Miao and Eric Westhof. "RNA Structure: Advances and Assessment of 3D Structure Prediction”. In: Annu. Rev. Biophys. 46.1 (2017), pp. 483-503. DOI: 10.1146/annurev-biophys-070816-034125. URL: https: // doi . org/10.1146/annurev-biophys-070816-034125.

[2] Christian Laing and Tamar Schlick. "Computational approaches to 3D modeling of RNA.” In: J. Phys. Condens. Matter 22.28 (2010), p. 283101. ISSN: 1361-648X (Electronic). DOI: 10.1088/0953-8984/22/28/283101.

[3] Bruce A Shapiro et al. "Bridging the gap in RNA structure prediction." In: Curr. Opin. Struct. Biol. 17.2 (2007), pp. 157-165. ISSN: 0959-440X (Print). DOI: 10 . 1016/j.sbi.2007.03.001.

[4] Sarah A Woodson and Stephen M Mount. "The RNA World, second edition, edited by Raymond F. Gesteland, Thomas R. Cech, and John F. Atkins. 1999. Cold Spring Harbor, New York: Cold Spring Harbor Laboratory Press. Hardcover, 709 pp. 129.” In: RNA 5.9 (1999), pp. 1133-1134. DOI: $10.1017 /$ S1355838299001259. 
[5] John S Mattick and Igor V Makunin. "Non-coding RNA." In: Hum. Mol. Genet. 15 Spec No (2006), R17-29. ISSN: 0964-6906 (Print). DOI: 10 . 1093 / hmg / ddl046.

[6] W K Johnston et al. "RNA-catalyzed RNA polymerization: accurate and general RNA-templated primer extension.” In: Science 292.5520 (2001), pp. 1319-1325. ISSN: 0036-8075 (Print). DOI: $10.1126 /$ science. 1060786.

[7] Aniela Wochner et al. "Ribozyme-Catalyzed Transcription of an Active Ribozyme". In: Science (80-. ). 332.6026 (2011), pp. 209-212. ISSN: 0036-8075. DOI: 10 . 1126/science.1200752. URL: https://science. sciencemag . org/content/332/6026/209.

[8] Jane S Richardson et al. "RNA backbone: consensus all-angle conformers and modular string nomenclature (an RNA Ontology Consortium contribution)." In: RNA 14.3 (2008), pp. 465-481. ISSN: 1469-9001 (Electronic). DOI: 10 . 1261/ rna. 657708.

[9] Huanwang Yang et al. "Tools for the automatic identification and classification of RNA base pairs". In: Nucleic Acids Res. 31.13 (2003), pp. 3450-3460. ISSN: 1362-4962. DOI: 10 . 1093 / nar/gkg529. URL: https: / / pubmed. ncbi . nlm.nih.gov/12824344https://www.ncbi.nlm.nih.gov/pmc/ articles/PMC168936/.

[10] Sébastien Lemieux and François Major. "RNA canonical and non-canonical base pairing types: a recognition method and complete repertoire." In: Nucleic Acids Res. 30.19 (2002), pp. 4250-4263. ISSN: 1362-4962 (Electronic). DOI: 10 . $1093 /$ nar/gkf540. 
[11] Ruxandra I Dima, Changbong Hyeon, and D Thirumalai. "Extracting stacking interaction parameters for RNA from the data set of native structures." In: J. Mol. Biol. 347.1 (2005), pp. 53-69. ISSN: 0022-2836 (Print). DOI: 10.1016 / j · jmb . 2004.12 .012 .

[12] Evgeny Nudler and Alexander S Mironov. "The riboswitch control of bacterial metabolism.” In: Trends Biochem. Sci. 29.1 (2004), pp. 11-17. ISSN: 0968-0004 (Print). DOI: $10.1016 / j . t i b s .2003 .11 .004$.

[13] Brian J Tucker and Ronald R Breaker. "Riboswitches as versatile gene control elements.” In: Curr. Opin. Struct. Biol. 15.3 (2005), pp. 342-348. ISSN: 0959-440X (Print). DOI: $10.1016 / j \cdot s b i .2005 .05 .003$.

[14] Xiaojun Xu and Shi-Jie Chen. "Kinetic mechanism of conformational switch between bistable RNA hairpins.” In: J. Am. Chem. Soc. 134.30 (2012), pp. 1249912507. ISSN: 1520-5126 (Electronic). DOI: $10.1021 /$ ja3013819.

[15] Song Cao and Shi-Jie Chen. "Predicting RNA folding thermodynamics with a reduced chain representation model.” In: RNA 11.12 (2005), pp. 1884-1897. ISSN: 1355-8382 (Print). DOI: $10.1261 /$ rna. 2109105.

[16] Song Cao and Shi-Jie Jie Chen. "Physics-Based De Novo Prediction of RNA 3D Structures”. In: J. Phys. Chem. B 115.14 (2011), pp. 4216-4226. ISSN: 15205207. DOI: 10 . 1021/jp112059y. URL: https : / / doi .org/10 .1021/ jp112059y.

[17] Marc Parisien and François Major. "The MC-Fold and MC-Sym pipeline infers RNA structure from sequence data”. In: Nature 452.7183 (2008), pp. 51-55. ISSN: 
1476-4687. DOI: 10.1038 / nature06684. URL: https://doi .org/10. 1038 / nature06684.

[18] François Major. "Building three-dimensional ribonucleic acid structures". In: Comput. Sci. Eng. 5.5 (2003), pp. 44-53. ISSN: 1558-366X. DOI: 10 .1109/MCISE . 2003.1225860 .

[19] Neocles B Leontis, Aurelie Lescoute, and Eric Westhof. "The building blocks and motifs of RNA architecture." In: Curr. Opin. Struct. Biol. 16.3 (2006), pp. 279-287. ISSN: 0959-440X (Print). DOI: 10.1016/j.sbi.2006.05.009.

[20] Rhiju Das and David Baker. "Automated de novo prediction of native-like RNA tertiary structures". In: Proc. Natl. Acad. Sci. 104.37 (2007), pp. 14664-14669. ISSN: 0027-8424. DOI: 10.1073 /pnas . 0703836104 . URL: https: / /www . pnas.org/content/104/37/14664.

[21] Samuela Pasquali and Philippe Derreumaux. "HiRE-RNA: A High Resolution CoarseGrained Energy Model for RNA”. In: J. Phys. Chem. B 114.37 (2010), pp. $11957-$ 11966. ISSN: 1520-6106. DOI: 10 . 1021/ jp102497y. URL: https:// doi . org/10.1021/jp102497y.

[22] Clarence Y Cheng et al. "RNA structure inference through chemical mapping after accidental or intentional mutations." In: Proc. Natl. Acad. Sci. U. S. A. 114.37 (2017), pp. 9876-9881. ISSN: 1091-6490 (Electronic). DOI: 10 . 1073 / pnas . 1619897114.

[23] Sichun Yang et al. "RNA Structure Determination Using SAXS Data". In: J. Phys. Chem. B 114.31 (2010), pp. 10039-10048. ISSN: 1520-6106. DOI: $10.1021 /$ jp1057308. URL: https://doi.org/10.1021/jp1057308. 
[24] Marc Parisien and François Major. "Determining RNA three-dimensional structures using low-resolution data”. In: J. Struct. Biol. 179.3 (2012), pp. 252-260. ISSN: 1047-8477. DOI: https : / / doi .org/10.1016/j.jsto. 2011.12 . 024. URL: http://WWW. sciencedirect.com/science/article/ pii/S1047847712000627.

[25] Feng Ding et al. "Three-dimensional RNA structure refinement by hydroxyl radical probing.” In: Nat. Methods 9.6 (2012), pp. 603-608. ISSN: 1548-7105 (Electronic). DOI: $10.1038 /$ nmeth.1976.

[26] Zhen Xia et al. "RNA 3D structure prediction by using a coarse-grained model and experimental data.” In: J. Phys. Chem. B 117.11 (2013), pp. 3135-3144. ISSN: 1520-5207 (Electronic). DOI: $10.1021 /$ jp $400751 \mathrm{w}$.

[27] Edward J Merino et al. "RNA Structure Analysis at Single Nucleotide Resolution by Selective 2-Hydroxyl Acylation and Primer Extension (SHAPE)". In: J. Am. Chem. Soc. 127.12 (2005), pp. 4223-4231. ISSN: 0002-7863. DOI: 10 . $1021 /$ ja043822v. URL: https://doi.org/10.1021/ja043822v.

[28] Kevin A Wilkinson, Edward J Merino, and Kevin M Weeks. "Selective 2-hydroxyl acylation analyzed by primer extension (SHAPE): quantitative RNA structure analysis at single nucleotide resolution”. In: Nat. Protoc. 1.3 (2006), pp. 1610-1616. ISSN: 1750-2799. DOI: $10.1038 /$ nprot.2006.249. URL: https : / / doi . org/10.1038/nprot.2006.249.

[29] Byron Lee et al. "Comparison of SHAPE reagents for mapping RNA structures inside living cells". In: RNA 23.2 (2017), pp. 169-174. ISSN: 1469-9001. DOI: 10 . 1261/rna.058784.116. URL: https : / / pubmed.ncbi.nlm.nih. 
gov/27879433https://www.ncbi.nlm.nih.gov/pmc/articles/ PMC5238792/.

[30] Costin M Gherghe et al. "Strong Correlation between SHAPE Chemistry and the Generalized NMR Order Parameter (S2) in RNA". In: J. Am. Chem. Soc. 130.37 (2008), pp. 12244-12245. ISSN: 0002-7863. DOI: 10 . 1021 / ja 804541 s. URL: https://doi.org/10.1021/ja804541s.

[31] Kevin M Weeks. "Advances in RNA structure analysis by chemical probing." In: Curr. Opin. Struct. Biol. 20.3 (2010), pp. 295-304. ISSN: 1879-033X (Electronic). DOI: $10.1016 / j . s b i .2010 .04 .001$.

[32] Jennifer L McGinnis et al. "The Mechanisms of RNA SHAPE Chemistry". In: $J$. Am. Chem. Soc. 134.15 (2012), pp. 6617-6624. ISSN: 0002-7863. DOI: $10.1021 /$ ja2104075. URL: https://doi.org/10.1021/ja2104075.

[33] Katherine E Deigan et al. "Accurate SHAPE-directed RNA structure determination”. In: Proc. Natl. Acad. Sci. 106.1 (2009), pp. 97-102. ISSN: 0027-8424. DOI: 10.1073/pnas.0806929106. URL: https://www.pnas.org/content/ $106 / 1 / 97$.

[34] Justin T Low and Kevin M Weeks. "SHAPE-directed RNA secondary structure prediction”. In: Methods 52.2 (2010), pp. 150-158. ISSN: 1095-9130. DOI: 10 . 1016/j.ymeth.2010.06.007. URL: https://pubmed.ncbi.nlm. nih.gov/20554050https://www.ncbi.nlm.nih.gov/pmc/ articles/PMC2941709/.

[35] Wipapat Kladwang et al. "Understanding the errors of SHAPE-directed RNA structure modeling”. In: Biochemistry 50.37 (2011), pp. 8049-8056. ISSN: 1520-4995. 
DOI: 10 .1021/bi200524n. URL: https: / / pubmed.ncbi.nlm.nih . gov/21842868https://www.ncbi.nlm.nih.gov/pmc/articles/ $\mathrm{PMC} 3172344 /$.

[36] Christine E Hajdin et al. "Accurate SHAPE-directed RNA secondary structure modeling, including pseudoknots". In: Proc. Natl. Acad. Sci. U. S. A. 110.14 (2013), pp. 5498-5503. ISSN: 1091-6490. DOI: 10 . 1073 / pnas . 1219988110. URL: https://pubmed.ncbi.nlm.nih.gov/23503844https : / / www . ncbi.nlm.nih.gov/pmc/articles/PMC3619282/.

[37] Christopher W Leonard et al. "Principles for understanding the accuracy of SHAPEdirected RNA structure modeling”. In: Biochemistry 52.4 (2013), pp. 588-595. ISSN: 1520-4995. DOI: 10 . 1021/bi300755u. URL: https : / / pubmed . ncbi.nlm.nih.gov/23316814https://www.ncbi.nlm.nih.gov/ pmc/articles/PMC3578230/.

[38] Douglas H Turner and David H Mathews. "NNDB: the nearest neighbor parameter database for predicting stability of nucleic acid secondary structure." In: Nucleic Acids Res. 38.Database issue (2010), pp. D280-2. ISSN: 1362-4962 (Electronic). DOI: $10.1093 /$ nar/gkp892.

[39] Stefanie A Mortimer and Kevin M Weeks. "Time-resolved RNA SHAPE chemistry: quantitative RNA structure analysis in one-second snapshots and at singlenucleotide resolution.” In: Nat. Protoc. 4.10 (2009), pp. 1413-1421. ISSN: 17502799 (Electronic). DOI: $10.1038 /$ nprot . 2009.126. 
[40] Wipapat Kladwang et al. "A two-dimensional mutate-and-map strategy for noncoding RNA structure.” In: Nat. Chem. 3.12 (2011), pp. 954-962. ISSN: 1755-4349 (Electronic). DOI: $10.1038 /$ nchem.1176.

[41] Kady-Ann Steen, Greggory M Rice, and Kevin M Weeks. "Fingerprinting noncanonical and tertiary RNA structures by differential SHAPE reactivity." In: J. Am. Chem. Soc. 134.32 (2012), pp. 13160-13163. ISSN: 1520-5126 (Electronic). DOI: $10.1021 /$ ja304027m.

[42] Matthew J Smola et al. "SHAPE reveals transcript-wide interactions, complex structural domains, and protein interactions across the Xist lncRNA in living cells." In: Proc. Natl. Acad. Sci. U. S. A. 113.37 (2016), pp. 10322-10327. ISSN: 1091-6490 (Electronic). DOI: 10.1073/pnas.1600008113.

[43] Kyle E Watters et al. "Characterizing RNA structures in vitro and in vivo with selective 2'-hydroxyl acylation analyzed by primer extension sequencing (SHAPESeq).” In: Methods 103 (2016), pp. 34-48. ISSN: 1095-9130 (Electronic). DOI: 10 . $1016 / j \cdot y m e t h .2016 .04 .002$.

[44] Rosa Diaz-Toledano, Gloria Lozano, and Encarnacion Martinez-Salas. "In-cell SHAPE uncovers dynamic interactions between the untranslated regions of the foot-andmouth disease virus RNA.” In: Nucleic Acids Res. 45.3 (2017), pp. 1416-1432. ISSN: 1362-4962 (Electronic). DOI: $10.1093 /$ nar/gkw795.

[45] Meghan Zubradt et al. "DMS-MaPseq for genome-wide or targeted RNA structure probing in vivo”. In: Nat. Methods 14.1 (2017), pp. 75-82. ISSN: 1548-7105. DOI: 10.1038/nmeth.4057. uRL: https: // doi.org/10.1038/nmeth . 4057. 
[46] Serdal Kirmizialtin et al. "Integrating molecular dynamics simulations with chemical probing experiments using SHAPE-FIT.” In: Methods Enzymol. 553 (2015), pp. 215-234. ISSN: 1557-7988 (Electronic). DOI: 10 .1016/bs . mie. 2014 . 10.061 .

[47] Magdalena A Jonikas et al. "Coarse-grained modeling of large RNA molecules with knowledge-based potentials and structural filters.” In: RNA 15.2 (2009), pp. 189199. ISSN: 1469-9001 (Electronic). DOI: $10.1261 / \mathrm{rna} .1270809$.

[48] B R Brooks et al. "CHARMM: the biomolecular simulation program." In: J. Comput. Chem. 30.10 (2009), pp. 1545-1614. ISSN: 1096-987X (Electronic). DOI: 10 . $1002 / j \mathrm{jcc} .21287$.

[49] Romelia Salomon-Ferrer, David A Case, and Ross C Walker. "An overview of the Amber biomolecular simulation package". In: WIREs Comput. Mol. Sci. 3.2 (2013), pp. 198-210. DOI: 10.1002 /wcms .1121. URL: https: / / onlinelibrary . wiley.com/doi/abs/10.1002/wcms.1121.

[50] Scott A Hollingsworth and Ron O Dror. "Molecular Dynamics Simulation for All". In: Neuron 99.6 (2018), pp. 1129-1143. ISSN: 0896-6273. DOI: 10 . 1016 / j . neuron. 2018 .08 .011. URL: https: // doi .org/10.1016/j. neuron.2018.08.011.

[51] Alan A Chen and Angel E García. "High-resolution reversible folding of hyperstable RNA tetraloops using molecular dynamics simulations". In: Proc. Natl. Acad. Sci. 110.42 (2013), pp. 16820-16825. ISSN: 0027-8424. DOI: 10 . 1073/pnas . 1309392110. URL: https://www . pnas . org/ content/110/42/ 16820. 
[52] Melissa K Meinel and Florian Müller-Plathe. "Loss of Molecular Roughness upon Coarse-Graining Predicts the Artificially Accelerated Mobility of Coarse-Grained Molecular Simulation Models". In: J. Chem. Theory Comput. 16.3 (2020), pp. 14111419. ISSN: 1549-9618. DOI: 10 .1021/acs . jctc.9b00943. URL: https: //doi.org/10.1021/acs.jctc.9b00943.

[53] Dong Zhang and Shi-Jie Chen. "IsRNA: An Iterative Simulated Reference State Approach to Modeling Correlated Interactions in RNA Folding". In: J. Chem. Theory Comput. 14.4 (2018), pp. 2230-2239. ISSN: 1549-9626. DOI: $10.1021 /$ acs.jctc.7b01228. URL: https: // pubmed.ncbi.nlm.nih.gov/ 29499114 https: //www ncbi.nlm.nih.gov/pmc/articles / PMC6311103/.

[54] Yanan Yue, Jianzhao Liu, and Chuan He. "RNA N6-methyladenosine methylation in post-transcriptional gene expression regulation.” In: Genes Dev. 29.13 (2015), pp. 1343-1355. ISSN: 1549-5477 (Electronic). DOI: $10.1101 / \mathrm{gad} .262766$. 115.

[55] Emily M. Harcourt, Anna M. Kietrys, and Eric T. Kool. "Chemical and structural effects of base modifications in messenger RNA”. In: Nature 541 (2017), pp. 339346. ISSN: 0028-0836. DOI: 10.1038 / nature21351.

[56] Thomas Carell et al. "Structure and function of noncanonical nucleobases". In: Angew. Chem. Int. Ed. 51 (2012), pp. 7110-7131. ISSN: 14337851. DOI: 10 . 1002/anie.201201193. 
[57] Mark Helm et al. "The presence of modified nucleotides is required for cloverleaf folding of a human mitochondrial tRNA”. In: Nucleic Acids Res. 26.7 (1998), pp. 1636-1643. ISSN: 03051048. DOI: 10.1093/nar/26.7.1636.

[58] Mark Helm and Yuri Motorin. "Detecting RNA modifications in the epitranscriptome: Predict and validate”. In: Nat. Rev. Genet. 18 (2017), pp. 275-291. ISSN: 14710064. DOI: 10 .1038/nrg.2016.169. URL: http://dx.doi.org/ $10.1038 / \operatorname{mrg} .2016 .169$.

[59] Paul F Agris. "The importance of being modified: an unrealized code to RNA structure and function”. In: RNA 21 (2015), pp. 552-554. DOI: $10.1261 / \mathrm{rna}$. 050575.115.

[60] Adrian Gabriel Torres, Eduard Batlle, and Lluis Ribas de Pouplana. "Role of tRNA modifications in human diseases". In: Trends Mol. Med. 20.6 (2014), pp. 306-314. ISSN: 1471499X. DOI: 10.1016/j.molmed.2014.01.008.

[61] Gianluigi Lichinchi et al. "Dynamics of the human and viral $\mathrm{m}^{6} \mathrm{~A}$ RNA methylomes during HIV-1 infection of T cells”. In: Nat. Microbiol. 1.4 (2016), p. 16011. ISSN: 20585276. DOI: 10.1038/nmicrobiol.2016.11. URL: http:// dx.doi.org/10.1038/nmicrobiol.2016.11.

[62] Anastassia Gomez and Navtej Toor. "Selecting New RNA Crystal Contacts". In: Structure 26.9 (2018), pp. 1166-1167. ISSN: 18784186. DOI: $10.1016 /$ j. str. 2018.08 .009$.

[63] Ville Y.P. Väre et al. "Chemical and conformational diversity of modified nucleosides affects tRNA structure and function”. In: Biomolecules 7.29 (2017). ISSN: 2218273X. DOI: $10.3390 /$ biom7010029. 
[64] Martin Jinek et al. "A Programmable Dual-RNA-Guided Endonuclease in Adaptive Bacterial Immunity”. In: Science (80-. ). 337 (2012), pp. 816-822.

[65] Prashant Mali et al. "RNA-Guided Human Genome”. In: Science (80-. ). 339.6121 (2013), pp. 823-827.

[66] Dong Zhang et al. "Unified energetics analysis unravels SpCas9 cleavage activity for optimal gRNA design”. In: Proc. Natl. Acad. Sci. 116.18 (2019), 8693 LP 8698. DOI: 10 . 1073 / pnas . 1820523116 . URL: http : / / www . pnas . org/content/116/18/8693. abstract.

[67] Hao Yin et al. "Structure-guided chemical modification of guide RNA enables potent non-viral in vivo genome editing." In: Nat. Biotechnol. 35.12 (2017), pp. 11791187. ISSN: 1546-1696. DOI: $10.1038 /$ nbt . 4005 . URL: http : / / www . ncbi.nlm.nih.gov/pubmed/29131148.

[68] Ayal Hendel et al. "Chemically modified guide RNAs enhance CRISPR-Cas genome editing in human primary cells”. In: Nat. Biotechnol. 33.9 (2015), pp. 985-989. ISSN: 1546-1696. DOI: 10 . 1038 / nbt . 3290. URL: https : / / doi . org / $10.1038 /$ nbt. 3290 .

[69] Ian A Roundtree and Chuan He. "RNA epigenetics chemical messages for posttranscriptional gene regulation Messenger RNA modifications”. In: Curr. Opin. Chem. Biol. 30 (2016), pp. 46-51. DOI: $10.1016 /$ j . cbpa. 2015 . 10 . 024. URL: http://dx.doi.org/10.1016/j.cbpa.2015.10.024.

[70] Mirela Andronescu et al. "The Determination of RNA Folding Nearest Neighbor Parameters". In: RNA Seq. Struct. Funct. Comput. Bioinformatic Methods, Methods Mol. Biol. Ed. by Jan Gorodkin and Walter L. Ruzzo. Vol. 1097. New York, 2014. 
Chap. 3, pp. 45-70. ISBN: 978-1-62703-708-2. DOI: 10 . $1007 / 978-1-62703-$ 709-9. URL: http://link.springer.com/10.1007/978-1-62703$709-9$.

[71] Jessica S. Reuter and David H. Mathews. "RNAstructure: software for RNA secondary structure prediction and analysis". In: BMC Bioinformatics 11 (2010), p. 129. DOI: $10.1186 / 1471-2105-11-129$.

[72] Ronny Lorenz et al. "ViennaRNA Package 2.0". In: Algorithms Mol. Biol. 6.26 (2011). ISSN: 1748-7188. DOI: $10.1186 / 1748-7188-6-26$.

[73] Andrea Tanzer, Ivo L. Hofacker, and Ronny Lorenz. "RNA modifications in structure prediction Status quo and future challenges”. In: Methods 156 (2019), pp. 3239. ISSN: 10959130. DOI: $10.1016 / j \cdot y m e t h .2018 .10 .019$.

[74] F. A.P. Vendeix, A. M. Munoz, and P. F. Agris. "Free energy calculation of modified base-pair formation in explicit solvent: A predictive model”. In: RNA 15 (2009), pp. 2278-2287. ISSN: 1355-8382. DOI: $10.1261 /$ rna.1734309. URL: http: //rnajournal.cshlp.org/cgi/doi/10.1261/rna.1734309.

[75] Mohit Chawla et al. "An atlas of RNA base pairs involving modified nucleobases with optimal geometries and accurate energies". In: Nucleic Acids Res. 43.14 (2015), pp. 6714-6729. ISSN: 13624962. DOI: $10.1093 /$ nar/gkv606.

[76] Raviprasad Aduri et al. "AMBER force field parameters for the naturally occurring modified nucleosides in RNA”. In: J. Chem. Theory Comput. 3 (2007), pp. 14641475. ISSN: 15499618. DOI: $10.1021 /$ ct $600329 \mathrm{w}$. 
[77] You Xu et al. "Additive CHARMM force field for naturally occurring modified ribonucleotides". In: J. Comput. Chem. 37.10 (2016), pp. 896-912. ISSN: 1096987X. DOI: $10.1002 /$ jcc.24307. arXiv: arXiv:1011.1669v3.

[78] Travis Hurst et al. "Quantitative Understanding of SHAPE Mechanism from RNA Structure and Dynamics Analysis". In: J. Phys. Chem. B 122.18 (2018), pp. 47714783. ISSN: 1520-6106. DOI: 10 . $1021 /$ acs . jpcb. 8b00 0575. URL: https: //doi.org/10.1021/acs.jpcb.8b00575.

[79] Travis Hurst, Yuanzhe Zhou, and Shi-Jie Chen. "Analytical modeling and deep learning approaches to estimating RNA SHAPE reactivity from 3D structure". In: Commun. Inf. Syst. 19.3 (2019), pp. 299-319. DoI: https : / / dx . doi .org/ 10.4310/CIS.2019.v19.n3.a4. URL: https://www.intlpress . com/site/pub/pages / journals/items / cis / content/vols / 0019/0003/a004/index.php.

[80] Travis Hurst and Shi-Jie Chen. "Sieving RNA 3D Structures with SHAPE and Evaluating Mechanisms Driving Sequence-Dependent Reactivity Bias". In: J. Phys. Chem. B 125.4 (2021), pp. 1156-1166. ISSN: 1520-6106. DOI: 10.1021 / acs . jpcb. 0c11365. URL: https: / / doi . org/10 . 1021/acs . jpcb . $0 c 11365$.

[81] Travis Hurst et al. "A Bayes-inspired theory for optimally building an efficient coarse-grained folding force field". In: Commun. Inf. Syst. 21.1 (2021), pp. 65-83. DOI: https://dx.doi.org/10.4310/CIS.2021.v21.n1.a4.

[82] Travis Hurst and Shi-Jie Chen. "Deciphering nucleotide modification-induced structure and stability changes". In: RNA Biol. (2021), pp. 1-11. ISSN: 1547-6286. DOI: 
10.1080/15476286.2021.1882179. URL: https://doi.org/10. $1080 / 15476286.2021 .1882179$.

[83] Raymond F. Gesteland, Thomas Cech, and John Fuller Atkins. The RNA World: The Nature of Modern RNA Suggests a Prebiotic RNA World. 2nd. Cold Spring Harbor, NY: Cold Spring Harbor Laboratory Press, 1999. ISBN: 087969-589-7.

[84] Hui Lu and Jeffrey Skolnick. "A distance-dependent atomic knowledge-based potential for improved protein structure selection”. In: Proteins Struct. Funct. Bioinforma. 44.3 (2001), pp. 223-232. ISSN: 0887-3585. DOI: 10.1002 /prot. 1087. URL: https://doi.org/10.1002/prot.1087.

[85] William Humphrey, Andrew Dalke, and Klaus Schulten. "VMD: Visual molecular dynamics”. In: J. Mol. Graph. 14.1 (1996), pp. 33-38. ISSN: 0263-7855. DOI: https://doi.org/10.1016/0263-7855(96)00018-5. URL: http: //www.sciencedirect.com/science/article/pii/0263785596000185.

[86] James C Phillips et al. "Scalable molecular dynamics with NAMD." In: J. Comput. Chem. 26.16 (2005), pp. 1781-1802. ISSN: 0192-8651 (Print). DOI: 10 . $1002 /$ jec.20289.

[87] D.A. Case et al. “Amber 2017, University of California, San Francisco”. In: (2017). DOI: 10.13140/RG.2.2.36172.41606. URL: http://rgdoi.net/10. $13140 / \mathrm{RG} .2 .2 .36172 .41606$.

[88] William L. Jorgensen et al. "Comparison of simple potential functions for simulating liquid water". In: J. Chem. Phys. 79.2 (1983), pp. 926-935. ISSN: 00219606. DOI: 10 .1063/1.445869. URL: https : / / doi .org/10 .1063/1. 445869 . 
[89] Michael Zuker. "Mfold web server for nucleic acid folding and hybridization prediction”. In: Nucleic Acids Res. 31.13 (2003), pp. 3406-3415. ISSN: 1362-4962. DOI: 10.1093/nar/gkg595. URL: https://pubmed.ncbi.nlm.nih. gov/12824337https://www.ncbi.nlm.nih.gov/pmc/articles/ PMC169194/.

[90] Xiaojun Xu, Peinan Zhao, and Shi-Jie Jie Chen. "Vfold: A web server for RNA structure and folding thermodynamics prediction”. In: PLoS One 9.9 (2014), pp. 17. ISSN: 19326203. DOI: $10.1371 /$ journal . pone.0107504.

[91] Zsuzsanna Sükösd et al. "Evaluating the accuracy of SHAPE-directed RNA secondary structure predictions.” In: Nucleic Acids Res. 41.5 (2013), pp. 2807-2816. ISSN: 1362-4962 (Electronic). DOI: $10.1093 /$ nar/gks1283.

[92] T E 3rd Cheatham and M A Young. "Molecular dynamics simulation of nucleic acids: successes, limitations, and promise." In: Biopolymers 56.4 (2000), pp. 232 256. ISSN: 0006-3525 (Print). DOI: $10.1002 / 1097-0282$ (2000) 56:4<232: : AID-BIP $10037>3.0 . \mathrm{CO} ; 2-\mathrm{H}$.

[93] Chanin Tolson Woods and Alain Laederach. "Classification of RNA structure change by 'gazing' at experimental data." In: Bioinformatics 33.11 (2017), pp. 1647-1655. ISSN: 1367-4811 (Electronic). DOI: $10.1093 /$ bioinformatics/btx041.

[94] Stefanie A Mortimer and Kevin M Weeks. "A fast-acting reagent for accurate analysis of RNA secondary and tertiary structure by SHAPE chemistry." In: J. Am. Chem. Soc. 129.14 (2007), pp. 4144-4145. ISSN: 0002-7863 (Print). DOI: 10 . $1021 / j a 0704028$. 
[95] Richard Evans et al. "De novo structure prediction with deep-learning based scoring”. In: Annu. Rev. Biochem. (2018).

[96] Matt Spencer, Jesse Eickholt, and Jianlin Cheng. “A Deep Learning Network Approach to ab initio Protein Secondary Structure Prediction.” In: IEEE/ACM Trans. Comput. Biol. Bioinforma. 12.1 (2015), pp. 103-112. ISSN: 1557-9964 (Electronic). DOI: $10.1109 / \mathrm{TCBB} .2014 .2343960$.

[97] Rhys Heffernan et al. "Improving prediction of secondary structure, local backbone angles and solvent accessible surface area of proteins by iterative deep learning". In: Sci. Rep. 5.1 (2015), p. 11476. ISSN: 2045-2322. DOI: 10 . 1038 / srep11476. URL: https: / / doi .org/10.1038/srep11476.

[98] Sheng Wang et al. "Protein Secondary Structure Prediction Using Deep Convolutional Neural Fields”. In: Sci. Rep. 6.1 (2016), p. 18962. ISSN: 2045-2322. DOI: 10.1038/srep18962. URL: https://doi.org/10.1038/srep18962.

[99] Jian Zhou and Olga Troyanskaya. "Deep supervised and convolutional generative stochastic network for protein secondary structure prediction”. In: Int. Conf. Mach. Learn. PMLR. 2014, pp. 745-753.

[100] Sheng Wang et al. "Accurate De Novo Prediction of Protein Contact Map by UltraDeep Learning Model." In: PLoS Comput. Biol. 13.1 (2017), e1005324. ISSN: 1553-7358 (Electronic). DOI: $10.1371 /$ journal.pcbi. 1005324.

[101] Hongjian Li et al. "Improving AutoDock Vina Using Random Forest: The Growing Accuracy of Binding Affinity Prediction by the Effective Exploitation of Larger Data Sets”. In: Mol. Inform. 34.23 (2015), pp. 115-126. DoI: https : / / doi . 
org/10.1002/minf.201400132. URL: https:// onlinelibrary . wiley.com/doi/abs/10.1002/minf.201400132.

[102] Zixuan Cang and Guo-Wei Wei. "TopologyNet: Topology based deep convolutional and multi-task neural networks for biomolecular property predictions”. In: PLOS Comput. Biol. 13.7 (2017), pp. 1-27. DOI: $10.1371 /$ journal . pcbi . 1005690. URL: https://doi.org/10.1371/journal.pcbi.1005690.

[103] José Jiménez et al. "K(DEEP): Protein-Ligand Absolute Binding Affinity Prediction via 3D-Convolutional Neural Networks." In: J. Chem. Inf. Model. 58.2 (2018), pp. 287-296. ISSN: 1549-960X (Electronic). DOI: 10.1021/acs. jcim. 7bo 0650.

[104] Izhar Wallach, Michael Dzamba, and Abraham Heifets. AtomNet: A Deep Convolutional Neural Network for Bioactivity Prediction in Structure-based Drug Discovery. 2015. arXiv: 1510.02855 [CS.LG].

[105] Christof Angermueller et al. "Deep learning for computational biology". In: Mol. Syst. Biol. 12.7 (2016), p. 878. DoI: https: / / doi .org/10 . 15252/msb . 20156651. URL: https : / / www . embopress . org/doi / abs / 10 . $15252 / \mathrm{msb} .20156651$.

[106] William Jones et al. "Computational biology: deep learning". In: Emerg. Top. Life Sci. 1.3 (2017), pp. 257-274. ISSN: 2397-8554. DOI: 10.1042 /ETLS2 0160025. URL: https://doi.org/10.1042/ETLS20160025.

[107] Philippe Rocca-Serra et al. "Sharing and archiving nucleic acid structure mapping data.” In: RNA 17.7 (2011), pp. 1204-1212. ISSN: 1469-9001 (Electronic). DOI: $10.1261 /$ rna.2753211. 
[108] Pablo Cordero, Julius B Lucks, and Rhiju Das. “An RNA Mapping DataBase for curating RNA structure mapping experiments". In: Bioinformatics 28.22 (2012), pp. 3006-3008. ISSN: 1367-4803. DOI: 10.1093/bioinformatics/bts554. URL: https://doi.org/10.1093/bioinformatics/bts554.

[109] S F Altschul et al. "Basic local alignment search tool." In: J. Mol. Biol. 215.3 (1990), pp. 403-410. ISSN: 0022-2836 (Print). DOI: 10 . 1016/S0022-2836 (0 5) $80360-2$.

[110] H M Berman et al. "The Protein Data Bank.” In: Nucleic Acids Res. 28.1 (2000), pp. 235-242. ISSN: 0305-1048 (Print). DOI: 10.1093/nar/28.1.235.

[111] Kévin Darty, Alain Denise, and Yann Ponty. "VARNA: Interactive drawing and editing of the RNA secondary structure”. In: Bioinformatics 25.15 (2009), pp. 19741975. ISSN: 1367-4803. DOI: 10 . 1093 / bioinformatics/btp250. URL: https://doi.org/10.1093/bioinformatics/btp250.

[112] Tomasz Zok et al. "RNApdbee 2.0: multifunctional tool for RNA structure annotation.” In: Nucleic Acids Res. 46.W1 (2018), W30-W35. ISSN: 1362-4962 (Electronic). DOI: 10.1093/nar/gky314.

[113] Marc Parisien et al. "New metrics for comparing and assessing discrepancies between RNA 3D structures and models". eng. In: RNA 15.10 (2009), pp. 1875-1885. ISSN: 1469-9001. DOI: $10.1261 /$ rna. 1700409 . URL: https: / / pubmed. ncbi.nlm.nih.gov/19710185https://www.ncbi.nlm.nih.gov/ pmc/articles/PMC2743038/. 
[114] K He et al. "Deep Residual Learning for Image Recognition". In: 2016 IEEE Conf. Comput. Vis. Pattern Recognit. 2016, pp. 770-778. DOI: $10.1109 /$ CVPR . 2016.90 .

[115] Sergey Ioffe and Christian Szegedy. "Batch normalization: Accelerating deep network training by reducing internal covariate shift". In: arXiv Prepr. arXiv1502.03167 (2015).

[116] Vinod Nair and Geoffrey E Hinton. "Rectified linear units improve restricted boltzmann machines". In: ICML. 2010.

[117] K He et al. "Spatial Pyramid Pooling in Deep Convolutional Networks for Visual Recognition”. In: IEEE Trans. Pattern Anal. Mach. Intell. 37.9 (2015), pp. 19041916. ISSN: 1939-3539. DOI: 10 .1109/TPAMI .2015 . 2389824.

[118] Diederik P Kingma and Jimmy Ba. "Adam: A method for stochastic optimization". In: arXiv Prepr. arXiv1412.6980 (2014).

[119] Adam Paszke et al. "Automatic differentiation in PyTorch". In: (2017).

[120] The RNAcentral Consortium. "RNAcentral: a hub of information for non-coding RNA sequences". In: Nucleic Acids Res. 47.D1 (2019), pp. D221-D229. ISSN: 0305-1048. DOI: 10 . 1093 / nar / gky1034. URL: https : / / doi . org / $10.1093 /$ nar/gky1034.

[121] Jason R Stagno et al. "Heavy-atom labeling of RNA by PLOR for de novo crystallographic phasing". In: PLoS One 14.4 (2019), pp. 1-9. DOI: 10 . 1371 / journal . pone.0215555. URL: https:// doi.org/10.1371/ journal.pone. 0215555. 
[122] Krishna Choudhary, Fei Deng, and Sharon Aviran. "Comparative and integrative analysis of RNA structural profiling data: current practices and emerging questions". In: Quant. Biol. 5.1 (2017), pp. 3-24. ISSN: 2095-4697. DOI: 10.1007 / s40484-017-0093-6. URL: https: / / doi.org/10.1007/s40484$017-0093-6$

[123] David Loughrey et al. "SHAPE-Seq 2.0: systematic optimization and extension of high-throughput chemical probing of RNA secondary structure with next generation sequencing”. eng. In: Nucleic Acids Res. 42.21 (2014), e165-e165. ISSN: 1362-4962. DOI: 10.1093/nar/gku909. URL: https: / / pubmed. ncbi . nlm.nih.gov/25303992https://www.ncbi.nlm.nih.gov/pmc/ articles/PMC 4245970 /.

[124] Aleksandar Spasic et al. "Modeling RNA secondary structure folding ensembles using SHAPE mapping data”. In: Nucleic Acids Res. 46.1 (2017), pp. 314-323. ISSN: 0305-1048. DOI: 10 . $1093 /$ nar/gkx1057. URL: https : / / doi . org/10.1093/nar/gkx1057.

[125] Matthew J Smola and Kevin M Weeks. "In-cell RNA structure probing with SHAPEMaP”. In: Nat. Protoc. 13.6 (2018), pp. 1181-1195. ISSN: 1750-2799. DOI: 10 . 1038 /nprot.2018.010. URL: https://doi .org/10.1038/nprot. 2018.010.

[126] Kevin M Weeks and David M Mauger. "Exploring RNA structural codes with SHAPE chemistry”. eng. In: Acc. Chem. Res. 44.12 (2011), pp. 1280-1291. ISSN: 1520-4898. DOI: 10 . 1021 / ar200051h. URL: https : / / pubmed . ncbi . nlm.nih.gov/21615079https://www.ncbi.nlm.nih.gov/pmc/ articles/PMC3177967/. 
[127] Fei Deng et al. "Data-directed RNA secondary structure prediction using probabilistic modeling”. eng. In: RNA 22.8 (2016), pp. 1109-1119. ISSN: 1469-9001. DOI: 10 . $1261 /$ rna.055756.115. URL: https : / / pubmed.ncbi . nlm.nih.gov/27251549https://www.ncbi.nlm.nih.gov/pme/ articles/PMC4931104/.

[128] Sana Vaziri, Patrice Koehl, and Sharon Aviran. "Extracting information from RNA SHAPE data: Kalman filtering approach”. In: PLoS One 13.11 (2018), e0207029. URL: https://doi.org/10.1371/journal.pone.0207029.

[129] Buvaneswari Coimbatore Narayanan et al. "The Nucleic Acid Database: new features and capabilities". eng. In: Nucleic Acids Res. 42.Database issue (2014), pp. D114D122. ISSN: 1362-4962. DOI: 10 . 1093 / nar / gkt980. URL: https : / / pubmed.ncbi.nlm.nih.gov/24185695https://www.ncbi.nlm. nih.gov/pmc/articles/PMC3964972/.

[130] Xiang-Jun Lu, Harmen J Bussemaker, and Wilma K Olson. "DSSR: an integrated software tool for dissecting the spatial structure of RNA". In: Nucleic Acids Res. 43.21 (2015), e142-e142. ISSN: 0305-1048. DOI: 10 . 1093 / nar/gkv716. URL: https://doi.org/10.1093/nar/gkv716.

[131] Quentin Vicens et al. "Local RNA structural changes induced by crystallization are revealed by SHAPE”. eng. In: RNA 13.4 (2007), pp. 536-548. ISSN: 1355-8382. DOI: 10.1261/rna.400207. URL: https://pubmed.ncbi.nlm.nih. gov/17299128https://www.ncbi.nlm.nih.gov/pmc/articles/ PMC1831858/. 
[132] Elisa Frezza et al. "The interplay between molecular flexibility and RNA chemical probing reactivities analyzed at the nucleotide level via an extensive molecular dynamics study". In: Methods 162-163 (2019), pp. 108-127. ISSN: 10462023. DoI: https://doi org/10.1016/j.ymeth.2019.05.021. URL: http://www.sciencedirect.com/science/article/pii/ S104620231830392X.

[133] Colby D Stoddard et al. "Free state conformational sampling of the SAM-I riboswitch aptamer domain”. eng. In: Structure 18.7 (2010), pp. 787-797. ISSN: 1878-4186. DOI: 10 . 1016/j . str.2010.04 .006. URL: https : // pubmed.ncbi.nlm.nih.gov/20637415https://www.ncbi.nlm. nih.gov/pmc/articles/PMC2917978/.

[134] D.A. Case et al. AMBER 2018. San Francisco, 2018.

[135] Steve Plimpton. "Fast Parallel Algorithms for Short-Range Molecular Dynamics". In: J. Comput. Phys. 117.1 (1995), pp. 1-19. ISSN: 0021-9991. DOI: https : / / doi.org/10.1006/jcph.1995.1039. URL: http://www. sciencedirect. com/science/article/pii/s002199918571039x.

[136] William H. Press et al. "Simulated Annealing Methods". In: Numer. Recipes Art Sci. Comput. 3rd. New York: Cambridge University Press, 2007, pp. 549-554.

[137] B W Matthews. "Comparison of the predicted and observed secondary structure of T4 phage lysozyme". In: Biochim. Biophys. Acta - Protein Struct. 405.2 (1975), pp. 442-451. ISSN: 0005-2795. DOI: https: // doi .org/10 .1016/00052795 (75) 90109 -9. URL: http://www.sciencedirect.com/science/ article/pii/0005279575901099. 
[138] Alexander Serganov and Dinshaw J Patel. "Molecular recognition and function of riboswitches". In: Curr. Opin. Struct. Biol. 22.3 (2012), pp. 279-286. ISSN: 0959440X. DoI: https://doi.org/10.1016/j.sbi.2012.04.005. URL: http: //wWw. sciencedirect.com/science/article/pii/ S0959440X12000711.

[139] Kevin P Larsen et al. "Relating Structure and Dynamics in RNA Biology". In: Cold Spring Harb. Perspect. Biol. 11.7 (2019). ISSN: 1943-0264 (Electronic). DOI: 10.1101/cshperspect.a032474.

[140] Feng Ding et al. "Ab initio RNA folding by discrete molecular dynamics: from structure prediction to folding mechanisms." In: RNA 14.6 (2008), pp. 1164-1173. ISSN: 1469-9001 (Electronic). DOI: $10.1261 /$ rna. 894608.

[141] Mariusz Popenda et al. "Automated 3D structure composition for large RNAs." In: Nucleic Acids Res. 40.14 (2012), e112. ISSN: 1362-4962 (Electronic). DOI: 10 . 1093/nar/gks339.

[142] W G Noid. "Perspective: Coarse-grained models for biomolecular systems." In: J. Chem. Phys. 139.9 (2013), p. 90901. ISSN: 1089-7690 (Electronic). DOI: 10 . $1063 / 1.4818908$.

[143] Jiří Šponer et al. "Molecular Dynamics Simulations of Nucleic Acids. From Tetranucleotides to the Ribosome". In: J. Phys. Chem. Lett. 5.10 (2014), pp. 1771-1782. DOI: 10 .1021/jz500557y. URL: https : / / doi .org/10 .1021/ jz500557y. 
[144] Yi He et al. "Mean-field interactions between nucleic-acid-base dipoles can drive the formation of a double helix." In: Phys. Rev. Lett. 110.9 (2013), p. 98101. ISSN: 1079-7114 (Electronic). DOI: $10.1103 /$ PhysRevLett.110.098101.

[145] Tristan Cragnolini et al. "Coarse-Grained HiRE-RNA Model for ab Initio RNA Folding beyond Simple Molecules, Including Noncanonical and Multiple Base Pairings." In: J. Chem. Theory Comput. 11.7 (2015), pp. 3510-3522. ISSN: 15499626 (Electronic). DOI: 10.1021 /acs . jctc.5b00200.

[146] Zhen Xia et al. "Coarse-grained model for simulation of RNA three-dimensional structures." In: J. Phys. Chem. B 114.42 (2010), pp. 13497-13506. ISSN: 15205207 (Electronic). DOI: $10.1021 /$ jp104926t.

[147] Natalia A Denesyuk and D Thirumalai. "Coarse-Grained Model for Predicting RNA Folding Thermodynamics". In: J. Phys. Chem. B 117.17 (2013), pp. 49014911. ISSN: 1520-6106. DOI: 10 . $1021 /$ jp401087x. URL: https: / / doi. org/10.1021/jp401087x.

[148] Ya-Zhou Shi et al. "A coarse-grained model with implicit salt for RNAs: predicting 3D structure, stability and salt effect.” In: J. Chem. Phys. 141.10 (2014), p. 105102. ISSN: 1089-7690 (Electronic). DOI: $10.1063 / 1.4894752$.

[149] Petr Šulc et al. "A nucleotide-level coarse-grained model of RNA". In: J. Chem. Phys. 140.23 (2014), p. 235102. DOI: $10.1063 / 1.4881424$. URL: https: //doi.org/10.1063/1.4881424.

[150] Michal J Boniecki et al. "SimRNA: a coarse-grained method for RNA folding simulations and 3D structure prediction.” In: Nucleic Acids Res. 44.7 (2016), e63. ISSN: 1362-4962 (Electronic). DOI: $10.1093 /$ nar/gkv1479. 
[151] David R Bell et al. "Capturing RNA Folding Free Energy with Coarse-Grained Molecular Dynamics Simulations.” In: Sci. Rep. 7 (2017), p. 45812. ISSN: 20452322 (Electronic). DOI: $10.1038 /$ srep 45812.

[152] Jaakko J Uusitalo et al. "Martini Coarse-Grained Force Field: Extension to RNA". In: Biophys. J. 113.2 (2017), pp. 246-256. ISSN: 0006-3495. DOI: https : // doi.org/10.1016/j.bpj.2017.05 .043. uRL: http: / / www . sciencedirect.com/science/article/pii/s0006349517306124.

[153] Andrew P Latham and Bin Zhang. "Maximum Entropy Optimized Force Field for Intrinsically Disordered Proteins.” In: J. Chem. Theory Comput. 16.1 (2020), pp. 773-781. ISSN: 1549-9626 (Electronic). DOI: 10 . 1021 /acs . jctc. 9b00 932.

[154] Wouter Boomsma, Jesper Ferkinghoff-Borg, and Kresten Lindorff-Larsen. "Combining Experiments and Simulations Using the Maximum Entropy Principle”. In: PLOS Comput. Biol. 10.2 (2014), pp. 1-9. DOI: 10 . 1371 / journal . pcbi. 1003406. URL: https://doi.org/10.1371/journal.pcbi.1003406.

[155] Ariel Caticha et al. "Entropic Inference". In: AIP Conf. Proc. 1305.1 (2011). DOI: 10.1063/1.3573619. URL: http://dx.doi .org/10 .1063/1. 3573619.

[156] Y Xiong and M Sundaralingam. "Two crystal forms of helix II of Xenopus laevis 5S rRNA with a cytosine bulge.” In: RNA 6.9 (2000), pp. 1316-1324. ISSN: 13558382 (Print). DOI: $10.1017 /$ s135583820000090x.

[157] Fiona M Jucker and Arthur Pardi. "Solution Structure of the CUUG Hairpin Loop: A Novel RNA Tetraloop Motif”. In: Biochemistry 34.44 (1995), pp. 14416-14427. 
ISSN: 0006-2960. DOI: 10 . 1021 / bi00044a019. URL: https : / / pubs . acs.org/doi/abs/10.1021/bi00044a019.

[158] Xinyue Zhang et al. "Nanopore electric snapshots of an RNA tertiary folding pathway.” In: Nat. Commun. 8.1 (2017), p. 1458. ISSN: 2041-1723 (Electronic). DOI: $10.1038 / s 41467-017-01588-z$.

[159] Boxuan Simen Zhao, Ian A. Roundtree, and Chuan He. "Post-transcriptional gene regulation by mRNA modifications". In: Nat. Rev. Mol. Cell Biol. 18.1 (2016), pp. 31-42. ISSN: 14710080. DOI: 10 . $1038 / \mathrm{nrm}$. 2016.132. URL: http : //dx.doi.org/10.1038/nrm.2016.132.

[160] Tian Lin Cheng et al. "MeCP2 Suppresses Nuclear MicroRNA Processing and Dendritic Growth by Regulating the DGCR8/Drosha Complex”. In: Dev. Cell 28.5 (2014), pp. 547-560. ISSN: 18781551. DOI: $10.1016 / j$.devcel.2014.01. 032. URL: http://dx.doi.org/10.1016/j.devcel.2014.01.032.

[161] Raghu R Edupuganti et al. "N(6)-methyladenosine (m(6)A) recruits and repels proteins to regulate mRNA homeostasis.” eng. In: Nat. Struct. Mol. Biol. 24.10 (2017), pp. 870-878. ISSN: 1545-9985 (Electronic). DOI: $10.1038 / \mathrm{nsmb} .3462$.

[162] Joie Rowles et al. "FTO, RNA epigenetics and epilepsy." In: Epigenetics 7.10 (2012), pp. 1094-1097. ISSN: 1559-2308 (Electronic). DOI: 10 . $4161 / \mathrm{epi}$. 21977.

[163] Eva M Jimenez-Mateos et al. "Silencing microRNA-134 produces neuroprotective and prolonged seizure-suppressive effects.” In: Nat. Med. 18.7 (2012), pp. 10871094. ISSN: 1546-170X (Electronic). DOI: $10.1038 / \mathrm{nm} .2834$. 
[164] Jun Liu et al. "m(6)A mRNA methylation regulates AKT activity to promote the proliferation and tumorigenicity of endometrial cancer." eng. In: Nat. Cell Biol. 20.9 (2018), pp. 1074-1083. ISSN: 1476-4679 (Electronic). DOI: 10 . $1038 /$ s41556-018-0174-4.

[165] Jin Zhao Ma et al. "METTL14 suppresses the metastatic potential of hepatocellular carcinoma by modulating N6-methyladenosine-dependent primary MicroRNA processing”. In: Hepatology 65.2 (2017), pp. 529-543. ISSN: 15273350. DOI: 10 . $1002 /$ hep. 28885.

[166] Mengnuo Chen et al. "RNA N6-methyladenosine methyltransferase-like 3 promotes liver cancer progression through YTHDF2-dependent posttranscriptional silencing of SOCS2”. In: Hepatology 67.6 (2018), pp. 2254-2270. ISSN: 15273350. DOI: $10.1002 /$ hep .29683.

[167] Lianpin Wu et al. "Changes of N6-methyladenosine modulators promote breast cancer progression”. In: BMC Cancer 19.1 (2019), pp. 1-12. ISSN: 14712407. DOI: $10.1186 /$ s12885-019-5538-z.

[168] Zaira Ianniello, Alessandro Paiardini, and Alessandro Fatica. "N6-Methyladenosine (m6A): A Promising New Molecular Target in Acute Myeloid Leukemia”. In: Front. Oncol. 9 (2019), p. 251. ISSN: 2234-943X. DOI: 10.3389 / fonc. 2019. 00251. URL: https://www.frontiersin.org/article/10.3389/ fonc.2019.00251.

[169] Lucas Willmann et al. "Exometabolom analysis of breast cancer cell lines: Metabolic signature.” In: Sci. Rep. 5 (2015), p. 13374. ISSN: 2045-2322 (Electronic). DOI: $10.1038 / \operatorname{srep} 13374$. 
[170] Yan P. Yu et al. "Novel fusion transcripts associate with progressive prostate cancer”. In: Am. J. Pathol. 184.10 (2014), pp. 2840-2849. ISSN: 15252191. DOI: 10 . 1016/j.ajpath.2014.06.025.

[171] Shun Sakuraba, Kiyoshi Asai, and Tomoshi Kameda. "Predicting RNA Duplex Dimerization Free-Energy Changes upon Mutations Using Molecular Dynamics Simulations”. In: J. Phys. Chem. Lett. 6.21 (2015), pp. 4348-4351. DOI: 10 . 1021/acs.jpclett.5b01984. URL: https://doi.org/10.1021/ acs.jpclett.5b01984.

[172] S Nishida et al. "Estimating Energy Parameters for RNA Secondary Structure Predictions Using Both Experimental and Computational Data". In: IEEE/ACM Trans. Comput. Biol. Bioinforma. 16.5 (2019), pp. 1645-1655. ISSN: 1557-9964. DOI: $10.1109 / \mathrm{TCBB} .2018 .2813388$.

[173] X Chen, R Kierzek, and D H Turner. "Stability and structure of RNA duplexes containing isoguanosine and isocytidine." In: J. Am. Chem. Soc. 123.7 (2001), pp. 1267-1274. ISSN: 0002-7863 (Print). DOI: 10.1021 / ja002623i.

[174] Daniel J Wright et al. "Nearest neighbor parameters for inosine $\mathrm{x}$ uridine pairs in RNA duplexes.” In: Biochemistry 46.15 (2007), pp. 4625-4634. ISSN: 0006-2960 (Print). DOI: $10.1021 /$ bi0616910.

[175] Caroline Roost et al. "Structure and thermodynamics of N6-methyladenosine in RNA: A spring-loaded base modification”. In: J. Am. Chem. Soc. 137 (2015), pp. 2107-2115. ISSN: 15205126. DOI: $10.1021 /$ ja513080v. 
[176] Fang-Chieh Chieh Chou et al. "Blind tests of RNA nearest-neighbor energy prediction”. eng. In: Proc. Natl. Acad. Sci. U. S. A. 113.30 (2016), pp. 8430-8435. ISSN: 1091-6490 (Electronic). DOI: 10 .1073/pnas.1523335113.

[177] Daniel J Wright, Christopher R Force, and Brent M Znosko. "Stability of RNA duplexes containing inosine-cytosine pairs.” In: Nucleic Acids Res. 46.22 (2018), pp. 12099-12108. ISSN: 1362-4962 (Electronic). DOI: 10 . 1093/nar/gky907.

[178] Melissa C Hopfinger, Charles C Kirkpatrick, and Brent M Znosko. "Predictions and analyses of RNA nearest neighbor parameters for modified nucleotides". In: $\mathrm{Nu}$ cleic Acids Res. 48.16 (2020), pp. 8901-8913. ISSN: 0305-1048. DOI: $10.1093 /$ nar/gkaa654. URL: https://doi.org/10.1093/nar/gkaa654.

[179] Olke C. Uhlenbeck. “Tetra loops and RNA folding”. In: Nature 346 (1990), pp. 613614. DOI: $10.1038 / 346613 a 0$.

[180] G. Varani. "Exceptionally Stable Nucleic Acid Hairpins". In: Annu. Rev. Biophys. Biomol. Struct. 24 (1995), pp. 379-404. ISSN: 10568700. DOI: 10 . 1146 / annurev . biophys.24.1.379.

[181] Nan Jie Deng and Piotr Cieplak. "Free energy profile of RNA hairpins: A molecular dynamics simulation study”. In: Biophys. J. 98.4 (2010), pp. 627-636. ISSN: 15420086. DOI: $10.1016 / j . b p j .2009 .10 .040$. URL: http://dx.doi. org/10.1016/j.bpj.2009.10.040.

[182] Daniel Seeliger and Bert L de Groot. "Protein Thermostability Calculations Using Alchemical Free Energy Simulations”. In: Biophys. J. 98.10 (2010), pp. 23092316. ISSN: 0006-3495. DOI: https : // doi .org/10 . 1016/j . bpj . 
2010.01.051. URL: http://WWw.sciencedirect.com/science/ article/pii/s000634951000216X.

[183] Piero Procacci. "I. Dissociation free energies of drugreceptor systems via nonequilibrium alchemical simulations: a theoretical framework". In: Phys. Chem. Chem. Phys. 18.22 (2016), pp. 14991-15004. DOI: 10.1039 / C5CP 05519 A. URL: http://dx.doi.org/10.1039/C5CP05519A.

[184] Jiř́ Šponer et al. "RNA Structural Dynamics As Captured by Molecular Simulations: A Comprehensive Overview". In: Chem. Rev. 118.8 (2018), pp. 4177-4338. ISSN: 0009-2665. DOI: 10 . 1021 / acs . chemrev . 7b00427. URL: https: //doi.org/10.1021/acs.chemrev.7b00427.

[185] Tai-Sung Lee et al. "Alchemical Binding Free Energy Calculations in AMBER20: Advances and Best Practices for Drug Discovery”. In: J. Chem. Inf. Model. (2020). ISSN: 1549-9596. DOI: 10 .1021/acs . jcim. 0c00613. URL: https : / / doi.org/10.1021/acs.jcim.0c00613.

[186] Yaozong Li and Kwangho Nam. "Repulsive Soft-Core Potentials for Efficient Alchemical Free Energy Calculations". In: J. Chem. Theory Comput. 16.8 (2020), pp. 4776-4789. ISSN: 1549-9618. DOI: 10 .1021/acs . jctc. 0 c0 0163 . URL: https://doi.org/10.1021/acs.jctc.0c00163.

[187] Yoshiaki Tanida and Azuma Matsuura. "Alchemical free energy calculations via metadynamics: Application to the theophylline-RNA aptamer complex". In: J. Comput. Chem. 41.20 (2020), pp. 1804-1819. DOI: 10.1002 / jCC. 26221. URL: https: / / onlinelibrary.wiley.com/doi/abs/10.1002/jcc. 26221. 
[188] Vojtěch Mlýnský and Giovanni Bussi. "Exploring RNA structure and dynamics through enhanced sampling simulations". In: Curr. Opin. Struct. Biol. 49 (2018), pp. 63-71. ISSN: 0959-440X. DOI: https:// doi org/10.1016/j.sbi. 2018.01.004. URL: http://WWW.sciencedirect.com/science/ article/pii/s0959440X17301008.

[189] Xiaolin Cheng et al. "Modified replica exchange simulation methods for local structure refinement." In: J. Phys. Chem. B 109.16 (2005), pp. 8220-8230. ISSN: 15206106 (Print). DOI: $10.1021 /$ jp0 45437 y.

[190] Nan-Jie Deng and Piotr Cieplak. "Molecular Dynamics and Free Energy Study of the Conformational Equilibria in the UUUU RNA Hairpin”. In: J. Chem. Theory Comput. 3.4 (2007), pp. 1435-1450. ISSN: 1549-9618. DOI: 10.1021 / ct 6003388. URL: https://doi.org/10.1021/ct6003388.

[191] Alessandra Villa, Elisabeth Widjajakusuma, and Gerhard Stock. "Molecular Dynamics Simulation of the Structure, Dynamics, and Thermostability of the RNA Hairpins uCACGg and cUUCGg”. In: J. Phys. Chem. B 112.1 (2008), pp. 134142. ISSN: 1520-6106. DOI: 10 . $1021 /$ jp0764337. URL: https: / / doi . org/10.1021/jp0764337.

[192] Angel E Garcia and Dietmar Paschek. "Simulation of the Pressure and Temperature Folding/Unfolding Equilibrium of a Small RNA Hairpin". In: J. Am. Chem. Soc. 130.3 (2008), pp. 815-817. ISSN: 0002-7863. DOI: 10.1021 / ja 074191 i. URL: https://doi.org/10.1021/ja074191i.

[193] Yufen Zhang, Xian Zhao, and Yuguang Mu. "Conformational Transition Map of an RNA GCAA Tetraloop Explored by Replica-Exchange Molecular Dynamics 
Simulation". In: J. Chem. Theory Comput. 5.4 (2009), pp. 1146-1154. ISSN: 15499618. DoI: 10 .1021/ct8004276. URL: https: / / doi .org/10.1021/ ct 8004276 .

[194] Allison J DePaul et al. "Equilibrium conformational dynamics in an RNA tetraloop from massively parallel molecular dynamics". In: Nucleic Acids Res. 38.14 (2010), pp. 4856-4867. ISSN: 0305-1048. DOI: 10 .1093/nar/gkq134. URL: https : //doi.org/10.1093/nar/gkq134.

[195] Guanghong Zuo et al. "Folding of a Small RNA Hairpin Based on Simulation with Replica Exchange Molecular Dynamics”. In: J. Phys. Chem. B 114.17 (2010), pp. 5835-5839. ISSN: 1520-6106. DOI: 10 . 1021/ jp904573r. URL: https: //doi.org/10.1021/jp904573r.

[196] Petra Kührová et al. "Computer Folding of RNA Tetraloops? Are We There Yet?" In: J. Chem. Theory Comput. 9.4 (2013), pp. 2115-2125. ISSN: 1549-9618. DOI: 10.1021/ct301086z. URL: https://doi.org/10.1021/ct301086z.

[197] Sandro Bottaro, Alejandro Gil-Ley, and Giovanni Bussi. "RNA folding pathways in stop motion." eng. In: Nucleic Acids Res. 44.12 (2016), pp. 5883-5891. ISSN: 1362-4962 (Electronic). DOI: $10.1093 /$ nar/gkw239.

[198] Petra Kührová et al. "Computer Folding of RNA Tetraloops: Identification of Key Force Field Deficiencies". In: J. Chem. Theory Comput. 12.9 (2016), pp. 4534 4548. ISSN: 1549-9618. DOI: 10 .1021/acs . jctc.6b00300. URL: https: //doi.org/10.1021/acs.jctc.6b00300.

[199] Jacob C Miner, Alan A Chen, and Angel E García. "Free-energy landscape of a hyperstable RNA tetraloop". In: Proc. Natl. Acad. Sci. 113.24 (2016), pp. 6665- 
6670. ISSN: 0027-8424. DOI: $10.1073 /$ pnas . 1603154113. URL: https: //wWw.pnas.org/content/113/24/6665.

[200] Sandro Bottaro et al. "Conformational ensembles of RNA oligonucleotides from integrating NMR and molecular simulations”. In: Sci. Adv. 4.5 (2018). DOI: 10 . 1126 / sciadv • aar8521. URL: https : / / advances . sciencemag • org/content/4/5/eaar8521.

[201] Andrea Cesari et al. "Fitting Corrections to an RNA Force Field Using Experimental Data”. In: J. Chem. Theory Comput. 15.6 (2019), pp. 3425-3431. ISSN: 1549-9618. DOI: 10.1021/acs . jetc.9b00206. URL: https : / / doi . org/10.1021/acs.jctc.9b00206.

[202] D. Jeremy Williams and Kathleen B. Hall. "Experimental and computational studies of the G[UUCG]C RNA tetraloop”. In: J. Mol. Biol. 297.5 (2000), pp. 10451061. ISSN: 00222836. DOI: $10.1006 /$ jmbi.2000.3623.

[203] Susana Carregal-Romero et al. "MicroRNA Nanotherapeutics for Lung Targeting. Insights into Pulmonary Hypertension.” In: Int. J. Mol. Sci. 21.9 (2020). ISSN: 1422-0067 (Electronic). DOI: 10.3390 / i jms 21093253.

[204] Thomas J. Macke and David A. Case. "Modeling Unusual Nucleic Acid Structures”. In: Mol. Model. Nucleic Acids. Ed. by N. B. Leontes and J. SantaLucia, Jr. Washington, DC, 1998. Chap. 24, pp. 379-393. DOI: 10 .1021/bk-1998$0682 . \operatorname{ch} 024$.

[205] Eric F Pettersen et al. "UCSF Chimera-a visualization system for exploratory research and analysis." In: J. Comput. Chem. 25.13 (2004), pp. 1605-1612. ISSN: 
0192-8651. DOI: $10.1002 /$ jcc.20084. URL: http://www.ncbi.nlm. nih.gov/pubmed/15264254.

[206] Agastya P Bhati et al. "Uncertainty Quantification in Alchemical Free Energy Methods.” In: J. Chem. Theory Comput. 14.6 (2018), pp. 2867-2880. ISSN: 15499626 (Electronic). DOI: 10.1021 /acs . jctc. 7 b01143.

[207] Thomas Steinbrecher, David L. Mobley, and David A. Case. "Nonlinear scaling schemes for Lennard-Jones interactions in free energy calculations". In: J. Chem. Phys. 127.21 (2007). ISSN: 00219606. DOI: $10.1063 / 1.2799191$.

[208] Michael R Shirts and David L Mobley. "An Introduction to Best Practices in Free Energy Calculations". In: Biomol. Simulations Methods Protoc. Ed. by Luca Monticelli and Emppu Salonen. Totowa, NJ: Humana Press, 2013, pp. 271-311. ISBN: 978-1-62703-017-5. DOI: 10 . $1007 / 978-1-62703-017-5$ _11. URL: https://doi.org/10.1007/978-1-62703-017-5_11.

[209] Pavel V Klimovich, Michael R Shirts, and David L Mobley. "Guidelines for the analysis of free energy calculations." In: J. Comput. Aided. Mol. Des. 29.5 (2015), pp. 397-411. ISSN: 1573-4951 (Electronic). DOI: 10 . 1007 / s10822-015$9840-9$.

[210] Daniel R Roe and Thomas E Cheatham. "PTRAJ and CPPTRAJ: Software for Processing and Analysis of Molecular Dynamics Trajectory Data." In: J. Chem. Theory Comput. 9.7 (2013), pp. 3084-3095. ISSN: 1549-9618. DOI: $10.1021 /$ ct 400341 . URL: http: //www.ncbi.nlm.nih.gov/pubmed/26583988. 
[211] Michael R Shirts and John D Chodera. "Statistically optimal analysis of samples from multiple equilibrium states.” In: J. Chem. Phys. 129.12 (2008), p. 124105. ISSN: 1089-7690 (Electronic). DOI: $10.1063 / 1.2978177$.

[212] David H. Mathews et al. "Incorporating chemical modification constraints into a dynamic programming algorithm for prediction of RNA secondary structure". In: Proc. Natl. Acad. Sci. 101.19 (2004), pp. 7287-7292. ISSN: 0027-8424. DOI: 10 . 1073/pnas . 0401799101 arXiv: NIHMS150003. URL: https: / / www . pnas.org/content/101/19/7287.

[213] Timothy J Giese and Darrin M York. "A GPU-Accelerated Parameter Interpolation Thermodynamic Integration Free Energy Method." In: J. Chem. Theory Comput. 14.3 (2018), pp. 1564-1582. ISSN: 1549-9626 (Electronic). DOI: 10 . 1021 / acs . jetc. 7 bo 01175 .

[214] Xibing He et al. "Fast, Accurate, and Reliable Protocols for Routine Calculations of ProteinLigand Binding Affinities in Drug Design Projects Using AMBER GPUTI with ff14SB/GAFF". In: ACS Omega 5.9 (2020), pp. 4611-4619. ISSN: 24701343. DOI: 10 . 1021/acsomega .9b04233. URL: https: / / doi .org/ 10.1021 /acsomega.9b04233.

[215] Zhuoyun Zhuang, Luc Jaeger, and Joan Emma Shea. "Probing the structural hierarchy and energy landscape of an RNA T-loop hairpin”. In: Nucleic Acids Res. 35.20 (2007), pp. 6995-7002. ISSN: 03051048. DOI: 10 .1093/nar/gkm719.

[216] Tom Darden, Darrin York, and Lee Pedersen. "Particle mesh Ewald: An N $\log (\mathrm{N})$ method for Ewald sums in large systems”. In: J. Chem. Phys. 98.12 (1993), pp. 1008910092. ISSN: 00219606. DOI: $10.1063 / 1.464397$. 
[217] Edina Rosta and Nicolae-Viorel Buchete. "Thermostat artifacts in replica exchange molecular dynamics simulations". In: J. Chem. Theory Comput. 5.5 (2009), pp. $1393-$ 1399. ISSN: 1549-9618. DOI: $10.1021 /$ ct $800557 \mathrm{~h}$. Thermostat.

[218] Alexandra Patriksson and David Van Der Spoel. "A temperature predictor for parallel tempering simulations". In: Phys. Chem. Chem. Phys. 10 (2008), pp. 20732077. ISSN: 14639076. DOI: 10 . 1039/b716554d.

[219] Martin Ester et al. "Density-Based Clustering Methods". In: Proc. Second Int. Conf. Knowl. Discov. Data Min. 1996, pp. 226-231. ISBN: 9780444527011. DOI: 10 . 1016/B978-044452701-1.00067-3.

[220] T Caliński and J Harabasz. "A dendrite method for cluster analysis". In: Commun. Stat. 3.1 (1974), pp. 1-27. DOI: 10 . $1080 / 03610927408827101$. URL: https://www.tandfonline.com/doi/abs/10.1080/03610927408827101.

[221] D L Davies and D W Bouldin. "A Cluster Separation Measure”. In: IEEE Trans. Pattern Anal. Mach. Intell. PAMI-1.2 (1979), pp. 224-227. ISSN: 0162-8828. DOI: 10.1109/TPAMI.1979.4766909.

[222] Robert W. Zwanzig. "High-Temperature Equation of State by a Perturbation Method. I. Nonpolar Gases”. In: J. Chem. Phys. 22.8 (1954), pp. 1420-1426. ISSN: 10897690. DOI: $10.1063 / 1.1740409$. 


\section{VITA}

Travis Hurst was born on September 15, 1990, in Cape Girardeau, Missouri. He began research with Dr. Shi-Jie Chen in 2012 and earned B.S. degrees in Biochemistry and Physics at the University of Missouri-Columbia in 2013 and 2014. After leaving the Navy in 2016, he went on to pursue his PhD degree in Computational Biological Physics from the University of Missouri-Columbia. He earned his M.S. degree in Physics at University of Missouri-Columbia in 2019, advised by Dr. Shi-Jie Chen.

Travis Hurst married Alyson Hurst on April 13, 2019 in Fayetteville, Texas. 